\title{
Acyl-ghrelin mediated lipid retention and inflammation within in vitro and ex vivo adipose depots
}

\section{Churm, Rachel}

How to cite:

Churm, Rachel (2018) Acyl-ghrelin mediated lipid retention and inflammation within in vitro and ex vivo adipose depots. Doctoral thesis, Swansea University.

http://cronfa.swan.ac.uk/Record/cronfa40925

Use policy:

This item is brought to you by Swansea University. Any person downloading material is agreeing to abide by the terms of the repository licence: copies of full text items may be used or reproduced in any format or medium, without prior permission for personal research or study, educational or non-commercial purposes only. The copyright for any work remains with the original author unless otherwise specified. The full-text must not be sold in any format or medium without the formal permission of the copyright holder. Permission for multiple reproductions should be obtained from the original author.

Authors are personally responsible for adhering to copyright and publisher restrictions when uploading content to the repository.

Please link to the metadata record in the Swansea University repository, Cronfa (link given in the citation reference above.)

http://www.swansea.ac.uk/library/researchsupport/ris-support/ 
Acyl-ghrelin mediated lipid retention and inflammation within in vitro and ex vivo adipose depots

\section{Rachel Churm}

Submitted to Swansea University in the fulfilment of the requirements for the Degree of Doctor of Philosophy

Diabetes Research Group

Medical School

Swansea University

2018 


\section{Summary}

The 28 amino acid hormone, ghrelin, has been found to have various effects on metabolism. This thesis focuses on the pathways integrated into ghrelin's effect within adipocytes and adipose tissue depots of those with and without Type 2 diabetes. To determine whether acyl-ghrelin plays a role in mediating the metabolic state in an in vitro and ex vivo setting this thesis investigates cellular mechanisms via the analysis of: lipid staining, lipid retention gene expression pathway, inflammatory marker levels and determination of oxidative burden. This project confirms and translates previous murine model findings that establishes a mediatory role for acyl-ghrelin within lipid retention. Furthermore, this mechanism is influenced and magnified within the presence of hyperglycaemia, indicating that the impact of glucose metabolism on acyl-ghrelin and lipid homeostasis may result in the deterioration of dyslipidaemia. In addition to novel findings relating to lipid retention, results indicate that acyl-ghrelin also impacts the inflammatory state. Acyl-ghrelin exposure resulted in a marked decrease in pro-inflammatory marker IL6, and ghrelin mRNA expression was associated with an increase in IL-10 and total antioxidant status. The promotion of the inflammatory state in the presence of acyl-ghrelin may yield novel therapeutic avenues for acyl-ghrelin combination treatment in the amelioration of the low-grade inflammation present within Type 2 diabetes. 


\section{Declaration and statements}

This work has not previously been accepted in substance for any degree and is not being concurrently submitted in candidature for any degree.

Signed

Date

\section{STATEMENT 1}

This thesis is the result of my own investigations, except where otherwise stated. Where correction services have been used, the extent and nature of the correction is clearly marked in a footnote(s).

Other sources are acknowledged by footnotes giving explicit references. A bibliography is appended.

Signed

Date

\section{STATEMENT 2}

I hereby give consent for my thesis, if accepted, to be available for photocopying and for inter-library loan, and for the title and summary to be made available to outside organisations.

Signed

Date 


\section{Table of Contents}

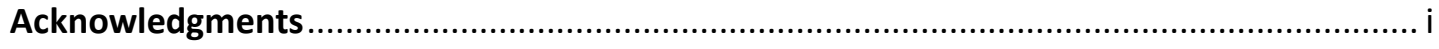

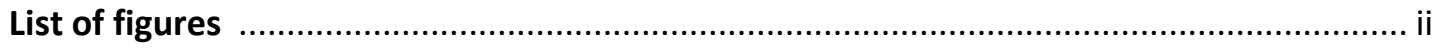

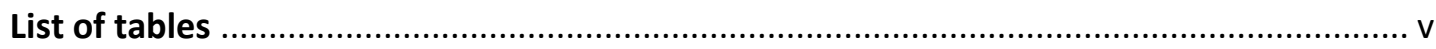

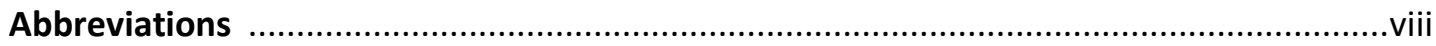

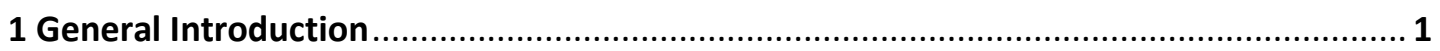

1.1 Diabetes Mellitus ......................................................................................... 2

1.1.1 Glucose and insulin homeostasis .......................................................... 2

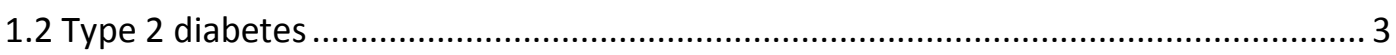

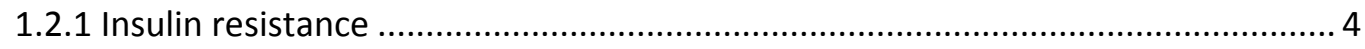

1.2.2 $\beta$-cell dysfunction and destruction ................................................... 5

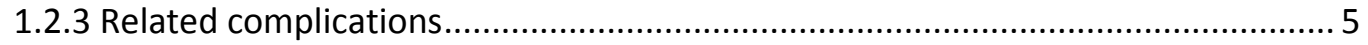

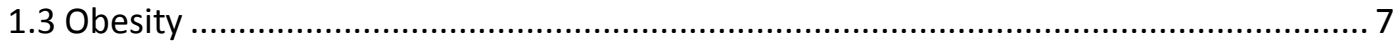

1.3.1 Environmental and genetic causes of obesity .............................................. 9

1.3.2 Comorbidities linked to obesity .......................................................... 9

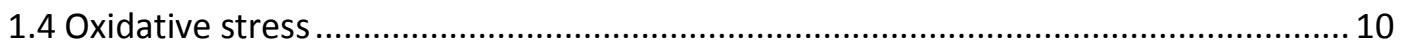

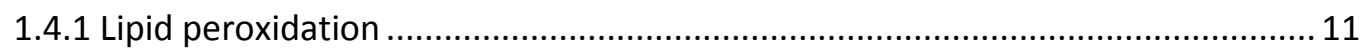

1.4.2 Inflammation and ROS production ...................................................... 12

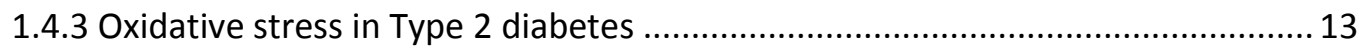

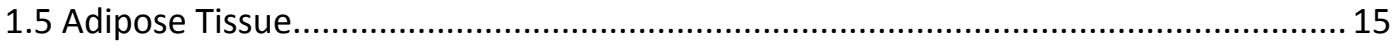

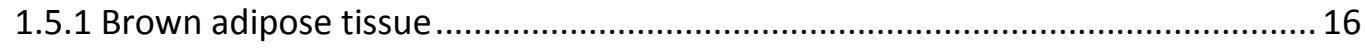

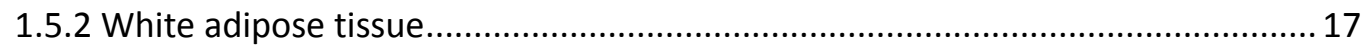

1.5.3 Beige adipose tissue....................................................................... 18

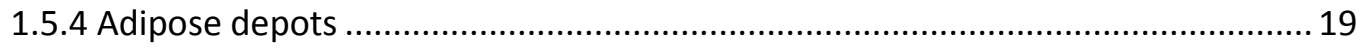

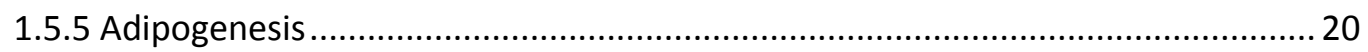

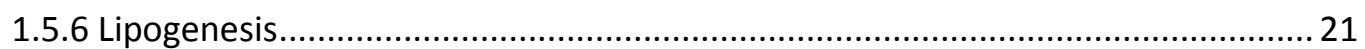

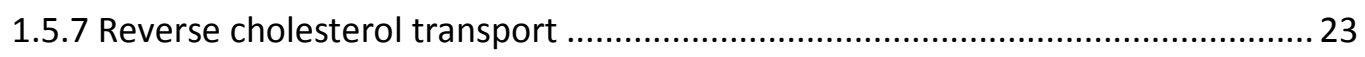

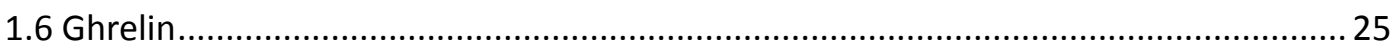

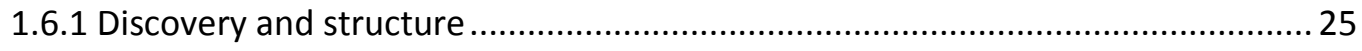

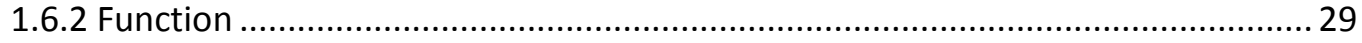

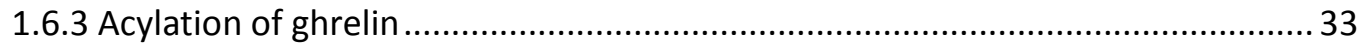

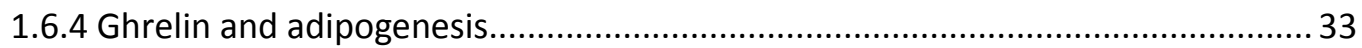

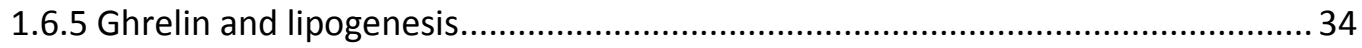




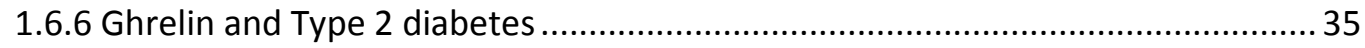

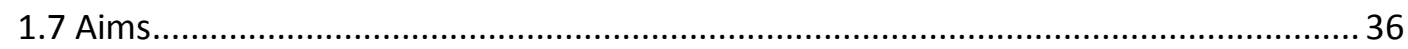

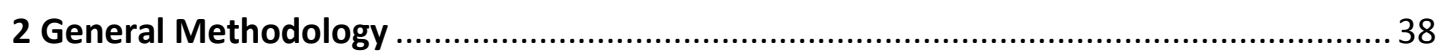

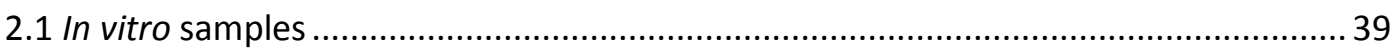

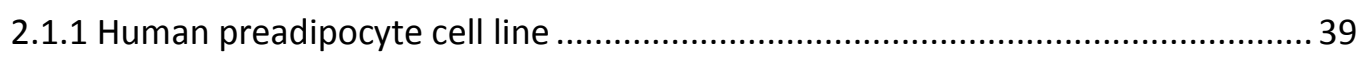

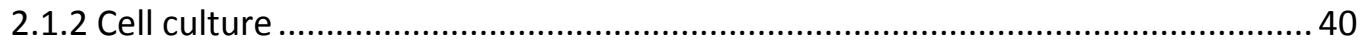

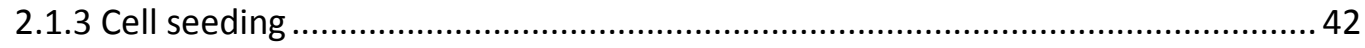

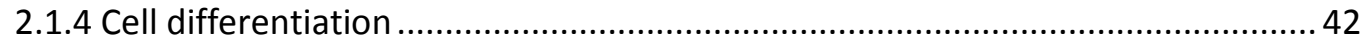

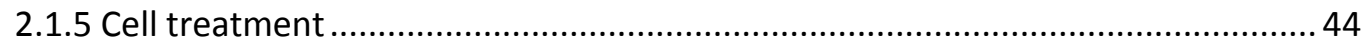

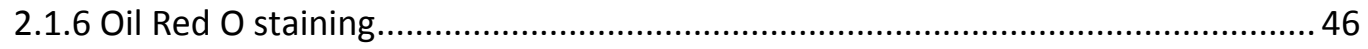

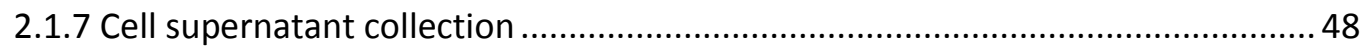

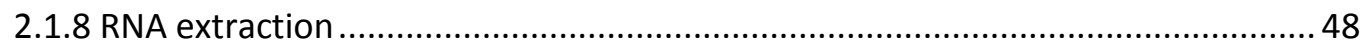

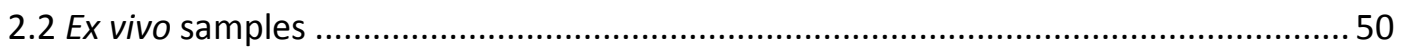

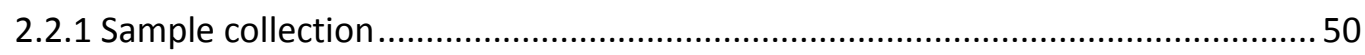

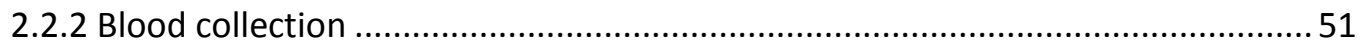

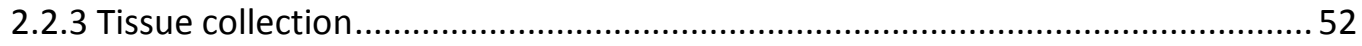

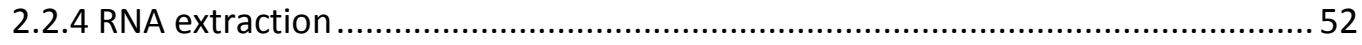

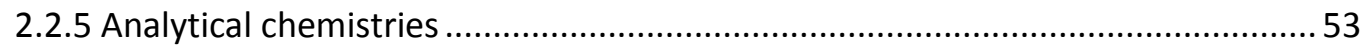

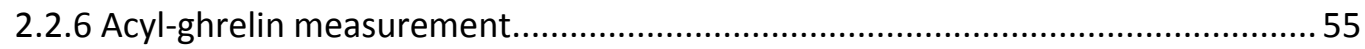

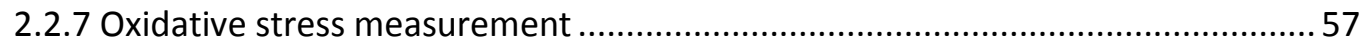

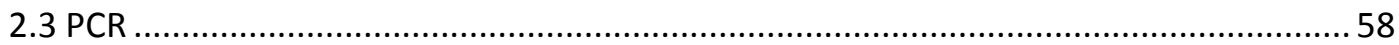

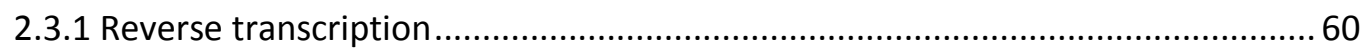

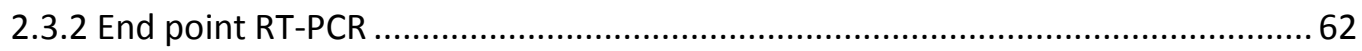

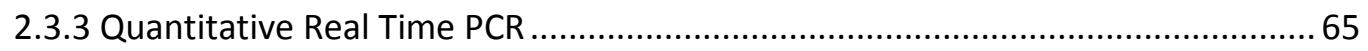

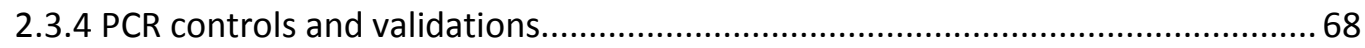

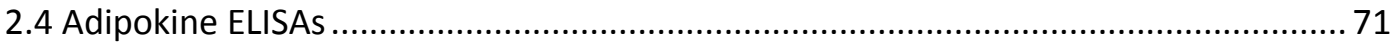

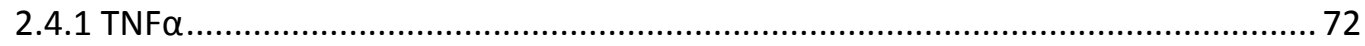

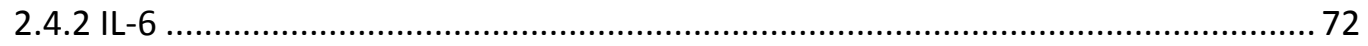

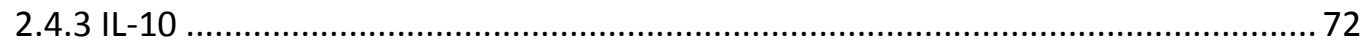

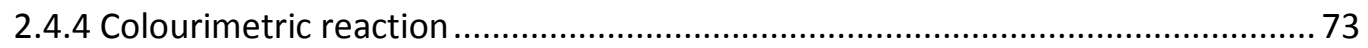

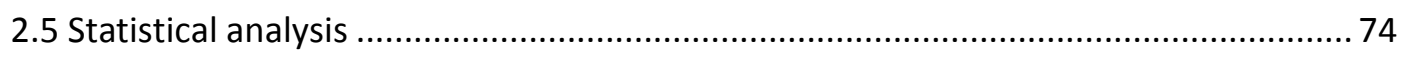

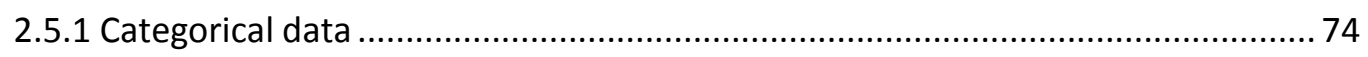

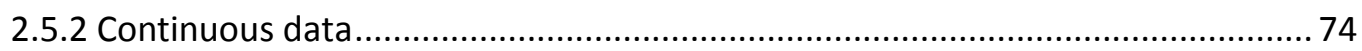


3 Effect of acyl-ghrelin in vitro on mature adipocytes in hyper and normoglycaemic

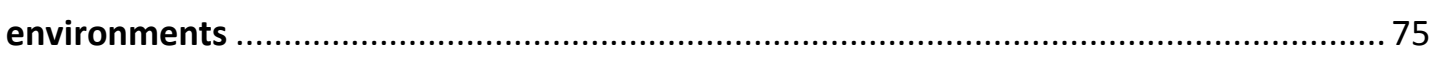

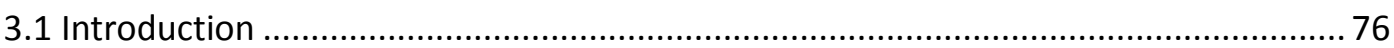

3.1.1 Lipid droplet storage in mature adipocytes....................................................... 76

3.1.2 Hypertrophy induced inflammation and Type 2 diabetes................................... 77

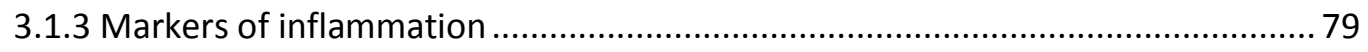

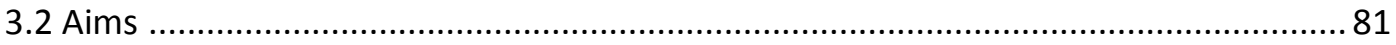

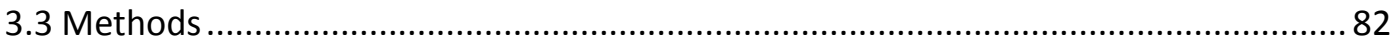

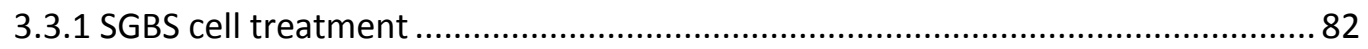

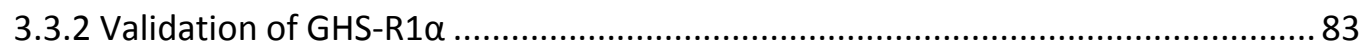

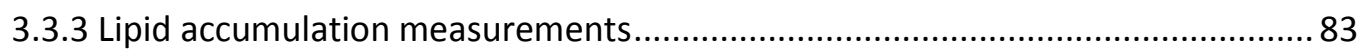

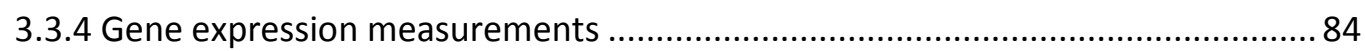

3.3.5 Inflammatory marker measurements.............................................................. 85

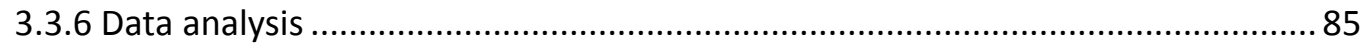

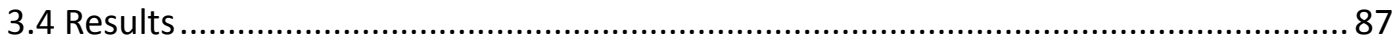

3.4.1 Acyl-ghrelin treated human mature adipocytes in a normoglycaemic environment

3.4.2 Acyl-ghrelin treated human mature adipocytes in a hyperglycaemic environment

3.4.3 Comparison of glycaemic environment on acyl-ghrelin treated human mature

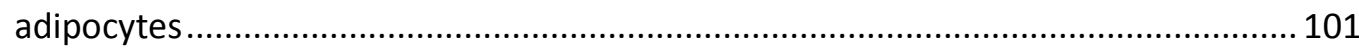

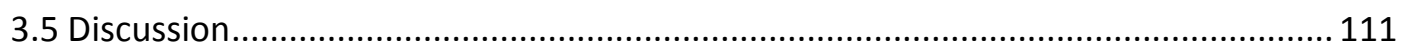

3.5.1 Acyl-ghrelin mediated lipid retention ................................................................ 111

3.5.2 Acyl-ghrelin mediated inflammatory response ................................................ 113

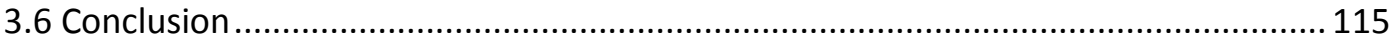

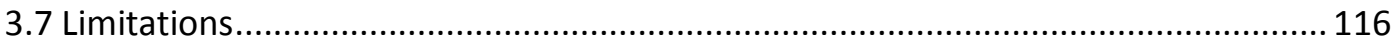

4 Exploration of acyl-ghrelin associated lipid retention within ex vivo human visceral

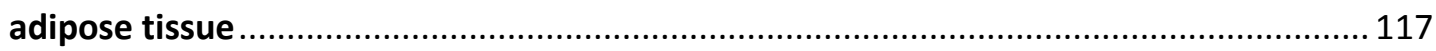

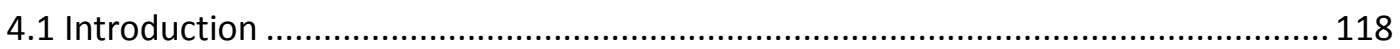

4.1.1 Adipose tissue, inflammation and oxidative stress .......................................... 120

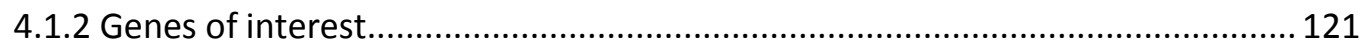

4.2 Aims

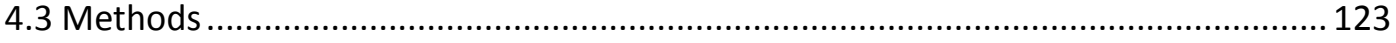

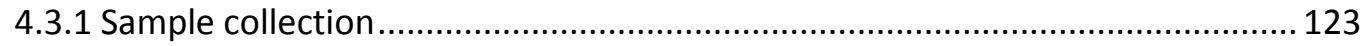

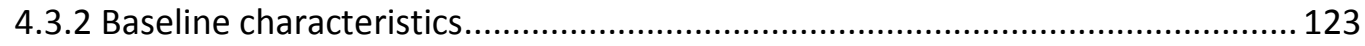


4.3.4 Gene expression analysis .................................................................. 124

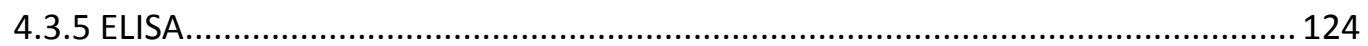

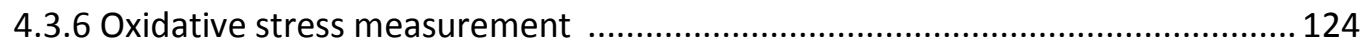

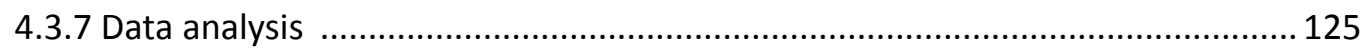

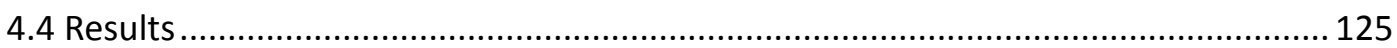

4.4.1 Total sample cohort ................................................................. 125

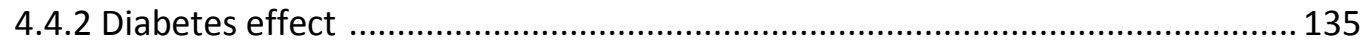

4.4.3 Obesity effect................................................................................. 143

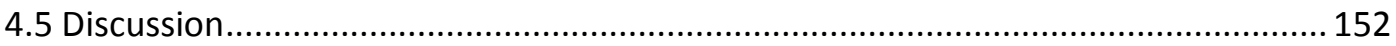

4.5.1 Circulating acyl-ghrelin levels ............................................................ 152

4.5.2 Acyl-ghrelin mediated lipid retention ...................................................... 154

4.5.2 Acyl-ghrelin and the inflammatory state ............................................... 156

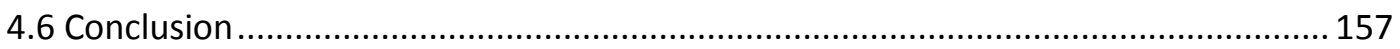

4.7 Limitations........................................................................................... 158

5 The effect of combining metformin and acyl-ghrelin exposure on lipid retention and

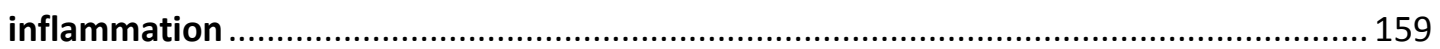

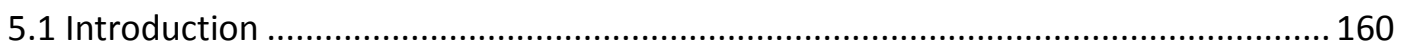

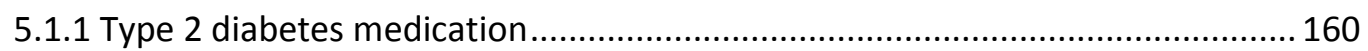

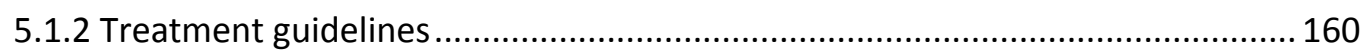

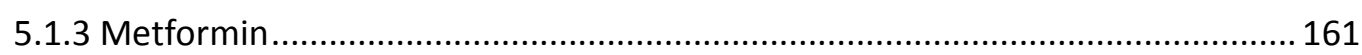

5.1.4 Proposed mechanism for metformin action......................................... 162

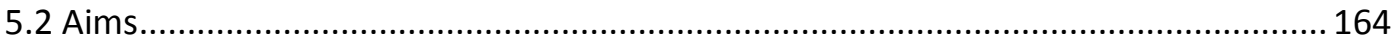

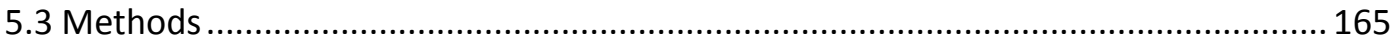

5.3.1 SGBS cell treatment ........................................................................ 165

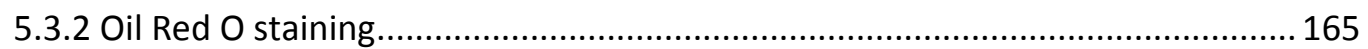

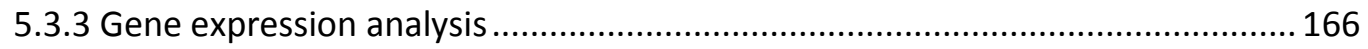

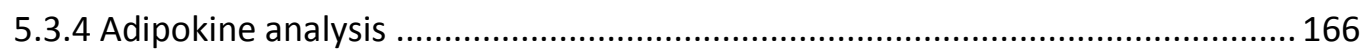

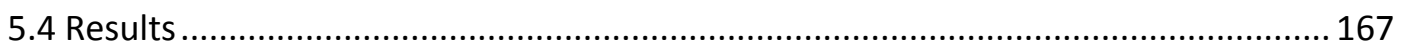

5.4.1 Effect of metformin on human mature adipocytes ..................................... 167

5.4.2 Effect of metformin in combination with acyl-ghrelin exposure on human mature

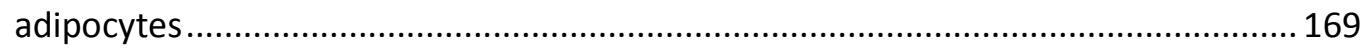

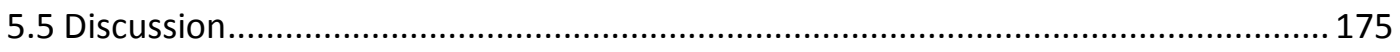

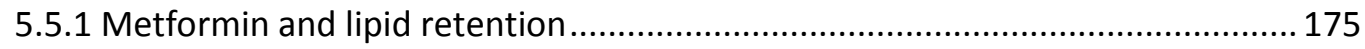


5.5.2 Metformin and inflammation 177

5.6 Conclusion 178

5.7 Limitations. 178

6 General Discussion 179

Appendix 1 Ethical approval \& patient information sheet

Appendix 2 Primer sequences and programmes

Appendix 3 Primer optimisation and validation

Appendix 4 Real Time PCR validation (Raw data)

Appendix 5 Raw $\Delta \Delta$ Ct data for SGBS cells

Appendix 6 Raw $\Delta \Delta C t$ data for hVAT

Appendix 7 Raw $\Delta \Delta C t$ data for metformin treatment of SGBS cells

Appendix 8 Publications

Appendix 9 Conference abstract list

Bibliography 


\section{Acknowledgements}

I would like to express great gratitude to a vast number of people who have made the construction of this thesis possible.

Firstly, I would like to thank Dr Sarah Prior for not only giving me the opportunity to take on the project but to support me throughout the project as a supervisor and as a true friend.

I am also extremely grateful to my secondary supervisors, Dr Jeffrey Davies and Professor Jeffrey Stephens for supporting me through the last 3 years and for also providing endless feedback when required. Their feedback allowed for the production of this thesis and has given me the ability to speak in public without giggling.

To the bariatric team at Morriston Hospital; Mr Jon Barry, Mr Scott Caplin and Mrs Nia Jenkins, for introducing me to participants and enabling sample collection, without which I would not have a whole fourth chapter!

Furthermore, I would like to thank the team at the Diabetes Research Unit for always being there for advice when needed; especially Professor Stephen Luzio and Dr Gareth Dunseath, for giving me the opportunity to work within the Unit and be introduced to this amazing opportunity.

Finally, a shout out to my friends and family that have endured me over time and provided me with the love and support needed to ensure this project and thesis was completed. 


\section{Figure Contents}

\begin{tabular}{|c|c|c|}
\hline $\begin{array}{l}\text { Figure } \\
\text { Number }\end{array}$ & Title & $\begin{array}{l}\text { Page } \\
\text { Number }\end{array}$ \\
\hline Figure 1.1 & BMI graph & 8 \\
\hline Figure 1.2 & Overview of oxidative stress within Type 2 diabetes & 14 \\
\hline Figure 1.3 & $\begin{array}{l}\text { Different cell lineages that produce brown, beige and white } \\
\text { adipocytes }\end{array}$ & 16 \\
\hline Figure 1.4 & An example of adipokines secreted by white adipose tissue & 18 \\
\hline Figure 1.5 & Pathway of fatty acid production & 23 \\
\hline Figure 1.6 & Reverse cholesterol transport within humans & 24 \\
\hline Figure 1.7 & $\begin{array}{l}\text { Amino acid sequence of ghrelin and the acetylation of the } \\
\text { serine } 3 \text { residue }\end{array}$ & 26 \\
\hline Figure 1.8 & $\begin{array}{l}\text { Ghrelin structure with a cross indicating the loss of an amino } \\
\text { acid residue to produce des-GLN 14-ghrelin via mRNA splicing }\end{array}$ & 27 \\
\hline Figure 1.9 & Ghrelin synthesis and maturation within a stomach cell & 28 \\
\hline Figure 1.10 & Cognitive and peripheral effect of ghrelin & 31 \\
\hline Figure 2.1 & Media components for 14 day differentiation period & 44 \\
\hline Figure 2.2 & $\begin{array}{l}\text { Plate layouts for cell treatments, replicated for high and low } \\
\text { glucose concentrations }\end{array}$ & 46 \\
\hline Figure 2.3. & $\begin{array}{l}\text { Mature SGBS cells imaged at magnification x50 before and after } \\
\text { Oil red O staining and haematoxylin counterstain }\end{array}$ & 48 \\
\hline Figure 2.4 & Key steps involved in a sandwich ELISA & 56 \\
\hline Figure 2.5 & $\begin{array}{l}\text { Polymerase Chain Reaction (PCR), three main stages and } \\
\text { description }\end{array}$ & 59 \\
\hline Figure 2.6. & Plate layout for adipokine ELISAs & 71 \\
\hline Figure 3.1 & $\begin{array}{l}\text { Overview of the downward effect of hypertrophic adipocytes in } \\
\text { obesity }\end{array}$ & 78 \\
\hline Figure 3.2 & $12 \%$ polyacrylamide gel showing PCR products for GHSR1 $\alpha$ & 83 \\
\hline Figure 3.3 & Plate layout for adipokine ELISA for each experimental repeat & 86 \\
\hline $\begin{array}{l}\text { Figure } \\
\text { 3.4.A-D }\end{array}$ & $\begin{array}{l}\text { Representative Oil red O imaging of mature SGBS cells in } 5.5 \\
\text { mM (normoglycaemia) glucose at x } 50 \text { magnification }\end{array}$ & 88 \\
\hline
\end{tabular}




\begin{tabular}{|c|c|c|}
\hline Figure 3.5 & $\begin{array}{l}\text { Scatter graph between cell diameter }(\mu \mathrm{m}) \text { and lipid area }\left(\mu \mathrm{m}^{2}\right) \\
\text { of all cell treatments in normoglycaemic environment }\end{array}$ & 89 \\
\hline $\begin{array}{l}\text { Figure } 3.6 \\
\text { A-C }\end{array}$ & $\begin{array}{l}\text { Relative fold change values for gene expression data in acyl- } \\
\text { ghrelin treated cells in normoglycaemic environment in } \\
\text { comparison to vehicle at both time points ( } 17 \text { and } 34 \text { hours) }\end{array}$ & 90 \\
\hline $\begin{array}{l}\text { Figure } 3.7 \\
\text { A-D }\end{array}$ & $\begin{array}{l}\text { Oil red O imaging of mature SGBS cells in } 25 \mathrm{mM} \\
\text { (hyperglycaemia) glucose at x50 magnification }\end{array}$ & 95 \\
\hline Figure 3.8 & $\begin{array}{l}\text { Scatter graph between cell diameter }(\mu \mathrm{m}) \text { and lipid area }\left(\mu \mathrm{m}^{2}\right) \\
\text { of all cell treatments in a hyperglycaemic environment }\end{array}$ & 96 \\
\hline $\begin{array}{l}\text { Figure } 3.9 \\
\text { A-C }\end{array}$ & $\begin{array}{l}\text { Relative fold change values for gene expression data in acyl- } \\
\text { ghrelin treated cells in hyperglycaemic environment in } \\
\text { comparison to vehicle at both time points ( } 17 \text { and } 34 \text { hours) }\end{array}$ & 97 \\
\hline Figure 3.10 & $\begin{array}{l}\text { Cellular lipid area for acyl-ghrelin and vehicle treated cells } \\
\text { within both } 5.5 \mathrm{mM} \text { (normoglycaemia) and } 25 \mathrm{mM} \\
\text { (hyperglycaemia) glucose for } 17 \text { hours }\end{array}$ & 102 \\
\hline Figure 3.11 & $\begin{array}{l}\text { Cellular lipid area for acyl-ghrelin and vehicle treated cells } \\
\text { within both } 5.5 \mathrm{mM} \text { (normoglycaemia) and } 25 \mathrm{mM} \\
\text { (hyperglycaemia) glucose for } 34 \text { hours }\end{array}$ & 102 \\
\hline $\begin{array}{l}\text { Figure } 3.12 \\
\text { A-C }\end{array}$ & $\begin{array}{l}\text { Relative fold change values for gene expression data in vehicle } \\
\text { treated cells in hyperglycaemic environment in comparison to } \\
\text { normoglycaemic environment at both time points (17 and } 34 \\
\text { hours) }\end{array}$ & 105 \\
\hline $\begin{array}{l}\text { Figure } 3.13 \\
\text { A-C }\end{array}$ & $\begin{array}{l}\text { Relative fold change values for gene expression data in acyl- } \\
\text { ghrelin treated cells in hyperglycaemic environment in } \\
\text { comparison to normoglycaemic environment at both time } \\
\text { points ( } 17 \text { and } 34 \text { hours) }\end{array}$ & 106 \\
\hline Figure 4.1 & Visceral fat depots in the human body & 119 \\
\hline Figure 4.2 & Box plot of fasting acyl-ghrelin levels within the three cohorts & 127 \\
\hline $\begin{array}{l}\text { Figure } 4.3 \\
\text { A-B }\end{array}$ & $\begin{array}{l}\text { Scatter graphs between acyl-ghrelin and glucose, and acyl- } \\
\text { ghrelin and body weight }\end{array}$ & 127 \\
\hline $\begin{array}{l}\text { Figure } 4.4 \\
\text { A-B }\end{array}$ & $\begin{array}{l}\text { Scatter graphs between acyl-ghrelin and total cholesterol, and } \\
\text { acyl-ghrelin and LDL }\end{array}$ & 131 \\
\hline Figure 4.5 & Bar graph of mean TAOS (\%) within the three cohorts & 134 \\
\hline Figure 4.6 & Box plot of fasting acyl-ghrelin levels for diabetes effect & 137 \\
\hline
\end{tabular}




\begin{tabular}{|c|c|c|}
\hline Figure 4.7 & Bar graph of mean TAOS (\%) for diabetes effect & 141 \\
\hline $\begin{array}{l}\text { Figure } 4.8 \\
\text { A-B }\end{array}$ & $\begin{array}{l}\text { Scatter graphs between acyl-ghrelin levels and BMI, and acyl- } \\
\text { ghrelin and body weight }\end{array}$ & 145 \\
\hline $\begin{array}{l}\text { Figure } 4.9 \\
\text { A-B }\end{array}$ & $\begin{array}{l}\text { Scatter graphs between GHRL expression and total cholesterol, } \\
\text { and acyl-ghrelin and LDL }\end{array}$ & 148 \\
\hline Figure 4.10 & Bar graph of mean TAOS (\%) for obesity effect & 150 \\
\hline Figure 5.1 & $\begin{array}{l}\text { Oil red O imaging of mature SGBS cells in } 25 \mathrm{mM} \\
\text { (hyperglycaemia) glucose and metformin treatment at x50 } \\
\text { magnification }\end{array}$ & 167 \\
\hline Figure 5.2 & $\begin{array}{l}\text { Relative fold change values for gene expression data in } \\
\text { metformin treated cells in hyperglycaemic environment in } \\
\text { comparison to control at } 17 \text { hours }\end{array}$ & 168 \\
\hline Figure 5.3 & $\begin{array}{l}\text { Box plot of IL- } 6 \text { and IL-10 levels within metformin and control } \\
\text { cells in hyperglycaemic environment }\end{array}$ & 169 \\
\hline Figure 5.4 & $\begin{array}{l}\text { Box plot of intracellular lipid area within vehicle, acyl-ghrelin, } \\
\text { metformin and metformin/acyl-ghrelin treated cells in } \\
\text { hyperglycaemic environment }\end{array}$ & 170 \\
\hline Figure 5.5 & $\begin{array}{l}\text { Box plot of IL-6 levels within vehicle, acyl-ghrelin, metformin } \\
\text { and metformin/acyl-ghrelin treated cells in hyperglycaemic } \\
\text { environment }\end{array}$ & 174 \\
\hline Figure 6.1 & $\begin{array}{l}\text { Gene expression profile over time (17 and } 34 \text { hours) of cells in } \\
\text { normoglycaemic environment treated with either acyl-ghrelin } \\
\text { or vehicle }\end{array}$ & 181 \\
\hline Figure 6.2 & $\begin{array}{l}\text { Gene expression profile over time (17 and } 34 \text { hours) of cells in } \\
\text { hyperglycaemic environment treated with either acyl-ghrelin or } \\
\text { vehicle }\end{array}$ & 182 \\
\hline
\end{tabular}


Table Contents

\begin{tabular}{|c|c|c|}
\hline $\begin{array}{l}\text { Table } \\
\text { Number }\end{array}$ & Title & $\begin{array}{l}\text { Page } \\
\text { Number }\end{array}$ \\
\hline Table 1.1 & BMI classifications & 8 \\
\hline Table 2.1 & Media A components & 39 \\
\hline Table 2.2 & Differentiation media B components & 42 \\
\hline Table 2.3 & Differentiation media C components & 42 \\
\hline Table 2.4 & $\begin{array}{l}\text { Reverse transcription primers used within cDNA synthesis } \\
\text { for each specific primer set }\end{array}$ & 61 \\
\hline Table 2.5 & $\begin{array}{l}\text { Intra- and inter-variations for Real Time PCR for all primer } \\
\text { sets }\end{array}$ & 70 \\
\hline Table 3.1 & $\begin{array}{l}\text { Lipid area and cellular diameter for acyl-ghrelin and vehicle } \\
\text { treated cells in normoglycaemic environment }\end{array}$ & 88 \\
\hline Table 3.2 & $\begin{array}{l}\text { IL-6 levels for acyl-ghrelin and vehicle treated cells in } \\
\text { normoglycaemic environment }\end{array}$ & 91 \\
\hline Table 3.3 & $\begin{array}{l}\text { TNF } \alpha \text { levels for acyl-ghrelin and vehicle treated cells in } \\
\text { normoglycaemic environment }\end{array}$ & 92 \\
\hline Table 3.4 & $\begin{array}{l}\text { IL-10 levels for acyl-ghrelin and vehicle treated cells in } \\
\text { normoglycaemic environment }\end{array}$ & 93 \\
\hline Table 3.5 & $\begin{array}{l}\text { Lipid area and cellular diameter for acyl-ghrelin and vehicle } \\
\text { treated cells in hyperglycaemic environment }\end{array}$ & 95 \\
\hline Table 3.6 & $\begin{array}{l}\text { IL-6 levels for acyl-ghrelin and vehicle treated cells in } \\
\text { hyperglycaemic environment }\end{array}$ & 98 \\
\hline Table 3.7 & $\begin{array}{l}\text { TNFa levels for acyl-ghrelin and vehicle treated cells in } \\
\text { hyperglycaemic environment }\end{array}$ & 99 \\
\hline Table 3.8 & $\begin{array}{l}\text { IL-10 levels for acyl-ghrelin and vehicle treated cells in } \\
\text { hyperglycaemic environment }\end{array}$ & 100 \\
\hline Table 3.9 & $\begin{array}{l}\text { Comparison of IL- } 6 \text { levels in different environments at } 17 \\
\text { hours }\end{array}$ & 107 \\
\hline Table 3.10 & $\begin{array}{l}\text { Comparison of IL- } 6 \text { levels in different environments at } 34 \\
\text { hours }\end{array}$ & 107 \\
\hline
\end{tabular}




\begin{tabular}{|c|c|c|}
\hline Table 3.11 & $\begin{array}{l}\text { Comparison of TNF } \alpha \text { levels in different environments at } 17 \\
\text { hours }\end{array}$ & 108 \\
\hline Table 3.12 & $\begin{array}{l}\text { Comparison of TNF } \alpha \text { levels in different environments at } 34 \\
\text { hours }\end{array}$ & 109 \\
\hline Table 3.13 & $\begin{array}{l}\text { Comparison of IL-10 levels in different environments at } 17 \\
\text { hours }\end{array}$ & 110 \\
\hline Table 3.14 & $\begin{array}{l}\text { Comparison of IL-10 levels in different environments at } 34 \\
\text { hours }\end{array}$ & 110 \\
\hline Table 4.1 & Baseline data for total cohort & 126 \\
\hline Table 4.2 & $\begin{array}{l}\text { Relative fold change values for ghrelin axis gene expression } \\
\text { data for total cohort }\end{array}$ & 129 \\
\hline Table 4.3 & $\begin{array}{l}\text { Correlation data for gene expression and plasma acyl-ghrelin } \\
\text { for total cohort }\end{array}$ & 129 \\
\hline Table 4.4 & Lipid profiles for total cohort & 130 \\
\hline Table 4.5 & $\begin{array}{l}\text { Relative fold change values for lipid retention gene } \\
\text { expression data for total cohort }\end{array}$ & 132 \\
\hline Table 4.6 & Cytokine levels for total cohort & 133 \\
\hline Table 4.7 & $\begin{array}{l}\text { Correlation data for gene expression, plasma acyl-ghrelin } \\
\text { levels and inflammatory markers for total cohort }\end{array}$ & 135 \\
\hline Table 4.8 & Baseline data for diabetes effect cohort & 136 \\
\hline Table 4.9 & $\begin{array}{l}\text { Relative fold change values for ghrelin axis gene expression } \\
\text { data for diabetes effect cohort }\end{array}$ & 138 \\
\hline Table 4.10 & $\begin{array}{l}\text { Correlation data for gene expression and plasma acyl-ghrelin } \\
\text { for diabetes effect cohort }\end{array}$ & 138 \\
\hline Table 4.11 & Lipid profiles for diabetes effect cohort & 139 \\
\hline Table 4.12 & Relative fold change values for lipid retention gene & 140 \\
\hline
\end{tabular}




\begin{tabular}{|c|c|c|}
\hline & expression data for diabetes effect cohort & \\
\hline Table 4.13 & Cytokine levels for diabetes effect cohort & 141 \\
\hline Table 4.14 & $\begin{array}{l}\text { Correlation data for gene expression, plasma acyl-ghrelin } \\
\text { levels and inflammatory markers for diabetes effect cohort }\end{array}$ & 142 \\
\hline Table 4.15 & Baseline data for obesity effect cohort & 144 \\
\hline Table 4.16 & $\begin{array}{l}\text { Relative fold change values for ghrelin axis gene expression } \\
\text { data for obesity effect cohort }\end{array}$ & 145 \\
\hline Table 4.17 & $\begin{array}{l}\text { Correlation data for gene expression and plasma acyl-ghrelin } \\
\text { for obesity effect cohort }\end{array}$ & 146 \\
\hline Table 4.18 & Lipid profiles for obesity effect cohort & 147 \\
\hline Table 4.19 & $\begin{array}{l}\text { Relative fold change values for lipid retention gene } \\
\text { expression data for obesity effect cohort }\end{array}$ & 148 \\
\hline Table 4.20 & Cytokine levels for obesity effect cohort & 149 \\
\hline Table 4.21 & $\begin{array}{l}\text { Correlation data for gene expression, plasma acyl-ghrelin } \\
\text { levels and inflammatory markers for obesity effect cohort }\end{array}$ & 151 \\
\hline Table 4.22 & $\begin{array}{l}\text { Effects of acyl-ghrelin treatment on adipogenesis in both } \\
\text { local and global adipose regions }\end{array}$ & 155 \\
\hline Table 5.1 & $\begin{array}{l}\text { Relative fold change values for gene expression data in } \\
\text { vehicle, acyl-ghrelin and metformin/acyl-ghrelin treated cells } \\
\text { in hyperglycaemic environment at } 17 \text { hours }\end{array}$ & 172 \\
\hline Table 5.2 & $\begin{array}{l}\text { IL-10 levels for all treated cells in hyperglycaemic } \\
\text { environment }\end{array}$ & 173 \\
\hline
\end{tabular}




\section{Abbreviations}

\begin{tabular}{|c|c|}
\hline Abbreviation & Description \\
\hline$A B$ & Antibody \\
\hline ABCA1 & ATP-binding cassette sub-family A member 1 \\
\hline ABCG1 & ATP-binding cassette sub-family $\mathrm{G}$ member 1 \\
\hline ABTS $^{+}$ & 2,2-azino-bis-3-ethylbensthiazoline-6-sulfonic acid radical \\
\hline AESBF & 4-(2-Aminoethyl)-benzenesulfonyl fluoreide \\
\hline AGE & Advanced glycation end-product \\
\hline AMPK & AMP-activated protein kinase \\
\hline ANOVA & Analysis of variance \\
\hline APT1 & Acyl-protein thioesterase 1 \\
\hline ARC & Arcuate nucleus \\
\hline ATM & Adipose tissue macrophages \\
\hline BAT & Brown adipose tissue \\
\hline BMI & Body mass index \\
\hline BP & Base pair \\
\hline CAMP & Cyclic adenosine monophosphate \\
\hline cDNA & Complementary DNA \\
\hline CHD & Coronary heart disease \\
\hline CNS & Central nervous system \\
\hline Ct & Threshold cycle \\
\hline CVD & Cardiovascular disease \\
\hline DM & Diabetes mellitus \\
\hline DNL & De novo lipogenesis \\
\hline
\end{tabular}




\begin{tabular}{|c|c|}
\hline DPP-4 & Dipeptidyl peptidase-4 \\
\hline EI-MS & Election ionisation mass spectroscopy \\
\hline ELISA & Enzyme linked immunosorbent assay \\
\hline ERK & Extracellular signal-regulated kinase \\
\hline FCS & Foetal calf serum \\
\hline FDG & [18F]-2-Fluoro-D-2-deoxy-D-glucose \\
\hline FFA & Free fatty acid \\
\hline GH & Growth hormone \\
\hline GHSR-1 & Growth hormone secretagogue receptor 1 \\
\hline GIP & Gastric inhibitory polypeptide \\
\hline GLN & Glutamine \\
\hline GLP-1 & Glucagon like peptide \\
\hline GLUT4 & Glucose transporter-4 \\
\hline GOAT & Ghrelin-O-acyltransferase \\
\hline GSH & Glutathione \\
\hline HbA1c & Relative percentage of glycosylated haemoglobin in whole blood \\
\hline HDL & High density lipoprotein \\
\hline HOMA-IR & Homeostatic model assessment of insulin resistance \\
\hline HPLC & High performance liquid chromatography \\
\hline HRP & Horseradish peroxidase \\
\hline hVAT & Human visceral adipose tissue \\
\hline IBMX & 3-isobutyl-1-methylxanthine \\
\hline IGF-1 & Insulin growth factor 1 \\
\hline IL & Interleukin \\
\hline IQR & Interquartile range \\
\hline
\end{tabular}




\begin{tabular}{|c|c|}
\hline IRS-1 & Insulin receptor substrate-1 \\
\hline JNK & c-Jun N-Terminal Kinase \\
\hline LDL & Low density lipoprotein \\
\hline LPS & Lipopolysaccharide \\
\hline LXR & Liver $\mathrm{X}$ receptor \\
\hline LYPLA1 & Lysophospholipase I \\
\hline M1 & Classically activated macrophages \\
\hline M2 & Alternatively activated macrophages \\
\hline MAPK & Mitogen-activated protein kinase \\
\hline mBOAT4 & Membrane bound O-acyltransferase domain containing 4 \\
\hline MCFA & Medium chain fatty acid \\
\hline MODY & Maturity onset diabetes of the young \\
\hline mTORC1 & Mammalian target of rapamycin complex 1 \\
\hline NADPH & Nicotinamide adenine dinucleotide phosphate \\
\hline NFKB & Nuclear factor kappa-light-chain-enhancer of activated B cells \\
\hline NHS & National Health Service \\
\hline NICE & National Institute for Health and Care Excellence \\
\hline NPY & Neuropeptide $Y$ \\
\hline NRTC & No reverse transcription control \\
\hline NTC & No template control \\
\hline PBS & Phosphate buffered saline \\
\hline $\mathrm{PC1} / 3$ & Prohormone convertase $1 / 3$ \\
\hline PCR & Polymerase chain reaction \\
\hline PET & Positron emission tomography \\
\hline PPAR & Peroxisome proliferator-activated receptor \\
\hline
\end{tabular}




\begin{tabular}{|c|c|}
\hline PVD & Peripheral vascular disease \\
\hline PWS & Prader Willis syndrome \\
\hline qRealTime PCR & Quantitative Real Time polymerase chain reaction \\
\hline ROS & Reactive oxygen species \\
\hline RPM & Rotations per minute \\
\hline RT-PCR & Reverse transcription polymerase chain reaction \\
\hline SD & Standard deviation \\
\hline SGBS & Simpson-Golabi-Behmel syndrome \\
\hline SGLT2 & Sodium-glucose co-transporter-2 \\
\hline SIRT1 & Sirtuin 1 \\
\hline SOD & Superoxide dismutase \\
\hline SREBP-1a/c & Sterol regulatory element binding protein- $1 \mathrm{a} / \mathrm{c}$ \\
\hline SREPF1 & Sterol regulatory element binding factor 1 \\
\hline T1D & Type 1 diabetes mellitus \\
\hline T2D & Type 2 diabetes mellitus \\
\hline TAOS & Total antioxidant status \\
\hline TMB & 3, 3', 5, 5'-tetramethylbenzidine. \\
\hline TNF $\alpha$ & Tumor necrosis factor alpha \\
\hline UCP1 & Uncoupling protein 1 \\
\hline UV & Ultraviolet \\
\hline VAT & Visceral adipose tissue \\
\hline WAT & White adipose tissue \\
\hline
\end{tabular}


Chapter 1

General Introduction 


\subsection{Diabetes Mellitus}

Diabetes Mellitus (DM) is a multifactorial complex carbohydrate metabolic disorder. Individuals that have DM normally exhibit abnormal blood glucose levels due to insufficient insulin levels or activity. DM is separated into four main groups:

1) Type 1 diabetes mellitus (T1D) - accounts for $5-10 \%$ of all diabetes diagnosis (American Diabetes Association, 2016) and is due to cellular mediated autoimmune destruction of the pancreatic $\beta$-cells.

2) Type 2 diabetes mellitus (T2D) - accounts for $90-95 \%$ of all diabetes and this form encompasses individuals with insulin resistance and relative insulin deficiency.

3) Gestational diabetes - diabetes diagnosed within the second or third trimester of pregnancy and is not clearly overt diabetes.

4) Other specific types of diabetes - condition caused by other factors i.e. monogenic factors, such as neonatal diabetes and maturity onset diabetes of the young (MODY) (American Diabetes Association, 2016).

\subsubsection{Glucose and insulin homeostasis}

Serum glucose levels, in a healthy state, remain relatively steady throughout the course of the day. To allow for this plateau, glucoregulatory factors, derived from many tissues, are responsible for glucose homeostasis (Plum et al., 2006). The circulation of glucose is dependent upon the rate in which it enters the circulation and the balance of its removal. Circulating glucose is derived from various processes 
i.e. intestinal absorption and hepatic processes involving glycogen breakdown (glycogenolysis) and glucose formation (gluconeogenesis) (Aronoff et al., 2004, Plum et al., 2006). The pancreas plays a fundamental role in glucose homeostasis. In a fasting state, pancreatic $\alpha$-cells secrete glucagon at an increased rate, whilst $\beta$ cells secrete insulin in the presence of elevated glucose levels. This allows for a decrease in glucose levels by promoting glucose uptake within peripheral tissues (predominantly adipose, skeletal muscle and liver), promoting liver glycogenesis and inhibiting glucagon secretion from $\alpha$-cells. In addition to glucose, insulin stimulus can be in the form of increased plasma concentrations of certain incretins i.e. amino acids (arginine) and gut hormones (gastric inhibitory polypeptide (GIP) and glucagon-like protein (GLP)-1) (Aronoff et al., 2004). However in DM, this highly regulated balance of circulating glucose is hindered due to an insulin response deficiency (Aronoff et al., 2004).

\subsection{Type 2 diabetes}

Research has shown that patients with T2D are highly likely to be overweight or obese. The LEADER 5 study demonstrated within nearly 10,000 individuals with T2D there was a high prevalence of overweight (29\%) and obesity (62\%) (Masmiquel et al., 2016). An excess of abdominal fat is thought to result in the release of proinflammatory cytokines leading to insulin resistance. Obesity is a global epidemic which will result in an increase in the number of people with T2D rising from 415 million in 2015 to 642 million in 2040 globally (International Diabetes Federation, 2016). T2D is a progressive condition linked to both environmental and genetic 
factors that prevent the maintenance of normal glucose homeostasis due to insulin resistance and $\beta$-cell dysfunction (Popa and Mota, 2013). T2D is the combination of three main aetiologies: insulin resistance, $\beta$-cell dysfunction and $\beta$-cell destruction.

\subsubsection{Insulin resistance}

Insulin producing $\beta$-cells are situated in the Islet of Langherans in the pancreas, and predominantly respond to blood glucose levels by the synthesis and secretion of insulin. Insulin is initially synthesised as a single chain precursor, preproinsulin. A signal peptide is removed when it enters the endoplasmic reticulum generating proinsulin; and finally the C-peptide molecule is excised to produce the mature form of insulin. Secreted along with insulin is a neuroendocrine hormone, amylin, complementing insulin's action. Obese individuals classically display the onset of insulin resistance which is counteracted by increased insulin secretion and $\beta$-cell mass, avoiding the onset of T2D (Popa and Mota, 2013). Insulin resistance develops due to the body's inability to utilise insulin efficiency. Individuals at risk of T2D display an initial state of insulin resistance that is often counteracted by pancreatic $\beta$-cell overproduction of insulin. The early stages of insulin resistance can occur up to 15 years before T2D onset. Ultimately an increase in hepatic glucose production becomes too great for insulin reserves to cope with, resulting in an increased need of insulin secretion. The combination of insufficient insulin levels and insulin resistance leads to the development of hyperglycaemia. The molecular mechanism(s) that underpin insulin resistance have not been clearly defined. 
However, it is associated with body mass and inversely correlated with physical activity (Tangvarasittichai, 2015).

\subsection{2 $\beta$-cell dysfunction and destruction}

In genetically predisposed individuals, $\beta$-cells can develop a change in insulin secretion threshold, with a reduction in insulin gene expression and a decrease in $\beta$ cell mass. It is a combination of insulin resistance and genetic predisposition that results in an increase in glucose and free fatty acid levels. Chronic exposure of $\beta$ cells to high glucose levels can result in apoptosis via the increase of pro-apoptotic gene expression (Popa and Mota, 2013), as well as glucolipotoxicity causing $\beta$-cell dysfunction and destruction via the production of islet amyloid polypeptides (Kahn et al., 1999). In a high glucose environment, there is an increase of toxic amyloid fibrils, resulting in amyloid fibril accumulations and ultimately $\beta$-cell dysfunction and destruction (Popa and Mota, 2013, Kahn et al., 1999).

\subsubsection{Related complications}

T2D is a heterogeneous disorder that results in a vast array of related complications. T2D can result in insulin resistance, inflammation and dyslipidaemia, which when combined add to the risk of further related complications. Complications can be grouped as either; macrovascular (e.g. cardiovascular disease (CVD)); or microvascular (e.g. diabetic nephropathy, retinopathy and neuropathy). 
The level of glucose that can result in endothelial dysfunction is relatively low, therefore, patients that are yet to be diagnosed with T2D are still at an increased risk of developing macrovascular and microvascular complications (American Diabetes Association, 2016).

Previous findings report a linear correlation between exposure to high glucose levels and the risks of vascular complications and mortality (Zoungas et al., 2012). The association results in approximately $80 \%$ of individuals with T2D being at risk of death from associated cardiovascular complications (Martín-Timón et al., 2014). CVD encompasses coronary heart disease (CHD), stroke and peripheral vascular disease (PVD), all of which are the result of atherosclerosis and inflammation within arteries. This increased risk results from hyperglycaemia triggering dyslipidaemia and increasing oxidative stress (Stephens et al., 2009), activating numerous biochemical pathways linked with diabetes-associated vascular disease (Johansen et al., 2005).

Microvascular complications develop as a result of increased glucose levels having a detrimental effect on small blood vessels within the eye (retinopathy), kidney (nephropathy) and nerves (neuropathy). Diabetic retinopathy is one of the leading causes of visual impairment in the Western world (Marques-Neves, 2015). The hyperglycaemic environment associated with T2D results in changes in retina cell metabolism and alterations in biochemical balance that lead to retinal cell death (Madsen-Bouterse and Kowluru, 2008). Diabetic nephropathy effects approximately $20-40 \%$ of people with T2D (Gross et al., 2005), with vascular damage to capillaries within the glomeruli, altering the efficacy of waste filtration, 
resulting in mircroaluminuria and eventually proteinuria. Diabetic neuropathy arises as a result of peripheral nerve dysfunction caused by an increase in reactive oxygen species and the production of advanced glycation end products (AGEs) (Fowler, 2008). The disease can affect approximately up to $32 \%$ of people with T1D and T2D and it results in numbness, tingling or pain and isolated or multiple nerve palsies (Stratton et al., 2000, Fedele et al., 1997).

As with all microvascular and macrovascular complications, the risk of developing the disease is increased with prolonged exposure to hyperglycaemia, with this also influencing severity. Furthermore, as well as the length of hyperglycaemic exposure, some individuals may hold a genetic predisposition to develop the disorder.

\subsection{Obesity}

Obesity is often defined as an excess of body fat that adversely affects health. As a multifactorial disease, it has many causes but predominantly a chronic excess in energy intake compared to energy expenditure. Obesity is often classified by body mass index (BMI) (Table 1.1, Figure 1.1). BMI is the same for both genders and adults of all ages, however it does not take into consideration an individual's configuration, therefore leading to a rise in the use of alternatives such as body fat percentage indicators.

The prevalence of obesity has more than doubled since 1980; with predictions over the next 20 years for numbers to increase by $73 \%$ to approximately 
26 million people (World Health Organization, 2016). This increase in the prevalence of obesity is seen in both developed and developing countries; with the rate of childhood obesity reported to result in a global increase to 70 million overweight or obese children by 2025 (World Health Organization, 2016).

\begin{tabular}{|l|l|}
\hline BMI $\left(\mathrm{kg} / \mathrm{m}^{2}\right)$ & WHO class \\
\hline$<\mathbf{1 8 . 5}$ & Underweight \\
\hline $\mathbf{1 8 . 5}-\mathbf{2 4 . 9}$ & Healthy \\
\hline $\mathbf{2 5 - 2 9 . 9}$ & Overweight (Pre-obese) \\
\hline $\mathbf{3 0 - 3 4 . 9}$ & Obese class I \\
\hline $\mathbf{3 5 - 3 9 . 9}$ & Obese class II \\
\hline$>40$ & Obese class III (severely obese) \\
\hline
\end{tabular}

Table 1.1 BMI classifications, as given in (Wass and Owen, 2014).

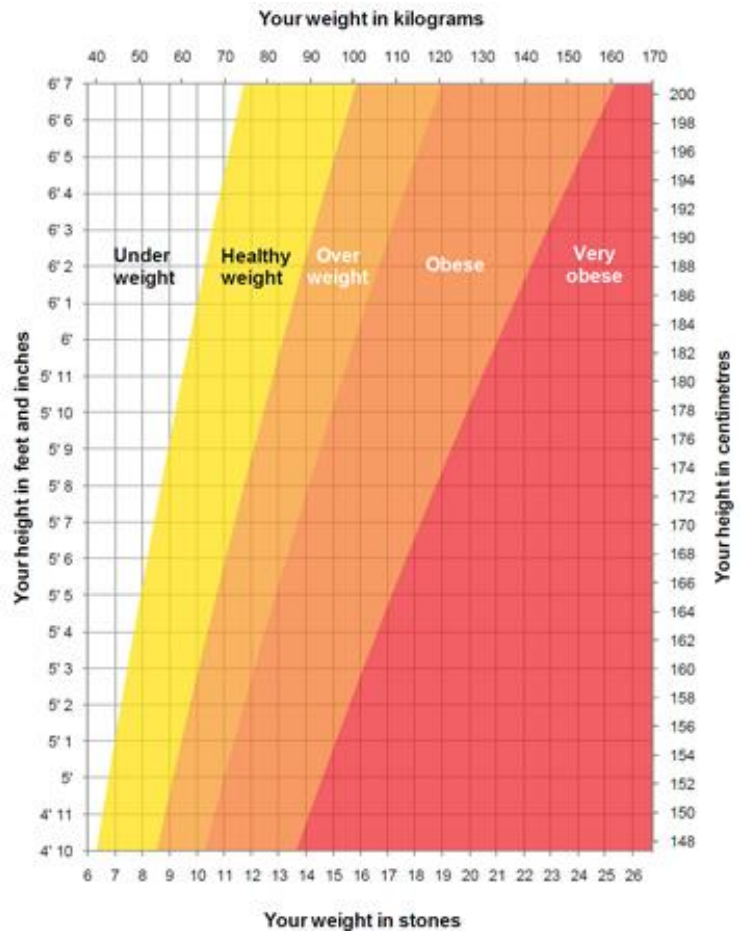

Figure 1.1 BMI Graph. http://www.nhs.uk/Livewell/healthy-living/Publishinglmages/weight-chart- 


\subsubsection{Environmental and genetic causes of obesity}

Obesity is a multifactorial disease that encompasses both environmental and genetic factors. Environmental factors are often highlighted within developed countries, where a higher caloric diet including refined sugars and a high fat content, alongside a sedentary lifestyle have become common place.

Obesity can be inherited with strong association of genetic factors, both monogenic and polygenic factors, for example, resulting in leptin deficiency or ghrelin abnormalities. Prader- Willi syndrome (PWS) is a key example of genetic obesity and results from a loss of paternal genes in the q11-13 region of chromosome 15. Patients with PWS experience excessive appetite (hyperphagia) which is linked to hyperghrelinaemia (DelParigi et al., 2002).

\subsubsection{Comorbidities linked to obesity}

The hypercaloric state associated with obese individuals has a detrimental effect on the individual's health and predisposes them to many diseases, including an increase risk in CVD, hyperlipidemia and T2D (Scerif et al., 2011). These comorbidities present a great challenge to the National Health Service (NHS) and healthcare providers, and has resulted in the practice of various bariatric surgeries to allow a reduction in adipose mass. Abdominal obesity causes a chronic low grade inflammatory state, activating the immune system and playing a vital role in obesity-related insulin resistance and T2D (Cooke et al., 2016). In an obese state, the make-up of adipose tissue changes, resulting in the infiltration of macrophages 
and the enlargement of adipocytes. Macrophages are responsible for the release of free fatty acids (FFA) and the subsequent release of tumour necrosis factor $\alpha$ (TNFa) (Bastard et al., 2006). FFA activate nuclear factor kappa B (NFkB) by binding to toll like receptor-4 on macrophages. Macrophage-derived TNF $\alpha$ activates adipocytes, inducing the expression of various genes and the release of adipokines (i.e. interleukin-6). The overproduction of adipokines produced by the infiltration of macrophages in the obese state is involved in the pathophysiology of insulin resistance (Bastard et al., 2006).

\subsection{Oxidative Stress}

Oxidative stress is the result of free radical mediated damage that often has severe pathological consequences. Free radicals are atoms or molecules which have one or more free electrons within the outer shell of the atomic structure, causing it to become unstable and reactive. The most common form of free radical mediated damage results from reactive oxygen species (ROS) (Stephens et al., 2009). Oxidative stress is often underpinned by a disturbance in the balance of pro- and anti-oxidants, resulting in a shift towards a pro-oxidant state. For instance, ROS are produced naturally in the body, but when over production occurs, together with insufficient anti-oxidant defences, it can be associated with pathological disorders i.e. diabetes and cancer (Stephens et al., 2009).

The development of ROS can result from the electron transport chain 'leaking' electrons, that combine with oxygen causing the formation of superoxide 
molecules (Evans et al., 1997, Cooke et al., 2003). As well as electron leaking, ROS formation can result from ionisation, ultraviolet radiation and certain exogenous chemicals. Due to the unstable nature of ROS they can potentially target all molecules in the body, especially DNA, attacking the double bonds present between bases, resulting in a direct effect on replication and transcription (Cooke et al., 2003).

\subsubsection{Lipid peroxidation}

Lipid peroxidation is the degradation of lipids within an environment of high oxidative burden. It is the main molecular mechanism that is involved in oxidative damage of cell structure resulting in the destruction of membrane lipids. Oxidative damage results from a free radical chain reaction composed of three stages; initiation, propagation and termination (Ayala et al., 2014). Initiation involves the formation of lipid radicals via the hydrogen abstraction or addition of an oxygen radical. Polyunsaturated fatty acids undergo peroxidation forming a conjugated diene which binds an oxygen molecule to produce a peroxyl radical. Thus, allowing for the propagation of the free radical chain reaction; peroxyl radicals producing hydroperoxides, capable of hydrogen abstraction of another polyunsaturated fatty acid. The free radical chain reaction is terminated when two free radicals conjugate or in the presence of a chain breaking anti-oxidant (Repetto et al., 2012, Ayala et al., 2014). 
The anti-oxidant effect can either be enzymatic or non-enzymatic. Enzymatic routes are often underpinned by anti-oxidant systems like superoxide dismutase (SOD), catalase or glutathione peroxidase (Valko et al., 2007). Non-enzymatic antioxidants consist of vitamin C, vitamin E and glutathione (GSH) (Valko et al., 2007). All anti-oxidant systems demonstrate a potent role in either the prevention, repair and/or termination and neutralization of ROS through reduction (Valko et al., 2007). Furthermore, increased lipid peroxidation within inflammatory diseases is a result of the promotion of ROS production (Repetto et al., 2012).

\subsubsection{Inflammation and ROS production}

Inflammation is the body's immune reaction in response to a pathogen, allowing it to eradicate the imposing threat. The process of inflammation is regulated by a variety of factors and is characterised by the production of pro-inflammatory cytokines and inflammatory cell activation. The activation of inflammatory cells results in an increase in superoxide and other ROS production, via the pro-oxidant action of the phagocytic isoform of nicotinamide adenine dinucleotide phosphate (NADPH) oxidase (Valko et al., 2007). Increased level of ROS can result in the alteration of inflammatory cell signalling pathways; activating c-Jun $\mathrm{N}$-terminal kinase (JNK), mitogen-activated protein kinase (MAPK), extracellular signalregulated kinase (ERK) and NFKB signalling pathways. The production of hydrogen peroxide acts as a secondary messenger that activates NFKB via TNF $\alpha$ and interleukin 1 (IL-1) (Valko et al., 2007). The generation of ROS within the immune system acts as a defence mechanism, however, as previously mentioned, if the rate 
of ROS production overwhelms the anti-oxidant system then there will be an increase in oxidative stress and lipid peroxidation.

\subsubsection{Oxidative stress in T2D}

Oxidative stress is implicated in the pathophysiology of comorbidities linked to T2D, due to increases in ROS and decreases in free radical quenching enzymes (Tangvarasittichai, 2015, Martín-Gallán et al., 2003). Hyperglycaemia activates detrimental pathways including the hexosamine pathway and AGE pathway, both of which cause an increase in oxidative stress (Figure 1.2). As previously mentioned, pancreatic $\beta$-cell function plays a vital role in the development of T2D. Chronic hyperglycaemia is reported to be a causative factor in $\beta$-cell apoptosis via oxidative stress, with a resultant decrease in $\beta$-cell mass (Valko et al., 2007). Anti-oxidant treatment is beneficial in the treatment of diabetes, resulting in the suppression of $\beta$-cell apoptosis and theorised protection against the loss of insulin mRNA and insulin concentration (Valko et al., 2007). 


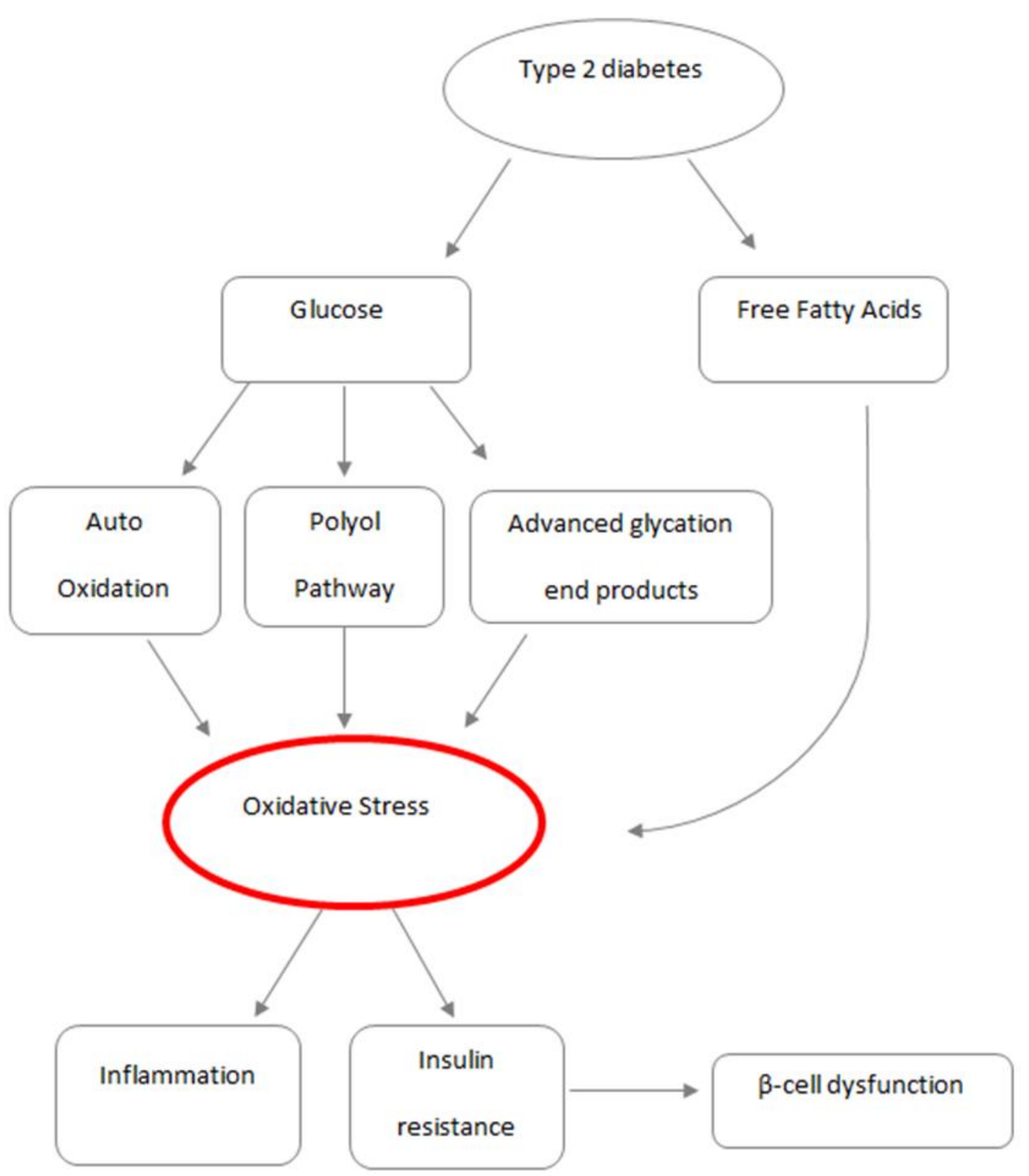

Figure 1.2. Overview of oxidative stress within Type 2 diabetes. Type 2 diabetes encompasses a raise in circulating blood glucose and free fatty acids. Both of which result in an increase of oxidative stress i.e. activation of auto oxidation, polyol and advanced glycation end product pathway in hyperglycaemia. An increase in oxidative stress can result in increased inflammation and insulin resistance triggering $\beta$-cell dysfunction. 


\subsection{Adipose tissue}

Adipose tissue is a highly complex and metabolically active organ. This endocrine organ has demonstrated a role in central nervous system (CNS) communication, fasting FFA source and regulation of nutrient homeostatic mechanisms (Trayhurn and Wood, 2004). Traditionally grouped into two simple classifications; white adipose tissue (WAT) and brown adipose tissue (BAT), a third classification has recently been discovered, 'beige' or 'brite' (Rosen and Spiegelman, 2014). All three classifications store energy in the form of lipid droplets, yet are distinct and contain differing gene expression patterns, allowing for specific differences in their functions and depots. WAT, beige adipose tissue and BAT all require peroxisome proliferator-activated receptor $\gamma$ (PPAR $\gamma$ ) for development and functionality (Rosen and Spiegelman, 2014, Harms and Seale, 2013). However, they differ in cell lineage (Figure 1.3) with embryonic stem cells that express key developmental and transcriptional factors Myf5 and Pax7 being the precursor for BAT and two distinct lineages Pax7 and Myf5 negative as the precursors for WAT and beige adipose tissue (Wu et al., 2013, Rosen and Spiegelman, 2014). 


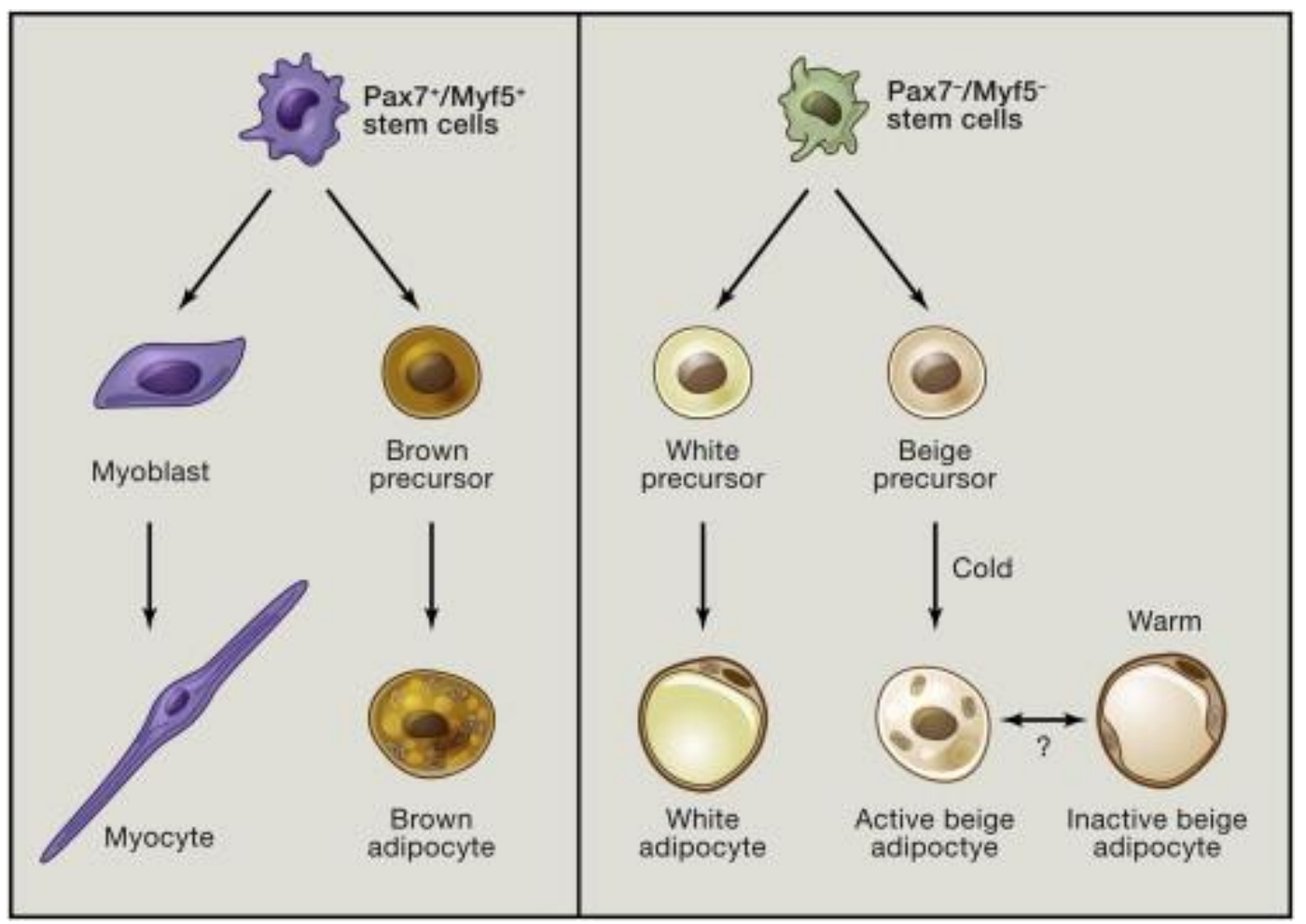

Figure 1.3. Different cell lineages that produce brown, beige and white adipocytes. Taken from Rosen and Spiegelman (2014)

\subsubsection{Brown adipose tissue}

BAT is a group of highly specialised cells that has vast vascularisation and cytochromes, with a main function of thermoregulation. BAT has the ability to transform stored energy into heat through the action of uncoupling protein 1 (UCP1). Located in the mitochondrial membrane, UCP1 catalyses a proton leak across the inner mitochondrial membrane, uncoupling fuel oxidation for ATP synthesis (Rosen and Spiegelman, 2014). BAT develops during gestation in humans, allowing for protection against cold present at birth and atmospheric life. Significant BAT depots present in gestation are depleted in adult humans, who instead are dependent upon shivering thermogenesis to maintain 
thermoregulation. Acute cold exposure results in sympathetic nervous system release of noradrenaline and the induction of BAT thermogenesis via the stimulation of intracellular lipolysis (Cypess et al., 2015).

As well as thermoregulation, chronic BAT stimulation has been highlighted to play a beneficial role in the regulation of metabolism (Harms and Seale, 2013). The activation of BAT is negatively associated with obesity and insulin resistance, hence a negative association with T2D (Orava et al., 2011). High levels of the glucose transporter 4 (GLUT4) are present within BAT, resulting in an increase in insulin sensitivity and glucose uptake (Cypess et al., 2015, Stanford et al., 2013, Orava et al., 2011)

\subsubsection{White adipose tissue}

WAT is an abundant reservoir of triglycerides which accounts for $85 \%$ of tissue mass. Spanning the human body, this heterogenous tissue is composed of mature adipocytes, fibroblasts, macrophages, endothelial cells and pre-adipocytes. The classical functions of WAT are lipid synthesis (lipogenesis), lipid storage and triglyceride breakdown. WAT also allows for the subsequent release of fatty acids under certain metabolic stressors i.e. exercise or caloric restriction.

WAT is linked to primary roles within the endocrine system, namely the secretion of adipokines. Adipokines are a group of diverse protein signals and factors that allow WAT to communicate throughout the body, having an effect on endocrine, paracrine and autocrine systems (Figure 1.4). This ability for adipokines 
to act either locally or distally allows for WAT to play an intrinsic role in the regulation of whole body energy homeostasis. However, not all regions of WAT produce the same amounts of adipokines resulting in various functions for anatomical fat depots.

\section{Retinol binding protein 4}

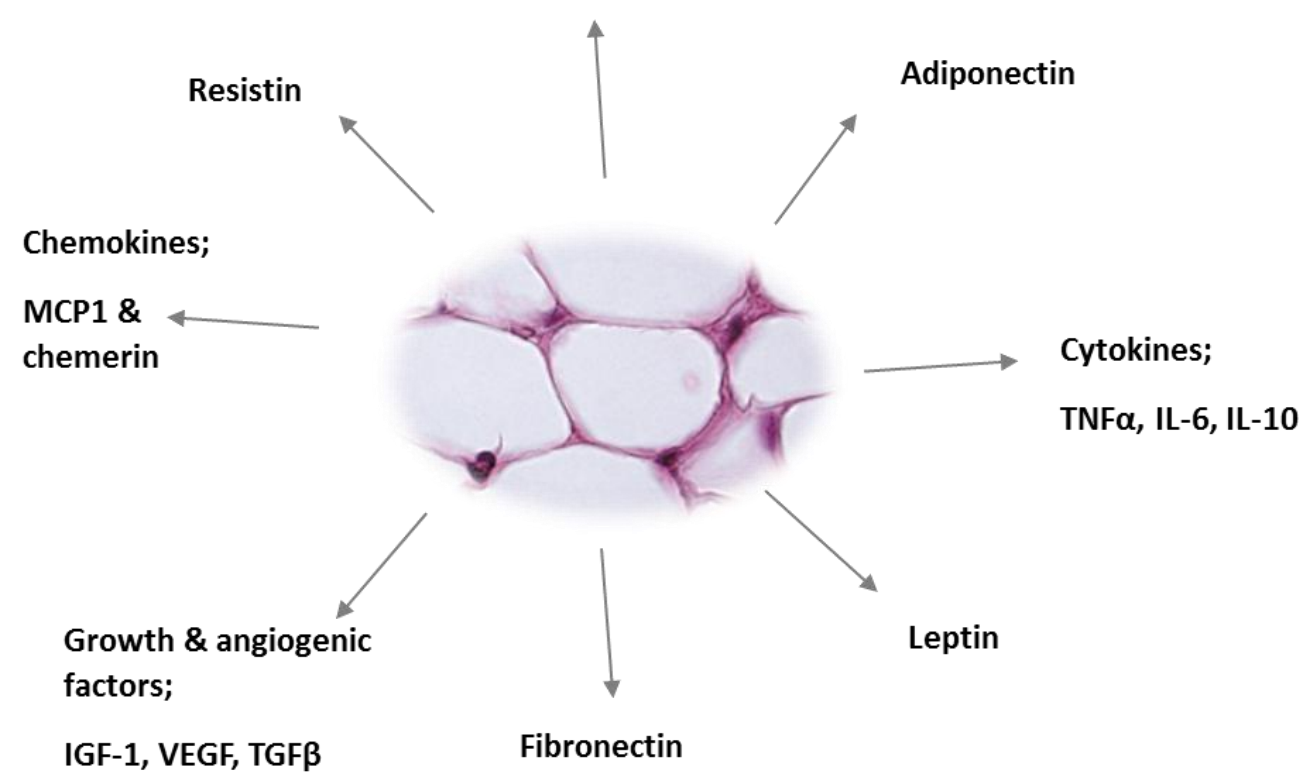

Figure 1.4. An example of adipokines secreted by white adipose tissue. Monocyte chemotactic protein (MCP), tumour necrosis factor (TNF), interleukin (IL), insulin-like growth factor (IGF), vascular endothelial cell growth factor (VEGF) and transforming growth factor (TGF).

\subsubsection{Beige adipose tissue}

Originally, WAT and BAT were thought to be located in anatomically separate regions, however over 30 years ago UCP1 positive cells were located in WAT depots (Cousin et al., 1992, Young et al., 1984). Beige adipose tissue originates from a 
different source to BAT and is the product of WAT differentiation, which can occur due to cold exposure or in the presence of key peptides e.g. neuropeptide Y. Often termed 'inducible BAT', beige adipose tissue and BAT share functionality and cellular morphology, however they exhibit differing gene expression. Basal UCP1 levels in beige adipose tissue is low and comparable to WAT, however cyclic adenosine monophosphate (CAMP) stimulation in vivo results in a rise of UCP1 expression equivalent to that of interscapular BAT (Wu et al., 2013). This indicates beige adipose tissue is unique in functionality, allowing energy to be stored as lipid droplets, as well as responding to cAMP, resulting in heat production and energy dissipation (Rosen and Spiegelman, 2014). Beige adipose tissue is inversely associated with age and obesity, and is increased after weight loss from bariatric surgery (Wu et al., 2013).

\subsubsection{Adipose depots}

Commonly, the location of WAT spans the inguinal, retroperitoneal and gonadal regions of the body whilst BAT is located in the interscapular, perirenal, axillary and paravertebral regions. Interscapular BAT has been identified in human adults using [18F]-2-fluoro-D-2-deoxy-D-glucose (FDG) and positron emission tomography (PET); further analysis of a biopsy section confirmed the tissue to have BAT morphology and characteristics, including UCP1 positivity (Rosen and Spiegelman, 2014, Cypess et al., 2009, van Marken Lichtenbelt et al., 2009, Virtanen et al., 2009). 
A large accumulation of WAT has been observed in two depots; subcutaneous located under the skin and visceral, largely in the omentum. The storage of adipose tissue in these depots plays a vital role in metabolic disease, an increase of visceral fat in obesity is associated with related diseases including T2D, however subcutaneous fat does not share this association.

\subsubsection{Adipogenesis}

Pre-adipocytes have the ability to proliferate and undergo differentiation into mature adipocytes, a process intensely regulated by specific transcription factors and cell cycle proteins. During adipogenesis, pre-adipocytes undergo a morphological change, resulting in a change from a fibroblastic to a spherical shape. As a result of the morphological changes, cellular make up is also altered, including quantitative changes in extracellular matrix and cytoskeletal components (MorenoNavarrete and Fernández-Real, 2012). Due to a key part of adipose tissues ability to have homeostatic system control, adipose differentiation and understanding of the transcriptional basis of adipogenesis has gained great importance.

PPARY and sterol-regulatory element binding protein-1 (SREBP-1) are key transcriptional factor in adipognesis. PPARY is a subfamily of nuclear hormone receptors and exists in two protein isoforms; $\operatorname{PPAR} \gamma_{1}$ and $\operatorname{PPAR} \gamma_{2}$, which are produced via alternative promoter usage and alternative splicing at the $5^{\prime}$ end of the gene (Wu et al., 1995). Present in high levels in pre-adipocytes, PPAR $y$ has a role as the master regulator of adipogenesis, inducing fat cell differentiation. PPARY 
adipogenic effect is reliant upon its transcriptional activity, where it is shown to increase transcription via the DNA recognition site DR-1 (Spiegelman and Flier, 1996).

SREBP-1 is encoded by the SREBF1 gene which is transcribed into two splice variants, SREBP-1a and SREBP-1c (Knebel et al., 2012). The sterol response binding protein exhibits two main functions in adipocytes characteristics. Firstly, it is shown to control de novo lipogenesis via the induction of key genes of fatty acid metabolism; whilst on the other hand, under conditions favouring adipogenesis SREBP-1 has shown to promote and increase the overall percentage of preadipocytes that undergo differentiation (Kim and Spiegelman, 1996, Knebel et al., 2012).

\subsubsection{Lipogenesis}

De novo lipogenesis (DNL) is the enzymatic process by which lipid synthesis occurs from acetyl CoA, predominantly within the liver but also within adipose tissue (Knebel et al., 2012, Kersten, 2001). The rate at which DNL occurs is diet dependent, with an increase in dietary carbohydrates resulting in increased lipogenesis and a subsequent rise in plasma triglycerides. Alternatively, fasting can cause a decrease in DNL within WAT; which when combined with an increase in lipolysis results in a loss of triglycerides. The radicalised triglycerides from WAT are transported to the liver where triglyceride synthesis is increased to overcome the influx, resulting in hepatosteatosis (fatty liver) (Kersten, 2001). 
DNL is a highly regulated process, induced from a variety of mechanisms including the increase in lipogenic enzymes and several transcription factors (Knebel et al., 2012). As previously mentioned, SREBP-1c is a key inducer of DNL due to its regulatory role in the expression of genes that encode vital lipogenic enzymes. Two central enzymes for DNL are acetyl CoA carboxylase and fatty acid synthase, and are responsible for the generation of fatty acids from the products of glycolysis (Figure 1.5). Insulin stimulates SREBP-1c levels in the liver, therefore in a high insulin environment i.e. obesity and T2D, there will be an increase in DNL that may result in dyslipidaemia. Liver $X$ receptor (LXR) response elements within the SREBP-1c promoter are identified as mediators of insulin induced SREBP-1C transcription (Howell et al., 2009). As well as DNL, SREBP-1c and LXR have demonstrated a role within other lipid-mediated pathways i.e. cholesterol metabolism (Wagner et al., 2003, Vaughan and Oram, 2005). 


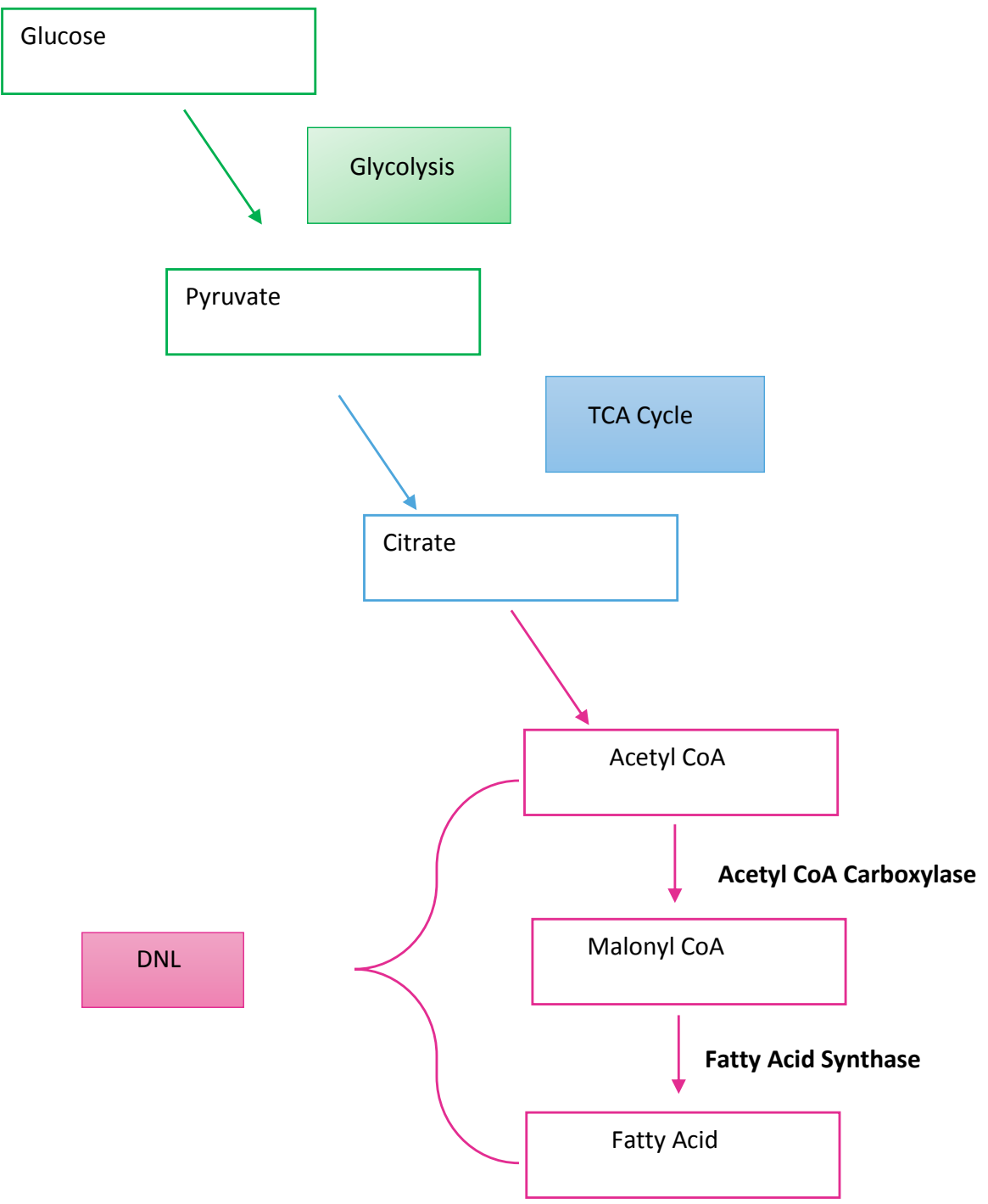

Figure 1.5. Pathway of fatty acid production. Glycolysis (GREEN), TCA cycle (BLUE) and de no lipogenesis (PINK) with key lipogenic enzymes in bold.

\subsubsection{Reverse cholesterol transport}

The reverse cholesterol transport pathway (Figure 1.6), the removal of excess cholesterol from peripheral tissues back to the liver for excretion and catabolism, is critical in the defence against atherosclerosis (Kennedy et al., 2005, Fielding and 
Fielding, 2001, Vaughan and Oram, 2005). Cholesterol efflux is dependent upon ATP binding cassette (ABC)A1 and ABCG1 (Kennedy et al., 2005, Ma et al., 2014). ABCA1 and ABCG1 are members of a superfamily of transporters that function to mediate the transfer of cellular cholesterol to the cell surface for removal by high density lipoproteins (HDL) (Kennedy et al., 2005, Vaughan and Oram, 2005). PPARy induces the expression of $A B C A 1 / G 1$ via nuclear cholesterol sensors, LXR $\alpha$ and LXR $\beta$ (Vaughan and Oram, 2005). LXR acts as a transcription regulator for the genes associated with cholesterol efflux, which is activated when total cellular cholesterol levels are high (Wagner et al., 2003). However, when total cellular cholesterol levels are low, SREBP induces cholesterol biosynthesis (Wagner et al., 2003).

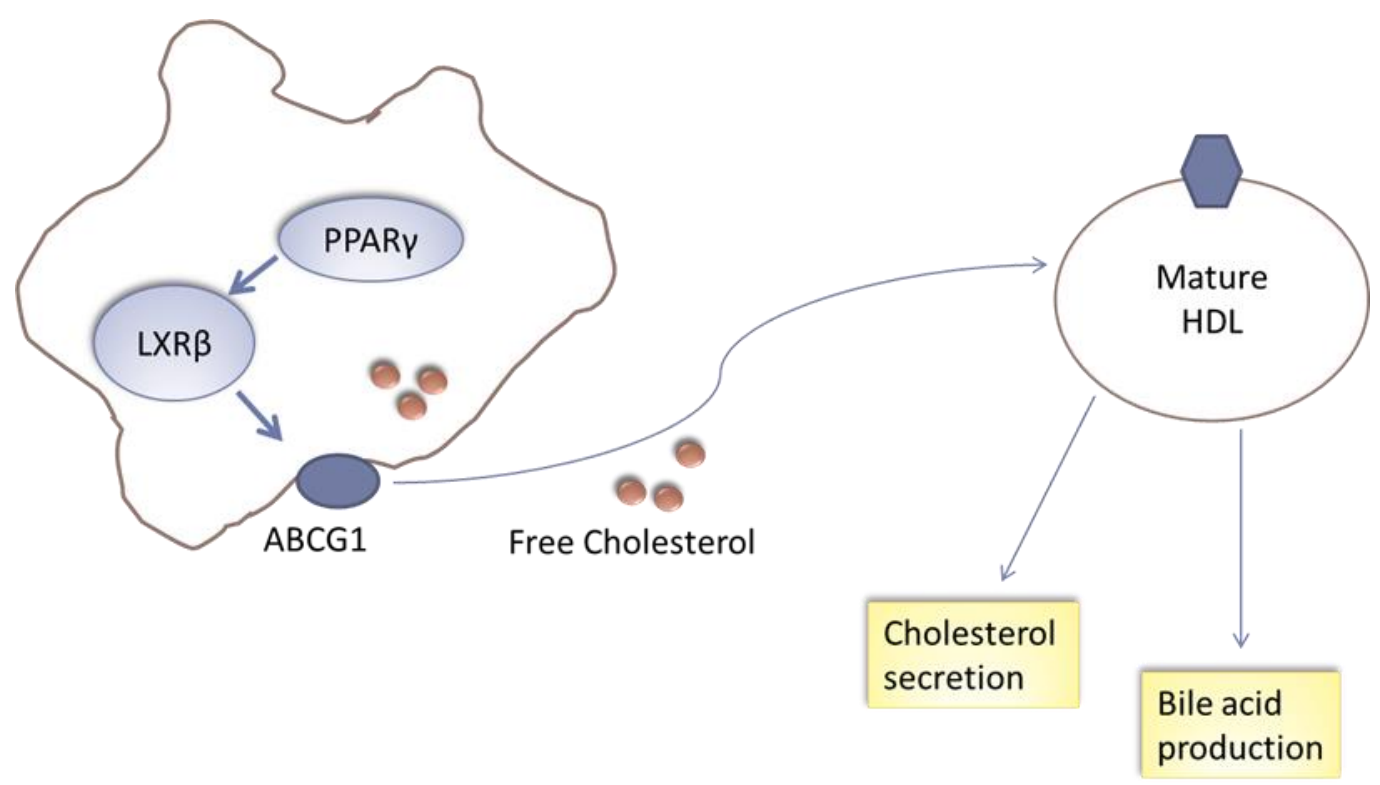

Figure 1.6. Reverse cholesterol transport within humans. Diagram illustrates key steps within the reverse cholesterol transport system in human macrophages. PPAR-LXR-ABC pathway exports intracellular cholesterol to the cellular membrane, mature HDL then transport free cholesterol to the liver where is undergoes cholesterol secretion or bile acid production. 


\subsection{Ghrelin}

\subsubsection{Discovery and structure}

In 1997, Kojima attempted to identify the unknown endogenous ligand responsible for growth hormone secretion via the stimulation of the growth hormone secreting receptor (GHS-R). However, initial studies proved unsuccessful despite the development of several hundred assays (Kojima et al., 1999). It was not until 1998, when Kojima and Hiroshi combined their findings with the recently described orphan G protein-coupled receptor GPR38 that progress was made (Kojima and Kangawa, 2008, Kojima, 2008). Through the use of a stable cell line, which expressed GHS-R, Kojima and co-workers were able to establish a calcium increasing activity (Kojima et al., 1999). The monitored activity was focused to a stomach extract, showing a ligand with a molecular weight ranging between 3-4 kDa (Kojima and Kangawa, 2008, Kojima, 2008). Final characterization of a 3 kDa endogenous ligand was deemed to be ghrelin (Peino et al., 2000).

Ghrelin was finally isolated from the gut of humans and rats via cloning (Shiiya et al., 2002, Peino et al., 2000). The research produced a novel 28 residue peptide hormone that was an endogenous ligand of GHS-R (Kojima et al., 1999). An anomaly was identified in the peptide structure and explained by the presence of a hydroxyl group in the serine 3 molecule undergoing acetylation (Tokunaga et al., 2005). Kojima originally proposed this hypothesis of hydroxylation, through the use of high performance liquid chromatography (HPLC) experimental data. He discovered that natural ghrelin was eluted 10 minutes after the synthetic desacylghrelin, concluding the modification occurred via a hydrophobic moiety. Electron 
ionization mass spectroscopy (EI-MS) analysis concluded the moiety mass was $\mathrm{C}_{7} \mathrm{H}_{15} \mathrm{CO}$ (Kojima et al., 1999) (Figure 1.7).

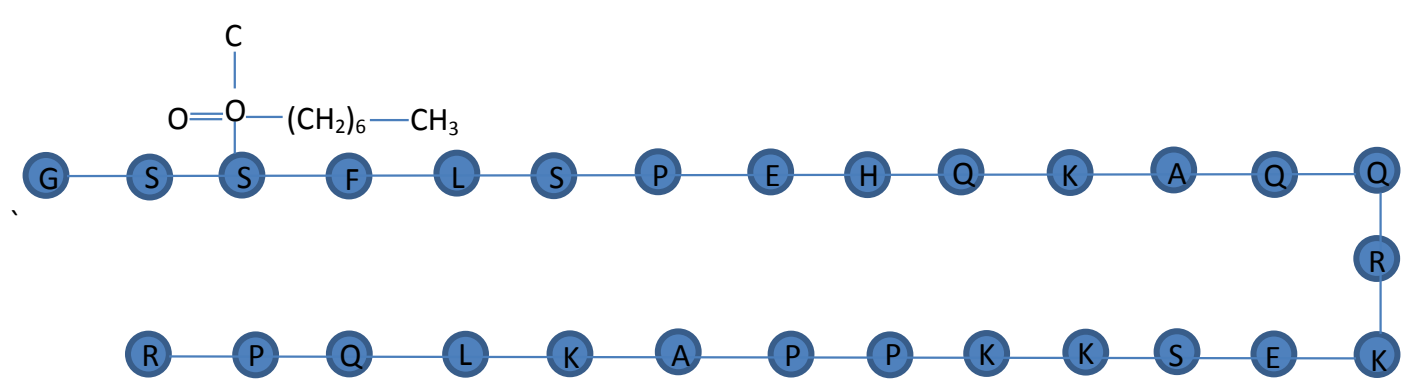

Figure 1.7 Amino acid sequence of ghrelin and the acetylation of the serine 3 residue.

The unique characteristics of ghrelin occur from the post translational state, where the peptide is originally synthesised as a pre-pro-hormone and proteolytically processed into the 28 amino acid sequence (Meier and Gressner, 2004). Pre-pro-ghrelin contains 117 amino acids. In addition to ghrelin, this gene encodes for a small signal peptide and obestatin. The cleavage of the pre-pro ghrelin gene produces a 23 amino acid signal and pro-ghrelin. Further proteolytic processing results in the cleaving of the 28 amino acid peptide by prohormone convertase $1 / 3$ (PC1/3) (Yang et al., 2008). As well as ghrelin, the presence of a second endogenous ligand has been reported in rat stomachs. This ligand is termed des-GLN 14-ghrelin and is a 27 residue peptide that is not processed from the ghrelin gene but a product of alternative splicing of the ghrelin gene mRNA, resulting in the loss of the 14th glutamine (GLN) residue (Figure 1.8) (Wang et al., 2002). The second endogenous ligand for GHS-R exists in the stomachs of all rats observed to date, suggesting that the endogenous ligands for GHS-R are two homologous peptides, des-GLN 14-ghrelin and ghrelin (Hosoda et al., 2000). 


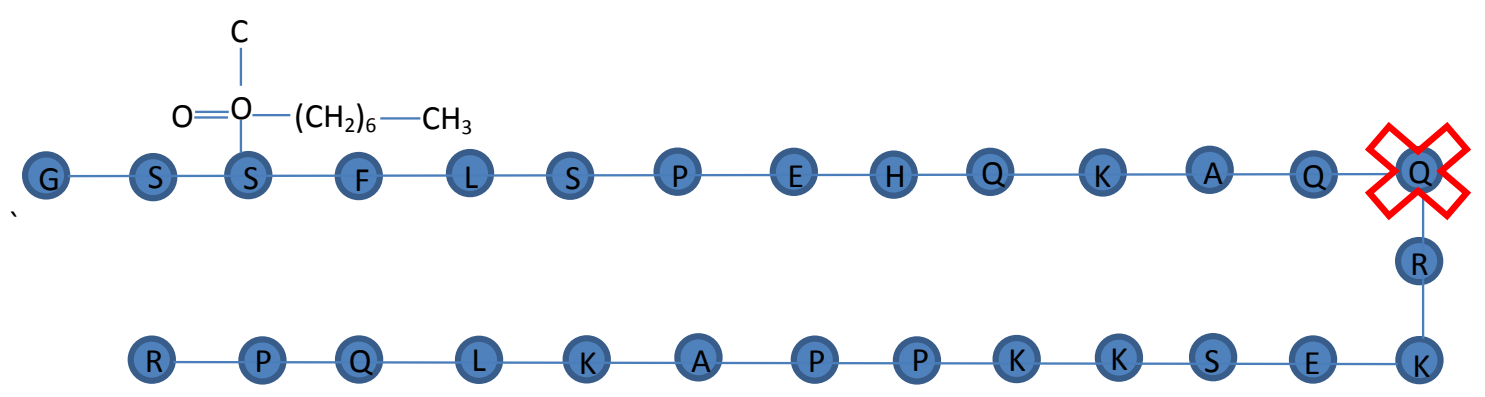

Figure 1.8. Ghrelin structure with a cross indicating the loss of an amino acid residue to produce desGLN 14-ghrelin via mRNA splicing.

Ghrelin is the first natural peptide to be reported containing the elusive post-translational modification of N-octanoylation (Kojima et al., 1999, Wang et al., 2002). In 2008, it was shown that a membrane bound O-acyltransferase 4 (mBOAT4) was vital in the activation of ghrelin (Kirchner et al., 2012). The human gene $m B O A T 4$ is located on chromosome 8 (8p12), containing 6 exons. It was later termed ghrelin O-acyltransferase (GOAT) due to it being the only enzyme that acylates ghrelin (Figure 1.9) (Kirchner et al., 2012). This acyl modification occurs at the pro-ghrelin maturation stage of ghrelin. 


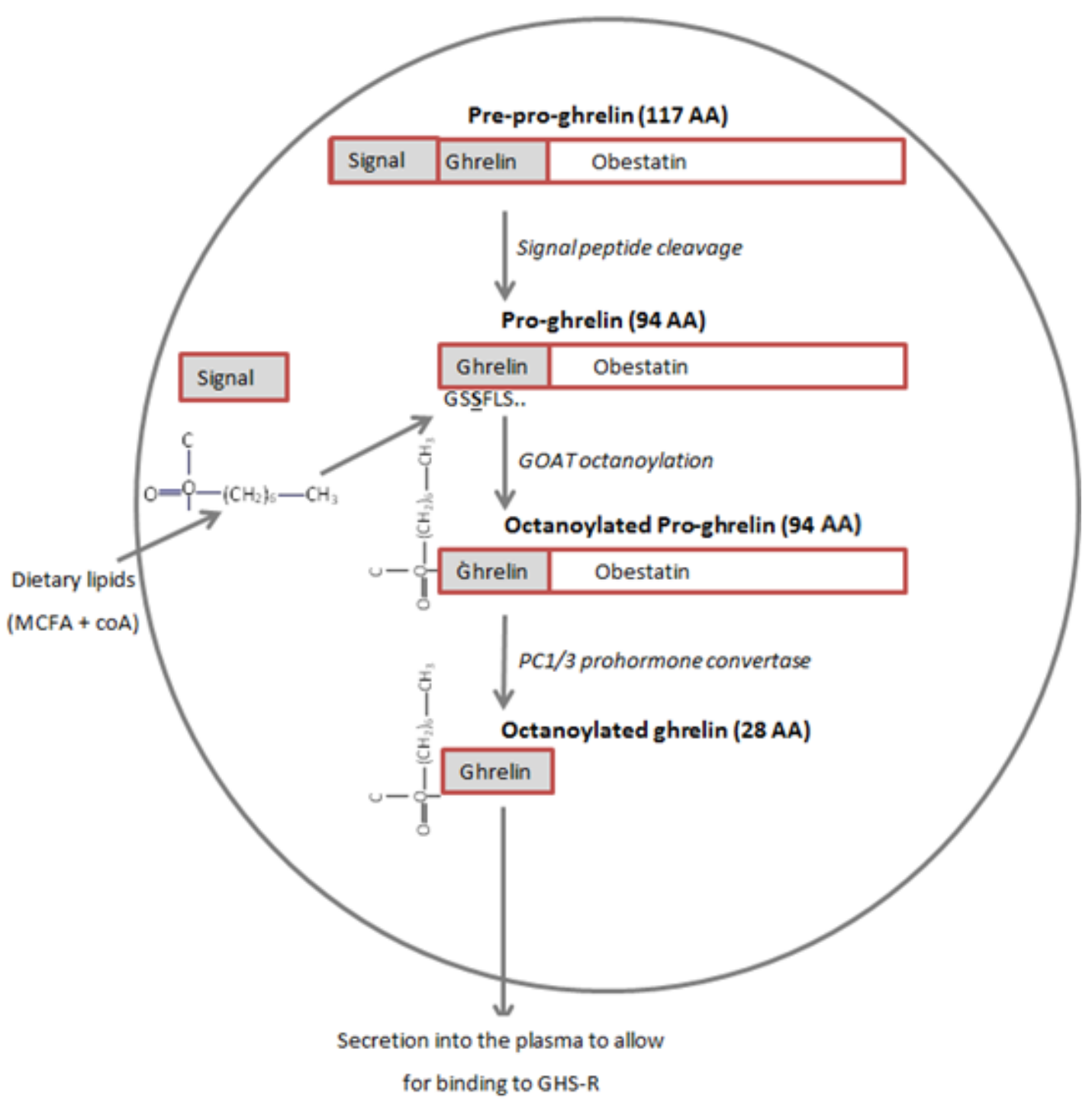

Figure 1.9. Ghrelin synthesis and maturation within a stomach cell. Amino acid (AA) length and name for each peptide given in bold and process undergoing or enzyme in italics. Medium chain fatty acids and acetly coA (MCFA + CoA) are the substrates used in the octanoylation of ghrelin by GOAT. Ghrelin also circulates in des acyl forms but only the acyl form is secreted to bind to GHS-R. 


\subsubsection{Function}

The peptide was named ghrelin, from the Latin word 'Ghre' which means 'grow' due to its role as a growth hormone releasing peptide (Meier and Gressner, 2004, Kojima and Kangawa, 2005). The novel peptide was isolated from the gut of both human and rat as the endogenous ligand of GHS-R (Kojima et al., 1999). GHS-R is transcribed in humans from the growth hormone receptor 1 (GHR1) gene, which encodes the full length functional receptor (GHS-R1 $\alpha$ ) and a splice variant truncated non-functional isoform (GHS-R1ß) (Gnanapavan et al., 2002, Müller et al., 2015). GHS-R1 $\alpha$ mRNA is expressed at low levels over a wide tissue distribution but is predominantly expressed in the anterior pituitary gland (Gnanapavan et al., 2002). The highest levels of ghrelin are secreted from the X/A-like cells of the oxyntic glands located in the gastric fundus, with lower levels widely distributed throughout the body (Garin et al., 2013, Dixit et al., 2004). Ghrelin is secreted directly into the local gastric circulation and transported to the brain directly, requiring it to either cross the blood-brain barrier via a saturated transport system or via the blood stream to enter areas of the brain that are not protected by the blood brain barrier (Angelidis et al., 2010).

Ghrelin also reaches its main active site, the hypothalamic arcuate nucleus (ARC), in an indirect manner, activating the vagus nerve and brain stem nuclei (Angelidis et al., 2010, Date et al., 2002). Ghrelin circulates in two major forms; acylated (approximately 5\% of total ghrelin) and desacyl (95\% of total ghrelin) (Rodriguez et al., 2009). GOAT is vital in the activation of ghrelin (Yang et al., 2008). Both forms of ghrelin are observed to cross the barrier in a blood to brain direction, 
however desacyl-ghrelin lacks the ability to cross the barrier in a brain to blood direction (Banks et al., 2002). Ghrelin's ability to cross the blood brain barrier is the result of non-saturable, saturable blood-brain transport and saturable brain-blood transport that is highly dependent on the unique post-translational acylation and primary structure (Serby et al., 2006, Banks et al., 2002, Banks et al., 2008). Ghrelin has a homeostatic role that encompasses multiple areas of the body, with actions that include; down regulation of BAT thermogenesis (Tsubone et al., 2005, Yasuda et al., 2003), modulation of non-hypothalamic brain regions producing an increased taste sensation (Cai et al., 2013) and stimulation of gastric emptying and motility (Masuda et al., 2000, Asakawa et al., 2003). In the last 16 years the peptide has generated considerable attention due to its role within these various peripheral effects (Müller et al., 2015)(Figure 1.10). 


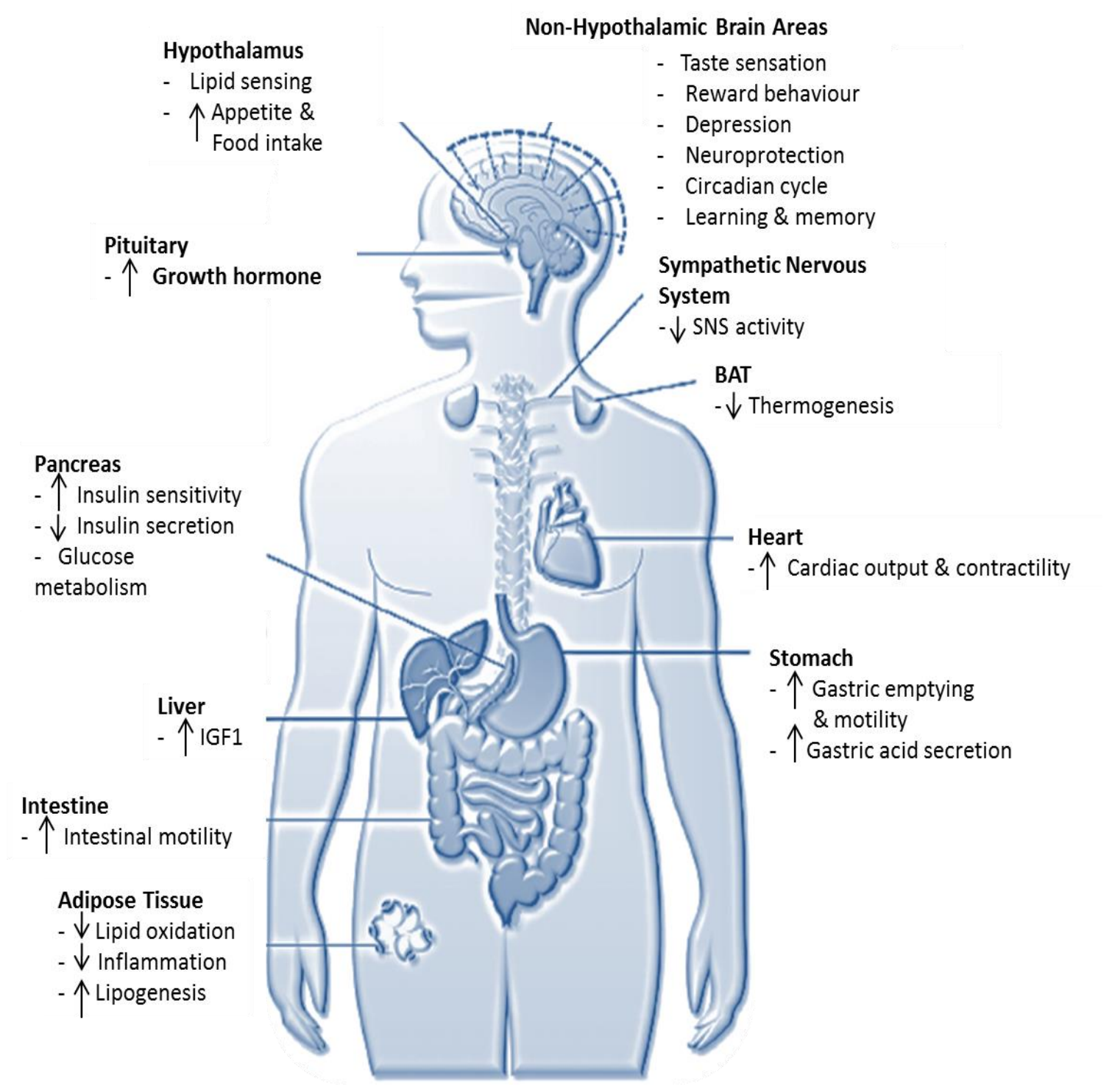

Figure 1.10 Cognitive and peripheral effect of ghrelin- taken from (Müller et al., 2015). Schematic indicates the effact of ghrelin administration on key areas of the body, importantly the effect on key tissues involveld in glucose homeostasis. Ghrelin is shown to alter glucose metabolism, increase insulin sensitivity and decrease insulin secretion in the pancreas, increase insulin-like growth factor 1 (IGF1) levels in the liver, decrease inflammation and increase lipogenesis in adipose tissue. 


\subsubsection{Acylation of ghrelin}

\subsubsection{GOAT acylation}

GOAT is responsible for the post-translational modification of ghrelin; the fatty acid modification of the amino acid structure in the peptide chain which is vital to determining functionality. As previously mentioned, ghrelin undergoes the attachment of an acyl moiety, octanoic acid, within the endoplasmic recticulum at the third serine residue following pre-pro-ghrelin cleavage into pro-ghrelin. GOAT is part of a 16 member family of membrane bound acyltransferases. This family, mBOATs, results in the transfer of long fatty acids to membrane-associated hydroxyl acceptors, however only one is responsible for ghrelin octanoylation, GOAT. GOAT expression is found widespread in human tissues, corresponding with that of ghrelin secreting tissues, thus GOAT expression levels are high in the stomach, gut and pituitary (Lim et al., 2011). The origin of the octanoyl (eight carbon) fatty acid, alternatively named medium chain fatty acid (MCFA), used in ghrelin activation is associated to orally ingested MCFAs. The location of the vast majority of ghrelin secreting cells are in the stomach lumen, allowing direct access to dietary MCFAs or medium chain triglycerols (Sato et al., 2012). Evidence shows the utilization of dietary MCFAs as a substrate, establishing GOAT as a dietary lipid sensor, however little is known about the comparison of de novo synthesised fatty acids used (Müller et al., 2015). 


\subsubsection{APT1 des-acylation}

In 2010, it was reported that acyl-ghrelin levels can be affected by acyl-protein thioesterase 1 (APT1). APT1 is encoded by the LYPLA1 gene, comprising of a 230 amino acid sequence homodimer. Originally APT1 was purified as a cytosolic lysophospholipid hydrolysing enzyme, the recombinant APT1 exhibits both deacylation and lysophospholipase activity (Satou et al., 2010). In contrast to GOAT, APT1 has been demonstrated to act as a ghrelin des-acylation enzyme (Shanado et al., 2004), thus suggesting that the levels of acyl-ghrelin present are dependent on the bioavailability of APT1 and GOAT (Satou et al., 2010).

\subsubsection{Ghrelin and adipogenesis}

Ghrelin is involved in the regulation of metabolic hormones with GHS-Rs present within adipose tissue (Korbonits et al., 2004, Kojima et al., 1999). In addition to stimulating GH secretion, ghrelin has been shown to play a role in adiposity. Upon the addition of acyl-ghrelin and desacyl-ghrelin, PPARY and SREBF1 mRNA expression levels increase in human visceral adipocytes during differentiation (Rodriguez et al., 2009). Therefore, in the presence of ghrelin, in vitro and in vivo studies have demonstrated that the mRNA levels of PPARY expression are increased, resulting in the differentiation of pre-adipocytes (Rodriguez et al., 2009). PPARy activity can be influenced by key components that play a role in the mammalian insulin pathway, such as mammalian target of rapamycin complex 1 (mTORC1) and Akt/protein kinase B (Akt/PKB) (Chabot et al., 2014). In the presence 
of ghrelin, mTORC1 and Akt/PKB can enhance PPARy activation promoting adipogenesis; demonstrating that ghrelin exhibits inter-relationships between adipogenesis and the insulin pathway (Chabot et al., 2014). The elevation of SREBF1 gene expression was accompanied by a significant increase in lipid accumulation in visceral adipocytes (Rodriguez et al., 2009). This administration of ghrelin directly stimulated the intra-cytoplasmic lipid accumulation via the increased production of various fat storage promoting enzymes including carboxylase, acetyl CoA, fatty acid synthase and lipoprotein lipase (Rodriguez et al., 2009).

\subsubsection{Ghrelin and lipogenesis}

Centrally acting ghrelin following chronic intra-cerebrovascular infusion, has also been shown to increase mRNA expression of genes which promote the retention of cholesterol (Davies et al., 2009). So in the presence of ghrelin, SREBP1-c expression is increased, with $A B C G 1$ and $\angle X R B$ expression decreased (Davies et al., 2009). Even though these results indicate that ghrelin has a role in increasing lipid retention within adipocyte cells, the relationship between cholesterol efflux, biosynthesis genes and ghrelin is disputed among studies. Sirtuin 1 and SIRT1 and tumour suppressor gene p53 activates AMP-activated protein kinase (AMPK) via phosphorylation, however in the presence of ghrelin there is the deacyetylation of p53 via SIRT1 impinging on AMPK activation (Velásquez et al., 2011, Kola et al., 2005). Phosphorylated AMPK inactivates fatty acid biosynthesis and activates fatty acid oxidation (Winder and Hardie, 1999). In vivo, the administration of ghrelin within p53 null mice demonstrates a decrease in lipid metabolism modulating gene 
expression, indicating that $\mathrm{p} 53$ is essential for the action of ghrelin on adipose tissue (Porteiro et al., 2013). Ghrelin administration has also been reported to activate the PPARY-LXR-ABC pathway in a dose-dependent manner, resulting in an increase in $L X R$ and $A B C$ expression in human THP-1 macrophages (Demers et al., 2009). An increase within cellular fat mass could result in an increase in lipogenesis and substrate uptake, and a decrease in lipolysis and cellular export (Mishra et al., 2016). These processes could alter the intrinsic regulation of FFA and cholesterol biosynthesis pathways that could lead to hypertriglyceridemia and other complications (Schultz et al., 2000). Due to the diversity of published data and various doses of acyl-ghrelin administered, further research into ghrelin-mediated lipid retention especially within humans is needed.

\subsubsection{Ghrelin and Type 2 diabetes}

An important contributor to the pathophysiology of T2D is the failure of glucose uptake into peripheral tissues such as adipose, skeletal muscle and liver. Decreased total ghrelin levels in patients with $\mathrm{T} 2 \mathrm{D}$ are associated with an increase in abdominal adiposity and insulin resistance (Katsuki et al., 2004). As previously mentioned, ghrelin has a demonstrated role in fat metabolism and glucose homeostasis; and cross-talk between lipid and glucose metabolism may result in a physiological role of ghrelin in insulin resistance. Cellular lipid accumulation that is observed upon ghrelin administration can have a knock on effect on glucose homeostasis. There are two hypotheses relating to lipid-mediated insulin resistance. The first is that an excess of visceral adiposity triggers release of FFA into 
the circulation. An increase in hepatic FFA oxidation triggers insulin resistance and an increase in glucose output from the liver (Lam et al., 2003, Boden, 1999). Acylghrelin infusion in humans has been associated with a rise in circulating FFA levels (Huda et al., 2009), and a subsequent decrease in insulin sensitivity (Boden, 1999). These findings demonstrate possible causation of insulin resistance that can occur in healthy volunteers when given an acyl-ghrelin infusion (Vestergaard et al., 2010, Vestergaard et al., 2007). The second hypothesis is that enlarged lipid-containing adipocytes are associated with the release of FFA, physical stress and ROS production (Maumus et al., 2008, Gustafson, 2010). Prolonged elevation of ghrelin increases visceral adiposity in mice and attenuates the transcription of LXRB and $A B C G 1$ which increases adipocyte volume due to a reduction in lipid export (Davies et al., 2009). This could alter immune function, as a result of increased ROS production and the release of damaging inflammatory agents such as TNF $\alpha$. This indirect immuno-modulatory response may lead to insulin resistance and T2D due to TNF $\alpha$ 's ability to induce the inhibitory phosphorylation of insulin receptor substrate (IRS)-1, leading to systemic insulin resistance (Gustafson, 2010). The association between obesity and T2D is well documented, allowing ghrelin to play a potential pharmacological role in its prevention and/or treatment.

\subsection{Aims}

I hypothesise that acyl-ghrelin will be shown to mediate lipid retention within human adipocytes, thus causing an increase in inflammation and oxidative stress. Upon translation from a single cell type to a depot, I propose that acyl-ghrelin would still be deemed detrimental to lipid homeostasis and thus would highlight 
key associations of acyl-ghrelin and inflammatory markers within the circulation of those with and without T2D. This thesis proposes to explore this hypothesis via the following aims:

1. Investigate the effect of acyl-ghrelin exposure on the lipid retention pathway and consequent inflammatory response, in an established human adipocyte cell line

2. Translate cell specific findings into a depot specific effect via analysis of the lipid retention pathway in human visceral adipose tissue biopsies

3. Explore acyl-ghrelin within circulating plasma of individuals with and without $\mathrm{T} 2 \mathrm{D}$ to establish any associations with indicators of the metabolic state 
Chapter 2

General Methodology 
This chapter is split into three arms to allow for fluidity throughout the methodology, the sections are based on result chapter's sample source and are as follows; (i) in vitro samples- cell lines, (ii) ex vivo samples- human visceral adipose tissue and (iii) cross over protocols, including data analysis.

\section{$2.1 \quad$ In vitro samples}

\subsubsection{Human pre-adipocyte cell line}

Alternative methodologies including primary human adipocytes and the excising of adipocytes from adipose tissue biopsies were also considered for the in vitro aspect of this project. Upon investigation of these techniques, the use of an established cell line was considered to be of greatest benefit for the experimental design of this project, allowing for experimental repeats and minimal risk of addition cell type carry over. However, cell lines can undergo alterations during high passage culture, therefore to reduce the risk of phenotypical changes within cells undergoing experiment the sub-culturing protocol restricted cell passages to a maximum of 10 .

A human pre-adipocyte cell line was derived from a stromal vascular fraction from infants with Simpson Golabi Behmel syndrome (SGBS) (Fischer-Posovszky et al., 2008). SGBS (MIM312870) is a rare $X$-linked congenital overgrowth syndrome to which the cause is not fully elucidative (Wabitsch et al., 2001). It has a clear genetic link with the glypican 3 gene, a gene involved in organ growth control, where often in cases of SGBS there is either a point mutation or deletion within the Glypican 3 gene (DeBaun et al., 2001). 
The human pre-adipocyte cell line is well established as a unique tool for studying adipocyte biology, exhibiting a high capacity for adipose differentiation that results in mature adipocytes. In 2001, the SGBS cell line was induced achieving adipogenic differentiation at a rate of over $90 \%$ for up to 50 generations (Wabitsch et al., 2001). The cell line exhibits specific metabolic functions; insulin-stimulated glucose uptake, insulin-stimulated de novo lipogenesis, $\beta$-adrenergic-stimulated lipolysis and adipokine secretion (Wabitsch et al., 2001, Fischer-Posovszky et al., 2008).

\subsubsection{Cell culture}

\subsubsection{Revival}

Upon arrival, a cryovial containing 1 million cells per $\mathrm{mL}$ was placed in a water bath at $37^{\circ} \mathrm{C}$ for rapid defrosting and placed in a falcon tube containing $20 \mathrm{~mL}$ of media $\mathrm{A}$ (Table 2.1 ) and $10 \%$ foetal calf serum (FCS) (Invitrogen ${ }^{\mathrm{TM}}$ ) to dilute freezing medium. The falcon tube was then centrifuged for 4 minutes at 1500 rotations per minute $(\mathrm{rpm})(200 \times \mathrm{g})$, supernatant decanted off and cell pellet resuspended in media $A$ plus $10 \%$ FCS and seeded into a T75 flask with $20 \mathrm{~mL}$ media A plus $10 \%$ FCS. Cells were incubated at $37^{\circ} \mathrm{C}$ in $5 \% \mathrm{CO}_{2}$. After 24 hours, media was decanted and refreshed and cells were incubated at $37^{\circ} \mathrm{C}$ in $5 \% \mathrm{CO}_{2}$. 


\begin{tabular}{|c|c|c|}
\hline Reagent & Quantity & Supplier \\
\hline DMEM F12 & $500 \mathrm{~mL}$ & Invitrogen $^{\mathrm{TM}}$ \\
\hline Panthotenate & $1.7 \mathrm{mM}$ & Sigma-Aldrich $^{\mathrm{TM}}$ \\
\hline Biotin & $3.3 \mathrm{mM}$ & Sigma-Aldrich $^{\mathrm{TM}}$ \\
\hline $\begin{array}{c}\text { Penicillin (10,000 } \mathbf{~} / \mathrm{mL}) / \\
\text { Streptomycin (10,000 } \\
\text { solution } / \mathrm{mL})\end{array}$ & $5 \mathrm{~mL}$ & Invitrogen $^{\text {TM }}$ \\
\hline
\end{tabular}

Table 2.1. Media A components

\subsubsection{Subculturing}

Cells were grown in T75 flasks until $80 \%$ confluent, old media was removed and cells washed three times with $4 \mathrm{~mL}$ phosphate buffered saline (PBS) (Corning ${ }^{\mathrm{TM}}$ ). Adhered cells were trypsinised using $2 \mathrm{~mL}$ of $0.05 \%$ Trypsin-EDTA (Gibco ${ }^{\circledR}$, Life Technologies ${ }^{\mathrm{TM}}$ ) and incubated for 5 minutes at $37^{\circ} \mathrm{C}$. Detached cells were suspended in $5 \mathrm{~mL}$ media and centrifuged for 10 minutes at $1500 \mathrm{rpm}$ ( $200 \times \mathrm{g}$ ) and the supernatant discarded. Cells were resuspended in $1 \mathrm{~mL}$ of media $A$ and counted using a Scepter ${ }^{\mathrm{TM}}$ 2.0, an automated handheld cell counter (EMD Millipore) and split into four T75 flasks at approximately $2,000,000$ cells in $20 \mathrm{~mL}$ media A containing $10 \%$ FCS. The flasks were incubated at $37^{\circ} \mathrm{C}$ and $5 \% \mathrm{CO}_{2}$ with a cell doubling time of $38.4 \pm 1$ hour; cells were split every $4-5$ days at $80 \%$ confluency. 


\subsubsection{Cell seeding}

SGBS cells at passage 5 were seeded into 6 -well plates for differentiation and treatments. Confluent T75 flasks were washed and trypsinised as detailed in section 2.1.2, $10 \mathrm{~mL}$ of media A was added to the T75 flask, transferred to a $50 \mathrm{~mL}$ falcon tube and centrifuged for 10 minutes at $1500 \mathrm{rpm}$ ( $200 \times \mathrm{g}$ ). Cells were counted and placed into 6 well plates at a seeding density of $0.2 \times 10^{6}$ with $1.5 \mathrm{~mL}$ media A containing $10 \% \mathrm{FCS}$. Cells were placed in a $\mathrm{CO}_{2}$ incubator at $37^{\circ} \mathrm{C}$ and $5 \% \mathrm{CO}_{2}$ until $90 \%$ confluent.

\subsubsection{Cell differentiation}

Media A was decanted from the 6 well plates and adhered cells were washed three times with PBS as described in section 2.1.2. Differentiation is induced under serum-free culture condition that contain a specific adipogenic cocktail made up of various components to aid in the differentiation of preadipocytes. Frequently found within adipogenic cocktails are insulin, dexamethasone and 3-isobutyl-1methylxanthine (IBMX) (Scott et al., 2011). Insulin acts to induce proliferation and differentiation of preadipocytes, as in high concentrations it is known to mimic insulin-like growth factor-1, activating mitogen-activated protein kinase pathways and in turn activating PPARY and thus adipogenic gene expression (Ailhaud, 1982, Qiu et al., 2001, Kim et al., 2010). Dexamethasone is an anti-inflammatory steroid molecule and IBMX is a competitive non-selective phosphodiesterase inhibitor, both of which regulate PPARy-promoting adipogenesis and induce transcription 
factors for growth and differentiation (Salasznyk et al., 2005, Gurriarán-Rodríguez et al., 2011). In addition, the adipogenic cocktail also contained rosiglitazone, a PPAR $y$ agonist that aids in sensitizing adipocytes to insulin action, increasing the rate and degree of differentiation (Ninomiya et al., 2010, Scott et al., 2011); thyroid hormone (T3), which stimulates basal metabolic rate, transferrin to act as an iron source for adipogenesis and cortisol to promote adipogenesis (differentiation media B).

Differentiation media B (2 mL)(Table 2.2) was introduced to each well for four days of incubation. On day 4, differentiation media B was removed via decanting and $2 \mathrm{~mL}$ of differentiation media $\mathrm{C}$ added (Table 2.3) for a further 10 day incubation. Fresh media was supplied every 4 days as previously published (Wabitsch et al., 2001) (Figure 2.1).

\begin{tabular}{|c|c|c|c|}
\hline Reagents & Quantity & Concentration & Supplier \\
\hline Media A & $10 \mathrm{~mL}$ & & See Table 2.1. \\
\hline Transferrin & $100 \mu \mathrm{L}$ & $0.01 \mathrm{mg} / \mathrm{mL}$ & Sigma-Aldrich $^{\mathrm{TM}}$ \\
\hline $\begin{array}{c}\text { Insulin } \\
\text { Cortisol }\end{array}$ & $2 \mu \mathrm{L}$ & $20 \mathrm{nM}$ & Sigma-Aldrich $^{\mathrm{TM}}$ \\
\hline $\begin{array}{c}\text { Triiodothyronine } \\
\text { (T3) }\end{array}$ & $10 \mu \mathrm{L}$ & $100 \mathrm{nM}$ & Sigma-Aldrich $^{\mathrm{TM}}$ \\
\hline $\begin{array}{c}\text { Dexamethasone } \\
\text { IBMX }\end{array}$ & $10 \mu \mathrm{L}$ & $0.2 \mathrm{nM}$ & Sigma-Aldrich $^{\mathrm{TM}}$ \\
\hline Rosiglitazone & $2 \mu \mathrm{L}$ & $250 \mu \mathrm{nM}$ & Sigma-Aldrich $^{\mathrm{TM}}$ \\
\hline
\end{tabular}

Table 2.2. Differentiation media B components 


\begin{tabular}{|c|c|c|c|}
\hline Reagents & Quantity & Concentration & Supplier \\
\hline Media A & $10 \mathrm{~mL}$ & & See Table 2.2 \\
\hline Transferrin & $100 \mu \mathrm{L}$ & $0.01 \mathrm{mg} / \mathrm{mL}$ & Sigma-Aldrich $^{\mathrm{m}}$ \\
\hline Insulin & $2 \mu \mathrm{L}$ & $20 \mathrm{nM}$ & Sigma-Aldrich $^{\mathrm{TM}}$ \\
\hline $\begin{array}{c}\text { Cortisol } \\
\begin{array}{c}\text { Triiodothyronine } \\
\text { (T3) }\end{array}\end{array}$ & $10 \mu \mathrm{L}$ & $100 \mathrm{nM}$ & Sigma-Aldrich $^{\mathrm{TM}}$ \\
\hline
\end{tabular}

Table 2.3. Differentiation media C components

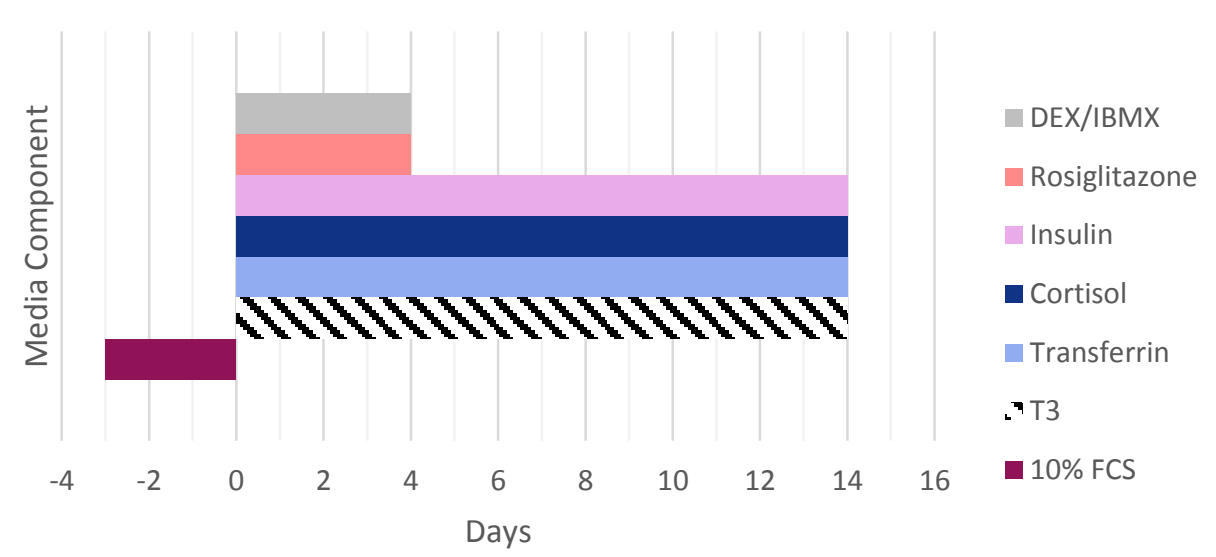

Figure 2.1. Media components for 14 day differentiation period

\subsubsection{Cell treatment}

Mature human adipocytes were exposed to various treatments as specified within each chapter. In Chapter 3, mature adipocytes were exposed to either an acute normo- or hyperglycaemic environment at day 12 of differentiation. The ranges of glucose added to the media were based on the physiological ranges present within 
individuals with Type 2 diabetes (T2D) and healthy individuals, respectively. To exhibit a normoglycaemic and hyperglycaemic environment, media A was spiked with D-glucose $\left(\right.$ Sigma $\left.^{\mathrm{TM}}\right)$ and set at glucose concentrations of $5.5 \mathrm{mM}$ and $25 \mathrm{mM}$, respectively. On day 14, mature adipocytes were exposed to either acyl-ghrelin (100 nM), lipopolysaccharide (LPS) $(1 \mu \mathrm{g} / \mathrm{mL})$ or LPS and acyl-ghrelin $(1 \mu \mathrm{g} / \mathrm{mL}, 100$ $\mathrm{nM}$ respectively), dependent on allocated well in plate layout (Figure 2.2). The collection of RNA and cell supernatant occurred at 17 and 34 hours post treatments.

Within Chapter 5, mature adipocytes were also exposed to a hyperglycaemic ( $25 \mathrm{mM}$ glucose) environment at day 12 . The cells were then dosed with metformin (SigmaAldrich $^{\mathrm{TM}}$ ) on day 14 . The cell dosage of metformin was determined by previously published literature (Section 5.3.1). Vehicle of PBS was used as a control within both Chapter 3 and Chapter 5 . Cell dosage concentrations were indicative of previous published work within similar cell types, as discussed in relevant chapter, to establish the effect of treatment and not representative of physiological level. 

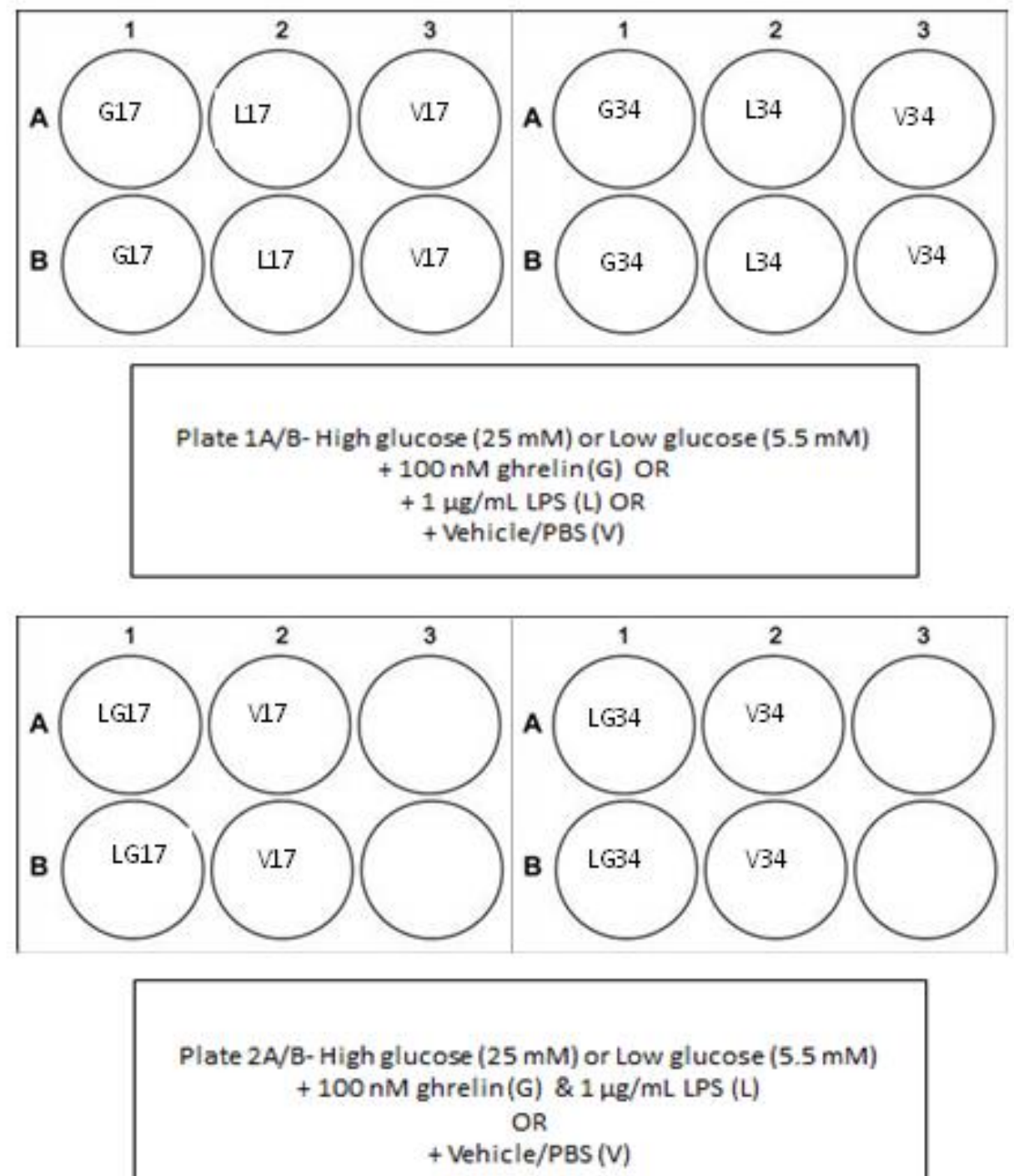

Figure 2.2 Plate layouts for cell treatments, replicated for high and low glucose concentrations. Plate one represents the wells treated with $100 \mathrm{nM}$ ghrelin (G) or $1 \mu \mathrm{g} / \mathrm{mL}$ LPS (L) or PBS (V). Plate 2 is combined treatment with ghrelin $(100 \mathrm{nM})$ and LPS $(1 \mu \mathrm{g} / \mathrm{mL})(\mathrm{GL})$ or PBS $(V)$. Wells will be treated for the time indicated in hours for each well; 17 or 34 hours.

\subsubsection{Oil red 0 staining}

At the required time points (17 and 34 hours) mature adipocytes underwent oil red O staining to allow for the quantification of accumulated lipid area (Figure 2.3). All 
reagents were purchased from Sigma Aldrich ${ }^{\mathrm{TM}}$ and staining undertaken as described by the manufacturer's protocol and published by Kishore and colleagues (Kishore et al., 2012).

\section{Fixing adipogenic cultures}

Treatment media was aspirated from the wells and adhered cells washed with PBS and fixed by the addition of $2 \mathrm{~mL} 10 \%$ formalin. After one hour of incubation at room temperature, the $10 \%$ formalin was removed, cells washed with deionised water and placed in $2 \mathrm{~mL} 60 \%$ isopropanol for 4 minutes then aspirated off.

\section{Preparation of Oil red O stain}

Stock solution was prepared by adding $300 \mathrm{mg}$ of Oil red O stain powder to $100 \mathrm{~mL}$ of $99 \%$ isopropanol, this stock solution was then diluted 3 parts solution in 2 parts deionised water and left to stand at room temperature for 10 minutes. The solution was then passed through a filter to remove any residual debris. Prepared oil red $\mathrm{O}$ solution is only stable for 2 hours so a fresh working concentration was required every use.

\section{$\underline{\text { Staining adipogenic cultures }}$}

Prepared Oil Red O solution $(500 \mu \mathrm{L})$ was added to each well whilst the plate was in oscillation, incubated at room temperature for 5 minutes and then washed with deionised water. Cells were then counterstained; $1 \mathrm{~mL}$ of Hematoxylin solution $\left(\right.$ Sigma $\left.^{\mathrm{TM}}\right)$ was added to the cells, left for 1 minute, then rinsed with warm deionised water. 


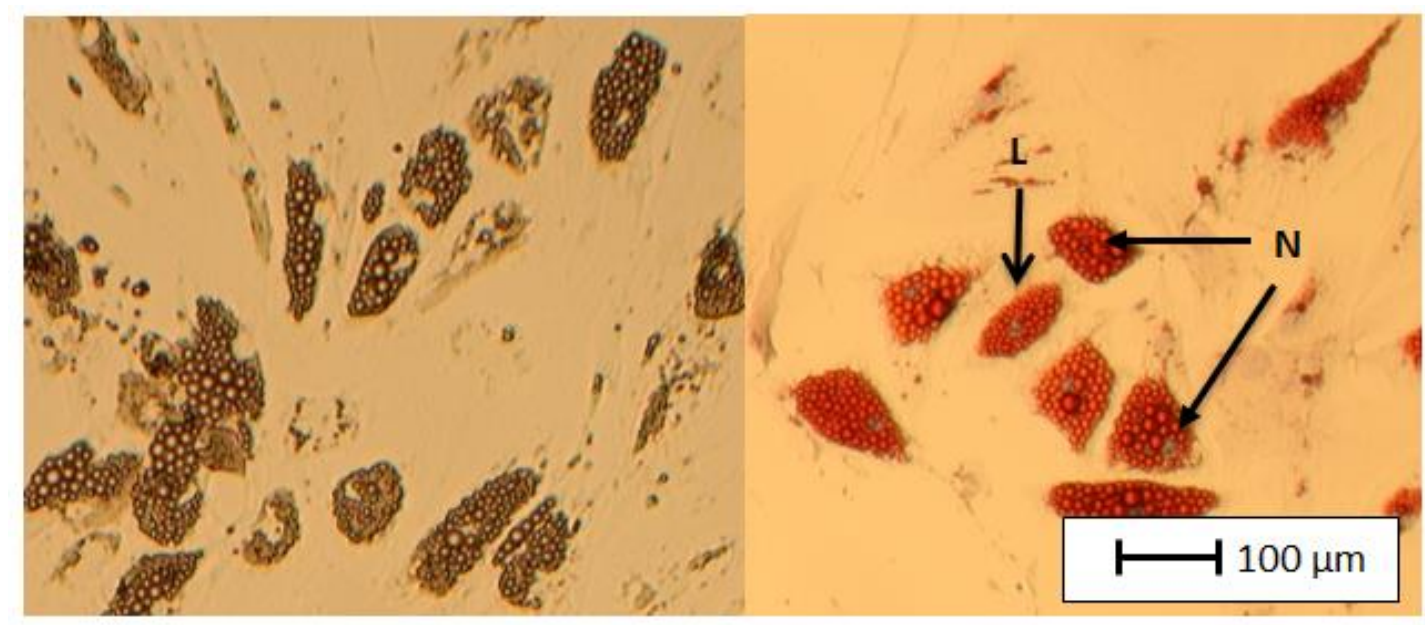

Figure 2.3. Mature SGBS cells imaged at magnification $x 50$ before and after Oil red $\mathrm{O}$ staining and haematoxylin counterstain. $\mathrm{N}=$ nucleus, $\mathrm{L}=$ lipid droplet.

\subsubsection{Cell supernatant collection}

Cell supernatant was aspirated off all treatment wells and collected in $2 \mathrm{~mL}$ centrifuge tubes, the supernatant then centrifuged at $4000 \mathrm{rpm}(500 \mathrm{xg})$ for $10 \mathrm{~min}$ at $4^{\circ} \mathrm{C}$. To ensure all cell debris was removed, the supernatant was placed in a fresh $2 \mathrm{~mL}$ centrifuge tube which was labelled with cell treatment, replicate identifier and time point for storage at $-20^{\circ} \mathrm{C}$ until needed.

\subsubsection{RNA extraction}

At collection time points, RNA was collected from the mature adipocytes using the RNAeasy mini kit (Qiagen ${ }^{\mathrm{TM}}$ ). To the allocated well, $350 \mu \mathrm{L}$ of buffer RLT was added and the cells dislodged using a cell scraper. Cell lysate was collected using a $1 \mathrm{~mL}$ pipette and placed into a $2 \mathrm{~mL}$ centrifuge tube. The sample was homogenised by 
passing through a $20 \mathrm{G}$ needle, 7 times. One volume of $70 \%$ ethanol was added to the homogenised sample and mixed well via pipetting. The next stage of the extraction used an RNAeasy mini kit (Qiagen ${ }^{\mathrm{TM}}$ ) which contains all components and buffers. The lysate was transferred to an RNAeasy mini column with a $2 \mathrm{~mL}$ collection tube and centrifuged at $13,000 \mathrm{rpm}(10,400 \times \mathrm{g})$ for 15 seconds and the flow through discarded. The collection tube was replaced with a clean $2 \mathrm{~mL}$ collection tube, $700 \mu \mathrm{L}$ of buffer RW1 added and centrifuged for 15 seconds at $13,000 \mathrm{rpm}(10,400 \mathrm{xg})$. The collection tube was discarded with flow through and replaced with a fresh $2 \mathrm{~mL}$ tube, $500 \mu \mathrm{L}$ of buffer RPE added and centrifuged for 15 seconds at $13,000 \mathrm{rpm}(10,400 \times \mathrm{g})$. This step was repeated a further time. RNA was eluted by placing the spin column into a clean $1.5 \mathrm{~mL}$ collection tube and $40 \mu \mathrm{L}$ of RNase-free water added and centrifuged for 1 minute at 13,000 rpm $(10,400 \times \mathrm{g})$. This step was repeated with a further $40 \mu \mathrm{L}$ of RNase-free water and incubated for 5 minutes to ensure all RNA was eluted. The column was centrifuged again for the final time for 1 minute at 13,000 rpm (10,400 x g).

\subsubsection{DNA free treatment}

In order to remove any genomic DNA contamination from the RNA, a DNA-Free Kit $\left(\right.$ Ambion $^{\mathrm{TM}}$ ) was used according to manufacturers' guidelines. DNase I buffer (10X) of 0.1 volume and $1 \mu \mathrm{L}$ DNase I was added to eluted RNA, vortexed and incubated at $37^{\circ} \mathrm{C}$ for 30 minutes. Then 0.1 volume of re-suspended DNase inactivation reagent was added to the mixture, vortexed and left to stand for 2 minutes' whilst mixing occasionally. Samples were centrifuged at 13,000 rpm for 1 minute and 30 seconds, when they were aliquotted into $20 \mu \mathrm{L}$ for storage at $-80^{\circ} \mathrm{C}$ until needed. 


\subsubsection{RNA quantification}

Extracted RNA was quantified using a NanoDrop spectrophotometer at $260 \mathrm{~nm}$ wavelength. The nanodrop allowed for the measurement of up to eight $1 \mu \mathrm{L}$ samples to be measured simultaneously. When the sample is placed on the pedestal, the arm is closed which forms a sample column and is held into place by surface tension during measurement. Before each measurement, the NanoDrop was blanked using DNA and RNA free water, $1 \mu \mathrm{L}$ sample was measured and RNA concentrations were recorded as $\mathrm{ng} / \mu \mathrm{L}$. As a measure of purity the RNA absorbance ratio at $260 / 280 \mathrm{~nm}$ was also recorded, a ratio of around 2.0 is generally accepted as 'pure' for RNA (Desjardins and Conklin, 2010).

\subsection{Ex vivo samples}

\subsubsection{Sample collection}

The East of Scotland Research Ethics Committee granted ethical approval for the project proposal (14/ES/1073)(Appendix 1). Sampling criteria required 10 participants as deduced from power calculation to achieve $80 \%$ power based on previous hVAT sample analysis with inflammatory marker IL-6 as a primary output, for each three groups:

I. Non-obese $\left(\mathrm{BMI}<30 \mathrm{Kg} / \mathrm{m}^{2}\right)$

II. Obese $\left(\mathrm{BMI}>30 \mathrm{Kg} / \mathrm{m}^{2}\right)$

III. Obese with Type 2 diabetes (BMI $>30 \mathrm{Kg} / \mathrm{m}^{2} \&$ T2D) 
The sample collections were carried out by myself at Morriston and Singleton Hospitals, Swansea. Eligibility inclusion criteria included between age 18-65 years, undergoing elective abdominal surgery and generally well at time of surgery, and exclusion criteria included unable to give informed consent and any participant undergoing surgery for an underlying malignancy. Informed consent was taken by myself after eligibility was checked by surgeon. Informed consent was given to collect human visceral adipose tissue (hVAT), two EDTA blood samples and additional clinical information. The participants' demographic measures (weight, height, birth date, blood pressure, medications and previous medical history) were documented from the medical records. Participants with systolic blood pressure $\geq 140 \mathrm{mmHg}$ and/or diastolic blood pressure $\geq 90 \mathrm{mmHg}$ or on antihypertensive medications were deemed hypertensive.

All participants information was anonymised with an unique study identification number. A GP letter was sent out and noted within the medical records that the participant had taken part within the 'Ghrelin' study. No further contact was required with the participant.

\subsubsection{Blood collection}

During the surgical procedure, two $4 \mathrm{~mL}$ Vacutainer ${ }^{\mathrm{TM}}$ EDTA-plasma tubes of whole blood were collected. $350 \mu \mathrm{L}$ of blood was transferred to cold sterile centrifuge tubes containing 4-(2-Aminoethyl)-benzenesulfonyl fluoreide (AEBSF) at a final concentration of $2 \mathrm{mg} / \mathrm{mL}$ and gently mixed to inhibit the activity of proteinases 
within 30 minutes of the blood being drawn. The blood containing AESBF was centrifuged at $6,000 \mathrm{rpm}(2,000 \times \mathrm{g})$ for 15 minutes at $4^{\circ} \mathrm{C}$ and the supernatant decanted into separate sterile tubes $\left(50 \mu \mathrm{L}\right.$ aliquots) and stored at $-80^{\circ} \mathrm{C}$ until needed. The remainder of the EDTA tube of whole blood was aliquoted into $1 \mathrm{~mL}$ centrifuge tubes and stored at $-20^{\circ} \mathrm{C}$. The second EDTA tube was centrifuged at $6,000 \mathrm{rpm}(2,000 \times \mathrm{g})$ for 5 minutes at $4^{\circ} \mathrm{C}$ and the plasma aliquoted and stored at $80^{\circ} \mathrm{C}$ for later use.

\subsubsection{Tissue collection}

Samples were collected from routine abdominal surgery. A $3 \times 3 \mathrm{~cm}$ biopsy of hVAT was removed from the greater omentum via laproscopic scissors. No extra incision was required for the biopsy. Once removed, the tissue was sectioned into smaller pieces using a sterile blade and place into a $2 \mathrm{~mL}$ centrifuge tube containing $1.5 \mathrm{~mL}$

RNALater $^{\circledR}$ (Ambion Inc) to preserve tissue stability. Samples were transported to the Institute of Life Science 1 in Swansea University for storage at $4^{\circ} \mathrm{C}$ overnight before storage at $-20^{\circ} \mathrm{C}$ until required.

\subsubsection{RNA extraction}

RNA was extracted from hVAT samples. Samples were cut into pieces of $\leq 100 \mathrm{mg}$ and placed in a $2 \mathrm{~mL}$ homogeniser tube (Lysing Matrix D, MP Biomedicals). At room temperature, $1 \mathrm{~mL}$ of QIAzol lysis buffer was added, centrifuged at 4 meters per second for 20 seconds and then placed immediately on ice, this was repeated until 
the sample was completely homogenised. The lysate was transferred to a clean 1.5 $\mathrm{mL}$ tube and left for 5 minutes at room temperature. Chloroform (200 $\mu \mathrm{L})$ was added, shaken for 15 seconds and spun at 15,000 rpm $(12,000 \times \mathrm{g})$ for 15 minutes at $4^{\circ} \mathrm{C}$. This stage allows separation of the mixture into a lower red phenol/chloroform phase, a white interphase and a colourless upper aqueous phase which contains RNA. The upper aqueous layer was transferred into a clean $1.5 \mathrm{~mL}$ tube where 1 volume of $70 \%$ ethanol was added and immediately vortexed to mix. The next stage of the extraction used an RNAeasy mini kit $\left(\right.$ Qiagen $^{\mathrm{TM}}$ ) which contains all components and buffers, and is described in detail in section 2.1.8.

\subsubsection{DNA free treatment}

DNA free treatment was conducted as described in section 2.1.8.1.

\subsubsection{RNA quantification}

Extracted RNA was quantified as described in section 2.1.8.2.

\subsubsection{Analytical chemistries}

\subsubsection{Randox Daytona Plus}

The Randox Daytona Plus is a system for immunoturbidimetry and clinical chemistry analysis, providing rapid measurement of glucose, cholesterol, high density lipoprotein (HDL), albumin, creatinine and triglycerides in serum and plasma. Samples were run alongside quality control samples (Multisera Levels 2 and 3) and 
calibrated using saline and Calibration Serum Level 3 (Randox). The instrument uses direct photometry to measure a coloured endpoint to determine glucose concentration $(\mathrm{mmol} / \mathrm{L})$, total cholesterol $(\mathrm{mmol} / \mathrm{L}), \mathrm{HDL}$ cholesterol $(\mathrm{mmol} / \mathrm{L})$, triglycerides $(\mathrm{mmol} / \mathrm{L})$, creatinine $(\mu \mathrm{mol} / \mathrm{L})$ and albumin $(\mathrm{g} / \mathrm{L})$. Finally, LDL concentration (mmol/L) was calculated using values produced by the Randox Daytona Plus and utilizing the Friedewald equation, all concentrations are $\mathrm{mmol} / \mathrm{L}$ :

$$
L D L=\text { Total cholesterol }-H D L-\left(\frac{\text { Triglycerides }}{2.2}\right)
$$

\subsubsection{HbA1c analysis}

In humans, glucose binds to haemoglobin in the bloodstream which has a viability of 8-12 weeks. Therefore, measuring the glucose bound to haemoglobin gives an average blood glucose level for the past three months. Determination of the relative percentage of glycosylated haemoglobin in whole blood (HbA1c) was undertaken using the BioRad D-10 haemoglobin analyser, via high performance liquid chromatography (HPLC). Samples were pre-diluted (5 $\mu \mathrm{L}$ in $1500 \mu \mathrm{L}$ buffer) aspirated and introduced into the analytical flow path. The sample probe was rinsed between samples to reduce the risk of carryover. A programmed buffer gradient of increasing ionic strength delivered the sample to the analytical cartridge, where the haemoglobins are separated based upon their ionic interactions with the cartridge material. The separated haemoglobins were then passed through the filter photometer flow cell, where changes in the absorbance 
were measured at $415 \mathrm{~nm}$. HbA1c levels were recorded in both $\mathrm{mmol} / \mathrm{mol}$ and $\%$ units.

\subsubsection{Acyl-ghrelin mesurement}

\subsubsection{ELISAS}

Enzyme linked immunosorbent assay (ELISA) is a quantitative analytical method that shows antigen-antibody reactions through a colour change. This colour change is achieved via an enzyme-linked conjugate and substrate that when compared to a known concentration, i.e. a standard curve, can determine the concentration of specific molecules. There are two types of ELISAs; homogeneous and heterogeneous (Aydin, 2015). Homogeneous methods requires the enzyme to become inactivated upon antibody binding resulting in continuous antigen binding to the medium causing low sensitivity, however it is often used to quantify therapeutic substances in small quantities (O'Kennedy et al., 1990). The ELISAs used within the project are heterogeneous and require a wash stage to separate the bound antigen from the free antigen after antigen-antibody interaction. Heterogeneous ELISAs are based on 4 distinct methods; direct, indirect, sandwich and competitive (Aydin, 2015). Within this project all ELISAs were based on a sandwich method (Figure 2.4). 


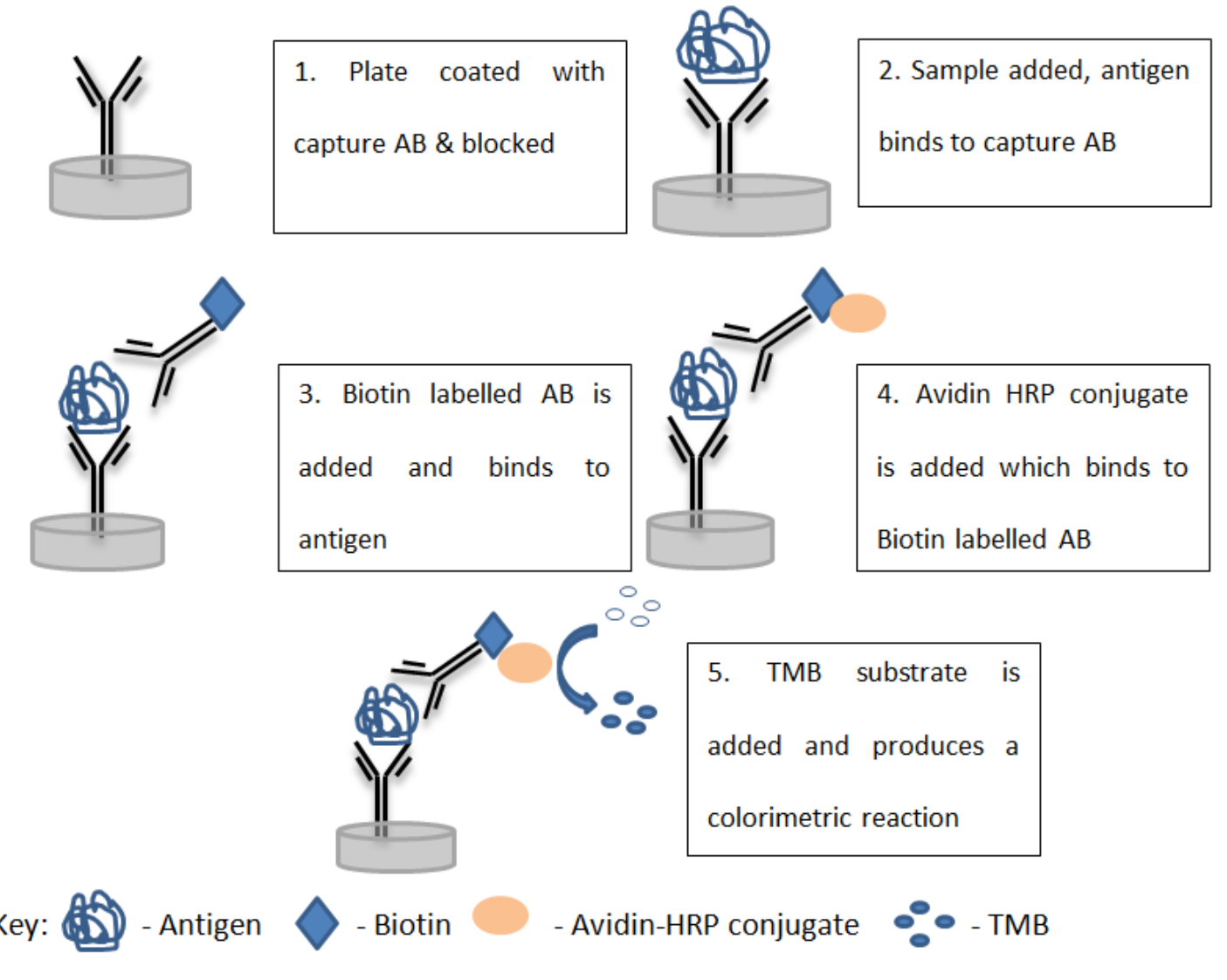

Figure 2.4. Key steps involved in a sandwich ELISA. (AB)-Antibody, (HRP)-Horseradish Peroxidase, (TMB)-3, 3', 5, 5'-tetramethylbenzidine.

\subsubsection{Acyl-ghrelin assay}

An acyl-ghrelin ELISA kit (Merck Millipore ${ }^{\mathrm{TM}}$ ) was used to determine fasting plasma acyl-ghrelin levels, the assay was completed as per manufacturer protocol using the reagents supplied within the kit. Assay is characterized with $100 \%$ specificity for human acyl-ghrelin and $0 \%$ desacyl-ghrelin specificity. The protocol required the addition of $20 \mu \mathrm{L}$ of matrix solution to the blank, standards and quality control wells. Then, $30 \mu \mathrm{L}$ of assay buffer was added to the blank and sample wells, with the addition of only $10 \mu \mathrm{L}$ of assay buffer to the standards and quality control wells. In duplicate, $20 \mu \mathrm{L}$ of standards were dispensed in ascending concentration order, 
followed by both quality controls and the unknown samples (AESBF treated plasma). Antibody solution mixture $(50 \mu \mathrm{L})$ of human active ghrelin detection and capture antibody was added to each well, and the plate incubated at room temperature, oscillating on a plate rocker for 2 hours. The plate was then washed 3 times, aspirated and $100 \mu \mathrm{L}$ of enzyme solution added per well for a further 30 minutes incubation oscillating at room temperature. The plate was then washed 6 times.

The colorimetric reaction was initiated with the addition of $100 \mu \mathrm{L}$ of substrate solution and the plate incubated for 5-20 minutes dependent on the rate of colour change. A blue colouration is formed in the wells of the standards, with intensity proportional to increasing concentrations of acyl-ghrelin. Stop solution (100 $\mu \mathrm{L})$ was added causing the blue colouration to turn yellow. Absorbance was read at $450 \mathrm{~nm}$ and corrected at $590 \mathrm{~nm}$ in a plate reader within 5 minutes of addition of stop solution. Acyl-ghrelin concentration was determined via comparison of the absorbance of unknown samples against the standard curve.

\subsubsection{Oxidative stress measurement}

Plasma total antioxidant status (TAOS) is based on the method previously described by Sampson et al, 2002 and Stephens et al, 2009. Laights photometric microassay allows the determination of TAOS values via its capability to inhibit the peroxidase-

mediated formation of the 2,2-azino-bis-3-ethylbensthiazoline-6-sulfonic acid (ABTS+) radical. Due to it being inversely related to oxidative stress, the inhibition 
of ABTS+ formation is proportional to the samples antioxidant capacity and therefore a low level of oxidative stress will result in a high TAOS value. Hydrogen peroxide acts as a free radical donor that causes the formation of ABTS+ radicals, thus producing a colorimetric change. Phosphate buffer saline (PBS) is used as a control as it contains no antioxidant molecules allowing the reaction to proceed to completion. Upon the addition of a plasma sample, the reaction is retarded from going to completion and the degree of inhibition is dependent upon the level of antioxidants within the sample. The percentage inhibition of the reaction is represented by the difference in absorbance divided by the control absorbance:

$$
\text { TAOS } \%=\frac{(\text { Control abs }- \text { sample abs })}{\text { Control abs }} \times 100
$$

The protocol required the addition of $2.5 \mu \mathrm{L}$ of plasma or PBS (control) to a clear 96-well ELISA plate in triplicate. Then $20 \mu \mathrm{L}$ ABTS (20 mmol/L), $20 \mu \mathrm{L}$ HRP (30 $\mathrm{mU} / \mathrm{mL}$ ) and $40 \mu \mathrm{L}$ PBS was added to each well, the reaction was initiated by the addition of $20 \mu \mathrm{L}$ hydrogen peroxide $(0.1 \mathrm{mmol} / \mathrm{L})$. The plate was incubated at $37^{\circ} \mathrm{C}$ for 12 minutes and read at $405 \mathrm{~nm}$ on a Crocodile ELISA miniWorkstation (TitertekBerthold $\left.^{\mathrm{TM}}\right)$.

\subsection{PCR}

Polymerase chain reaction (PCR) is a technique that is based on DNA polymerase's ability to synthesise new DNA strands, thus allowing for the amplification of a specific region of template sequence. The most commonly used DNA polymerase 
and one used in this methodology is Taq polymerase (from Thermus aquaticus). Standard PCR works on the principle of three main stages where double stranded DNA is heat denatured, specific primers align to the single DNA strands and DNA polymerase extends the primers resulting in two copies of the original DNA. This amplification cycle consisting of denaturation, annealing and elongation occurs at specific temperatures for multiple repeats (20-40 cycles) (Figure 2.5) which are optimised for the primer set to ensure a maximum product yield.

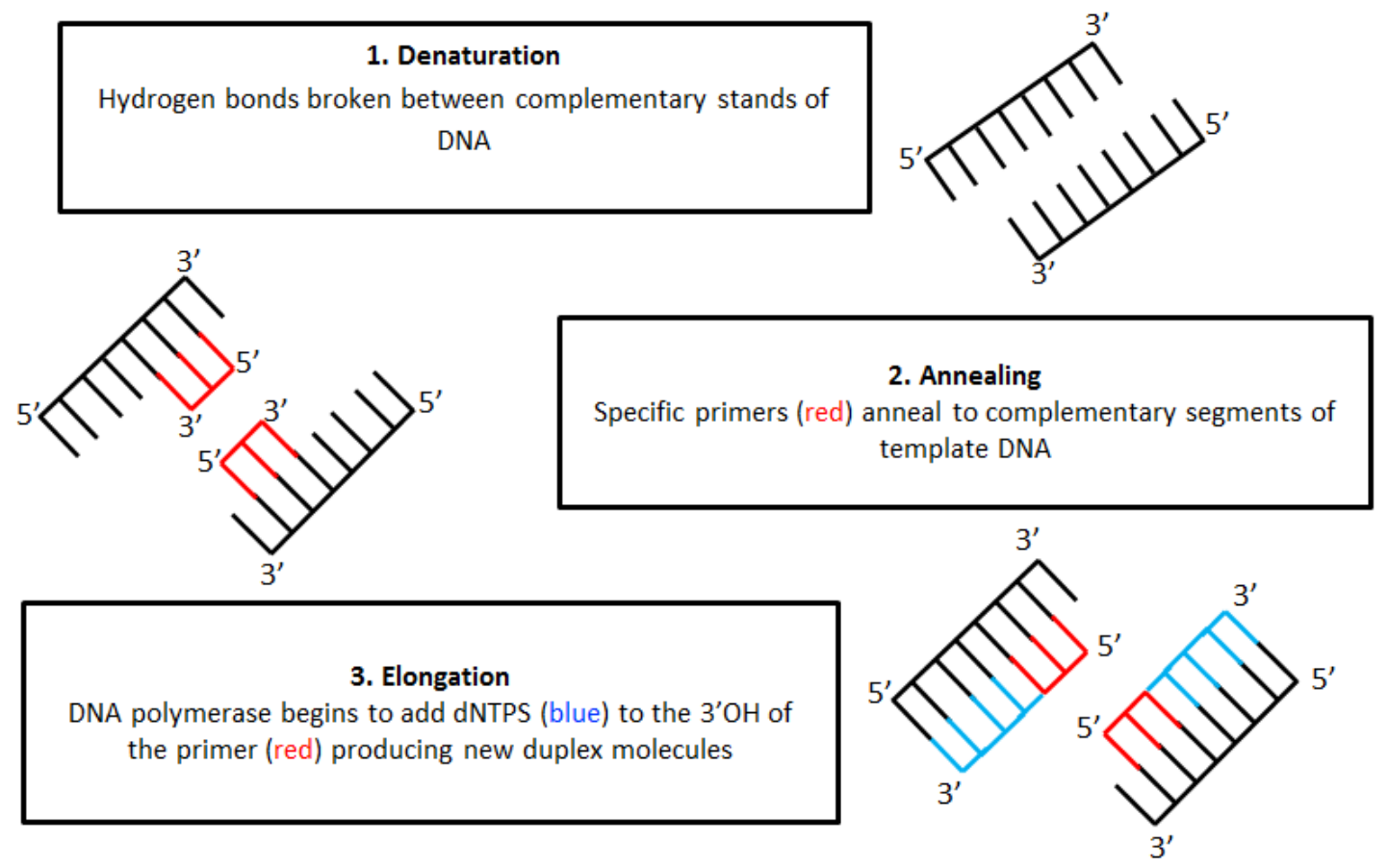

Figure 2.5. Polymerase Chain Reaction (PCR), three main stages and description. 


\subsubsection{Reverse transcription}

As previously mentioned, PCR utilises Taq polymerase which is unable to use RNA as a starting material, therefore the initial step of RT-PCR is to convert mRNA into a complementary strand of DNA (cDNA) using the enzyme reverse transcriptase. Reverse transcriptase is an RNA-dependent DNA polymerase that generates cDNA via the use of mRNA as a template in the presence of either oligo-dT or random decamer primers. Oligo-dT primers work by annealing to mRNA poly A tails, thus allowing for a reduction in background due to specific reverse transcription of mRNA. Contrary to this are random decamers, which consist of a pool of 6-10 nucleotides with all base combinations present, this method enables the reverse transcription of mRNA molecules too large for oligo dTs, however, this can increase background due to nonspecific annealing of RNA. The genes of interest within this study contained a final intron-exon boundary at various $\mathrm{Kb}$ distance upstream from the polyA tail. Therefore to ensure efficient reverse transcription of mRNA each specific primer required the use of either oligo dTs, random decamers or a combination of the two (see Table 2.4). 


\begin{tabular}{|c|c|}
\hline Primer Set & RT Primers \\
\hline$\beta$-actin & $50: 50$ mix \\
\hline PPARY & 50:50 mix \\
\hline ABCG1 & Oligo dTs \\
\hline LXR $\beta$ & Oligo dTs \\
\hline Ghrelin & Oligo dTs \\
\hline LYPLA1 & Random Decamers \\
\hline mBOAT4 & Oligo dTs \\
\hline SREBF1 & Oligo dTs \\
\hline GHSR-1 $\alpha$ & Oligos dTs \\
\hline
\end{tabular}

Table 2.4. Reverse transcription primers used for cDNA synthesis for each specific primer set.

\subsubsection{2 cDNA synthesis}

The mRNA within total RNA extracted from tissue and cells underwent reverse transcription using an Ambion ${ }^{\mathrm{TM}}$ RETROscript kit following the manufacturer's protocol. A master mix for the desired amount of reaction was prepared containing the following components, ensuring each reaction compiled of the exact same quantities: 
$2 \mu \mathrm{L}$ random decamers [50 $\mu \mathrm{M}$ ] OR $2 \mu \mathrm{L}$ oligo-dTs [50 $\mu \mathrm{M}]$ OR

$1 \mu \mathrm{L}$ random decamers \& $1 \mu \mathrm{L}$ oligo-dTs

$2 \mu \mathrm{L} 10 \times \mathrm{RT}$ Buffer

$4 \mu \mathrm{L}$ dNTP mix [2.5 mM each dNTP]

$1 \mu \mathrm{L}$ MMLV reverse transcriptase $[100 \mathrm{U} / \mu \mathrm{L}]$

The master mix was vortexted and centrifuged and $9 \mu \mathrm{L}$ placed into a $0.2 \mathrm{~mL}$ centrifuge tube. $1 \mu \mathrm{g}$ of total RNA was then added and brought to a final volume of $20 \mu \mathrm{L}$ using RNase free water. The final reaction was mixed, briefly spun and incubated at $44^{\circ} \mathrm{C}$ for 1 hour then at $92^{\circ} \mathrm{C}$ for a further 20 minutes to inactivate the reverse transcriptase, and stored at $-20^{\circ} \mathrm{C}$ for future use.

\subsubsection{End point RT-PCR}

End point RT-PCR utilises the basic principle of standard PCR to confirm the expression of key genes related to this project. Standard PCR undergoes 3 phases. Initially the reaction has an exponential phase where the exact doubling of product is accumulating at every cycle. This leads into a linear phase, which is due to the reaction slowing and components being consumed. Finally, the PCR enters a plateau phase, where the reaction stops and no more PCR product is generated. At this point the PCR product can be visualised on a gel, known as end point detection. Visualising the PCR product at this point does not allow for the quantitation of mRNA expression levels, however it does allow for a rapid and cost-effective method for the confirmation of the presence or absence of key genes within sample types. 


\subsubsection{Reaction}

All reagents were sourced from Promega ${ }^{\mathrm{TM}}$, the following was used for each end point PCR sample and all reactions were prepared within a bench top PCR hood cleaned with $70 \%$ ethanol and UV irradiation for 15 minutes prior to use. The cycling conditions were performed within a BioRad CFX Connect and specific cyclic conditions for each primer set are described in Appendix 2.

$10 \mu \mathrm{L} 5 \mathrm{X}$ Colourless Go ${ }^{\circledR}$ Taq Flexi Buffer

$3 \mu \mathrm{L} \mathrm{MgCl} 2[25 \mathrm{mM}]$

$1 \mu \mathrm{L} d \mathrm{NTP} \operatorname{mix}[40 \mathrm{mM}]$

$0.5 \mu \mathrm{L} \mathrm{Go}{ }^{\circledast} T a q \mathrm{G} 2$ Flexi DNA Polymerase [5 $\left.\mathrm{U} / \mu \mathrm{L}\right]$

$1 \mu \mathrm{L}$ Forward Primer [15 pmol]

$1 \mu \mathrm{L}$ Reverse Primer [15 pmol]

$30.5 \mu$ L Deionised, sterile $\mathrm{H}_{2} \mathrm{O}$

$3 \mu \mathrm{L}$ Sample cDNA or negative control $\left(\mathrm{H}_{2} \mathrm{O}\right)$

\subsubsection{Gel electrophoresis}

PCR products were visualised on a $12 \%$ polyacrylamide gel using the following reagents:

Acrylamide:Bisacrylamide (37.5:1) stock solution, containing $30 \% \mathrm{w} / \mathrm{v}$ acrylamide (Severn Biotech Ltd)

10x Tris/Borate/EDTA Buffer (Invitrogen)

TEMED ( $\mathrm{N}_{1} \mathrm{~N}_{1} \mathrm{~N}_{1} \mathrm{~N}_{1}$-tetramethylethylene diamine) (Sigma-Aldrich) 
10\% Ammonium persulphate (Sigma-Aldrich)

$12 \%$ polyacrylamide gels were made by diluting $16 \mathrm{~mL}$ Acrylamide:Bisacrylamide solution and $4.5 \mathrm{~mL}$ 10X TBE buffer in $24 \mathrm{~mL}$ distilled water. Polymerisation of the acrylamide was initiated by the addition of $220 \mu \mathrm{L}$ of $10 \%$ APS and $45 \mu \mathrm{L}$ of TEMED. The solution was then placed into four BioRad caskets and a 15-tooth comb inserted. The gels were set at room temperature for approximately 30 minutes then wrapped and stored at $4^{\circ} \mathrm{C}$ for future use.

Gels were placed into vertical gel cases and filled with 1x TBE buffer. $5 \mu \mathrm{L}$ PCR product was mixed with $2 \mu \mathrm{L}$ gel loading buffer ( $50 \% \mathrm{v} / \mathrm{v}$ glycerol, $10 \% \mathrm{v} / \mathrm{v} 0.1 \mathrm{M}$ EDTA, $1 \% \mathrm{v} / \mathrm{v}$ bromophenol blue, 1\% SDS) (Sigma-Aldrich), with $5 \mu \mathrm{L}$ of mixture pipetted into the loading well. Each gel also contained a reference 100 base pair (bp) ladder, $5 \mu \mathrm{L}$ of a premixed ladder was loaded into the first well of every gel. The premixed ladder contained; $1 \mu \mathrm{L} 100 \mathrm{bp}$ ladder (Promega), $2 \mu \mathrm{L}$ gel loading buffer and $5 \mu \mathrm{L}$ distilled water.

Gels were visualised using silver staining, which required the gel being placed in silver nitrate solution ( $1 \mathrm{~g} \mathrm{AgNO}_{3}$ (Sigma-Aldrich) in $1 \mathrm{~L} \mathrm{H}_{2} \mathrm{O}$ ) under gentle agitation for 10 minutes. Silver nitrate solution was decanted off and residual solution washed off with distilled water. The gel was then placed in sodium hydroxide solution (13.5g NaOH (Sigma-Aldrich) in $900 \mathrm{~mL} \mathrm{H}_{2} \mathrm{O}$ and $3.6 \mathrm{~mL}$ formaldehyde (Sigma-Aldrich)) for a further 10 minutes under gentle agitation. Gels were imaged using a white tray on a BioRad EZ Imager and analysed using BioRad Image Tool software. 


\subsubsection{Quantitative Real time PCR}

Gene expression profiling analysis via quantitative real time PCR (qReal Time PCR) allows for the reliable detection and quantification of nucleic acids during the exponential phase of a PCR. qReal Time PCR works on the same amplification principle of PCR, however the experimental design is based on the detection and quantification of fluorescence emitted by a reporter molecule in real time. RNA transcripts are reverse transcribed into cDNA first then used in the qReal Time PCR. The accumulation of PCR product generated with each cycle emits an increased fluorescence that once it has passes a certain threshold, the cycle in which this occurs can be quantified ( $\mathrm{Ct}$ value). The $\mathrm{Ct}$ value is directly correlated to the initial amount of target template, allowing for the quantification of gene expression. Reporter molecules that are most commonly used are DNA binding agents (SYBRgreen dye) and hydrolysis probes (Taqman).

\subsubsection{SYBRgreen 1}

Throughout the analysis, SYBRgreen I (BioRad ${ }^{T M}$ ) was utilised as a reporter molecule. SYBRgreen I is an intercalating dye that binds to the double stranded DNA, emitting fluorescence that correlates with DNA amplification. The advantage of using SYBRgreen I over complex probes is that it is an intercalating dye which offers a simple and cost-effective approach. However, due to its ability to bind to any double stranded DNA it is susceptible to error. Primer optimisation is key to decreasing the risk of non-specific primer dimer formation. 


\subsubsection{Real time PCR primer design}

Real Time PCR primers were designed using the NCBI tool Primer BLAST. All primers were designed using the RNA gene sequence to be approximately 20 nucleotides in length, with an annealing temperature of approximately $60^{\circ} \mathrm{C}$. The tool returned several primer pairs for comparison and the primer pair selected was based on a GC content of around $50-60 \%$ to ensure product stability and avoiding selfcompatibility, reducing the possibility of primer dimer. The primer sequences were then located within the gene sequence to ensure they crossed an exon-exon boundary. The primers were designed to produce an amplicon of 150-250 bp in size from GHRL, PPARY, ABCG1, LXRB and LYPLA1 as target genes, and B-actin as a housekeeper gene.

Oligonucleotides were synthesised by Eurofins MWG Operons (Germany) and shipped desalted and dehydrated. Upon arrival, they were re-hydrated with the stated volume of filter sterilised water to produce a concentration of $100 \mathrm{pmol} / \mu \mathrm{L}$, then diluted to $15 \mathrm{pmol} / \mu \mathrm{L}$, aliquoted and stored at $-20^{\circ} \mathrm{C}$.

Due to the quantity of time required to design and optimise, additional primers for SREBF1 and mBOAT4 that proved difficult to optimise in-house, were designed and validated by Primerdesign ${ }^{\mathrm{TM}}$. The company was supplied with the target genes of interest and returned the designed and optimised primer sets. The product came with a guaranteed priming specificity and efficiency of $>90 \%$. The lyophilised primer mix was resuspended in $660 \mu \mathrm{L}$ filter sterilised water to achieve a $300 \mathrm{nM}$ working concentration, aliquoted and stored at $-20^{\circ} \mathrm{C}$. All primer sequences are listed in Appendix 2. 


\subsubsection{Real Time PCR reaction}

All reactions were prepared within a bench top PCR hood that was cleaned with $70 \%$ ethanol and UV irradiated for 15 minutes prior to use. The cycling conditions were performed within a BioRad CFX Connect and each primer set required a distinct and optimised cycling condition (Appendix 2). However, each Real Time PCR reaction conducted was uniform, consisting of the following reagents:

$12.5 \mu \mathrm{L}$ SYBRgreen I

$1 \mu \mathrm{L}$ Forward Primer [15 pmol]

$1 \mu \mathrm{L}$ Reverse Primer [15 pmol]

$5 \mu \mathrm{L}$ cDNA

$5.5 \mu \mathrm{L}$ Deionised, sterile $\mathrm{H}_{2} \mathrm{O}$

\subsubsection{Analysis of Real Time PCR data; $2^{-\Delta \Delta C T}$ Method}

Relative quantitation was used to determine differences in expression levels of the specific target genes between different samples. This generates a fold change output that represents the change in expression of a particular gene either over time or in varying treatments. In order to obtain accurate relative quantitation of an mRNA target, the expression level of an endogenous control (housekeeper) $\beta$ actin were also measured (Livak and Schmittgen, 2001).

To ensure that the comparative $\mathrm{Ct}$ method or $2^{-\Delta \Delta C T}$ method could be used for data analysis a validation experiment was conducted. In order to determine a 
fold change value, the raw $\mathrm{Ct}$ values were analysed using a 3 step process. Firstly, it was required make the target $\mathrm{Ct}$ value relative to an endogenous housekeeper:

$$
\Delta \mathrm{Ct}=\mathrm{Ct}_{\text {(target) }}-\mathrm{Ct}_{\text {(housekeeper) }}
$$

Then, determine the magnitude of the fold change in respect to a calibrator group i.e. treated versus an untreated sample:

$$
\Delta \Delta \mathrm{Ct}=\Delta \mathrm{Ct}_{\text {(test sample) }}-\Delta \mathrm{Ct}_{\text {(calibrator sample) }}
$$

Finally, the $\Delta \Delta$ Ct must be log transformed:

$$
2^{-\Delta \Delta C t}
$$

A positive $\Delta \Delta$ Ct will produce a fold change of $<1$. In order to establish the magnitude of fold change the value is expressed as either a fraction or 1 over. For example, $2^{-\Delta \Delta C t}=2^{-(2.0)}=0.25$, fold change can either be expressed as $1 / 4$ or as $1 / 0.25$ to produce a 4-fold down regulation.

\subsubsection{PCR controls and validations}

\subsubsection{PCR controls}

The controls within PCR consisted of a no-template control (NTC) and no-reverse transcription control (NRTC). NTC contains all the Real Time PCR reagents without the cDNA template, allowing the detection of any contamination within the 
mastermix. NRTC allows for the detection of any contamination within the reverse transcription step since it contains all reagents other than template RNA.

\subsubsection{Primer validation}

To ensure a comparative method could be used within data analysis, all primers had to have the same standard efficiency. If the primer efficiencies were approximately equal then there would be limited variation in the $\Delta C T$ value within the template dilution (Schmittgen and Livak, 2008). To ensure this was correct for all primers, cDNA was generated from 3 separate RNA samples of hVAT (Section 2.2.5) and a template dilution series over 3 points produced. Real Time PCR was performed for each sample using all generated primers and $\Delta C T$ values established. Data was plotted to produce a semi-regression plot, where primers were accepted on the basis of slope value of $<0.1$. Validation plots for each primer set can be found in Appendix 3.

\subsubsection{Real Time PCR Validation}

In order to determine the assay's reproducibility, both inter- and intra-assay variation for Real Time PCR data generated were assessed from each primer set used. Intra-assay variation was determined by performing 5 assays (each in triplicate) for each primer set, on 3 different RNA samples (from hVAT) on a single plate within the same Real Time PCR run. Inter-assay variation examined the variation produced within a sample for each primer set on different Real Time PCR 
runs. Therefore, 3 samples sourced from hVAT were run in triplicate for each primer set on 5 different assays over the course of 10 days. Intra- and inter-assay coefficient of variances are given in Table 2.5 (raw data found in Appendix 4).

\begin{tabular}{|c|c|c|}
\hline Primer Set & Intra-variation (CV\%) & Inter-variation (CV\%) \\
\hline B-actin & $0.66-0.84$ & $1.06-1.47$ \\
\hline PPARY & $1.10-1.85$ & $1.60-2.02$ \\
\hline ABCG1 & $0.74-1.10$ & $1.05-1.24$ \\
\hline LXRB & $0.83-1.38$ & $1.76-2.34$ \\
\hline GHRL & $0.88-1.39$ & $1.22-1.63$ \\
\hline LYPLA1 & $0.42-0.84$ & $0.74-1.01$ \\
\hline mBOAT4 & $0.93-1.10$ & $1.34-2.01$ \\
\hline SREBF1 & $1.02-1.26$ & $0.83-1.42$ \\
\hline
\end{tabular}

Table 2.5. Intra- and inter-variations for Real Time PCR for all primer sets. 


\subsection{Adipokine ELISAs}

Tumor necrosis factor alpha (TNF $\alpha$ ), interlukin-6 and interleukin-10 (IL-6 and IL-10) were measured using sandwich ELISA assays purchased from R\&D systems ${ }^{T M}$, as described in Section 2.2.7.1. The ELISA for each adipokine used reagents within the kit and in accordance with the manufacturer's guidance. Within this project, both SGBS cell culture supernatants and human plasma collected time of procedure were needed. Each kit was brought to room temperature before use and standards made from a supplied lyophilised stock. All samples were thawed and centrifuged at $4000 \mathrm{rpm}(1,431 \mathrm{xg})$ for 5 minutes before assaying and plated onto a standard plate layout (Figure 2.6).

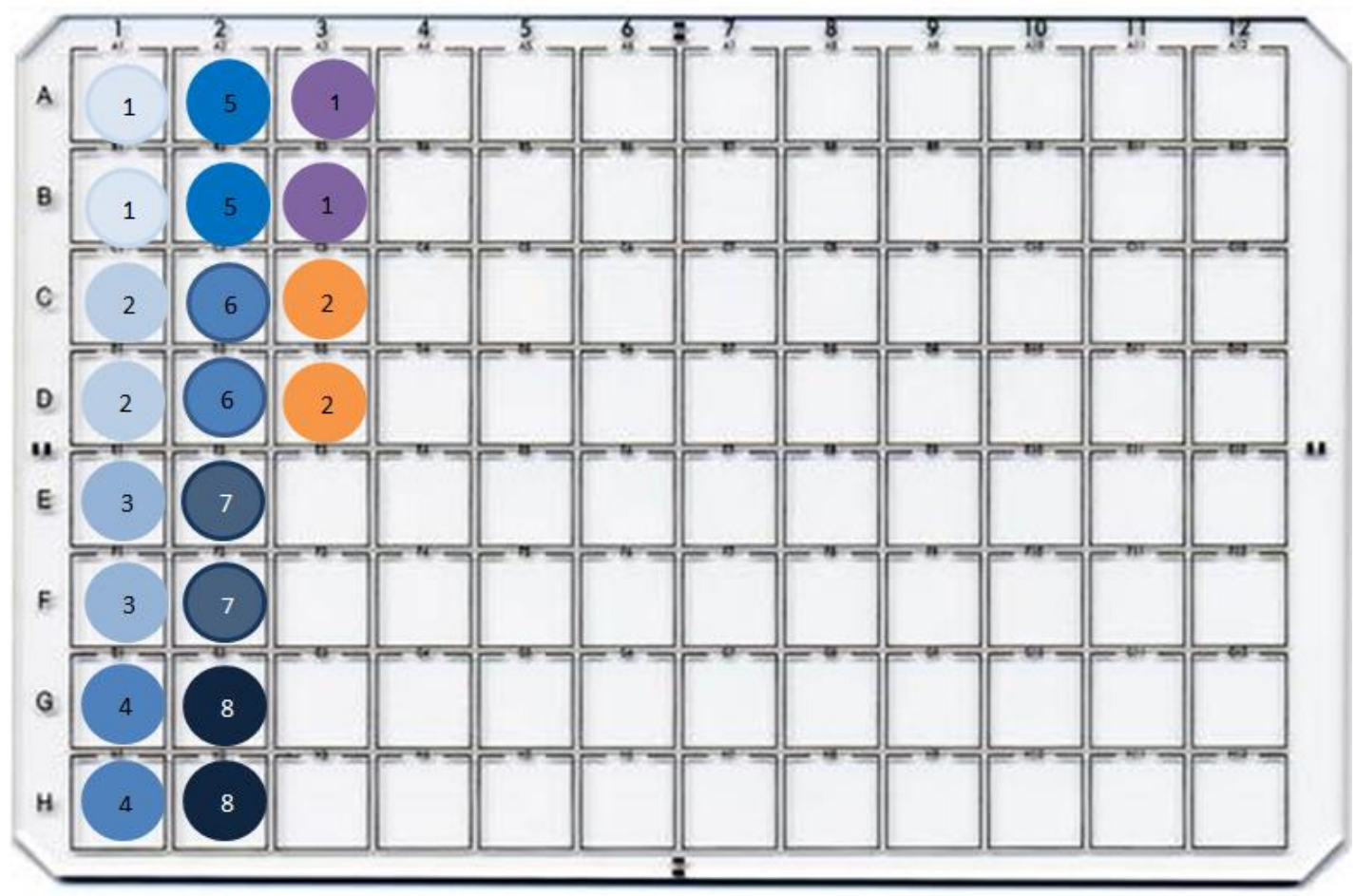

Figure 2.6. Plate layout for adipokine ELISAs. The 8 standards in duplicate were placed in ascending concentration (1-8, blue gradient). Standard curve is followed by unknown samples in duplicate (1 \& 2, purple and orange). 


\subsubsection{TNF $\alpha$}

The protocol required the addition of $50 \mu \mathrm{L}$ of assay diluent to each well, followed by $200 \mu \mathrm{L}$ of known standards, quality controls and unknown samples in duplicate. After a 2 hour incubation at room temperature, the plate was washed and aspirated. Subsequently $200 \mu \mathrm{L}$ of TNF $\alpha$ conjugate was added and incubated at room temperature for either 1 hour for cell culture supernatant or 2 hours for human plasma samples. The plates underwent a final wash cycle before the initiation of the colourimetric reaction as described in Section 2.4.4.

\subsubsection{IL-6}

The IL- 6 assay protocol required the addition of $100 \mu \mathrm{L}$ assay diluent, then $100 \mu \mathrm{L}$ of standards and unknown samples to each well in accordance with standard plate layout (Figure 2.6). After a 2 hour incubation at room temperature, the plate was washed and aspirated and $200 \mu \mathrm{L}$ of human IL- 6 conjugate added to each well for a further 2 hour room temperature incubation. The microplate then underwent a final wash cycle before initiating the colourimetric reaction as described in Section 2.4.4.

\subsubsection{IL-10}

The IL-10 procedure required a 10 -fold dilution of cell supernatant samples in specific calibrator diluent before running, however plasma samples were run neat. 
For plasma samples the microplate required the addition of $50 \mu \mathrm{L}$ of assay diluent before loading $200 \mu \mathrm{L}$ of standards or samples per well, whilst $200 \mu \mathrm{L}$ diluted cell supernatant samples were added directly to the plate. Both sample types required

a 2 hour room temperature incubation. The plate was then washed and aspirated and $200 \mu \mathrm{L}$ of human IL-10 conjugate added. Sample types required different incubation times at room temperature, with cell supernatants requiring a 1 hour incubation and plasma samples requiring a 2 hour incubation. The plate underwent a final wash cycle before initiating the colourimetric reaction as described in Section 2.4.4.

\subsubsection{Colourimetric reaction}

All assays underwent the same protocol to produce a colourimetric reaction. The colourimetric reaction was initiated with the addition of $200 \mu \mathrm{L}$ of substrate solution and incubated for 20 minutes dependent on the rate of colour change. 50 $\mu \mathrm{L}$ of stop solution was added causing the blue colouration to turn yellow after acidification. Absorbance was read at $450 \mathrm{~nm}$ and corrected at $590 \mathrm{~nm}$ in a plate reader within 5 minutes of addition of stop solution. Adipokine concentration was determined via comparison of the absorbance of unknown samples against the known concentration of the standards. 


\subsection{Statistical analysis}

All statistical analysis of raw data was conducted using SPSS version 22 and figures were constructed using Microsoft Excel. A p-value of $<0.05$ was deemed statistically significant for all tests.

\subsubsection{Categorical data}

Categorical data that was represented by numbers $(n)$ and percentage (\%) were analysed using pearson chi squared test.

\subsubsection{Continuous data}

The continuous data throughout this thesis is summarized by mean ( \pm standard deviation (SD)), when the data were normally distributed and by median and interquartile range (IQR) if not normally distributed. Normality was checked using Kolmogorov-Smirnov test and visualised on q-q plots. The student t-test was used to compare the mean of two groups and analysis of variance (ANOVA) to compare the mean of more than two groups for normally distributed data. Alternatively, non-normally distributed data was analysed using Whitney $U$ and Kruskal Wallis tests. For the analysis of association between two continuous variables within normally distributed data, a Pearson correlation coefficient test was conducted, where a positive or negative correlation spanned from -1 to 1 , with 0 indicating no correlation. Within non-normally distributed data, a Spearman's Rho test was conducted. 
Chapter 3

\section{Effect of acyl-ghrelin in vitro on mature adipocytes in hyper- and normoglycaemic environments}




\subsection{Introduction}

Adipose tissue provides a vast store of energy which contributes to whole body homeostasis. In mammals, long term energy is stored within the lipid droplets of adipocytes, with over $90 \%$ stored in the form of triglycerides (Shen et al., 2003). Obesity results in the enlargement of adipose tissue to store the excess energy intake. The way in which adipose tissue facilitates this enlargement is dependent upon both diet and genetics; and their interaction together (Jo et al., 2009).

\subsubsection{Lipid droplet storage in mature adipocytes}

Mature adipocytes contain multiple lipid droplets, ranging between 25 and $150 \mu \mathrm{m}$ in diameter. In a fed state, the human body will store excess energy via active insulin-dependent GLUT4, allowing for the uptake of glucose from the bloodstream into adipocytes. Intracellular adipocyte esterification of FFA (lipogenesis) occurs when the chylomicrons from the intestine esterify glycerol-3-phosphate, the product of glycolysis, to form lipid droplets of triglycerides. Adipocyte hydrolysis of triglycerides (lipolysis) produces adipose tissue's major secretory product, FFA (Wood and Trayhurn, 2006). If the rate of lipogenesis exceeds that of lipolysis then adipocyte cells either adapt via expansion in lipid droplet size or cellular lipid area (hypertrophy), or recruit an increased number of adipocytes (hyperplasia). Hypertrophy occurs prior to hyperplasia and this is the initial cellular response to allow for storage of additional triglycerides in the progression of obesity. 


\subsubsection{Hypertrophy-induced inflammation and Type 2 diabetes}

An increase in adipocyte size due to an inability to proliferate is deemed to have pathological consequences for both metabolic and immune functions (Bays et al., 2008). Human studies indicate that in individuals with obesity and T2D there is a decline in the expression of adipogenic genes, resulting in the storage of energy predominantly through lipogenesis and adipocyte hypertrophy, rather than the promotion of hyperplasia (Bays et al., 2008, Dubois et al., 1979, Dubois et al., 2006). Adipose tissue that contains hypertrophic adipocytes secrete pro-inflammatory proteins that result in low-grade inflammation and insulin resistance in obese individuals, which are implicated in the onset of T2D (Figure 3.1) (Kennedy et al., 2009).

Pro-inflammatory adipokines promote insulin resistance via the suppression of insulin signalling and alterations in glucose homeostasis. Saturated FFA, gutderived bacteria, LPS and pro-inflammatory adipokines have been shown to activate key inhibitory molecules within insulin signalling such as $\mathrm{NF}_{\mathrm{K}} \mathrm{B}$, suppressor of cytokine signalling (SOCS) and JNK (Shi et al., 2006, Ghoshal et al., 2009, Kwon and Pessin, 2013). 

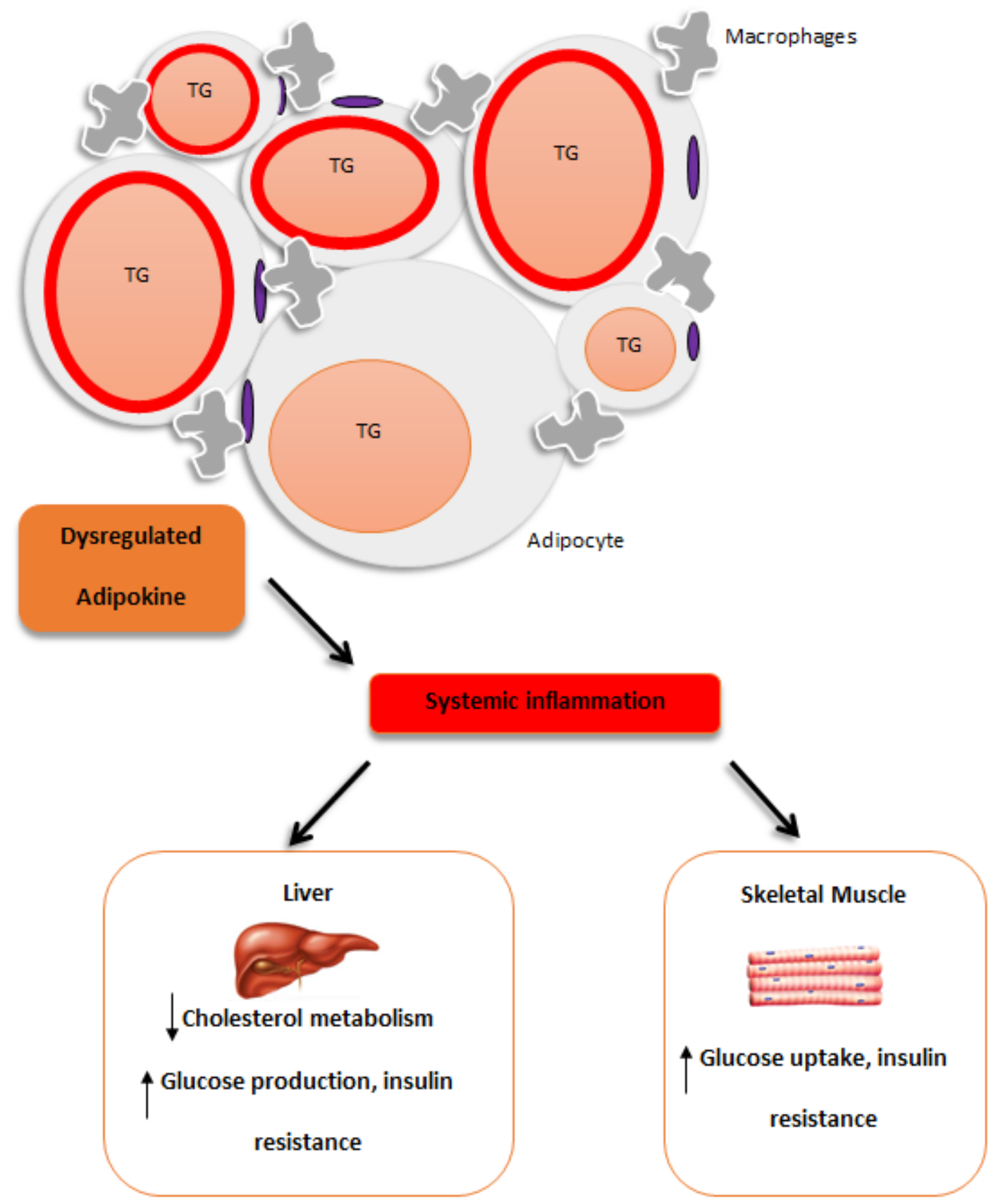

Figure 3.1. Overview of the downstream effects of hypertrophic adipocytes in obesity. The Red outer circles within adipocytes indicate hypertrophic stress, purple segments represents the nucleus and triglycerides are indicated by TG. Dysregulated adipokine release produces systemic inflammation and alters lipid and glucose metabolism in the liver and skeletal muscle. 


\subsubsection{Markers of inflammation}

Controversy exists as to which are the most appropriate adipokines to measure in relation to adipose tissue and its relationship to the metabolic state. Most notably described in association with the metabolic state and adipocyte size are the proinflammatory factors TNF $\alpha$ and IL-6 (Ronti et al., 2006), and the anti-inflammatory factor IL-10 (Esposito et al., 2003).

\subsubsection{TNFa}

TNF $\alpha$ was the first recorded adipokine to demonstrated an association between obesity, inflammation and T2D. This finding was underpinned by studies demonstrating that TNF $\alpha$ is highly expressed in obesity and is implicated in the pathogenesis of insulin resistance in adipose tissue (Stanley et al., 2011, Hotamisligil et al., 1995, Hotamisligil et al., 1993). TNFa impairs insulin action through inhibition of IRS signalling via the activation of serine kinases (Kwon and Pessin, 2013). Furthermore, TNF $\alpha$ promotes the expression of inflammatory cytokines and chemokines within adipocytes (Rotter et al., 2003).

\subsubsection{IL-6}

IL-6 is a pro-inflammatory adipokine that is involved in obesity-related insulin resistance (Ouchi et al., 2011). IL-6 is secreted from WAT, with approximately equal gene expression produced by adipocytes and infiltrated macrophages (Wisse, 2004). IL-6 function in insulin resistance and glucose homeostasis is influenced by tissue type and metabolic state. Systemic increase in IL-6 is has a detrimental effect by increasing insulin resistance (Makki et al., 2013). Within adipose tissue and the 
liver, IL-6 results in increased insulin resistance through impairing insulin-induced insulin receptor and IRS action via the up regulation of SOCS3 (Rotter et al., 2003, Senn et al., 2003)

\subsubsection{IL-10}

The anti-inflammatory adipokine IL-10 has a role in the attenuation of the immune response triggered by the action of pro-inflammatory markers such as IL-6 and TNF $\alpha$ (Stenvinkel et al., 2005). IL-10 secretion has been described from both nonadipocyte and adipocytes fractions, with adipocyte size being negatively associated with IL-10 release (Skurk et al., 2007). Clinical studies have shown an association between reduced levels of IL-10 in obesity and the metabolic syndrome (Esposito et al., 2003, Skurk et al., 2007). 


\subsection{Aims}

Within this chapter I predict that findings will provide evidence for a detrimental role for acyl-ghrelin in the regulation of key genes within the lipid retention pathway, resulting in an increase in lipid retention area and in turn the promotion of inflammatory marker levels. To provide insight into my hypothesis, this chapter aimed to:-

1. Determine the effect of acyl-ghrelin exposure on human mature adipocytes in different glycaemic environments

2. Establish the effect of chronic hyperglycaemia and acyl-ghrelin exposure on lipid retention

3. Establish the effect of chronic hyperglycaemia and acyl-ghrelin exposure on inflammation 


\subsection{Methods}

\subsubsection{SGBS cell treatment}

SGBS cells were cultured and differentiated as described in Sections 2.1.1 to 2.1.4. Cell dosage concentrations with acyl-ghrelin were based on previous published literature. Due to the novel aspect of the experiment, whereby acyl-ghrelin treatment of human mature adipocytes has not been previously recorded, therefore, dosage was based on acyl-ghrelin treated human macrophage and endothelial cell lines (Asakawa et al., 2003, Zhu et al., 2014). Chronic hyperglycaemic environments were introduced 48 hours prior to cell dosage with acyl-ghrelin or PBS vehicle. As previously mentioned in Section 2.1.5, glucose levels were determined by the physiological ranges present within individuals with T2D (25 $\mathrm{mM}$ ) and healthy controls (5.5 mM). To examine acyl-ghrelin mediated effects on the inflammatory state, cells underwent an immune challenge with the administration of $1 \mu \mathrm{g} / \mathrm{mL}$ LPS, with dosage determined via previously published data within similar experiments (Beynon et al., 2013, Waseem et al., 2008). LPS is an integral component of the outer membrane of gram negative bacteria that stimulates innate immunity, and is a potent tool in the examination and evaluation of anti-inflammatory agents. Cell supernatant measure of key inflammatory markers (TNF $\alpha$, IL-6 and IL-10) post exposure to LPS, acyl-ghrelin or a combination of LPS and acyl-ghrelin allows for the exploration of acyl-ghrelin as an inflammatory mediator. Oil red $\mathrm{O}$ staining and RNA extraction occurred at two time points; 17 and 34 hours. The time points depict a point of collection before a half and a full cell 
cycle respectively, which would normally consist of 38 hours. Treatment wells were run in duplicate on each 6-well plate, and each plate was run in triplicate.

\subsubsection{Validation of GHS-R1 $\alpha$}

To validate whether acyl-ghrelin presence could alter adipocyte function via the GHS-R1 $\alpha$, end point PCR was used to determine the presence of the acyl-ghrelin receptor (Section 2.3.2). All samples provided a positive result for the presence of GHSR-1 $\alpha$, with a product band at $118 \mathrm{bp}$, as shown below (Figure 3.2).

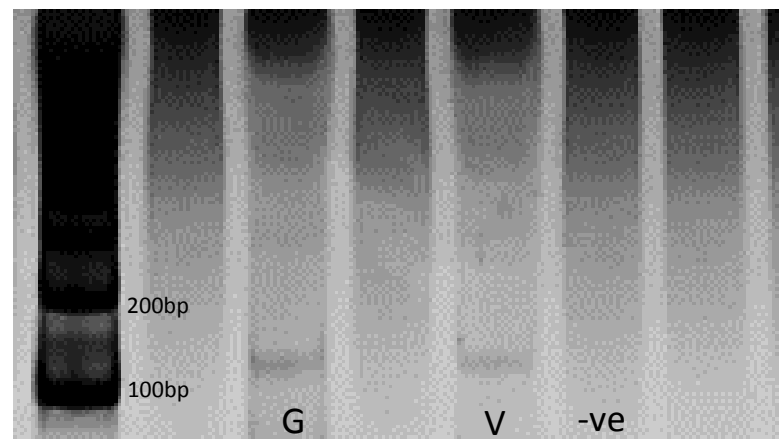

Figure 3.2. $12 \%$ polyacrylamide gel showing PCR products for GHSR1 $\alpha$ (118 bp) for RNA extracted at 17 hours from cells grown in a high glucose environment with acyl-ghrelin (G) or vehicle/PBS (V).

\subsubsection{Lipid accumulation measurements}

Lipid area was determined using Oil red $O$ staining as described in Section 2.1.6. Image analyses were conducted using ImageJ software (Version 2) and a published protocol for the quantification of lipid area (Mehlem et al., 2013). Each experimental well produced 3 magnifications of images in 5 fields, each field was predefined and set prior to cell seeding to remove experimental bias, with 20 cells at x50 magnification analysed to determine cell diameter and intracellular lipid 
area. This equates to 100 cells analysed per treatment, in duplicate. Cellular size measurements were recorded as followed;

- Cellular diameter- the average span of the cell across its longitudinal axis $(\mu \mathrm{m})$ within a treatment well

- Cellular area- the average intracellular lipid area for cells $\left(\mu \mathrm{m}^{2}\right)$ within a treatment well

- Global cellular diameter- the average span of the cell across its longitudinal axis $(\mu \mathrm{m})$ across all experiment wells

- Global cellular area- the average intracellular lipid area for cells $\left(\mu \mathrm{m}^{2}\right)$ across all experiment wells

\subsubsection{Gene expression measurements}

Gene expression levels were determined using Real Time PCR (Section 2.3) from RNA extracted from cells (Section 2.1.8). Each gene had a specific primer set and was subject to an optimised Real Time PCR cycling condition (Appendix 2). Results were analysed using the $2^{-\Delta \Delta C T}$ method as previously described (Section 2.3.4), thus allowing for the calculation of gene expression in a relative manner. The mean $\mathrm{Ct}$ for each gene within the treatment well was normalised against the threshold cycle data of the housekeeping gene ( $\beta$-actin). The normalised $\Delta \mathrm{Ct}$ data was used in further analysis to measure gene expression fold change across the treatment sets for each gene of interest. Fold change differences less than 0.5 were classified as down regulation of gene expression and $1 / x$ representing a true fold change. $A$ fold 
change difference of greater than 1.5 illustrated an up-regulation in gene expression (raw data shown in appendix 5).

\subsubsection{Inflammatory marker measurements}

Inflammatory markers (TNF $\alpha$, IL-6 and IL-10) were measured in LPS and acyl-ghrelin, separately and combined, treated cells, in both normo- and hyperglycaemic environments using specific ELISAs (Quantikine ${ }^{\circledR}, R \& D$ systems), as principal discussed in Section 2.4. Two aliquots of $1 \mathrm{~mL}$ cell supernatant were collected at each time point for each treatment duplicate and stored at $-80^{\circ} \mathrm{C}$ until analysed. Each ELISA consisted of an 8-point standard curve from which protein concentration was determined via sample absorbance plotting and a positive quality control sample (see plate layout (Figure 3.3)). Sample values with a coefficient of variance of less than $15 \%$ between duplicates were accepted.

\subsubsection{Data analysis}

Raw data retrieved from this chapter was treated as described in Section 2.5 using SPSS $^{\mathrm{TM}}$ Version 22. Only continuous data was analysed within this chapter which was tested in accordance to normality (Section 2.5.2). 


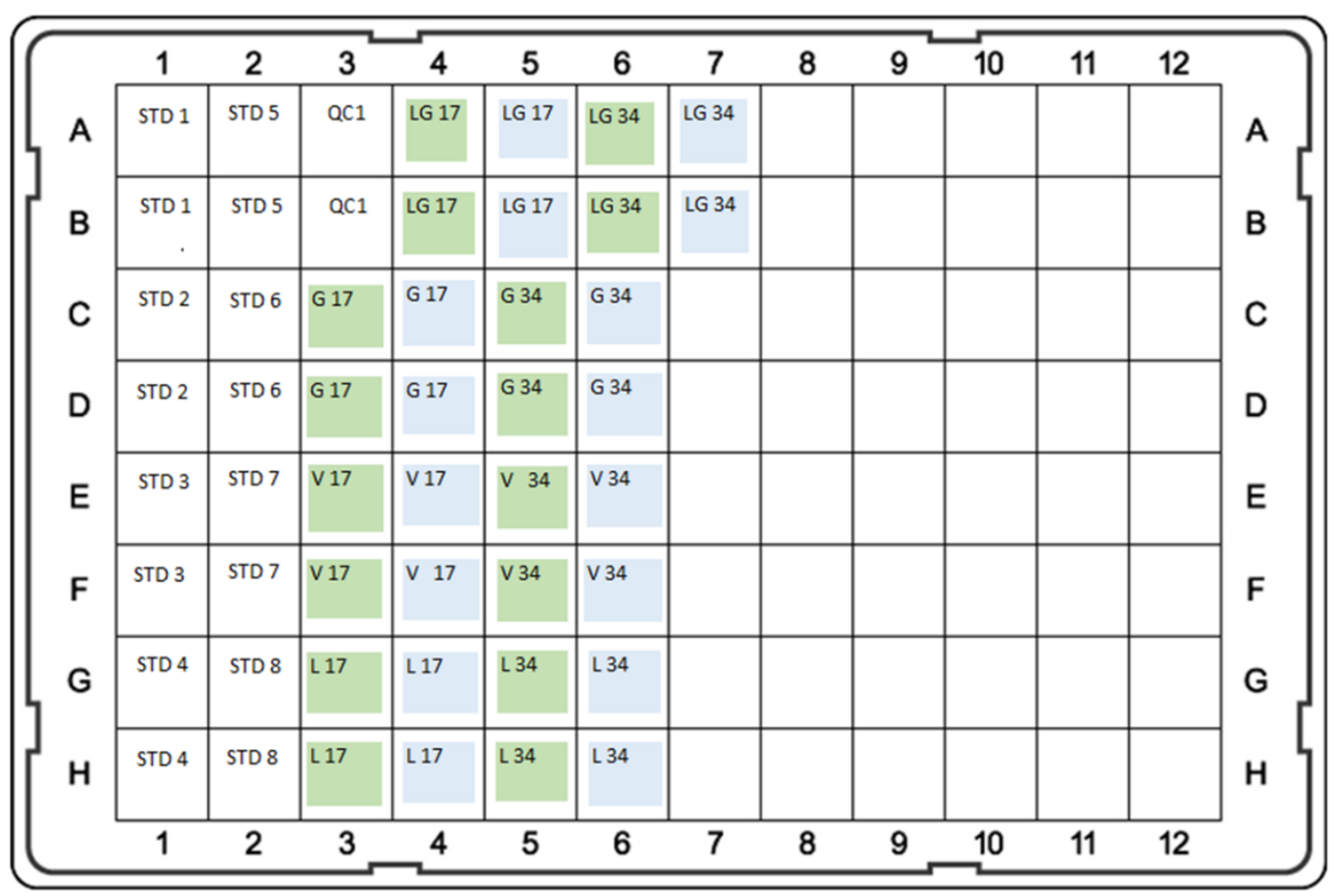

Figure 3.3. Plate layout for adipokine ELISA for each experimental repeat.

STD = standard, $\mathrm{QC}=$ quality control, green boxes indicate hyperglycaemic environment, blue boxes indicate normoglycaemic environment and treatments are denoted by $\mathrm{G}=$ acyl- ghrelin, $\mathrm{V}=$ vehicle, L=LPS and LG=acyl-ghrelin and LPS. 17 and 34 indicate the relevant time point samples where extracted i.e. 17 hours and 34 hours post treatment. 


\subsection{Results}

\subsubsection{Acyl-ghrelin treated human mature adipocytes in a normoglycaemic environment}

\section{Lipid area}

There was a significant increase in lipid area $\left(\mu \mathrm{m}^{2}\right)$ in acyl-ghrelin treated cells when compared to vehicle at both time points (Table 3.1 \& Figure 3.4). Between 17 and 34 hours, acyl-ghrelin mediated lipid retention increased cell lipid area significantly from $4348.0 \mu^{2}$ to $5595.6 \mu^{2}$ respectively $(p<0.01$ ) (Figure $3.4 A \& 3.4 C$ ). However, over the time course, the lipid area of the vehicle treated cells remained unchanged with an area of $3461.4 \mu \mathrm{m}^{2}$ at 17 hours and $3516.5 \mu \mathrm{m}^{2}$ at 34 hours $(p=$ 0.98) (Figure 3.4B).

Cell diameter $(\mu \mathrm{m})$ and lipid area $\left(\mu \mathrm{m}^{2}\right)$ were compared during data analysis to ensure an increase in lipid area was not due to an increase in cellular size. Results confirm no significant correlation between global cell diameter and global lipid area within the normoglycaemic environment of both acyl-ghrelin and vehicle treated cells $(r=-0.003, p=0.95)$ (Table 3.1, Figure 3.5). 


\begin{tabular}{|c|c|c|c|c|c|c|}
\hline \multirow[b]{2}{*}{$\begin{array}{l}\text { Time } \\
\text { point }\end{array}$} & \multicolumn{2}{|c|}{ Lipid area $\left(\mu \mathrm{m}^{2}\right)$} & \multirow[b]{2}{*}{ P-value } & \multicolumn{2}{|c|}{ Cell diameter $(\mu \mathrm{m})$} & \multirow[b]{2}{*}{ P-value } \\
\hline & $\begin{array}{l}\text { Vehicle } \\
\text { treated } \\
(n=600)\end{array}$ & $\begin{array}{l}\text { Acyl-ghrelin } \\
\text { treated } \\
(n=600)\end{array}$ & & $\begin{array}{l}\text { Vehicle } \\
\text { treated } \\
(n=600)\end{array}$ & $\begin{array}{l}\text { Acyl-ghrelin } \\
\text { treated } \\
(n=600)\end{array}$ & \\
\hline $\begin{array}{c}17 \\
\text { hours }\end{array}$ & $\begin{array}{c}3461.4 \\
{[3335-3914]}\end{array}$ & $\begin{array}{c}4348.0 \\
{[4371-4896]}\end{array}$ & $<0.001$ & $\begin{array}{c}176.7 \\
{[152-203]}\end{array}$ & $\begin{array}{c}171.1 \\
{[150-194]}\end{array}$ & 0.44 \\
\hline $\begin{array}{c}34 \\
\text { hours }\end{array}$ & $\begin{array}{c}3516.5 \\
{[3457-3734]}\end{array}$ & $\begin{array}{c}5595.6 \\
{[5254-5742]}\end{array}$ & $<0.001$ & $\begin{array}{c}164.2 \\
{[147-188]}\end{array}$ & $\begin{array}{c}167.3 \\
{[152-194]}\end{array}$ & 0.64 \\
\hline P-value & 0.98 & $<0.01$ & & 0.10 & 0.38 & \\
\hline
\end{tabular}

Table 3.1. Lipid area and cell diameter for acyl-ghrelin treated cells in normoglycaemic environment.

Median and IQR shown as not normally distributed and Kruskal-Wallis used for data analysis. Pvalues $<0.05$ are statistically significant and in bold.
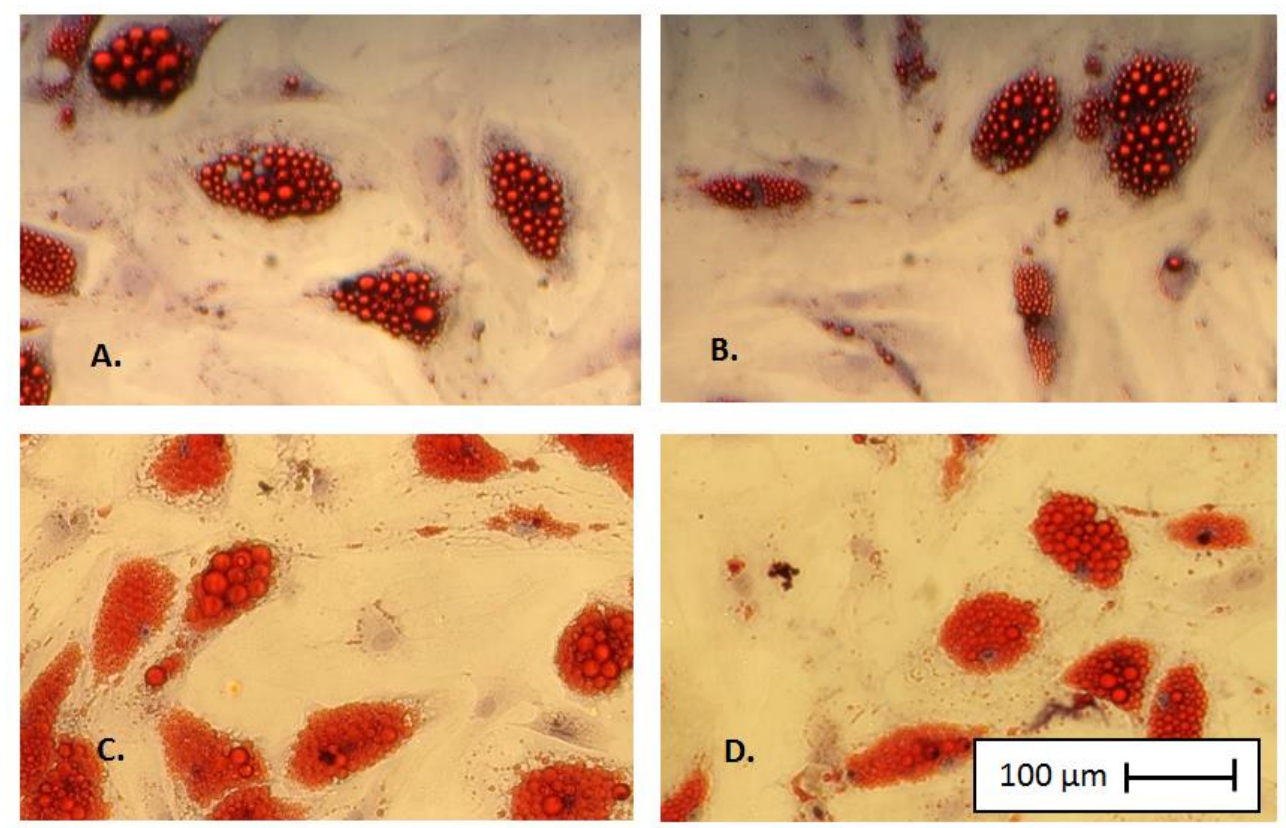

Figure 3.4.A-D. Representative Oil red O imaging of mature SGBS cells in $5.5 \mathrm{mM}$ (normoglycaemia) glucose at x50 magnification. Red stain- lipid droplets; purple stain- nuclei. (A)- Cells grown in presence of acyl-ghrelin for 17 hours. (B)-Cells grown in presence of PBS vehicle for 17 hours. (C)Cells grown in presence of acyl-ghrelin for 34 hours. (D)-Cells grown in presence of PBS vehicle for 34 hours. 


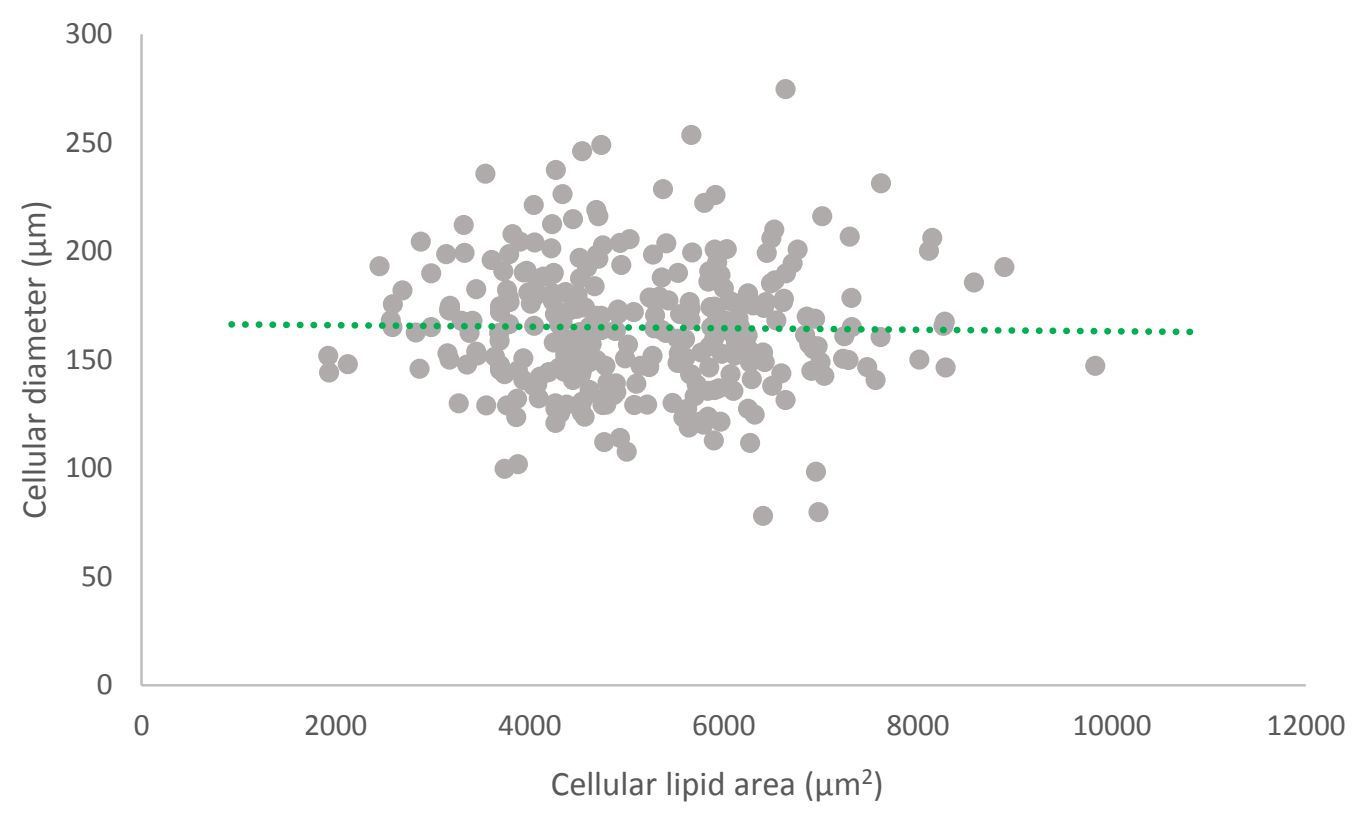

Figure 3.5. Scatter graph between cell diameter $(\mu \mathrm{m})$ and lipid area $\left(\mu \mathrm{m}^{2}\right)$ of all cell treatments in normoglycaemic environment. Pearson's correlation, $r=-0.003, p=0.95 . \quad y=-0.0003 x+166.55$. $\mathrm{R}^{2}=0.0002$

\section{$\underline{\text { Gene expression }}$}

\section{$\underline{A B C G 1}$}

Gene expression data indicated a significant $8.1( \pm 2.1)$-fold down-regulation of $A B C G 1$ in acyl-ghrelin treated cells after 17 hours exposure in comparison to vehicle treated cells $(p<0.001)$. However, at 34 hours, this down-regulation had curtailed, exhibiting no overall change in $A B C G 1$ expression between acyl-ghrelin treated cells and vehicle control $(0.9 \pm 0.4$-fold change; $p=0.15)$ (Figure 3.6A).

\section{$\underline{L X R B}$}

LXRB expression showed no significant change in gene expression at 17 hours in cells exposed to acyl-ghrelin compared to those of vehicle treatment $(0.9 \pm 0.3$-fold 
change; $p=0.45)$. However, at 34 hours post acyl-ghrelin exposure, it exhibited a significant $3.6 \pm 0.3$-fold up regulation $(p<0.001)$ (Figure 3.6B).

\section{$\underline{\text { SREBF1 }}$}

SREBF1 expression in acyl-ghrelin treated cells had a non-significant up-regulation at 17 hours of $1.8 \pm 0.2$-fold change $(p=0.06)$ compared to vehicle treated cells. This increased over the course of the experiment, producing a significant up-regulation in the presence of acyl-ghrelin at 34 hours $(5.9 \pm 1.8$-fold, $p<0.001)$ (Figure $3.6 \mathrm{C})$.

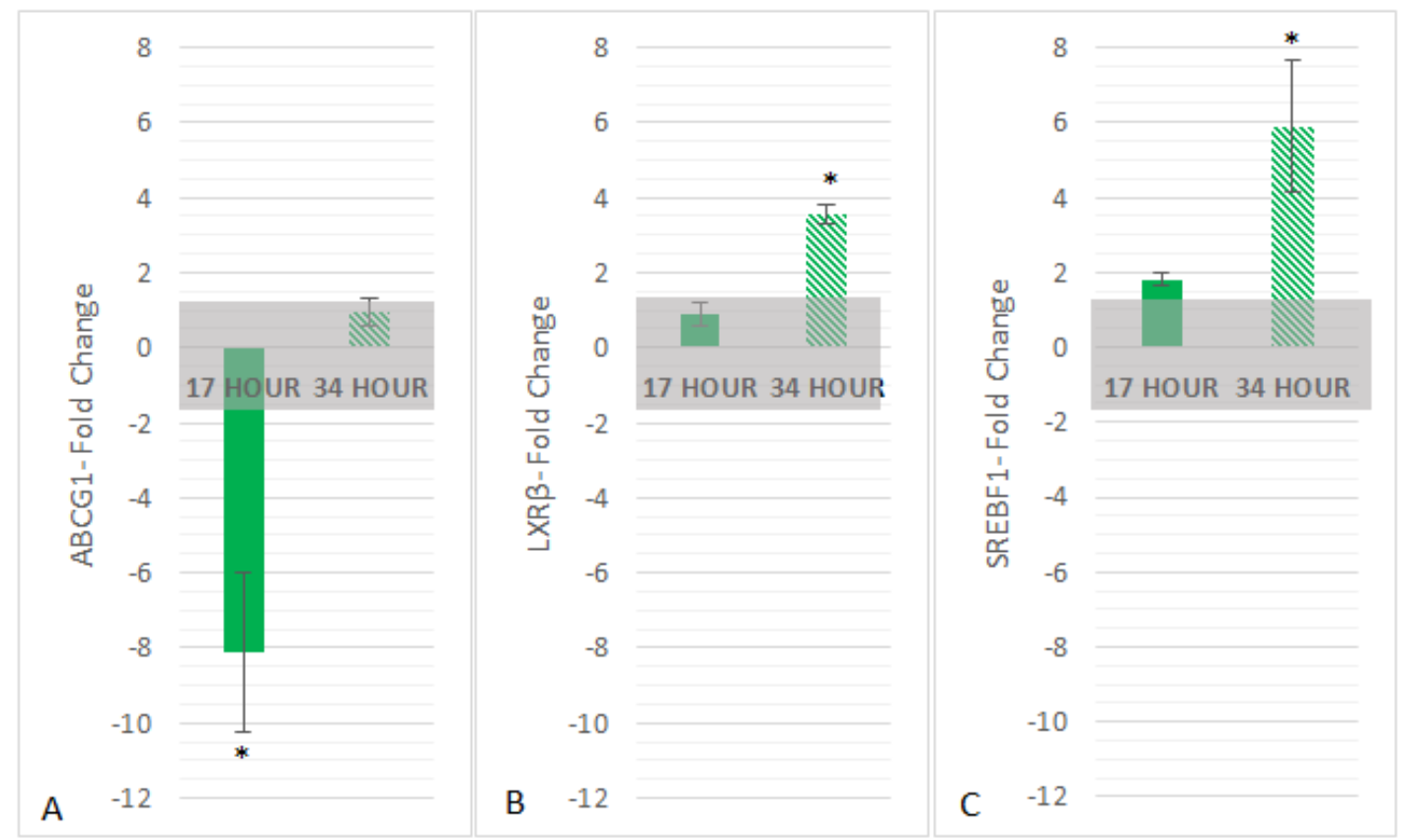

Figure 3.6A-C. Relative fold change values for gene expression data in acyl-ghrelin treated cells in normoglycaemic environment in comparison to vehicle at both time points (17 and 34 hours). Fold change between -1.5 and 1.5 is classed as no relative gene expression change (grey region). (A)ABCG1-fold change, (B)- LXR $\beta$-fold change and (C)-SREBF1-fold change. Error bars indicate standard deviation between $\Delta \Delta C$ t of experimental repeats $(n=6) .{ }^{*}$ indicates a significant $(p<0.05)$ difference of $\Delta \mathrm{Ct}$ in acyl-ghrelin treated cells versus vehicle treated cells, determined using an independent $\mathrm{t}$ test. 


\section{Inflammatory markers}

$\underline{I L-6}$

IL-6 release was measured in cell supernatants after exposure to acyl-ghrelin, LPS or a combination of the two (acyl-ghrelin and LPS) (Table 3.2). Exposure to acyl-ghrelin showed no significant change in IL-6 release in comparison to vehicle treated cell supernatant at 17 hours ( $46.9 \vee 53.0 \mathrm{pg} / \mathrm{mL} ; \mathrm{p}=0.149)$, however at 34 hours there was a significant decline in IL-6 levels in comparison to vehicle treated cell supernatant (74.8 v $94.1 \mathrm{pg} / \mathrm{mL} ; \mathrm{p}<0.05)$. Immune challenging vehicle cells with LPS resulted in a significant increase in IL- 6 at both 17 and 34 hours $(425.1 \mathrm{pg} / \mathrm{mL}$ and $480.3 \mathrm{pg} / \mathrm{mL}$; both $\mathrm{p}<0.05$, respectively). There was no change in IL-6 levels when acyl-ghrelin and LPS exposure was compared to LPS only treatments at both 17 and 34 hours $(412.8 \vee 425.1 \mathrm{pg} / \mathrm{ml} ; \mathrm{p}=0.248$ and $451.6 \vee 480.3 \mathrm{pg} / \mathrm{mL} ; \mathrm{p}=0.08$; respectively).

\begin{tabular}{|c|c|c|}
\cline { 2 - 3 } \multicolumn{1}{c|}{} & \multicolumn{2}{c|}{ IL-6 [pg/mL] } \\
\hline Treatment & $\begin{array}{c}17 \text { hours } \\
(\mathrm{n}=6)\end{array}$ & $\begin{array}{c}34 \text { hours } \\
(\mathrm{n}=6)\end{array}$ \\
\hline Vehicle & $53.0[43.7-54.8]$ & $94.1[89.9-99.7]$ \\
\hline Acyl-ghrelin & $46.9[32.4-48.5]$ & $74.8[67.9-84.3]$ \\
\hline LPS & $425.1[391.2-450.8]$ & $480.3[462.5-519.4]$ \\
\hline LPS \& Acyl-ghrelin & $412.8[401.1-417.2]$ & $451.6[444.1-464.5]$ \\
\hline
\end{tabular}

Table 3.2. IL-6 levels for acyl-ghrelin and vehicle treated cells in normoglycaemic environments. Median and IQR shown as not normally distributed. 
TNF $\alpha$ release were measured in cell supernatants after exposure to acyl-ghrelin, LPS or a combination of the two (acyl-ghrelin and LPS) (Table 3.3). Acyl-ghrelin treatment showed no significant alterations of TNFa levels in cell supernatants at both 17 and 34 hour $(7.3 \mathrm{pg} / \mathrm{mL}$ and $6.2 \mathrm{pg} / \mathrm{mL}: \mathrm{p}=0.245$ and $\mathrm{p}=0.08$, respectively). LPS exposure caused a significant increase in TNF $\alpha$ levels at both 17 and 34 hours (79.0 pg/mL and $78.0 \mathrm{pg} / \mathrm{mL}$ : both $\mathrm{p}<0.05$, respectively). This increase was also present in cell supernatant of cells pre-treated with acyl-ghrelin prior to LPS exposure $(72.3 \mathrm{pg} / \mathrm{mL}$ and $76.2 \mathrm{pg} / \mathrm{mL}$ : both $\mathrm{p}<0.05$, respectively). However, there was no significant change when comparing LPS and combined LPS and acyl-ghrelin treatment on TNF $\alpha$ levels at 17 and 34 hours (LPS $\vee$ LPS\& acyl-ghrelin: $79.0 \mathrm{pg} / \mathrm{mL} v$ $72.3 \mathrm{pg} / \mathrm{mL}$ and $78.0 \mathrm{pg} / \mathrm{mL} \vee 76.2 \mathrm{pg} / \mathrm{mL}: \mathrm{p}=0.08$ and $\mathrm{p}=0.248$, respectively).

\begin{tabular}{|c|c|c|}
\cline { 2 - 3 } \multicolumn{1}{c|}{} & \multicolumn{2}{c|}{ TNF $\alpha[\mathrm{pg} / \mathrm{mL}]$} \\
\hline Treatment & $\begin{array}{c}17 \text { hours } \\
(\mathrm{n}=6)\end{array}$ & 34 hours \\
& $4.0[2.2-7.3]$ & $1.5[1.4-3.1]$ \\
\hline Vehicle & $7.3[6.1-7.7]$ & $6.2[4.6-6.3]$ \\
\hline Acyl-ghrelin & $79.0[74.6-84.7]$ & $78.0[77.7-79.1]$ \\
\hline LPS & $72.3[67.0-77.2]$ & $76.2[70.5-82.2]$ \\
\hline LPS \& Acyl-ghrelin & & \\
\hline
\end{tabular}

Table 3.3. TNF $\alpha$ levels for acyl-ghrelin and vehicle treated cells in normoglycaemic environments. Median and IQR shown as not normally distributed. 
IL-10 levels was measured after exposure to acyl-ghrelin, LPS or a combination of the two (acyl-ghrelin and LPS) (Table 3.4). Mature adipocytes treated with acylghrelin showed a significant increase in the level of IL-10 present in cell supernatants in comparison to the vehicle control at 17 and 34 hours $(314.1 \mathrm{pg} / \mathrm{mL}$ and $341.0 \mathrm{pg} / \mathrm{mL}$ : both $\mathrm{p}<0.001$, respectively). Immune challenged cells demonstrated no overall change in IL-10 levels at both 17 and 34-hour time points (264.3 pg/mL and $196.4 \mathrm{pg} / \mathrm{mL}: \mathrm{p}=0.137$ and $\mathrm{p}=0.934$, respectively). Pre-treatment of cells with acyl-ghrelin before LPS exposure resulted in no change in IL-10 levels compared to cell supernatant of LPS only exposure at 17 hours $(292.3 \mathrm{pg} / \mathrm{mL} \mathrm{v}$ $264.4 \mathrm{pg} / \mathrm{mL} ; \mathrm{p}=0.30$, respectively), and a significant increase at 34 hours (303.9 $\mathrm{pg} / \mathrm{mL}$ v $196.4 \mathrm{pg} / \mathrm{mL} ; \mathrm{p}<0.001$, respectively).

\begin{tabular}{|c|c|c|}
\cline { 2 - 3 } \multicolumn{1}{c|}{} & \multicolumn{2}{c|}{ IL-10 [pg/mL] } \\
\hline Treatment & $\begin{array}{c}17 \text { hours } \\
(\mathrm{n}=6)\end{array}$ & $\begin{array}{c}34 \text { hours } \\
(\mathrm{n}=6)\end{array}$ \\
\hline Vehicle & $226.8(13.7)$ & $188.2(20.8)$ \\
\hline Acyl-ghrelin & $314.1(27.3)$ & $341.0(14.0)$ \\
\hline LPS & $264.3(26.6)$ & $196.4(17.5)$ \\
\hline LPS \& Acyl-ghrelin & $292.3(19.5)$ & $303.9(25.2)$ \\
\hline
\end{tabular}

Table 3.4. IL-10 levels for acyl-ghrelin and vehicle treated cells in normoglycaemic environments. 


\subsubsection{Acyl-ghrelin treated human mature adipocytes in a hyperglycaemic environment}

\section{Lipid area}

Within a hyperglycaemic environment, cells treated with acyl-ghrelin exhibited a significant increase in lipid area $\left(\mu \mathrm{m}^{2}\right)$ when compared to vehicle substitute (Figure 3.7A-D). This marked increase in lipid area was present at both 17 hour $\left(4788.7 \mu \mathrm{m}^{2}\right.$ v $\left.3592.5 \mu \mathrm{m}^{2} ; \mathrm{p}<0.001\right)$ and 34 hour time points $\left(4435.8 \mu \mathrm{m}^{2} \mathrm{v} 3484.0 \mu \mathrm{m}^{2}\right.$; $p<0.001$ ) (Table 3.5). Over the time course, there was no significant increase in cellular lipid area within cells treated with acyl-ghrelin $\left(4788.7 \mu \mathrm{m}^{2} v 4435.8 \mu \mathrm{m}^{2}\right.$, $\mathrm{p}=0.086$ ). Furthermore, cells exposed to the vehicle substitute also showed no

significant intracellular lipid area change, (17 hour v 34 hour; $3592.5 \mu \mathrm{m}^{2}$ v 3484.0 $\left.\mu \mathrm{m}^{2} ; \mathrm{p}=0.174\right)$ (Table 3.5).

When global cell diameter and lipid area were analysed within a hyperglycaemic environment there was no significant correlation between the two, indicating that lipid area was independent of cellular diameter $(r=-0.065, p=0.13)$ (Table 1, Figure 3.8). 


\begin{tabular}{|c|c|c|c|c|c|c|}
\hline \multirow[b]{2}{*}{$\begin{array}{l}\text { Time } \\
\text { point }\end{array}$} & \multicolumn{2}{|c|}{ Lipid area $\left(\mu \mathrm{m}^{2}\right)$} & \multicolumn{3}{|c|}{ Cell diameter $(\mu \mathrm{m})$} & \multirow[b]{2}{*}{ P-value } \\
\hline & $\begin{array}{l}\text { Vehicle } \\
\text { treated } \\
(n=600)\end{array}$ & $\begin{array}{l}\text { Acyl-ghrelin } \\
\text { treated } \\
(n=600)\end{array}$ & P-value & $\begin{array}{l}\text { Vehicle } \\
\text { treated } \\
(n=600)\end{array}$ & $\begin{array}{l}\text { Acyl-ghrelin } \\
\text { treated } \\
(n=600)\end{array}$ & \\
\hline $\begin{array}{c}17 \\
\text { hours }\end{array}$ & $\begin{array}{c}3592.5 \\
{[2491-4696]}\end{array}$ & $\begin{array}{c}4788.7 \\
{[4040-5976]}\end{array}$ & $<0.001$ & $\begin{array}{c}175.9 \\
{[154-204]}\end{array}$ & $\begin{array}{c}170.0 \\
{[131-210]}\end{array}$ & 0.28 \\
\hline $\begin{array}{c}34 \\
\text { hours }\end{array}$ & $\begin{array}{c}3484.0 \\
{[2892-4005]}\end{array}$ & $\begin{array}{c}4435.8 \\
{[3828-5416]}\end{array}$ & $<0.001$ & $\begin{array}{c}178.9 \\
{[156-208]}\end{array}$ & $\begin{array}{c}166.4 \\
{[138-191]}\end{array}$ & 0.10 \\
\hline P-value & 0.17 & 0.09 & & 0.54 & 0.55 & \\
\hline
\end{tabular}

Table 3.5. Lipid area and cell diameter for acyl-ghrelin and vehicle treated cells in hyperglycaemic environment. Median and IQR shown as not normally distributed and Kruskal-Wallis used for data analysis. P-values $<0.05$ are statistically significant are in bold.
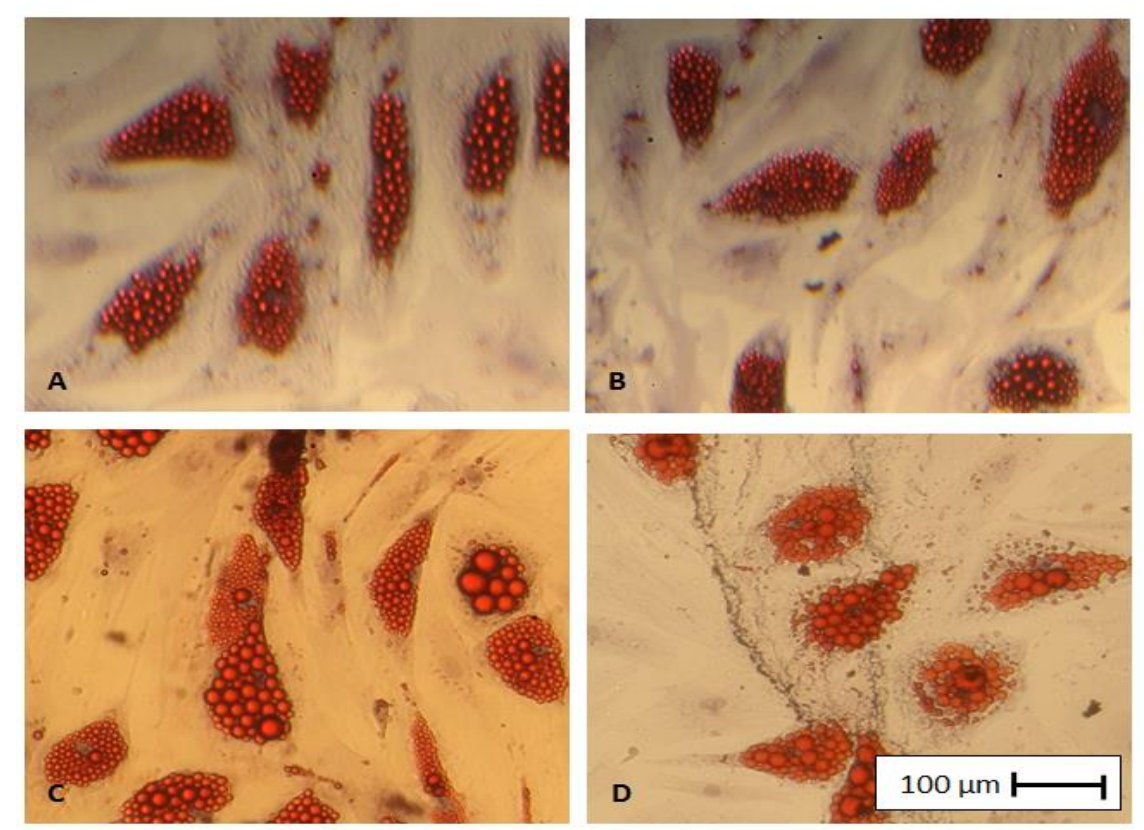

Figure 3.7 A-D. Representative Oil red O imaging of mature SGBS cells in $25 \mathrm{mM}$ (hyperglycaemia) glucose at x50 magnification. Red stain- lipid droplets; purple stain- nuclei. (A)- Cells grown in presence of acyl-ghrelin for 17 hours. (B)- Cells grown in presence of PBS vehicle for 17 hours. (C)Cells grown in presence of acyl-ghrelin for 34 hours. (D)- Cells grown in presence of PBS vehicle for 34 hours. 


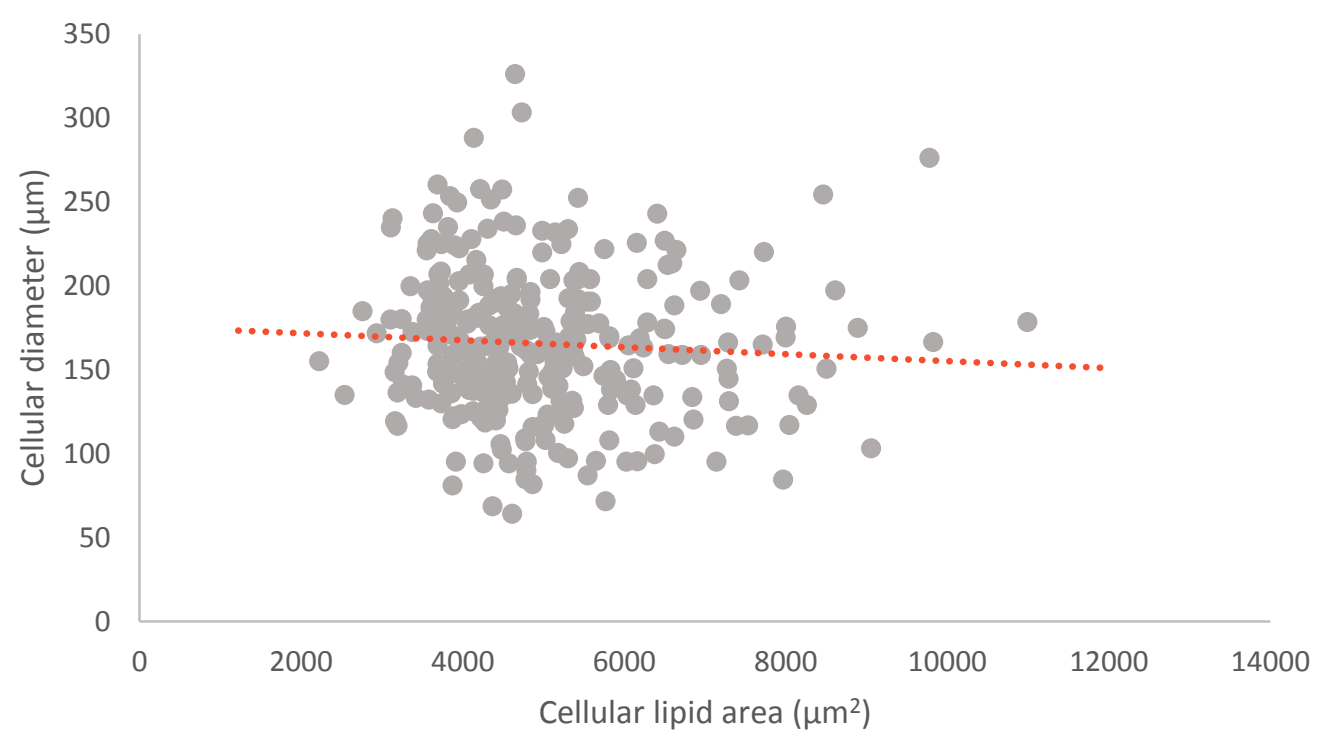

Figure 3.8. Scatter graph between cell diameter $(\mu \mathrm{m})$ and lipid area $\left(\mu \mathrm{m}^{2}\right)$ of all cell treatments in a hyperglycaemic environment. Pearson's correlation, $r=-0.065, p=0.13 . y=-0.0021 x+175.83 . R^{2}=$ 0.0043 .

\section{Gene expression}

\section{$\underline{A B C G 1}$}

$A B C G 1$ showed an initial non-significant down regulation of $3.0( \pm 1.3)$-fold change at 17 hour $(p=0.05)$, however acyl-ghrelin exposure over time produced a significant down regulatory effect on $A B C G 1$ producing a $6.2( \pm 2.2)$-fold change at 34 hours $(p<0.05)$ (Figure 3.9A).

$\underline{L X R B}$

LXRB showed a significant initial down regulation of $2.9( \pm 0.7)$-fold change $(p<0.01)$ at 17 hours within cells exposed to acyl-ghrelin, this effect was enhanced over the time course of the experiment, demonstrating acyl-ghrelin exposure for 34 hours 
caused a further decrease in the down regulation of $L X R B(10.8( \pm 3.4)$-fold change; $\mathrm{p}<0.001$ ) (Figure 3.9B).

\section{SREBF1}

SREBF1 expression was not significantly altered within cells treated with acylghrelin in comparison to those treated with a vehicle exhibiting a fold change of 1.4 $( \pm 0.1)$ at 17 hours $(p=0.24)$ and $1.2( \pm 0.1)$ at 34 hours $(p=0.43)$ (Figure $3.9 C)$.

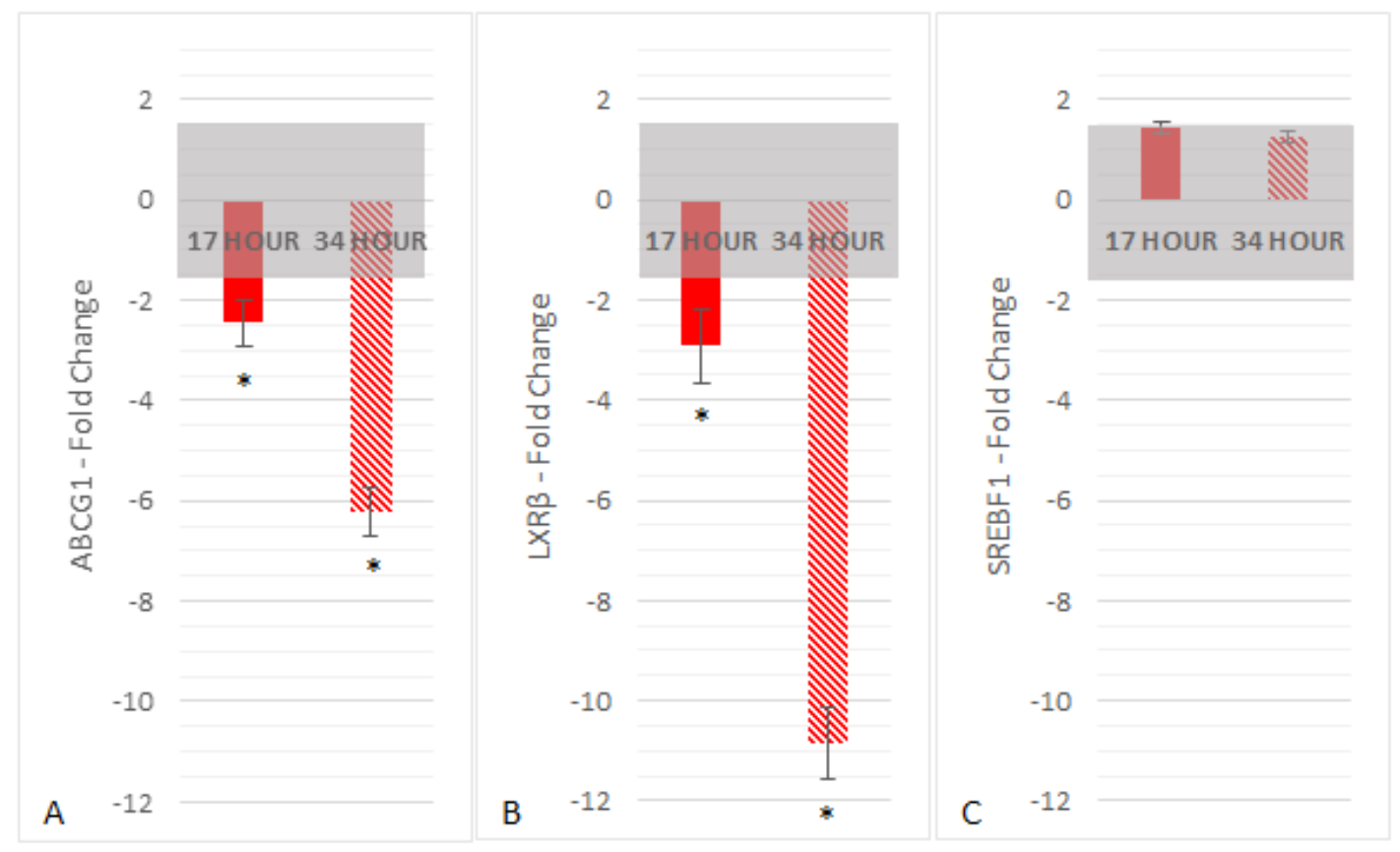

Figure 3.9.A-C. Relative fold change values for gene expression data in acyl-ghrelin treated cells in hyperglycaemic environment in comparison to vehicle at both time points (17 and 34 hours). Fold change between -1.5 and 1.5 is classed as no relative gene expression change (grey region). (A)ABCG1-fold change, (B)- LXRß-fold change and (C)-SREBF1-fold change. Error bars indicate standard deviation between $\Delta \Delta \mathrm{Ct}$ of experimental repeats $(n=6) .{ }^{*}$ indicates a significant $(p<0.05)$ difference of $\Delta \mathrm{Ct}$ in acyl-ghrelin treated cells versus vehicle treated cells, determined using an independent $\mathrm{t}$ test. 


\section{Inflammatory markers}

$\underline{I L-6}$

IL-6 levels within a hyperglycaemic environment were compared across all treatments (Table 3.6). Cells treated with acyl-ghrelin only showed no significant change in IL-6 levels at 17 hours (75.5 v $89.5 \mathrm{pg} / \mathrm{mL} ; \mathrm{p}=0.149)$, however at 34 hours there was a significant decline in IL-6 levels in comparison to vehicle treated cell supernatant $(107.2 \vee 147.0 \mathrm{pg} / \mathrm{mL} ; \mathrm{p}<0.05)$. LPS exposure resulted in a significant increase in IL-6 at both 17 and 34 hours $(561.5 \mathrm{pg} / \mathrm{mL}$ and $650.0 \mathrm{pg} / \mathrm{mL}$; both $p<0.05$, respectively). Pre-treatment of the cells to acyl-ghrelin before administering LPS also indicated a significant increase in IL-6 at both 17 and 34 hours $(528.0 \mathrm{pg} / \mathrm{mL}$ and $521.4 \mathrm{pg} / \mathrm{mL}$; both $\mathrm{p}<0.05)$. Cells treated with acyl-ghrelin prior to LPS exposure showed a significant decline in IL-6 levels in comparison to cell supernatant of LPS only treated cells across both timepoints (17 hour: 528.0 $\mathrm{pg} / \mathrm{mL} \vee 561.5 \mathrm{pg} / \mathrm{mL}$ and 34 hours: $521.4 \mathrm{pg} / \mathrm{mL} \vee 650.0 \mathrm{pg} / \mathrm{mL}$; both $\mathrm{p}<0.05$; respectively).

\begin{tabular}{|c|c|c|}
\cline { 2 - 3 } \multicolumn{1}{c|}{} & \multicolumn{2}{c|}{$\mathrm{IL}-6[\mathrm{pg} / \mathrm{mL}]$} \\
\hline Treatment & $\begin{array}{c}17 \text { hours } \\
(\mathrm{n}=6)\end{array}$ & $\begin{array}{c}34 \text { hours } \\
(\mathrm{n}=6)\end{array}$ \\
\hline Vehicle & $89.5[87.7-95.5]$ & $147.0[138.7-151.7]$ \\
\hline Acyl-ghrelin & $75.5[69.0-89.5]$ & $107.2[94.0-121.4]$ \\
\hline LPS & $561.5[547.4-574.1]$ & $650.0[638.3-653.9]$ \\
\hline LPS \& Acyl-ghrelin & $528.0[515.8-540.3]$ & $521.4[511.1-533.8]$ \\
\hline
\end{tabular}

Table 3.6. IL-6 levels for acyl-ghrelin and vehicle treated cells in hyperglycaemic environment. Median and IQR shown as not normally distributed. 
TNF $\alpha$ levels within cell supernatants was measured after exposure to acyl-ghrelin, LPS or both LPS and acyl-ghrelin (Table 3.7). Exposure to acyl-ghrelin deemed no significant alterations in TNF $\alpha$ levels at both time points (17 hours: $9.5 \mathrm{pg} / \mathrm{mL}$ and 34 hours: $8.9 \mathrm{pg} / \mathrm{mL}: \mathrm{p}=0.248$ and $\mathrm{p}=0.245$, respectively). Cell exposure to LPS produced a significant increase in cell supernatant TNF $\alpha$ levels at 17 and 34 hours $(80.7 \mathrm{pg} / \mathrm{mL}$ and 78.9 , respectively: both $\mathrm{p}<0.05$, ). Furthermore, in comparison to vehicle treated cells, a significant increase was present when cells were pre-treated with acyl-ghrelin before LPS exposure (17 hours: $77.0 \mathrm{pg} / \mathrm{mL}$ and 34 hours: 75.3 $\mathrm{pg} / \mathrm{mL}$ : both $\mathrm{p}<0.05$, respectively). Pre-treatment with acyl-ghrelin within LPS exposure did not alter the cellular release of TNFa across 17 and 34-hour time points (LPS v LPS\&Ghrelin: $77.0 \mathrm{pg} / \mathrm{mL} \vee 80.7 \mathrm{pg} / \mathrm{mL}$ and $78.9 \mathrm{pg} / \mathrm{mL} \vee 75.3 \mathrm{pg} / \mathrm{mL}$ : $p=0.343$ and $p=0.248$, respectively). The data indicate that acyl-ghrelin had no significant effect on TNFa levels within cell supernatants across either time point within a hyperglycaemic environment, with or without immune challenge.

\begin{tabular}{|c|c|c|}
\cline { 2 - 3 } \multicolumn{1}{c|}{} & \multicolumn{2}{c|}{ TNF $\alpha$ [pg/mL] } \\
\hline Treatment & $\begin{array}{c}17 \text { hours } \\
(\mathrm{n}=6)\end{array}$ & $\begin{array}{c}34 \text { hours } \\
(\mathrm{n}=6)\end{array}$ \\
\hline Vehicle & $5.7[1.8-9.3]$ & $6.4[5.3-7.0]$ \\
\hline Acyl-ghrelin & $9.5[8.4-10.8]$ & $8.9[6.4-10.8]$ \\
\hline LPS & $80.7[79.5-83.4]$ & $78.9[72.7-86.2]$ \\
\hline LPS \& Acyl-ghrelin & $77.0[72.5-81.4]$ & $75.3[74.2-76.8]$ \\
\hline
\end{tabular}

Table 3.7. TNF $\alpha$ levels for acyl-ghrelin and vehicle treated cells in hyperglycaemic environment. Median and IQR shown as not normally distributed. 
Data for IL-10 levels were analysed post acyl-ghrelin, LPS or both LPS and acylghrelin exposure (Table 3.8). Cells exposed to acyl-ghrelin demonstrated no change in cell supernatant IL-10 levels at both 17 hours $(275.2 \mathrm{pg} / \mathrm{mL} ; \mathrm{p}=0.946)$ and 34 hours (378.2 pg/mL; $p=0.560)$. At 17 hours, immune challenge with LPS exposure demonstrated no overall change in IL-10 levels within cell supernatants (234.6 $\mathrm{pg} / \mathrm{mL} ; \mathrm{p}=0.682$ ), however at 34 hours there was a marked decrease in IL-10 levels in comparison to vehicle treated cells $(178.4 \mathrm{pg} / \mathrm{mL} ; \mathrm{p}<0.001)$. Mature adipocytes pre-treated with acyl-ghrelin prior to immune challenge resulted in a significant decline in IL-10 levels at 17 hours in comparison to LPS only, and no significant difference at 34 hours $(140.0 \pm 23.4 \mathrm{pg} / \mathrm{mL} \vee 234.6 \pm 34.3 \mathrm{pg} / \mathrm{mL}$ and $173.1 \pm 14.9$ $\mathrm{pg} / \mathrm{mL} \vee 178.4 \pm 17.5 \mathrm{pg} / \mathrm{mL} ; \mathrm{p}<0.05$ and $\mathrm{p}=0.91$, respectively).

\begin{tabular}{|c|c|c|}
\cline { 2 - 3 } \multicolumn{1}{c|}{} & \multicolumn{2}{c|}{$\mathrm{IL}-10 \mathrm{pg} / \mathrm{mL}]$} \\
\hline Treatment & $\begin{array}{c}17 \text { Hours } \\
(\mathrm{n}=6)\end{array}$ & 34 Hours \\
& $261.9(20.3)$ & $361.6(14.9)$ \\
\hline Vehicle & $275.2(50.9)$ & $378.2(22.1)$ \\
\hline Acyl-ghrelin & $234.6(34.3)$ & $178.4(17.5)$ \\
\hline LPS & $140.0(23.4)$ & $173.1(14.9)$ \\
\hline LPS \& Acyl-ghrelin & & \\
\hline
\end{tabular}

Table 3.8. IL-10 levels for acyl-ghrelin and vehicle treated cells in hyperglycaemic environment. 


\subsubsection{Comparison of glycaemic environment on acyl-ghrelin treated human mature adipocytes}

This section compares lipid retention and inflammatory markers present within cells grown in either a high glucose $[25 \mathrm{mM}]$ or normal glucose [5.5mM] environments to mimic that of circulating blood glucose levels within a hyper- and normoglycaemic state, respectively.

\section{Lipid area}

Data analysis for determining the effect of differences relating to glycaemic environment on cellular lipid area were undertaken. At 17 hours, within the vehicle treated mature adipocytes, the cellular lipid area was independent of glycaemic environment, showing no significant change between hyper- and normoglycaemic treatments (3592.5 [2491 - 4696]. vs. 3461.4 [2502 - 4511], respectively; $p=0.62)$. However, acyl-ghrelin treated cells within hyperglycaemic condition showed a significantly increased lipid area when compared to those grown in a normoglycaemic condition (4788.7 [4040 - 5975]. vs. 4348.0 [3693 - 5727], respectively; $p<0.05$ ) (Figure 3.10 ).

At 34 hours, vehicle treated cells demonstrated a no change in lipid area in the hyperglycaemic environment compared to normoglycaemia (3483.9 [2892 4005]. vs. 3516.5 [3156 - 4114], respectively; $p=0.28$ ). In acyl-ghrelin treated mature adipocytes, there was a significant decrease in the accumulated lipid area in the hyperglycaemic environment in comparison to the normoglycaemic environment (4435.8 [3828 - 5416]. vs. 5595.6 [5254 - 5742], respectively; $p<0.001$ ) (Figure 3.11). 


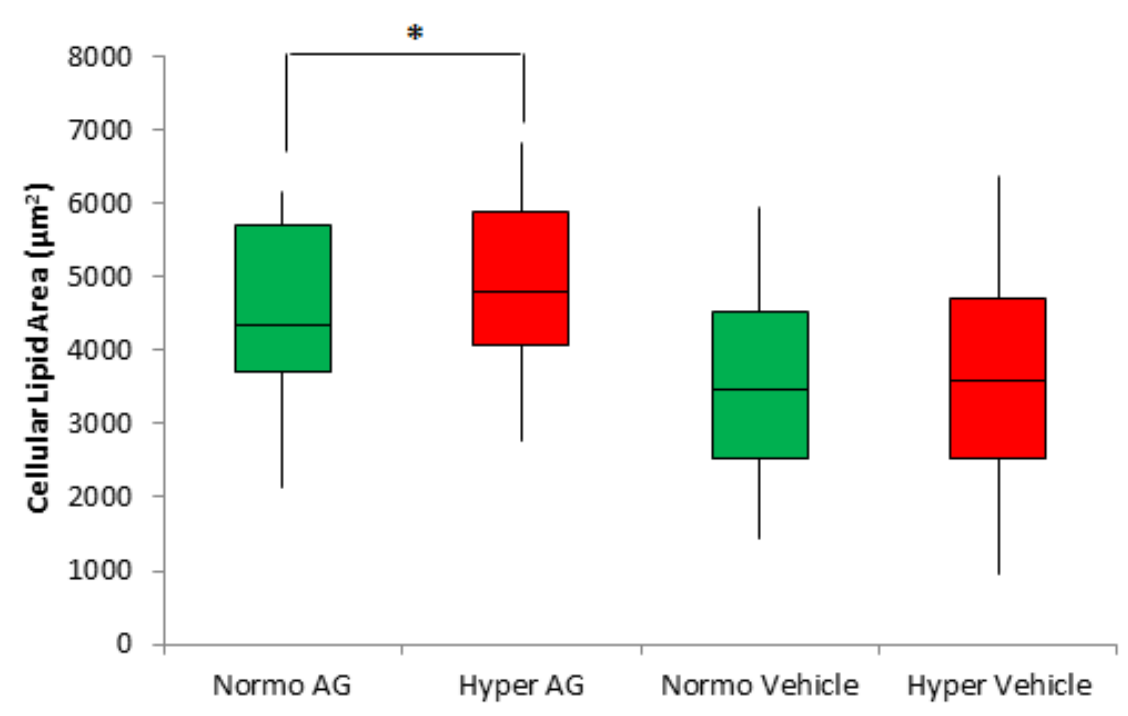

Figure 3.10. Cellular lipid area for acyl-ghrelin and vehicle treated cells within both $5.5 \mathrm{mM}$ (normoglycaemia) and $25 \mathrm{mM}$ (hyperglycaemia) glucose for 17 hours. * indicate statistical significance $(p<0.05)$ determined via Kruskal-Wallis. . $n=600$ per variable. AG- acyl-ghrelin.

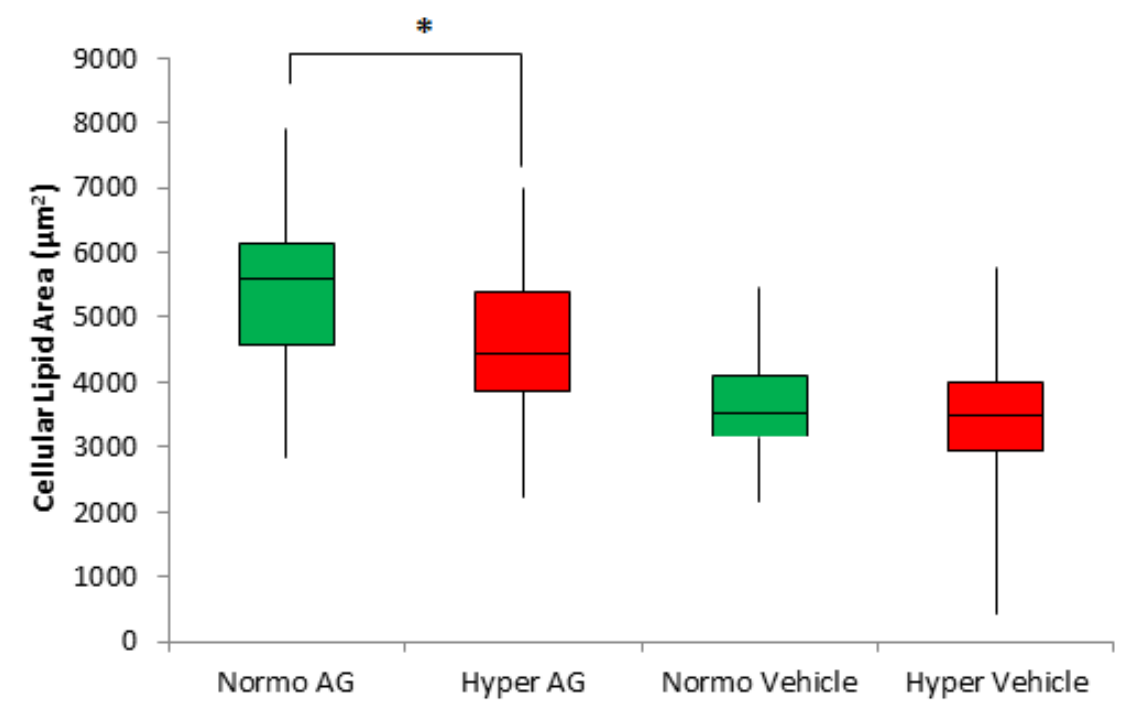

Figure 3.11. Cellular lipid area for acyl-ghrelin and vehicle treated cells within both $5.5 \mathrm{mM}$ (normoglycaemia) and $25 \mathrm{mM}$ (hyperglycaemia) glucose for 34 hours. * indicate statistical significance $(p<0.05)$ determined via Kruskal-Wallis. $n=600$ per variable. AG- acyl-ghrelin. 


\section{Gene expression}

Gene expression analysis used the $2^{-\Delta \Delta C t}$ method, where normalised Ct values from each treatment (acyl-ghrelin or vehicle exposure) group were individually compared, in relation to normoglyceamic against hyperglycaemic environments.

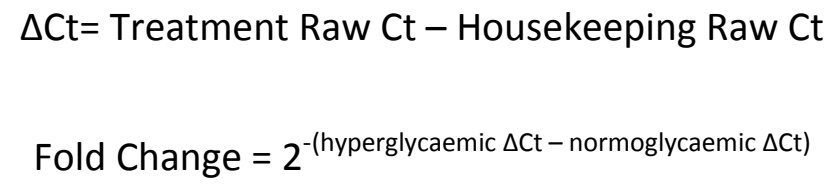

\section{$\underline{A B C G 1}$}

Comparison of the hyperglycaemic to normoglycaemic environment in vehicle treated cells, indicates an initial significant down regulation of $A B C G 1$ after 17 hours $(14.0( \pm 0.3)$-fold; $p<0.001)$. At 34 hours, vehicle treated cells demonstrated a significant down regulation of $5.6( \pm 0.9)$-fold change $(p<0.01)$ however, it is clear to see a curtailment in the magnitude of the effect of a hyperglycaemic environment on $A B C G 1$ expression over time (Figure 3.12A). Exposure to acyl-ghrelin showed a converse effect of hyperglycaemic environment on lipid retention genes to that seen within a vehicle control. $A B C G 1$ expression exhibits a significant down regulation of $4.3( \pm 0.2)$-fold change $(p<0.001)$ at 17 hours and a significant 29.3 -fold change $( \pm 0.8) \quad(p<0.001)$ down regulation at 34 hours' post acyl-ghrelin administration in a hyperglycaemic environment in comparison to a normoglycaemic environment (Figure 3.13A). 
LXRB expression mirrored the gene expression pattern exhibited by $A B C G 1$ when the effect of a hyperglycaemic environment was compared to normoglycaemia. Vehicle treated cells showed a marked decrease in the expression of $L X R B$ at both 17 and 34 hours $(14.6( \pm 0.4) \& 8.3( \pm 1.0)$-fold change; $p<0.001$ and $p<0.001$, respectively) (Figure 3.12B). However, within cells exposed to acyl-ghrelin in a hyperglycaemic environment there was a significant down regulation of $L X R B$ in comparison to those exposed to acyl-ghrelin within a normoglycaemic environment across both time points, with the magnitude of the effect increasing over time (17 hours \& 34 hours; $4.8( \pm 0.6) \& 34.9( \pm 0.7)$-fold change, respectively; both $(p<0.001))$ (Figure 3.13B).

\section{SREBF1}

Vehicle treated cells demonstrated that a hyperglycaemic environment was producing a significant reduction in SREBF1 expression at 17 hours $(13.3( \pm 0.7)$-fold change; $p<0.001)$. Furthermore, this reduction in expression was also present at a diminished but significant level at 34 hours (4.2 $( \pm 0.3)$-fold change; $p<0.001)$ (Figure 3.12C). SREBF1 also showed a further down regulation from 17 hours to 34 hours' post treatment when acyl-ghrelin exposure was combined with a hyperglycaemic environment (17 hours \& 34 hours; $5.2( \pm 0.7)$ \& $30.1( \pm 0.7)$-fold change, respectively; both $p<0.001$ ) (Figure $3.13 \mathrm{C}$ ). 

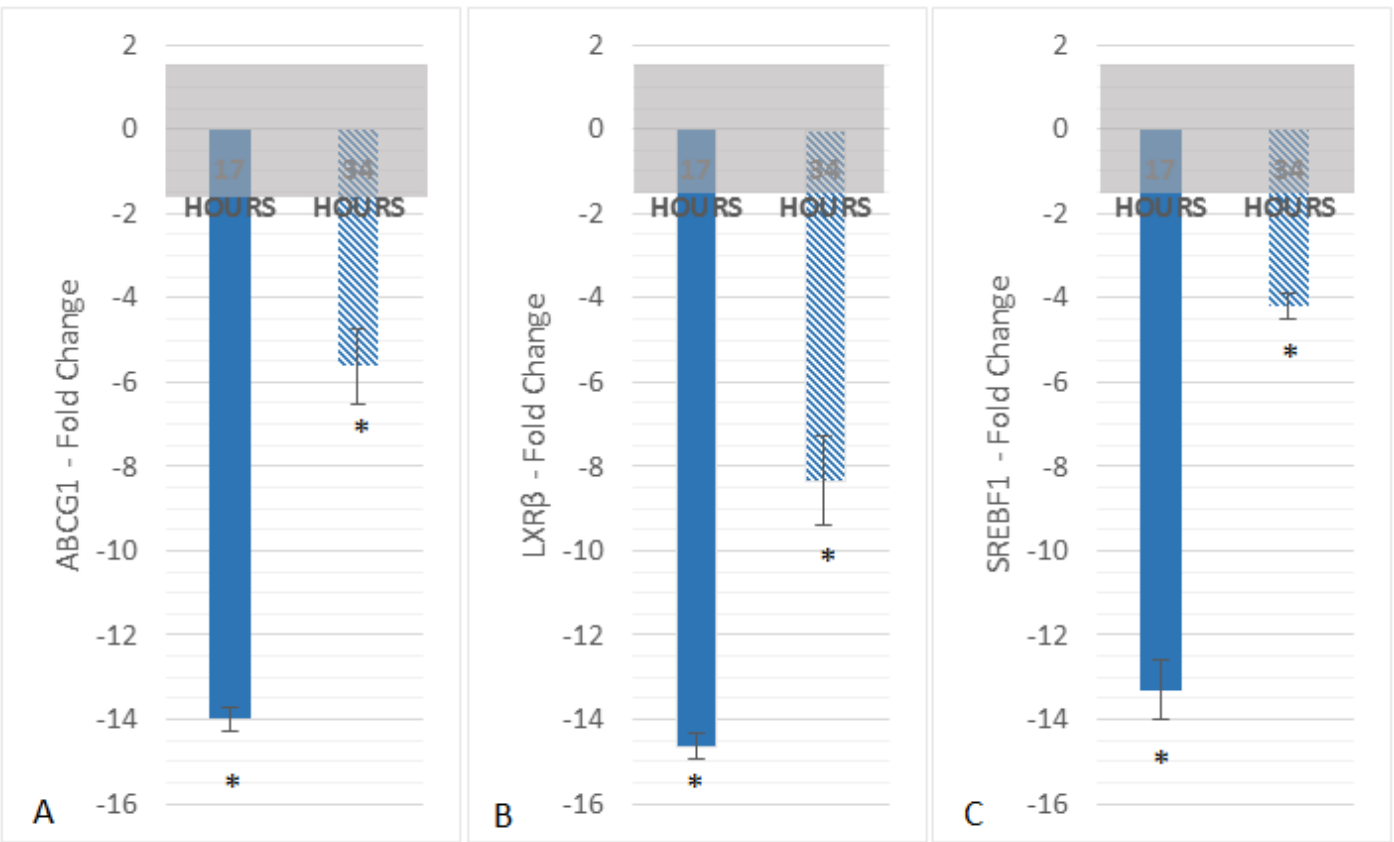

Figure 3.12A-C. Relative fold change values for gene expression data in vehicle treated cells in hyperglycaemic environment in comparison to normoglycaemic environment at both time points (17 and 34 hours). Fold change between -1.5 and 1.5 is classed as no relative gene expression change (grey region). (A)- ABCG1-fold change, (B)- LXRß-fold change and (C)- SREBF1-fold change. Error bars indicate standard deviation between $\Delta \Delta \mathrm{Ct}$ of experimental repeats $(\mathrm{n}=6) .{ }^{*}$ indicates a significant $(\mathrm{p}<0.05)$ difference of $\Delta \mathrm{Ct}$ in acyl-ghrelin treated cells versus vehicle treated cells, determined using an independent t-test. 


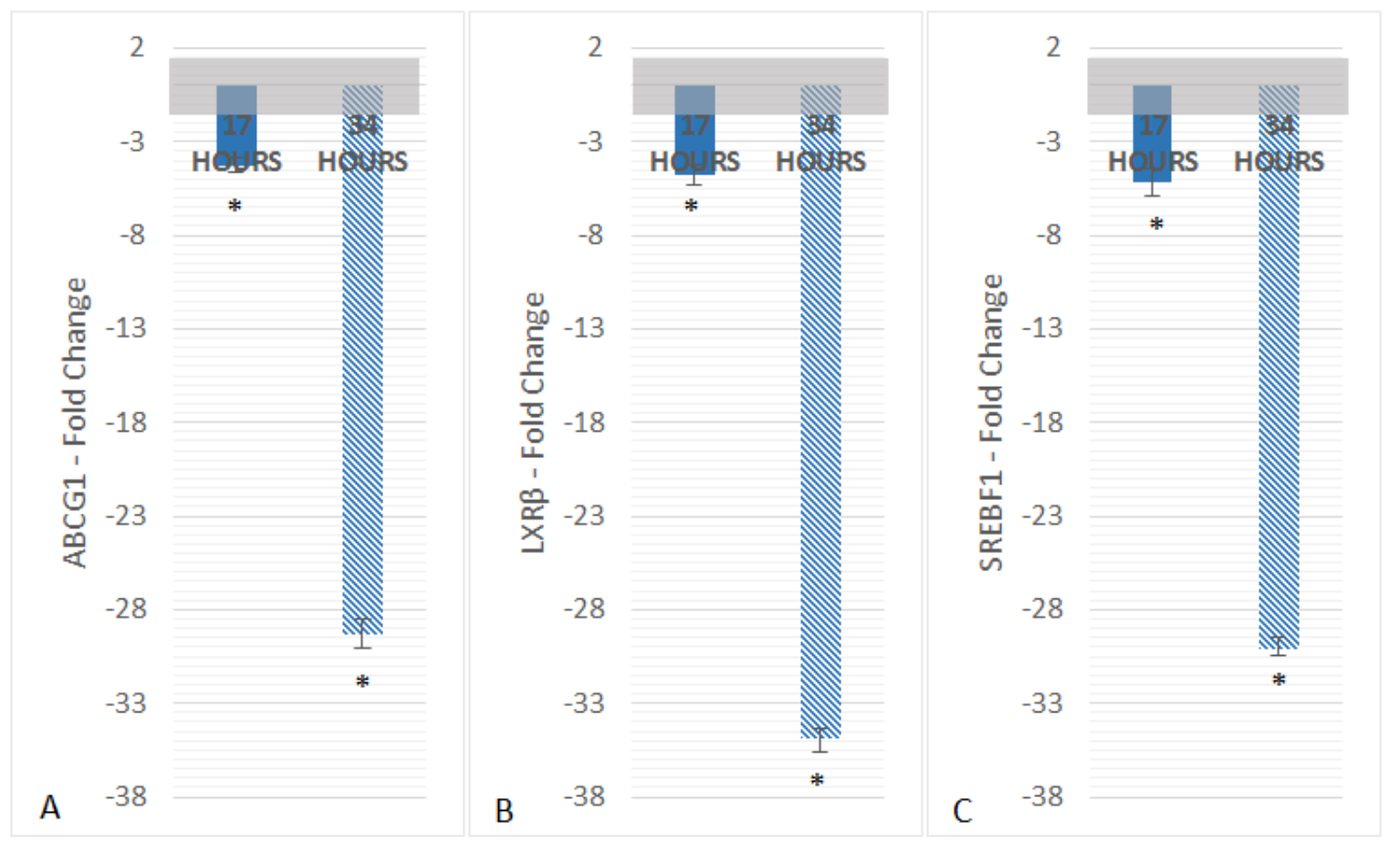

Figure 3.13A-C Relative fold change values for gene expression data in acyl-ghrelin treated in chronic hyperglycaemic environment in comparison to normoglycaemic environment at both time points (17 and 34 hours). Fold change between -1.5 and 1.5 is classed as no relative gene expression change (grey region). (A)- ABCG1-fold change, (B)- LXRß-fold change and (C)- SREBF1-fold change. Error bars indicate standard deviation between $\Delta \Delta C$ t of experimental repeats $(n=6) .{ }^{*}$ indicates a significant $(p<0.05)$ difference of $\Delta C t$ in acyl-ghrelin treated cells versus vehicle treated cells, determined using an independent t-test. 


\section{Inflammatory markers}

$\underline{I L-6}$

IL-6 levels for each treatment at both time points was analysed in comparison to glycaemic environment to establish whether there was a glycaemic effect. A hyperglycaemic environment produced a significant increase in the cell supernatant level of IL-6 across all treatments at 17 hours (Table 3.9) and 34 hours (Table 3.10).

\begin{tabular}{|c|c|c|c|}
\cline { 2 - 4 } \multicolumn{1}{c|}{} & \multicolumn{2}{c|}{$\mathrm{IL}-6[\mathrm{pg} / \mathrm{mL}]$} & \\
\hline Treatment & $\begin{array}{c}\text { Normoglycaemic } \\
(\mathrm{n}=6)\end{array}$ & $\begin{array}{c}\text { Hyperglycaemic } \\
(\mathrm{n}=6)\end{array}$ & P-Value \\
\hline Vehicle & $52.9[43.7-54.8]$ & $89.5[87.7-95.5]$ & $\mathbf{0 . 0 2}$ \\
\hline Acyl-ghrelin & $46.9[32.4-48.5]$ & $75.5[69.0-89.5]$ & $\mathbf{0 . 0 2}$ \\
\hline LPS & $427.1[391.2-450.8]$ & $561.5[547.4-574.1]$ & $\mathbf{0 . 0 2}$ \\
\hline LPS \& acyl-ghrelin & $412.8[401.1-417.2]$ & $528.0[515.8-540.4]$ & $\mathbf{0 . 0 2}$ \\
\hline
\end{tabular}

Table 3.9. Comparison of IL-6 levels in different environments at 17 hours. Statistical analysis conducted using Kruskal-Wallis test. P-value less than 0.05 deemed significant and are in bold.

\begin{tabular}{|c|c|c|c|}
\hline \multirow[b]{2}{*}{ Treatment } & \multicolumn{2}{|c|}{$\mathrm{IL}-6[\mathrm{pg} / \mathrm{mL}]$} & \multirow[b]{2}{*}{ P-Value } \\
\hline & $\begin{array}{c}\text { Normoglycaemic } \\
\qquad(n=6)\end{array}$ & $\begin{array}{l}\text { Hyperglycaemic } \\
\qquad(n=6)\end{array}$ & \\
\hline Vehicle & 94.1 [89.9-99.7] & $147.0[138.7-151.7]$ & 0.02 \\
\hline Acyl-ghrelin & $74.8[68.0-84.3]$ & $107.2[94.0-121.4]$ & 0.02 \\
\hline LPS & 480.3 [462.5-519.4] & $650.0[638.3-653.9]$ & 0.02 \\
\hline LPS \& acyl-ghrelin & 451.6 [444.1-464.5] & $521.4[511.1-533.8]$ & 0.02 \\
\hline
\end{tabular}

Table 3.10. Comparison of IL-6 levels in different environments at 34 hours. Statistical analysis conducted using Kruskal-Wallis test. P-value less than 0.05 deemed significant and are in bold. 
TNF $\alpha$ levels were analysed in comparison of glycaemic environments across all treatments and time points. Mostly, TNF $\alpha$ levels were not significantly altered within cell supernatants for treatments when comparing hyper- to normoglycaemic environment at 17 hours (Table 3.11 ) or 34 hours (Table 3.12). However, within acyl-ghrelin treated mature adipocytes there was a significant increase in TNF $\alpha$ levels at 17 hours when grown in a hyperglycaemic environment $(p<0.05)$.

\begin{tabular}{|c|c|c|c|}
\cline { 2 - 4 } \multicolumn{1}{c|}{} & \multicolumn{2}{c|}{ TNF $\alpha[\mathrm{pg} / \mathrm{mL}]$} & \multicolumn{1}{c|}{} \\
\hline \multirow{2}{*}{ Treatment } & $\begin{array}{c}\text { Normoglycaemic } \\
(\mathrm{n}=6)\end{array}$ & $\begin{array}{c}\text { Hyperglycaemic } \\
(\mathrm{n}=6)\end{array}$ & P-Value \\
\hline Vehicle & $4.0[2.2-7.3]$ & $2.0[1.8-2.7]$ & 0.25 \\
\hline Acyl-ghrelin & $7.3[6.1-7.7]$ & $9.5[8.4-10.8]$ & 0.02 \\
\hline LPS & $78.9[74.6-84.7]$ & $80.7[79.5-83.4]$ & 0.77 \\
\hline LPS \& acyl-ghrelin & $72.3[67.0-77.2]$ & $77.0[72.5-81.4]$ & 0.25 \\
\hline
\end{tabular}

Table 3.11. Comparison of TNF $\alpha$ levels in different environments at 17 hours. Statistical analysis conducted using Kruskal-Wallis test. P-value less than 0.05 deemed significant and are in bold. 


\begin{tabular}{|c|c|c|c|}
\hline \multirow[b]{2}{*}{ Treatment } & \multicolumn{2}{|c|}{ TNF $\alpha[\mathrm{pg} / \mathrm{mL}]$} & \multirow[b]{2}{*}{ P-Value } \\
\hline & $\begin{array}{c}\text { Normoglycaemic } \\
\qquad(n=6)\end{array}$ & $\begin{array}{l}\text { Hyperglycaemic } \\
\qquad(n=6)\end{array}$ & \\
\hline Vehicle & $1.5[1.5-2.1]$ & $2.4[2.3-3.0]$ & 0.08 \\
\hline Acyl-ghrelin & $6.2[4.6-6.3]$ & 8.9 [6.4-10.8] & 0.15 \\
\hline LPS & $78.0[77.7-79.1]$ & $78.9[72.7-86.5]$ & 1.00 \\
\hline LPS \& acyl-ghrelin & $76.2[70.5-82.2]$ & $75.3[74.2-76.8]$ & 0.77 \\
\hline
\end{tabular}

Table 3.12. Comparison of TNF $\alpha$ levels in different environments at 34 hours. Statistical analysis conducted using Kruskal-Wallis test. P-value less than 0.05 deemed significant and are in bold.

$\underline{I L-10}$

A hyperglycaemic environment was associated with a significant increase in IL-10 levels at both 17 and 34 hours within cell supernatants of vehicle treated mature adipocytes (Table 3.13 ) and only for acyl-ghrelin treated cells at 34 hours (Table 3.14). A hyperglycaemic environment demonstrated no significant effect on IL-10 levels at either time point when mature adipocytes were immuno-challenged with LPS. However, when LPS treatment was combined with acyl-ghrelin exposure there was a significant decline in IL-10 in a hyperglycaemic state at both 17 and 34 hours. 


\begin{tabular}{|c|c|c|c|}
\hline \multirow[b]{2}{*}{ Treatment } & \multicolumn{2}{|c|}{ IL-10 [pg/mL] } & \multirow[b]{2}{*}{ P-Value } \\
\hline & $\begin{array}{c}\text { Normoglycaemic } \\
\qquad(n=6)\end{array}$ & $\begin{array}{l}\text { Hyperglycaemic } \\
(n=6)\end{array}$ & \\
\hline Vehicle & $226.8( \pm 13.7)$ & $261.9( \pm 20.3)$ & 0.03 \\
\hline Acyl-ghrelin & $314.1( \pm 27.3)$ & $275.2( \pm 50.9)$ & 0.23 \\
\hline LPS & $264.3( \pm 26.6)$ & $234.6( \pm 34.3)$ & 0.22 \\
\hline LPS \& acyl-ghrelin & $292.3( \pm 19.5)$ & $140.0( \pm 23.4)$ & $<0.001$ \\
\hline
\end{tabular}

Table 3.13. Comparison of IL-10 levels in different environments at 17 hours. Statistical analysis conducted using an independent t-test. P value less than 0.05 deemed significant and are in bold.

\begin{tabular}{|c|c|c|c|}
\hline \multirow[b]{2}{*}{ Treatment } & \multicolumn{2}{|c|}{ IL-10 [pg/mL] } & \multirow[b]{2}{*}{ P-Value } \\
\hline & $\begin{array}{c}\text { Normoglycaemic } \\
(n=6)\end{array}$ & $\begin{array}{c}\text { Hyperglycaemic } \\
(n=6)\end{array}$ & \\
\hline Vehicle & $188.2( \pm 20.8)$ & $361.6( \pm 14.9)$ & $<0.001$ \\
\hline Acyl-ghrelin & $341.0( \pm 14.0)$ & $378.2( \pm 22.1)$ & 0.03 \\
\hline LPS & $196.4( \pm 17.5)$ & $178.4( \pm 17.5)$ & 0.19 \\
\hline LPS \& acyl-ghrelin & $303.9( \pm 25.2)$ & $173.1( \pm 14.9)$ & $<0.001$ \\
\hline
\end{tabular}

Table 3.14. Comparison of IL-10 levels in different environments at 34 hours. Statistical analysis conducted using an independent t-test. P-value less than 0.05 deemed significant and are in bold. 


\subsection{Discussion}

As this chapter focuses on the effect of acyl-ghrelin on lipid retention and inflammatory response, this discussion will be split into two sections; (i) acyl-ghrelin mediated lipid retention and (ii) acyl-ghrelin mediated inflammatory response.

\subsubsection{Acyl-ghrelin mediated lipid retention}

Exposure to acyl-ghrelin showed an increase in lipid retention within human mature adipocytes, this was seen in both a normo- and hyperglycaemic environment. These data correspond with previously published literature that has demonstrated acylghrelin promotes lipid accumulation in stromovascular fraction cells from omental tissue of obese normoglycaemic subjects (Rodriguez et al., 2009), murine mature adipocytes (3T3-L1) (Miegueu et al., 2011), male rat adipocytes (Muccioli et al., 2004) and in rat depot specific abdominal WAT (Davies et al., 2009). However, published data thus far only encompass a normoglycaemic environment, allowing this study to provide novel findings into the effect of acyl-ghrelin in hyperglycaemia on hypertrophy. When comparing glycaemic environments, it is evident that in normoglycaemia there is a gradual increase in lipid area upon exposure to acylghrelin, however in a hyperglycaemic environment there is a significant rise in lipid area as an immediate response to acyl-ghrelin treatment that remains unaltered over time. Thus, indicating that a hyperglycaemic environment is associated with a rapid acyl-ghrelin mediated response of lipid accumulation. 
In addition to an increased lipid area, acyl-ghrelin treatment demonstrated a role in the dysregulation of key lipid retention genes. Normoglycaemia showed an initial decrease in $A B C G 1$ expression, which was previously highlighted by Davies and colleagues, in rat abdominal WAT (Davies et al., 2009). This may be due to a delayed increase in $L X R B$ expression that has been shown to induce the expression of $A B C G 1$, counteracting acyl-ghrelin's effect (Maqdasy et al., 2016). However, these data do not correspond with previously published work that indicates both $A B C G 1$ and $L X R B$ expression are decreased in the presence of acyl-ghrelin (Davies et al., 2009). In contrast, a hyperglycaemic environment agreed with published literature indicating a marked decrease in ABCG1 and $L X R B$ expression. This could account for the immediate increase in cell lipid mass due to a resultant deficiency in the cellular export system. During both normoglycaemic and hyperglycaemic states, $\angle X R B$ and $A B C G 1$ were dysregulated in the presence of acyl-ghrelin, which could impede the removal of cellular lipids. $A B C G 1$ knockout mice have previously demonstrated the accumulation of cholesterol and phospholipids in macrophages (Kennedy et al., 2005). Evidence reported within this chapter demonstrates that acyl-ghrelin not only affects infiltrated macrophages in WAT but mature adipocytes themselves.

In agreement with other human mature adipocyte data (Rodriguez et al., 2009), SREBF1 expression was increased in the presence of acyl-ghrelin within a normoglycaemic state and continued to increase over time. This could either indicate the de novo synthesis of lipid droplets, or it suggests that SREBF1 is involved in regulating genes involved in cholesterol metabolism and counteracting acyl-ghrelin mediated lipid retention (Kersten, 2001, Wells, 2009). Further evidence 
regarding SREBF1 action shows that within a hyperglycaemic environment, expression remained unaltered in the presence of acyl-ghrelin, supporting data that show that a hyperglycaemic environment has diminished SREBF1 regulatory function allowing for a larger lipid area accumulation (Kersten, 2001, Wells, 2009).

When investigating the effect of the glycaemic environment, it was evident that hyperglycaemia caused a down regulation of lipid retention genes in both vehicle and acyl-ghrelin treatments. In humans, a study of lipid retention genes within obesity and patients with gestational diabetes showed a down regulation in $L X R$ and SREBF1 expression, thus demonstrating that transcriptional levels of the LXR-ABC pathway are dysregulated in the presence of hyperglycaemia (Lappas, 2014). Secondary to a hyperglycaemic effect on lipid genes, the LXR pathway may play a role in the progression of $T 2 \mathrm{D}$ via an effect on regulation of glucose homeostasis and insulin secretion (Maqdasy et al., 2016). GLUT4 is a key mediator in insulin induced glucose uptake (Tozzo et al., 1997) and is targeted by LXR, also its expression can be correlated with $A B C G 1$ expression in cultured human adipocytes (Dalen et al., 2003, Le Lay et al., 2001).

\subsubsection{Acyl-ghrelin mediated inflammatory response}

\section{Pro-inflammatory response}

Within normo- and hyperglycaemia environments, treatment with acyl-ghrelin on mature adipocytes exhibited a significant decline in IL-6 levels over time. Previous studies have confirmed a role for acyl-ghrelin in the attenuation of IL-6 release but 
only in the mucosa of colitic mice (Baatar et al., 2011), rat peritoneal macrophages (Chorny et al., 2008) and in plasma in acyl-ghrelin treated rats (Wu et al., 2008). LPS-stimulated release of IL-6 was not inhibited by acyl-ghrelin in normoglycaemia, however in a hyperglycaemic environment there was acyl-ghrelin-related inhibition of IL-6 levels. Wu and colleagues, reported similar findings within normoglycaemic male rats, where acyl-ghrelin did not directly inhibit cytokine release, such as IL-6, from LPS-stimulated cells (Wu et al., 2007). In contrast, within a normoglycaemic environment, LPS-treated human monocytes (Dixit et al., 2004) and mouse dopaminergic neurones (Beynon et al., 2013) were shown to exhibit an acyl-ghrelin inhibition of an LPS-induced increase of IL-6 levels, as only seen within human mature adipocytes in a hyperglycaemic state within this investigation.

TNF $\alpha$ data revealed no significant findings throughout this chapter, however further analysis of results indicate that all values were located at the lower end of the standard curve and obtained greater standard deviations to other experimental measures, therefore any statistical significance within this group would have been hard to elucidate. Previous studies have also reported that acyl-ghrelin effects on TNF $\alpha$ levels were not identified due to a lack of detection (Beynon et al., 2013). However, alternative sample sources have indicated that acyl-ghrelin does in fact supress TNF $\alpha$ levels with or without LPS induction (Baatar et al., 2011, Wu et al., 2007, Wu et al., 2008, Chorny et al., 2008, Dixit et al., 2004).

These findings highlight the relevant importance to the site-specific role acyl-ghrelin may play within an immune-compromised state in a pro-inflammatory response. Furthermore, the results enhance published evidence that acyl-ghrelin 
administration is shown to mediate protective effects in an LPS-mediated inflammatory state, especially in the presence of hyperglycaemia.

\section{Anti-inflammatory response}

The effects of acyl-ghrelin on IL-10 are contradictory. Acyl-ghrelin is predominantly reported to supress the response of IL-10 in T lymphocytes in mice (Xia et al., 2004, Hattori, 2009), however acyl-ghrelin has also been reported to increase IL-10 levels in mouse models (Gonzalez-Rey et al., 2006). Data presented in this chapter are also contradictory, finding that acyl-ghrelin significantly increased the level of IL-10 in a normoglycaemic environment, but exhibited no effect on IL-10 levels in a hyperglycaemic environment when compared to a vehicle. This could indicate that IL-10 levels within normoglycaemia are due to acyl-ghrelin promoting an antiinflammatory response in parallel to diminishing a pro-inflammatory response. Furthermore, I conclude that exposure to hyperglycaemia inhibits acyl-ghrelin's promotion of an anti-inflammatory response.

\subsection{Conclusion}

This chapter has presented relevant data to establish a role for acyl-ghrelin in the promotion of hypertrophy accompanied by the dysregulation of key lipid retention genes; $A B C G 1, \angle X R B$ and SREBF1. Furthermore, acyl-ghrelin has demonstrated a role in altering the immune response within human mature adipocytes in both normo- and hyperglycaemic environments. The prolonged effect of lipid export 
dysregulation is unknown and further in vivo work into acyl-ghrelin's effect in humans is required to establish whether an acyl-ghrelin mediated effect could have a downstream effect within T2D.

\subsection{Limitations}

Findings presented within this chapter were based on the culture of a human adipocyte cell line. As previously mentioned in section 2.1.1, primary adipocytes could also be utilized. The use of primary cells could have produced a more biologically relevant sample source, due to them being isolated directly from human tissue using either an enzymatic or mechanical method, however are notoriously difficult to culture and prone to contamination. Experimental design was developed to limit the phenotypical or morphological changes that can present within an infinite cell line and the protocol was successful in achieving this, however when comparing in vitro to ex vivo analysis this must be taken into consideration. 
Chapter 4

Exploration of acyl-ghrelin associated lipid

retention within ex vivo human visceral

adipose tissue 


\subsection{Introduction}

Visceral adipose tissue (VAT) is a blanket term used for adipose tissue located within the peritoneal cavity. In comparison to subcutaneous adipose tissue, VAT is often investigated in relation to metabolic disease due to visceral adipocytes having higher basal lipolysis, leaving it vulnerable to insulin resistance (Mårin et al., 1992). VAT is often split into five distinct types dependent upon the depot's anatomical location (Figure 4.1) (Mårin et al., 1992). Perirenal, gonadal (or surrounding the ovaries in females), pericardial, mesenteric and omental fat are all classed as visceral fat but they differ in metabolic function due to varying environmental stimuli in the distinct location in which they are found. Omental fat extends from the lower region of the stomach and encapsulates the abdomen (Lam et al., 2011). The accumulation of omental fat is associated with detrimental effects on an individual's health, thus, making it the primary source of visceral fat utilised for the study of obesity and related comorbidities (Lam et al., 2011, Matsuzawa, 2006, Maury et al., 2007). In vitro research on mature human adipocytes allows insight into adipose tissue function, however it does not consider that adipose tissue as an organ is comprised of a spectrum of cell types. Previous work involving ghrelin mediated lipid retention has predominantly focused on rodent epididymal fat (Davies et al., 2009). Findings indicated a relevant role for acyl-ghrelin and the model allowed for the ease of experimental design, however, it is likely to be limited in the translation to human pathophysiology due to the ever-evolving functionality of species-specific fat depots. 


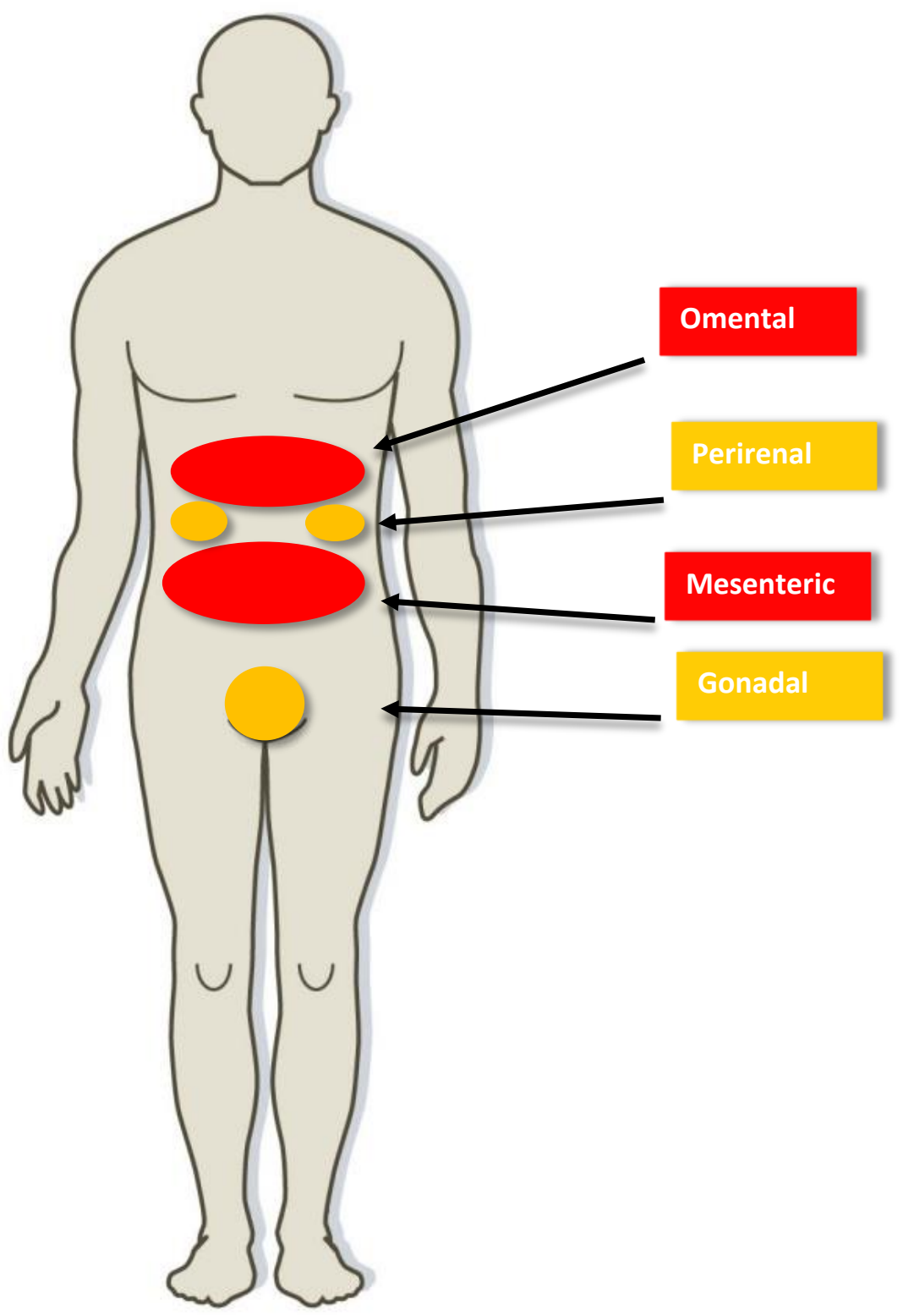

Figure 4.1. Visceral fat depots in the human body. Red colour indicates increased risk factor in metabolic disease. Orange colour indicates a lower risk level and red indicates higher risk associated with metabolic disease. 


\subsubsection{Adipose tissue, inflammation and oxidative stress}

The vast proportion of human adipose tissue is comprised of mature adipocytes, however it also contains pre-adipocytes (undifferentiated adipocytes), macrophages, monocytes, fibroblasts and a rich supply of blood vessels (Trujillo and Scherer, 2006). Adipose tissue within obesity results in a state of low grade inflammation, which is mediated and activated due to the infiltration of both proand anti-inflammatory macrophages (M1 and M2) (Shoelson et al., 2007). With obesity, adipose tissue macrophages (ATM) equate to $50 \%$ of all adipose tissue cells, in contrast to only $5 \%$ within lean individuals (Trujillo and Scherer, 2006). As previously discussed in Chapter 3, acyl-ghrelin can be associated with an increase of cellular lipid retention and altered immune response. Emerging evidence has linked the presence of GHSR-1 to M1 and M2 macrophages, which could alter adipose tissues inflammation via macrophage polarization (Lin et al., 2016). Adipose tissue contains numerous other immune cell types that can contribute towards inflammation and insulin resistance, for instance; dendritic cells, neutrophils, B cells and T cells.

Adipose tissue in obesity often has a dysregulated secretion of adipokines which has demonstrated a role in the pathogenesis of obesity-associated diseases such as T2D (Matsuzawa et al., 1999, Matsuda and Shimomura, 2013, Spiegelman and Flier, 2001). Obesity is also associated with increased formation of ROS (Fujita et al., 2006, Furukawa et al., 2017) and its subsequent harmful effects in promoting vascular (Stephens et al., 2009) and $\beta$-cell (Evans et al., 2003, Tiedge et al., 1997, Robertson et al., 2003) dysfunction, and ultimately diabetes (Stocker et al., 2007, Grattagliano et al., 2008). In vitro studies suggest that ROS generation is selectively 
increased in the fat tissues of obese mice which may facilitate insulin resistance and alter adipokine gene expression (Furukawa et al., 2017). A relationship for acylghrelin within the inflammatory state may be elucidated from the exploration of the effect of acyl-ghrelin on oxidative stress. Current literature demonstrated that increased plasma levels of acyl-ghrelin can be associated with a decrease in oxidative burden within obese subjects (Suematsu et al., 2005). Furthermore, evidence linking acyl-ghrelin to an increased adipose tissue mass may indicate a plausible role for acyl-ghrelin interaction within systemic oxidative stress, due to biomarkers of oxidative stress being correlated with fat accumulation (Matsuda and Shimomura, 2013, Keaney et al., 2003).

\subsubsection{Genes of interest}

Key lipid retention genes have been mentioned in detail (see Chapter 3 ) in relation to lipid profile; $L X R B, A B C G 1$ and SREBF1. Furthermore, within this chapter ghrelin axis gene expression will be examined to elucidate whether expression levels are altered as a result of transcriptional or translational effects of a hyperglycaemic environment. Desacyl-ghrelin is expressed in a prepro-ghrelin molecule and requires post translational octanoylation to produce the acyl-ghrelin complex. Investigation into transcriptional and translational causative agents are key for a further understanding of acyl-ghrelin effect, as to whether the variation in plasma levels of acyl-ghrelin is due to changes in ghrelin (GHRL) gene expression or the presence of key octanoylation genes i.e. LYPLA1 and mBOAT4 (Satou et al., 2010, Kirchner et al., 2012). 


\subsection{Aim}

To expand on the outcomes gained in Chapter 3, the goal was to translate the in vitro results into an ex vivo system, allowing for the comparison of a local versus global effect. I hypothesis this would result in evidence providing an association between circulatory acyl-ghrelin levels with lipid retention gene and circulatory lipid profiles. Furthermore, in line with Chaper 3 I envisaged that acyl-ghrelin levels would demonstrate a positive association with anti-inflammatory markers and a negative association with pro-inflammatory markers. Therefore, this chapter aims to:-

1. Evaluate plasma acyl-ghrelin levels in relation to the ghrelin gene axis within hVAT

2. Investigate the expression levels of lipid retention genes within hVAT

3. Investigate levels of circulating inflammatory marker within cohorts

4. Explore the association of lipid retention genes and inflammatory markers with acyl-ghrelin levels 


\subsection{Methods}

\subsubsection{Sample collection}

Human visceral adipose tissue biopsies, were collected as described in detail in Section 2.2.1. Briefly, 30 participants, 10 per group for non-obese (NO), obese (O) and obese with T2D (OT2D).

\subsubsection{Baseline characteristics}

To establish participant's metabolic state, key analytical chemistry including lipid and glucose profiles, were analysed from fasted plasma samples from each participant. Analysis of glucose, cholesterol, HDL, albumin, creatinine and triglycerides was undertaken using a Randox Daytona plus, with LDL concentration calculated using the Friedewald equation as discussed in Section 2.2.6.1. Determination of $\mathrm{HbA1C}$ was done using the BioRad D-10 haemoglobin analyser, as discussed in Section 2.2.6.1. All other data was acquired from patient hospital notes and was correct at the time of operation.

\subsubsection{Quantitative Real Time PCR}

Real Time-PCR was performed on 30 hVAT, which included samples from participants classed as non-obese $(N O, n=10)$, obese $(O, n=10)$ and obese with T2D (OT2D, n=10). RNA was extracted from the hVAT as previously described (Section

2.2.5) and reverse transcribed to CDNA (Section 2.3.1.2). Specific primers were designed and synthesised either by Eurofins MWG Operon or were purchased from PrimerDesign as previously mentioned (Section 2.3.3.2). The primer sequences were run on a primer specific amplification programme dependent upon annealing 
temperatures (Appendix 2). Optimum primer conditions required a primer efficiency of $90-110 \%$ and a standard curve $\mathrm{R}^{2}$ value greater than 0.99 , primer validation confirmed for all primer sets (Appendix 3).

\subsubsection{Gene expression analysis}

Threshold cycles $(\mathrm{Ct})$ were accepted under the following criteria; single melt peak, triplicates within $1 \mathrm{Ct}$ of each other and valid control values. The average $\mathrm{Ct}$ was taken per sample and normalised against the amplification of b-actin. Gene expression data are presented as fold change and deduced using the $2^{-\Delta \Delta \mathrm{Ct}}$ method as discussed in Section 2.3.3.4, raw data are available in Appendix 6.

\subsubsection{ELISA}

A human ghrelin (active) ELISA (Merck Millipore ${ }^{\mathrm{TM}}$ ) was used to specifically determine fasting plasma acyl-ghrelin levels in plasma samples collected at the time of surgery and treated the with protease inhibitor AESBF $(2 \mathrm{mg} / \mathrm{mL})$. Plasma cytokine levels were determined using ELISA for IL-6, IL-10 and TNFa. All reagents were included in the ELISA kits and the protocols run following the manufacturers guidelines as described in Section 2.4.

\subsubsection{Oxidative stress measurement}

Oxidative stress was measured via the analysis of patient's fasting plasma using total antioxidant status (TAOS) assay as previously described in detail in Section 2.2.8. 


\subsubsection{Data analysis}

Statistical analysis was conducted on raw data using SPSS version 22 and test procedures were dependent on normality testing, as described in Section 2.5. Continuous variables included all variables except for medication which was treated as a categorical variable and treated as described in Section 2.5.1.

\subsection{Results}

Samples were split into three distinct arms for data analysis to enable the investigation of several factors:

(i) Total sample cohort (NO v O v OT2D)

(ii) Diabetes effect $(\mathrm{NO}+\mathrm{O})$ v OT2D)

(iii) Obesity effect (O+OT2D) v NO)

\subsubsection{Total sample cohort}

\subsubsection{Baseline characteristics}

The baseline characteristics of the total sample cohort were well matched in terms of age. However, as expected, there were significant differences between measurements for weight, BMI, glucose and HbA1c (Table 4.1). 


\begin{tabular}{|c|c|c|c|c|}
\hline & $\begin{array}{c}\text { Non Obese } \\
(\mathrm{NO}) \\
(\mathrm{n}=10)\end{array}$ & $\begin{array}{c}\text { Obese } \\
(\mathrm{O}) \\
(\mathrm{n}=10)\end{array}$ & $\begin{array}{c}\text { Obese Type 2 } \\
\text { (OT2D) } \\
(\mathrm{n}=10)\end{array}$ & P-value \\
\hline Age (Years) & $51.8(15.5)$ & $51.1(12.0)$ & $45.5(6.8)$ & 0.44 \\
\hline Weight (Kg)* & $72.2[64-81]$ & $90.7[86-122]$ & $131.4[114-152]$ & $<0.001$ \\
\hline \begin{tabular}{c} 
BMI (Kg/m $)^{*}$ \\
\hline Glucose
\end{tabular} & $26.2[24-28]$ & $34.9[32-42]$ & $47.3[42-51]$ & $<0.001$ \\
\hline $\begin{array}{c}\text { (mmol/L)* } \\
\text { HbA1c }\end{array}$ & $3.8[4.5-6.5]$ & $5.4[4.9-6.5]$ & $6.7[6.0-12.6]$ & $<0.01$ \\
\hline $\begin{array}{c}\text { (mmol/mol)* } \\
\text { prescription } \\
\text { (\%) }\end{array}$ & $30.6[26.3-$ & $36.1[30.3-$ & $52.5[35.8-68.1]$ & $<0.05$ \\
\hline $\begin{array}{c}\text { HbA1c (\%)* } \\
\text { Statin }\end{array}$ & $5.1[4.6-5.7]$ & $5.2[4.9-5.5]$ & $7.0[5.4-8.4]$ & $<0.05$ \\
\hline
\end{tabular}

Table 4.1 Baseline characteristics for total cohort. Mean and standard deviation shown for normally distributed data \& p-value determined using one way ANOVA. * Median and interquartile range shown for data that is not normally distributed and Kruskal Wallis used for $p$ val determination. ${ }^{\#}$ Categorical data tested using Pearson Chi-square analysis, \% shown with $\mathrm{n}$ in brackets. Significant $\mathrm{p}$ value are shown in bold.

\subsubsection{Plasma levels of acyl-ghrelin}

Acyl-ghrelin levels were measured in fasting plasma samples of all 30 participants. Analysis indicates a significantly decreased circulating level of acyl-ghrelin present within obese individuals with T2D in comparison to both obese (OT2D v 0; 228.5 [98.4-439.4] v 515.5 [308.7-701.2] pg/mL; $\mathrm{p}<0.05$ ) and non-obese individuals (OT2D 
v NO; 228.5 [98.4-439.4] v 467.2 [325.7-508.3] pg/mL; $p<0.05)$. However, no significant difference was seen when the non-obese and obese cohorts were compared $(p=0.71)$ (Figure 4.2). Fasting acyl-ghrelin was negatively correlated with fasting plasma glucose levels $\left(r_{s}=-0.37, p<0.05\right)$ and body weight $\left(r_{s}=-0.42, p<0.05\right)$, respectively across the total sample cohort (Figure 4.3A-B).

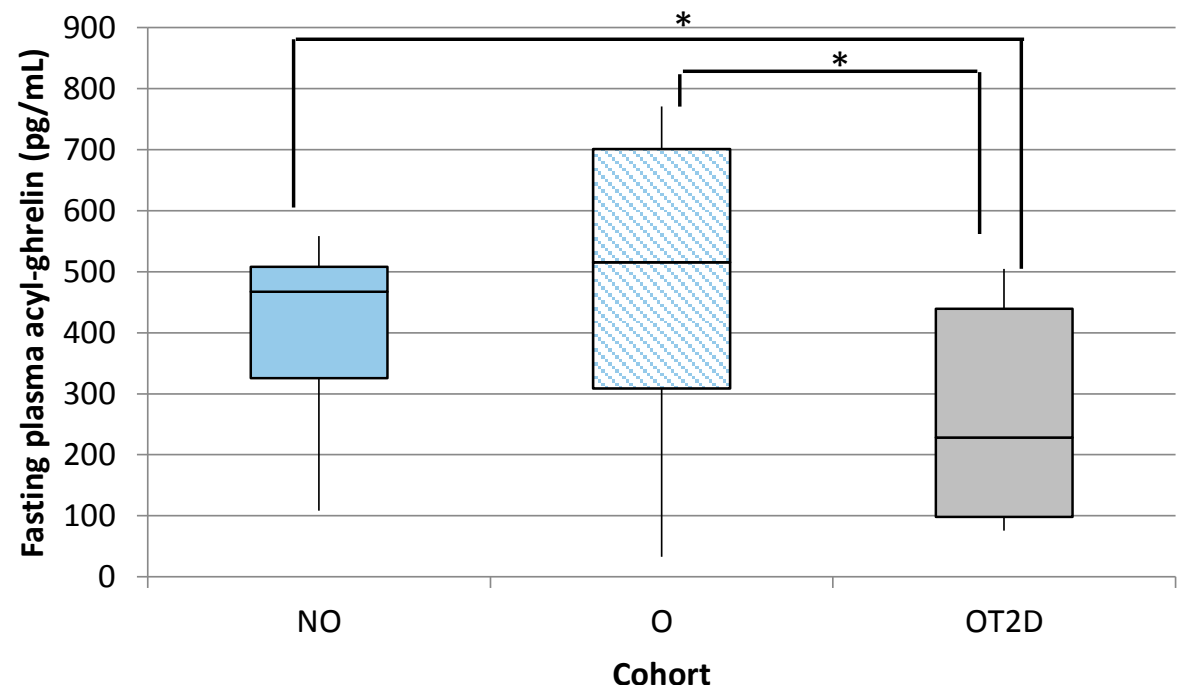

Figure 4.2. Box plot of fasting acyl-ghrelin levels within the three cohorts. Median acyl-ghrelin levels shown for non-obese (NO), obese (O) and obese type 2 (OT2D)( $n=10$ per cohort). Statistical analysis was conducted using non-parametric measures, Kruskal Wallis determined statistical significance $(p<0.05)$ between groups which are indicated by *

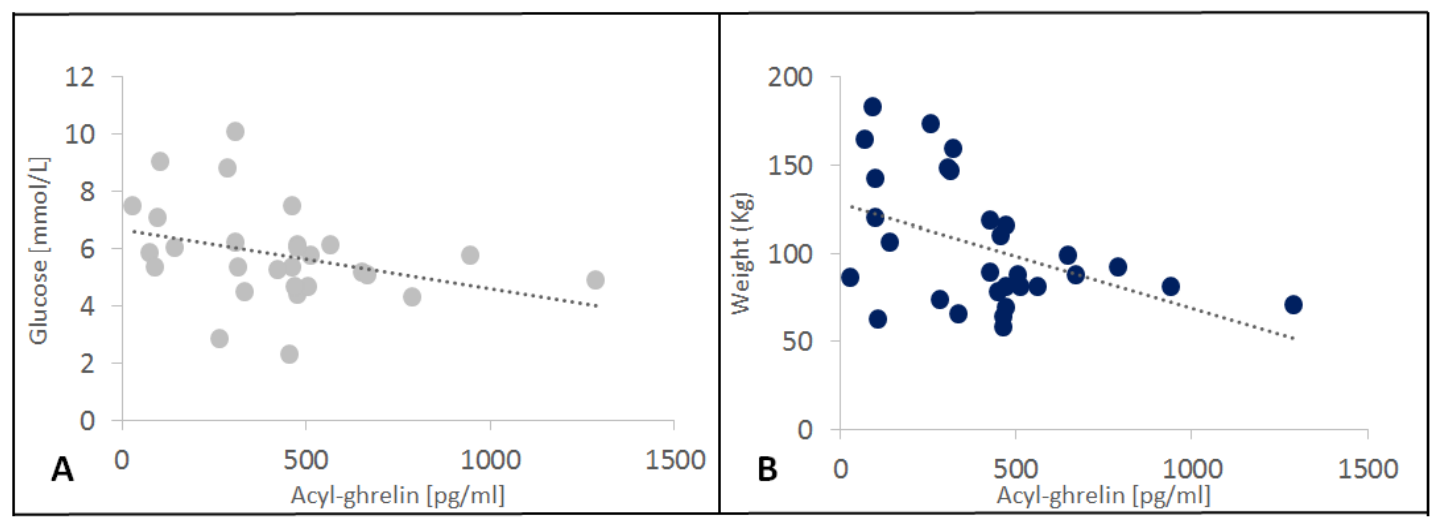

Figure 4.3A-B. Scatter graphs between acyl-ghrelin and glucose and acyl-ghrelin and body weight. APlasma glucose $\left(r_{s}=-0.414 ; p<0.05\right), y=-0.002 x+6.6695, R^{2}=0.1129(n=30)$. B- Body weight $\left(r_{s}=-\right.$ $0.421 ; p<0.05), y=-0.059 x+128.15, R^{2}=0.2007(n=30)$. 


\section{Ghrelin gene axis expression across cohorts}

Gene expression fold change data produced from the total sample cohort indicates that across groups there was no significant change in gene expression for mBOAT4 or LYPLA1. Ghrelin gene expression was significantly down regulated, with a 2.6fold change within the OT2D group compared to the $O$ group. However, fold change data signified no alteration between OT2D when compared to NO, despite a significant increase in GHRL $\Delta$ Ct values (Table 4.2).

\section{Plasma acyl-ghrelin levels in relation to ghrelin gene axis}

Plasma acyl-ghrelin levels were analysed with respect to its relationship with key octanoylation genes (Table 4.3). The data indicates that within the total sample cohort circulating acyl-ghrelin levels were associated with the $\Delta \mathrm{Ct}$ values of $G H R L$ expression $(p<0.05)$, however there was no associations between circulating acylghrelin levels and LYPLA1 $(p=0.620)$ or mBOAT4 $(p=0.587)$. 


\begin{tabular}{|c|c|c|c|c|}
\hline Group & Gene of interest & Fold Change & Direction & P-value \\
\hline \multirow{3}{*}{ NO v O } & $G H R L$ & 1.50 & $\longleftrightarrow$ & 0.19 \\
\hline & mBOAT4 & 0.88 & $\longleftrightarrow$ & 0.42 \\
\hline & LYPLA1 & 1.45 & $\longleftrightarrow$ & 0.17 \\
\hline \multirow{3}{*}{ NO v OT2D } & GHRL & 0.58 & $\longleftrightarrow$ & $<0.05$ \\
\hline & mBOAT4 & 0.66 & $\longleftrightarrow$ & 0.22 \\
\hline & LYPLA1 & 1.13 & $\longleftrightarrow$ & 0.42 \\
\hline \multirow{3}{*}{ O v OT2D } & GHRL & 2.60 & 1 & $<0.05$ \\
\hline & mBOAT4 & 0.75 & $\longleftrightarrow$ & 0.41 \\
\hline & LYPLA1 & 0.78 & & 0.28 \\
\hline
\end{tabular}

Table 4.2. Relative fold change values for ghrelin axis gene expression data for total cohort. P-value deduced from $\Delta \mathrm{Ct}$ T-Test. Significant values shown in bold. $\mathrm{N}=10$ per cohort.

$\downarrow=$ down regulation $\leftrightarrow=$ no overall change

\begin{tabular}{|l|l|l|}
\hline \multicolumn{2}{|l|}{} & $\begin{array}{l}\text { Plasma } \\
\text { acyl-ghrelin }\end{array}$ \\
\hline \multirow{2}{*}{ GHRL } & Spearman's Correlation & $\begin{array}{l}\mathbf{0 . 4 0 8} \\
\mathbf{0 . 0 3 1}\end{array}$ \\
\hline \multirow{2}{*}{ LYPLA1 } & P-value & -0.098 \\
& Spearman's Correlation & 0.620 \\
\hline \multirow{2}{*}{ MBOAT4 } & P-value & 0.107 \\
& Spearman's Correlation & 0.587 \\
\hline
\end{tabular}

Table 4.3 Correlation data for gene expression and plasma acyl-ghrelin for total cohort. Data analysis of relative $\Delta C t$ value and raw plasma marker levels $(n=30) . \Delta C t$ values are inverse on gene transcript level. Non-parametric data analysis via Spearman's rho correlation used to determine correlation between parameters. Significant values shown in bold. 


\subsubsection{Lipid profiles}

Lipid profiles were analysed across the total sample cohort, showing significant differences in total cholesterol $(p<0.05), \mathrm{HDL}(p<0.05)$ and LDL $(p<0.01)$ levels. However, triglyceride concentrations did not differ between groups $(p=0.19)$ (Table 4.4). Further analysis showed that there were no significant differences within lipid marker levels between NO and O. Upon separate comparison of both the NO and O groups to the OT2D group, there was a significant decline in total cholesterol $(p<0.05), H D L(p<0.01)$ and LDL $(p<0.01)$ levels, with a significant increase in TGs within OT2D compared to NO $(p<0.05)$. Lipid profiles were analysed to assess any associations between circulating acyl-ghrelin and lipid markers, which indicated positive correlations for total cholesterol $\left(r_{s}=0.39, p<0.05\right)$ and LDL $\left(r_{s}=0.39\right.$, $\mathrm{p}<0.05$ ), respectively (Figure 4.4A-B).

\begin{tabular}{|c|c|c|c|c|}
\hline & $\begin{array}{c}\text { Non-Obese } \\
(\mathrm{NO}) \\
(\mathrm{n}=10)\end{array}$ & $\begin{array}{c}\text { Obese } \\
(\mathrm{O}) \\
(\mathrm{n}=10)\end{array}$ & $\begin{array}{c}\text { Obese Type } \\
\text { (OT2D) } \\
(\mathrm{n}=10)\end{array}$ & P-value \\
\hline Cholesterol (mmol/L) & $4.4(1.2)$ & $4.8(1.7)$ & $3.1(0.7)$ & $<0.05$ \\
\hline $\mathrm{HDL}(\mathrm{mmol} / \mathrm{L})$ & $1.1(0.4)$ & $1.3(0.8)$ & $0.7(0.2)$ & $<0.05$ \\
\hline LDL (mmol/L) & $2.7(0.9)$ & $2.8(1.1)$ & $1.5(0.5)$ & $<0.01$ \\
\hline Triglycerides & $1.2(1.0)$ & $1.5(0.6)$ & $1.9(0.8)$ & 0.19 \\
\hline (mmol/L) & & & & \\
\hline
\end{tabular}

Table 4.4. Lipid profiles for total cohort. Mean and standard deviation shown for normally distributed data \& p-value determined using one way ANOVA. Significant values are shown in bold. 


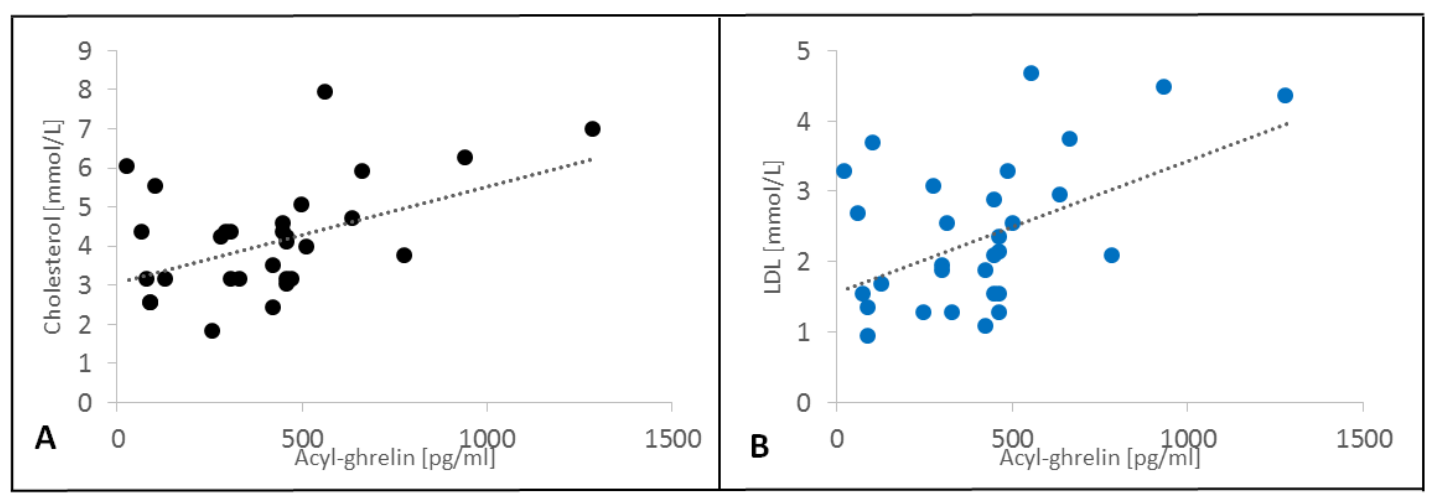

Figure 4.4A-B. Scatter graphs between acyl-ghrelin and cholesterol and acyl-ghrelin and LDL. APlasma total cholesterol $\left(r_{s}=0.380 ; p<0.05\right) . y=0.0025 x+3.0373, R^{2}=0.2301(n=30)$. B- Plasma low density lipoprotein $(L D L)\left(r_{s}=0.393 ; p<0.05\right) y=0.0019 x+1.5463, R^{2}=0.2437(n=30)$.

\section{Lipid retention gene expression across cohorts}

The total sample cohort indicated there was no significant change in the overall gene expression of lipid retention genes when the $O$ group was compared to the NO group. However, when the OT2D group was compared to both the $\mathrm{O}$ and NO groups, there was a marked decrease in $L X R B, A B C G 1$ and SREBF1 (Table 4.5).

\section{Plasma acyl-ghrelin levels in relation to lipid retention genes}

When lipid retention gene expression was analysed against plasma acyl-ghrelin levels, there was a significant negative correlation between acyl-ghrelin and ABCG1 $\Delta C t$, with high acyl-ghrelin correlating to an increase in $A B C G 1$ expression $\left(r_{s}=-0.39\right.$, $\mathrm{p}<0.05)$. LXRB was shown to have a similar relationship with acyl-ghrelin levels as $A B C G 1$, however this was non-significant $\left(r_{s}=-0.36, p=0.06\right)$. In addition to plasma acyl-ghrelin, GHRL expression also produced a significant association with plasma lipid levels which indicated a negative correlation between ghrelin gene $\Delta \mathrm{Ct}$ values versus total cholesterol $\left(r_{s}=-0.54, p<0.01\right)$ and LDL $\left(r_{s}=-0.49, p<0.01\right)$, respectively. 


\begin{tabular}{|c|c|c|c|c|}
\hline Group & Gene of interest & Fold Change & Direction & P-value \\
\hline \multirow{4}{*}{ NO v O } & LXRB & 0.73 & 4 & 0.16 \\
\hline & $A B C G 1$ & 0.84 & $\longleftrightarrow$ & 0.24 \\
\hline & SREBF1 & 1.12 & $\longleftarrow$ & 0.39 \\
\hline & PPARY & 0.73 & $\longleftrightarrow$ & 0.22 \\
\hline \multirow{4}{*}{ NO v OT2D } & LXRB & 2.78 & | & 0.02 \\
\hline & ABCG1 & 2.44 & 1 & $<0.001$ \\
\hline & SREBF1 & 2.70 & $\mid$ & $<0.05$ \\
\hline & PPARY & 0.66 & $\longleftarrow$ & 0.27 \\
\hline \multirow{4}{*}{ O v OT2D } & LXRB & 2.04 & 1 & $<0.05$ \\
\hline & ABCG1 & 2.04 & 1 & $<0.05$ \\
\hline & SREBF1 & 3.03 & & $<0.001$ \\
\hline & PPARY & 0.91 & & 0.32 \\
\hline
\end{tabular}

Table 4.5. Relative fold change values for lipid retention gene expression data for total cohort. Pvalue deduced from $\Delta \mathrm{Ct}$ T-Test. Significant values are shown bold. $\mathrm{N}=10$ per cohort.

$\downarrow=$ down regulation $\leftrightarrow$ = no overall change

\subsubsection{Inflammation markers}

\section{Plasma cytokine levels}

Inflammatory cytokines were analysed across the total sample cohort (NO, O and OT2D) using non-parametric parameters. The results indicated a non- significant alteration in levels of IL- 6 and TNF $\alpha$ between the three groups, however there was a significant difference in IL-10 levels (Table 4.6). Analysis demonstrated that NO v O produced no difference in IL-6 and TNF $\alpha$ (IL-6; $12.9 \vee 11.0$ pg/mL; $p=0.71$. TNF $\alpha$; 
$11.4 \vee 36.0 \mathrm{pg} / \mathrm{mL} ; \mathrm{p}=0.26$, respectively), however, IL-10 levels were significantly reduced within the $\mathrm{O}$ group when compared to $\mathrm{NO}(88.6 \vee 53.5 \mathrm{pg} / \mathrm{mL}, \mathrm{p}<0.05)$. Results were mirrored when comparing NO $\vee$ OT2D groups, which indicated a marked decrease in IL-10 (88.6 v $39.6 \mathrm{pg} / \mathrm{mL}, \mathrm{p}<0.05)$ and unaltered levels of IL-6 $(12.9 \vee 7.9, p=0.60)$ and TNF $\alpha(11.4 \vee 20.1 \mathrm{pg} / \mathrm{mL}, \mathrm{p}=0.47)$. However, results between $O \vee$ OT2D indicated that IL-6 ( $p=0.31)$, TNF $\alpha(p=0.37)$ and IL-10 ( $p=0.14)$ levels were all similar between the groups.

\begin{tabular}{|c|c|c|c|c|}
\hline $\begin{array}{c}\text { Cytokine } \\
{[\mathrm{pg} / \mathrm{mL}]}\end{array}$ & $\begin{array}{c}\text { Non-Obese } \\
(\mathrm{NO}) \\
(\mathrm{n}=10\end{array}$ & $\begin{array}{c}\text { Obese } \\
(\mathrm{O}) \\
(\mathrm{n}=10)\end{array}$ & $\begin{array}{c}\text { Obese Type 2 } \\
(\mathrm{OT2D}) \\
(\mathrm{n}=10)\end{array}$ & P-value \\
\hline IL-6 & 12.9 & 11.0 & 7.9 & 0.61 \\
\hline TNF & 11.4 & 36.0 & 20.1 & 0.42 \\
& {$[5.1-43.4]$} & {$[17.7-54.0]$} & {$[14.0-32.6]$} & \\
\hline IL-10 & 88.6 & 53.5 & 39.6 & $<0.05$ \\
& {$[66.3-129.5]$} & {$[27.5-67.0]$} & {$[44.2-15.1]$} & \\
\hline
\end{tabular}

Table 4.6. Cytokine levels for total cohort. Median and interquartile range for cytokine levels for each cohort. P-value determined via Kruskal-Wallis across global data. Significant values shown in bold.

\section{$\underline{\text { Total Antioxidant Status }}$}

Data analysis of the total sample cohort showed a significant difference in overall TAOS (\%) between groups (NO $\vee \mathrm{O} \vee$ OT2D; $53.5 \% \vee 44.2 \% \vee 31.1 \% ; p<0.05$, respectively). Post hoc analysis of data demonstrated that TAOS levels present in 
the OT2D cohort were significantly lower than both the $O$ and NO groups $(p<0.01$, $p<0.001$, respectively) (Figure 4.5).

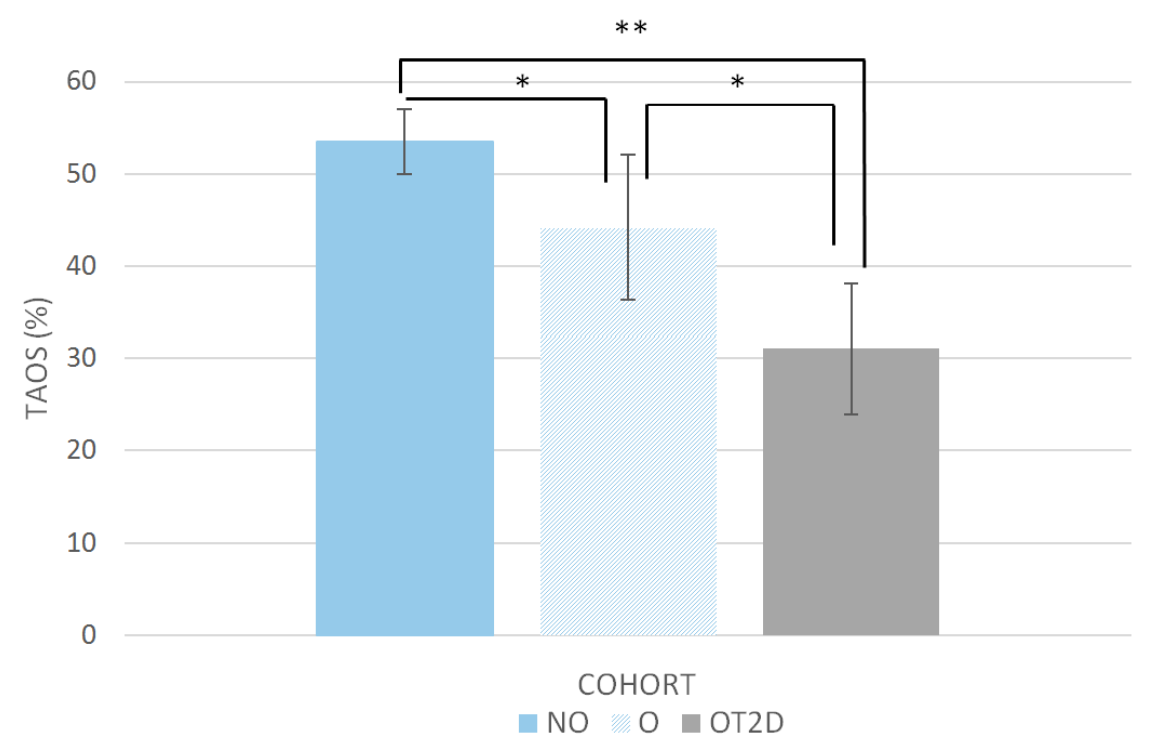

Figure 4.5. Bar graph of mean TAOS (\%) within the three cohorts. Statistical analysis was conducted using a one-way ANOVA and post hoc Tukey. Statistical significance between groups are indicated by asterisks $(* p<0.01 \& * * p<0.001)(n=10$ per cohort $)$.

\section{Inflammation vs acyl-ghrelin}

Total sample cohort cytokine data did not show a significant association between any inflammatory markers and plasma acyl-ghrelin (IL-6, $p=0.163 ;$ TNF $\alpha, p=0.957$; IL-10, $p=0.633$; TAOS, $p=0.190)$. However, when inflammatory markers were analysed against GHRL expression it showed that an increase in GHRL expression was associated with an increase in plasma IL-10 (GHRL $\Delta$ Ct vs IL10; $p<0.05$ ) and TAOS \% (GHRL $\triangle$ Ct vs TAOS \%; p<0.05) (Table 4.7). 


\begin{tabular}{|l|l|l|l|}
\hline \multicolumn{2}{|l|}{} & $\begin{array}{l}\text { Plasma } \\
\text { acyl-ghrelin }\end{array}$ & $\begin{array}{l}G H R L \\
\Delta \mathrm{Ct}\end{array}$ \\
\hline IL-6 & Spearman's Correlation & -0.276 & 0.054 \\
& P value & 0.163 & 0.785 \\
\hline TNF $\alpha$ & Spearman's Correlation & 0.015 & 0.440 \\
& P value & 0.957 & 0.060 \\
\hline IL-10 & Spearman's Correlation & 0.129 & $\mathbf{- 0 . 4 7 6}$ \\
& P value & 0.633 & $\mathbf{0 . 0 4 4}$ \\
\hline \multirow{2}{*}{ TAOS \% } & Spearman's Correlation & 0.255 & $\mathbf{- 0 . 3 9 6}$ \\
& P value & 0.190 & $\mathbf{0 . 0 3 0 *}$ \\
\hline
\end{tabular}

Table 4.7 Correlation data for gene expression, plasma acyl-ghrelin and inflammatory markers for total cohort. Data analysis of relative $\Delta C t$ value and raw plasma marker levels $(n=30) . \Delta C t$ values are inverse on gene transcript level. Non-parametric data analysis via Spearman's rho correlation used to determine correlation between parameters. Significant values were shown in bold.

\subsubsection{Diabetes effect}

The second arm of data analysis, aimed to investigate the effect of diabetes (No Diabetes $(\mathrm{NO}+\mathrm{O})) \vee$ Diabetes $(\mathrm{OT} 2 \mathrm{D}))$. To establish the effect of diabetes on the association of key variables, correlation analysis was conducted for samples in the OT2D group only.

\subsubsection{Baseline characteristics}

Comparison of baseline characteristics demonstrated no significant differences in participant's ages. However, as expected, there was a significant increase in weight, BMI, fasting blood glucose and HbA1c in those with diabetes (Table 4.8). 


\begin{tabular}{|c|c|c|c|}
\hline & $\begin{array}{c}\text { No Diabetes } \\
(\mathrm{NO}+\mathrm{O}) \\
(\mathrm{n}=20)\end{array}$ & $\begin{array}{c}\text { Diabetes } \\
\text { (OT2D) } \\
(\mathrm{n}=10)\end{array}$ & P-value \\
\hline Age (Years) & $51.5(13.5)$ & $45.4(6.8)$ & 0.20 \\
\hline Weight (Kg)* & $82.0[72-10]$ & $131.4[116-148]$ & $<0.01$ \\
\hline BMI (Kg/m $\left.{ }^{2}\right)^{*}$ & $29.9[26-35]$ & $47.3[43-50]$ & $<0.001$ \\
\hline Glucose (mmol/L)* & $5.3[4.6-5.9]$ & $6.7[6.0-11.1]$ & $<0.01$ \\
\hline HbA1c (\%)* & $5.2[4.6-5.6]$ & $7.0[5.5-7.6]$ & $<0.01$ \\
\hline HbA1c (mmol/mol)* & $33.3[26.8-37.4]$ & $52.5[35.8-68.1]$ & $<0.01$ \\
\hline Statin prescription \\
(\%) & $15(3)$ & $50(5)$ & $<0.05$ \\
\hline
\end{tabular}

Table 4.8. Baseline characteristics of the 'No diabetes', and 'Diabetes' cohorts. Mean and standard deviation shown for normally distributed data \& p-value determined using independent t-test.

* Median and interquartile ranges shown for not normally distributed data and Kruskal Wallis used for $\mathrm{p}$ value determination. "Categorical data tested using Pearson Chi-square analysis, \% shown with $\mathrm{n}$ in brackets. Significant values shown in bold.

\subsubsection{Plasma levels of acyl-ghrelin}

Fasting plasma acyl-ghrelin levels were significantly lower within those with T2D (OT2D) when compared to those without T2D (NO + O) (228.5 [98.4-439.4] v 464.8 [314.0 - 528.7] pg/mL; $p<0.05)$ (Figure 4.6). 


\section{Ghrelin gene axis expression across cohorts}

Gene expression analysis for the ghrelin gene axis indicates that within the diabetes group (OT2D) there was no significant difference in gene expression for mBOAT4 or LYPLA1. However, GHRL expression itself was significantly down regulated at a 2.1fold change within the OT2D group in comparison to the no diabetes group $(p<0.05)$ (Table 4.9).

\section{Plasma acyl-ghrelin levels in relation to ghrelin gene axis}

Plasma acyl-ghrelin levels within the diabetes group were not significantly associated with the key octanoylation genes LYPLA1 $(p=0.150)$ or mBOAT4 $(p=0.144)$ (Table 4.10). Data indicated that within the diabetes group circulating acyl-ghrelin levels were negatively associated with the $\Delta C$ t values of $G H R L$ expression $(p<0.05)$

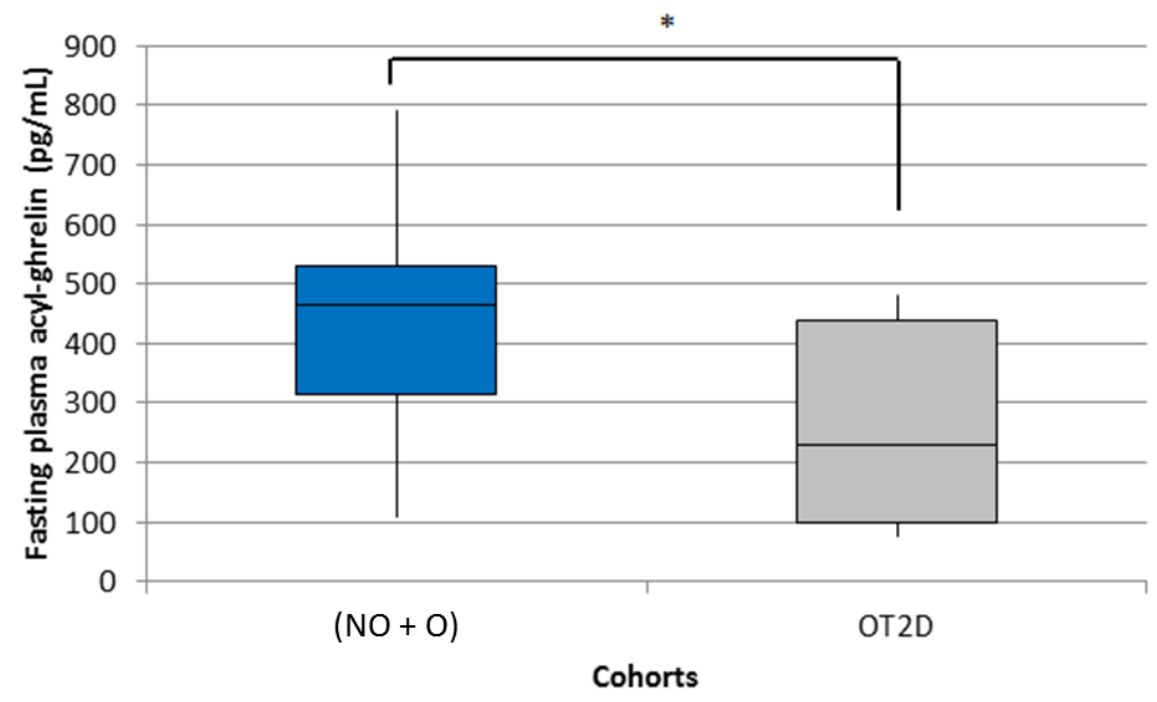

Figure 4.6. Box plot of fasting acyl-ghrelin levels for 'No diabetes' and 'Diabetes' cohorts. Median acyl-ghrelin levels shown for no diabetes $(\mathrm{NO}+\mathrm{O})(\mathrm{n}=20)$ and those with Type 2 diabetes $(O T 2 D)(n=10)$. Statistical analysis was conducted using non-parametric measures, independent sample t-test determined statistical significance $(\mathrm{p}<0.05)$ between groups which are indicated by *. 


\begin{tabular}{|c|c|c|c|c|}
\hline Group & Gene of interest & Fold Change & Direction & P-value \\
\hline \multirow{3}{*}{ (NO + O) $v$} & GHRL & $\mathbf{2 . 1 0}$ & $\downarrow$ & $<0.05$ \\
\cline { 2 - 5 } OT2D & mBOAT4 & 0.71 & $\longleftarrow$ & 0.26 \\
\cline { 2 - 5 } & LYPLA1 & 0.94 & $\longleftrightarrow$ & 0.28 \\
\hline
\end{tabular}

Table 4.9. Relative fold change values for ghrelin axis gene expression data for diabetes effect cohort. P-value deduced from $\Delta$ Ct T-Test. Significant values shown in bold.

$$
\downarrow \text { = down regulation } \leftrightarrow=\text { no overall change }
$$

\begin{tabular}{|l|l|l|}
\hline \multicolumn{2}{|l|}{} & $\begin{array}{l}\text { Plasma } \\
\text { acyl-ghrelin }\end{array}$ \\
\hline GHRL & Spearman's Correlation & $\mathbf{- 0 . 7 9 1}$ \\
& P-value & $\mathbf{0 . 0 1 0 *}$ \\
\hline LYPLA1 & Spearman's Correlation & 0.150 \\
& P-value & 0.680 \\
\hline mBOAT4 & Spearman's Correlation & 0.144 \\
& P-value & 0.691 \\
\hline
\end{tabular}

Table 4.10 Correlation data for gene expression and plasma acyl-ghrelin for diabetes effect cohort. Data analysis of relative $\Delta C t$ value and raw plasma marker levels $(n=10) . \Delta C t$ values are inverse on gene transcript level. Non-parametric data analysis via Spearman's rho correlation used to determine correlation between parameters. Significant values shown in bold.

\subsubsection{Lipid profiles}

Lipid profiles were analysed across the two groups, showing a significant difference in plasma lipid markers. Significant differences were demonstrated in total cholesterol $(p<0.01), \mathrm{HDL}(p<0.05)$ and LDL $(<0.01)$ levels, however triglyceride profiles remained unaltered across the groups $(p=0.10)$ (Table 4.11). Due to these data being opposed to the normal distribution of data expected to be seen within this group of individuals, lipid profiles were further assessed in relation to lipid 
management drugs. However, this analysis deduced there was no significant variation within the groups lipid profiles between those prescribed statins $(n=5)$ and those who were not $(n=5)$ (cholesterol; $p=0.202, \mathrm{HDL} ; p=0.141, \mathrm{LDL} ; \mathrm{p}=0.246$ and triglycerides; $p=0.794)$.

\begin{tabular}{|c|c|c|c|}
\hline & $\begin{array}{c}\text { No Diabetes } \\
(\mathrm{NO}+\mathrm{O}) \\
(\mathrm{n}=20)\end{array}$ & $\begin{array}{c}\text { Diabetes } \\
\text { (OT2D) } \\
(\mathrm{n}=10)\end{array}$ & P-value \\
\hline Cholesterol (mmol/L) & $4.6(1.4)$ & $3.1(0.7)$ & $<0.01$ \\
\hline $\mathrm{HDL} \mathrm{(mmol/L)}$ & $1.2(0.6)$ & $0.7(0.2)$ & $<0.05$ \\
\hline LDL (mmol/L) & $2.7(1.0)$ & $1.5(0.5)$ & $<0.01$ \\
\hline Triglycerides (mmol/L) & $1.4(0.8)$ & $1.9(0.8)$ & 0.10 \\
\hline
\end{tabular}

Table 4.11. Lipid profiles for diabetes effect cohort. Mean and standard deviation shown for normally distributed data \& p-value determined using an independent T-Test. Significant values shown in bold.

\section{Lipid retention gene expression across cohorts}

Gene expression data for genes involved in lipid retention were analysed to produce fold change with respect to a diabetes effect $((\mathrm{O}+\mathrm{NO}) \vee \mathrm{OT} 2 \mathrm{D})$. No diabetes compared to diabetes expression patterns mirrored that of the total sample cohort, showing a significant down regulation of key genes involved in lipid retention, LXRB $(p<0.05)$ and $A B C G 1(p=0.01)$. Furthermore, within the diabetes effect cohort there was a significant down regulation in the lipid biosynthesis gene SREBF1 $(p<0.001)$, however PPARY produced no change in gene expression when analysed with respect to diabetes effect $(p=0.26)$ (Table 4.12). 


\section{Plasma acyl-ghrelin levels in relation to lipid retention genes}

In contrast to the total sample cohort, plasma acyl-ghrelin levels and GHRL expression showed no association with plasma lipids within the diabetes effect cohort. Furthermore, neither demonstrated an association with key lipid retention genes (ABCG1; $\left.r_{\mathrm{s}}=-0.107, \mathrm{P}=0.770, \quad L X R B ; \mathrm{r}_{\mathrm{s}}=-0.150, \mathrm{P}=0.679\right)$. Finally, lipid biosynthesis gene, SREBF1 expression presented a significant association with GHRL expression $\left(r_{s}=0.803, p<0.01\right)$, this association was not seen with SREBF1 and plasma acyl-ghrelin levels $\left(r_{s}=0.011, p=0.977\right)$.

\begin{tabular}{|c|c|c|c|c|}
\hline Group & Gene of interest & Fold Change & Direction & P-value \\
\hline \multirow{3}{*}{$(\mathrm{NO}+\mathrm{O}) \vee$} & LXRB & $\mathbf{2 . 2 2}$ & $\downarrow$ & $<0.05$ \\
\cline { 2 - 5 } OT2D & ABCG1 & $\mathbf{2 . 2 2}$ & $\downarrow$ & $\mathbf{0 . 0 1}$ \\
\cline { 2 - 5 } & SREBF1 & $\mathbf{2 . 8 6}$ & $\downarrow$ & $<0.001$ \\
\cline { 2 - 5 } & PPARY & 0.77 & $\longleftrightarrow$ & 0.26 \\
\hline
\end{tabular}

Table 4.12. Relative fold change values for lipid retention gene expression data for diabetes effect cohort. P value deduced from $\Delta$ Ct T-Test. Significant values shown in bold $\downarrow=$ down regulation $\leftrightarrow$ = no overall change

\subsubsection{Inflammation markers}

\section{Plasma cytokine levels}

Analysis of cytokine data with respect to diabetes effect yielded no significant differences in pro-inflammatory markers IL-6 $(p=0.35)$ and TNF $\alpha(p=0.56)$. However, the anti-inflammatory marker IL-10 was significantly reduced within those with Type 2 diabetes in comparison with those without $(p<0.01)$ (Table 4.13). 


\begin{tabular}{|c|c|c|c|}
\hline $\begin{array}{c}\text { Cytokine } \\
{[\mathrm{pg} / \mathrm{mL}]}\end{array}$ & $\begin{array}{c}\text { No Diabetes } \\
(\mathrm{NO}+\mathrm{O}) \\
(\mathrm{n}=20)\end{array}$ & $\begin{array}{c}\text { Obese Type 2 } \\
\text { (OT2D) } \\
(\mathrm{n}=10)\end{array}$ & P-value \\
\hline IL-6 & 11.4 & 7.9 & 0.35 \\
\hline TNFa & 26.8 & 20.1 & 0.56 \\
\hline IL-10 & {$[10.9-42.1-15.1]$} & {$[14.0-32.6]$} & \\
\hline & 68.6 & 39.6 & $<0.01$ \\
\hline
\end{tabular}

Table 4.13. Cytokine levels for diabetes effect cohort. Median and interquartile range for cytokine levels for each group. P-value determined via independent T-Test comparing Diabetes (OT2D) vs No Diabetes (ND) cohorts. Significant values are shown in bold.

\section{$\underline{\text { Total Antioxidant Status }}$}

Analysis indicates a significant differences of average TAOS (\%) when the diabetes group was compared those with the no diabetes group ((NO + O) v OT2D; $48.8 v$ $31.1 \% ; p<0.001$ ) (Figure 4.7), showing decreased levels of antioxidant indicative of an increased levels of oxidative stress in the diabetes group.

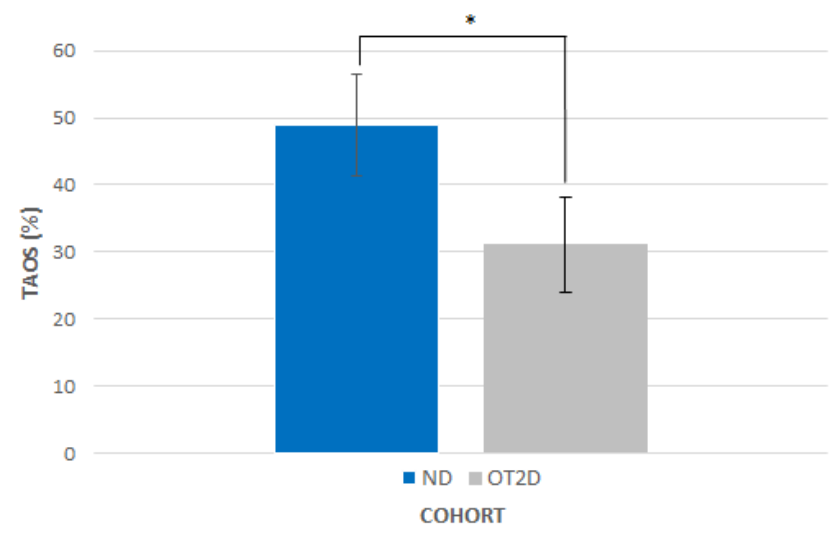

Figure 4.7. Bar graph of mean TAOS (\%) levels for diabetes effect. No diabetes $(N D(N O+O))(n=20)$ and diabetes (OT2D)(n=10). Statistical analysis was conducted using an independent T-Test. Statistical significance between groups are indicated by asterisks $\left({ }^{*} p<0.001\right)$. 


\section{Inflammation vs acyl-ghrelin}

During the assessment of inflammatory markers association with acyl-ghrelin and GHRL gene expression, it was evident that within the diabetes cohort all associations were diminished. Acyl-ghrelin showed no significant association with inflammatory cytokines (IL-6; $p=0.92$, TNF $\alpha ; p=0.29$ and IL10; $p=0.19$ ), nor with oxidative stress marker (TAOS\%; $\mathrm{p}=0.23$ ). In addition, GHRL expression had no associations with inflammatory or oxidative stress markers (IL-6 $(p=0.42)$, TNF $\alpha$ $(p=0.22)$, IL-10 $(p=0.09)$ or TAOS $\%(p=0.65))$ (Table 4.14).

\begin{tabular}{|l|l|l|l|}
\hline \multicolumn{2}{|l|}{} & $\begin{array}{l}\text { Plasma } \\
\text { acyl-ghrelin }\end{array}$ & $\begin{array}{l}G H R L \\
\Delta \mathrm{Ct}\end{array}$ \\
\hline IL-6 & $\begin{array}{l}\text { Spearman's Correlation } \\
\text { P-value }\end{array}$ & $\begin{array}{l}-0.042 \\
0.299\end{array}$ & -0.311 \\
& - & 0.415 \\
\hline TNF $\alpha$ & Spearman's Correlation & -0.600 & 0.667 \\
& P-value & 0.285 & 0.219 \\
\hline IL-10 & Spearman's Correlation & -0.700 & 0.821 \\
& P-value & 0.188 & 0.089 \\
\hline TAOS \% & Spearman's Correlation & -0.418 & -0.165 \\
& P-value & 0.229 & 0.649 \\
\hline
\end{tabular}

Table 4.14 Correlation data for gene expression, plasma acyl-ghrelin levels and inflammatory markers for diabetes effect cohort. Data analysis of relative $\Delta \mathrm{Ct}$ value and raw plasma marker levels $(n=10) . \Delta C t$ values are inverse on gene transcript level. Non-parametric data analysis via Spearman's rho correlation used to determine correlation between parameters. 


\subsubsection{Obesity effect}

The third arm of data analysis aimed to investigate the obesity effect (Non-Obese (NO) v Obesity (O+OT2D)). In order to determine whether obesity was causing an effect on associations between variables, analysis of correlations was conducted within samples in the obesity (O+OT2D) cohort only.

\subsubsection{Baseline characteristics}

The baseline characteristics within the obesity group $(\mathrm{O}+\mathrm{OT} 2 \mathrm{D})$ and the non-obese group (NO) demonstrated no significant difference within age $(p=0.45)$ or $\mathrm{HbA1c}$ $(p=0.74)$. However, as expected, there was a significant difference within the obese group for weight $(p<0.01), B M I(p<0.001)$ and fasting glucose levels $(p<0.05)$ (Table 4.15). 


\begin{tabular}{|c|c|c|c|}
\hline & $\begin{array}{c}\text { Obesity } \\
\text { (O+OT2D) } \\
(\mathrm{n}=20)\end{array}$ & $\begin{array}{c}\text { Non-Obese } \\
(\mathrm{NO}) \\
(\mathrm{n}=10)\end{array}$ & P-value \\
\hline Age (Years) & $48.3(9.9)$ & $51.8(15.5)$ & 0.45 \\
\hline Weight (Kg)* & $112.5[89-148]$ & $72.2[64-81]$ & $<0.01$ \\
\hline BMI (Kg/m²)* & $41.8[34-48]$ & $26.2[24-28]$ & $<0.001$ \\
\hline Glucose (mmol/L)* & $6.1[5.4-7.5]$ & $4.8[4.5-5.7]$ & $<0.05$ \\
\hline HbA1c (\%) & $5.5[5.3-7.0]$ & $5.0[4.6-5.7]$ & 0.74 \\
\hline HbA1c (mmol/mol)* & $36.6[33.6-52.7]$ & $30.6[26.3-39.1]$ & 0.07 \\
\hline Statin prescription ${ }^{*}$ & $30(6)$ & $20(2)$ & 0.56 \\
\hline (\%) & & & \\
\hline
\end{tabular}

Table 4.15. Baseline data for obesity effect cohort. Mean and standard deviation shown for normally distributed data \& $p$ val determined using one way ANOVA

* Median and interquartile ranges shown for data that is not normally distributed data and Kruskal Wallis used for p-value determination. "Categorical data tested using Pearson Chi-square analysis, \% shown with $\mathrm{n}$ in brackets. Significant values are shown in bold.

\subsubsection{Plasma levels of acyl-ghrelin}

Comparison of obesity and non-obese groups revealed no significant differences in circulating acyl-ghrelin levels (323.3 [101 - 480] v 466.0 [312 - 490] pg/mL; $p=0.48)$. Within the obesity group, fasting acyl-ghrelin was negatively correlated with BMI $\left(r_{s}=-0.58, p<0.01\right)$ and weight $\left(r_{s}=-0.55, p<0.05\right)$, respectively (Figure 4.8A-B). 


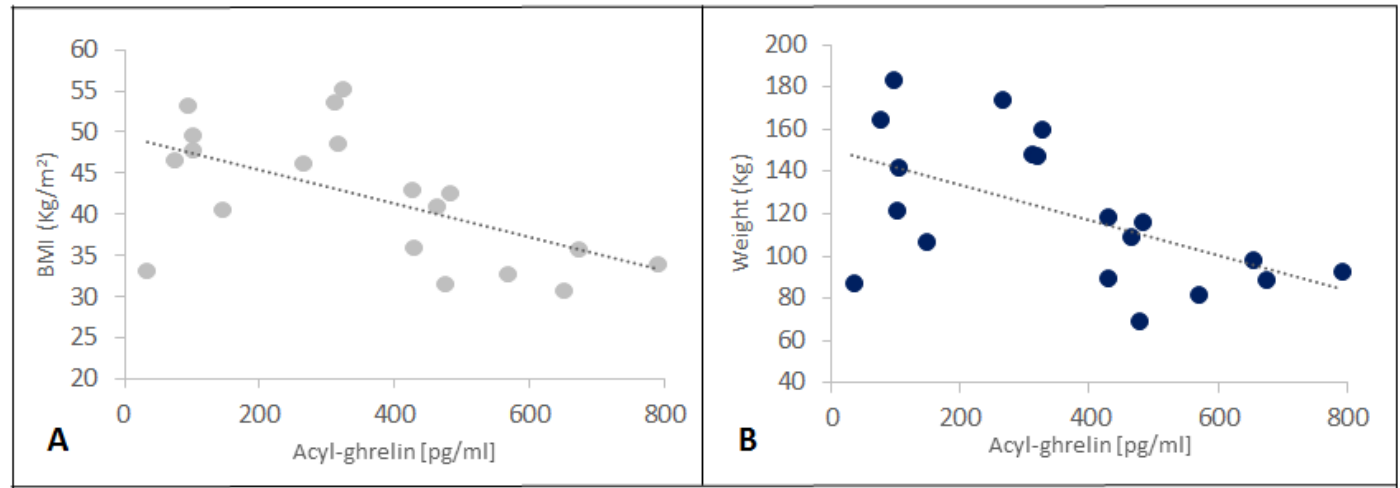

Figure 4.8A-B. Scatter graphs between acyl-ghrelin and BMI and acyl-ghrelin and body weight. ABMI $\left(r_{s}=-0.58, p<0.01\right), y=-0.0205 x+49.52, R^{2}=0.332(n=20)$. B- Body weight $\left(r_{s}=-0.55, p<0.05\right), y=-$ $0.0841 x+150.58, R^{2}=0.3044(n=20)$.

\section{Ghrelin gene axis expression across cohorts}

Gene expression data was analysed to signify whether obesity was influencing the expression of key ghrelin axis genes. Results confirm there was no significant differences in mBOAT4 $(\mathrm{p}=0.31)$ or LYPLA1 $(\mathrm{p}=0.44)$ expression between the two groups. Furthermore, in contrast to previous sections GHRL expression showed no fold change difference within the obese group versus the non-obese group $(p=0.47)$ (Table 4.16).

\begin{tabular}{|c|c|c|c|c|}
\hline Group & Gene of interest & Fold Change & Direction & P-Value \\
\hline \multirow{2}{*}{ NO v } & GHRL & 0.97 & $\longleftrightarrow$ & 0.47 \\
\cline { 2 - 5 } (O+OT2D) & mBOAT4 & 0.76 & $\longleftrightarrow$ & 0.31 \\
\cline { 2 - 5 } & LYPLA1 & 1.28 & $\longleftrightarrow$ & 0.44 \\
\hline
\end{tabular}

Table 4.16. Relative fold change values for ghrelin axis gene expression data for obesity effect cohort. P value deduced from $\Delta$ Ct T-Test.

$\downarrow=$ down regulation $\leftrightarrow$ = no overall change 


\section{Plasma acyl-ghrelin levels in relation to ghrelin gene axis}

Association of ghrelin axis gene expression and plasma acyl-ghrelin levels within the obese group indicated no significant correlation with either LYPLA1 $(p=0.571)$ or mBOAT4 $(\mathrm{p}=0.511)$, as with previous arms of the study. However, circulating acylghrelin levels were significantly association with GHRL $(p<0.05)$ (Table 4.17).

\begin{tabular}{|l|l|l|}
\hline \multicolumn{2}{|l|}{} & $\begin{array}{l}\text { Plasma } \\
\text { acyl- } \\
\text { ghrelin }\end{array}$ \\
\hline GHRL & Spearman's Correlation & $\mathbf{- 0 . 4 9 3}$ \\
& P-value & $\mathbf{0 . 0 3 2 *}$ \\
\hline \multirow{2}{*}{ YYPLA1 } & Spearman's Correlation & -0.139 \\
& P-value & 0.571 \\
\hline mBOAT4 & Spearman's Correlation & 0.161 \\
& P-value & 0.511 \\
\hline
\end{tabular}

Table 4.17 Correlation data for gene expression and plasma acyl-ghrelin for obesity effect cohort. Data analysis of relative $\Delta C t$ value and raw plasma marker levels $(n=20) . \Delta C t$ values are inverse on gene transcript level. Non-parametric data analysis via Spearman's rho correlation used to determine correlation between parameters. Significant values shown in bold.

\subsubsection{Lipid profiles}

Circulating lipid profiles in respect to the obese state, indicated no significant change across the two groups (cholesterol; $p=0.45, H D L ; p=0.43, L D L ; p=0.25$ and triglycerides; $p=0.14$ ) (Table 4.18). Upon analysis of acyl-ghrelin association with lipid profile markers, there was no significant association was present within the obese group (cholesterol; $r_{s}=0.291, p=0.23, H D L ; r_{s}=0.245, p=0.31, L D L ; r_{s}=0.281$, $\mathrm{p}=0.24$ and triglycerides; $\left.\mathrm{r}_{\mathrm{s}}=-0.462, \mathrm{p}=0.14\right)$. Due to $G H R L$ expression corresponding with acyl-ghrelin levels, it was also analysed with respect to lipid profile associations, which indicated a significant correlation between increased 
GHRL expression with increased plasma cholesterol and LDL levels $\left(r_{s}=-0.462\right.$, $p<0.05, r_{s}=-0.448, p<0.05$, respectively) (Figure 4.9A-B).

\section{Lipid retention gene expression across cohorts}

Contrary to other arms within this study, the obesity effect demonstrated no fold change alterations between key lipid retention genes when compared to the nonobese cohort (NO V (O + OT2D); LXRB $\mathrm{p}=0.10, A B C G 1=0.06$, SREBF1 $\mathrm{p}=0.14$ and PPARY $\mathrm{p}=0.18)$ (Table 4.19).

\section{Plasma acyl-ghrelin levels in relation to lipid retention genes}

Furthermore, no association was demonstrated between circulating acyl-ghrelin and key lipid retention genes ( $A B C G 1$ and $L X R B$ ), nor lipid biosynthesis genes (SREBF1). In addition, GHRL expression also deemed no significant relation with lipid retention genes ( $A B C G 1$ and $\angle X R B)$, however had a significant positive correlation with the lipid biosynthesis gene SREBF1 $\left(r_{s}=0.624, p<0.01\right)$.

\begin{tabular}{|c|c|c|c|}
\hline & $\begin{array}{c}\text { Obesity } \\
\text { (O+ OT2D) } \\
(\mathrm{n}=20)\end{array}$ & $\begin{array}{c}\text { Non-Obese } \\
(\mathrm{NO}) \\
(\mathrm{n}=10)\end{array}$ & P-value \\
\hline Cholesterol (mmol/L) & $3.9(1.5)$ & $4.4(1.2)$ & 0.45 \\
\hline $\mathrm{HDL}(\mathrm{mmol} / \mathrm{L})$ & $1.0(0.6)$ & $1.1(0.4)$ & 0.43 \\
\hline LDL (mmol/L) & $2.2(1.1)$ & $2.7(0.9)$ & 0.25 \\
\hline Triglycerides (mmol/L) & $1.7(0.7)$ & $1.2(1.0)$ & 0.14 \\
\hline
\end{tabular}

Table 4.18. Lipid profiles for obesity effect cohort. Mean and standard deviation shown for normally distributed data \& p-value determined using one way ANOVA. 


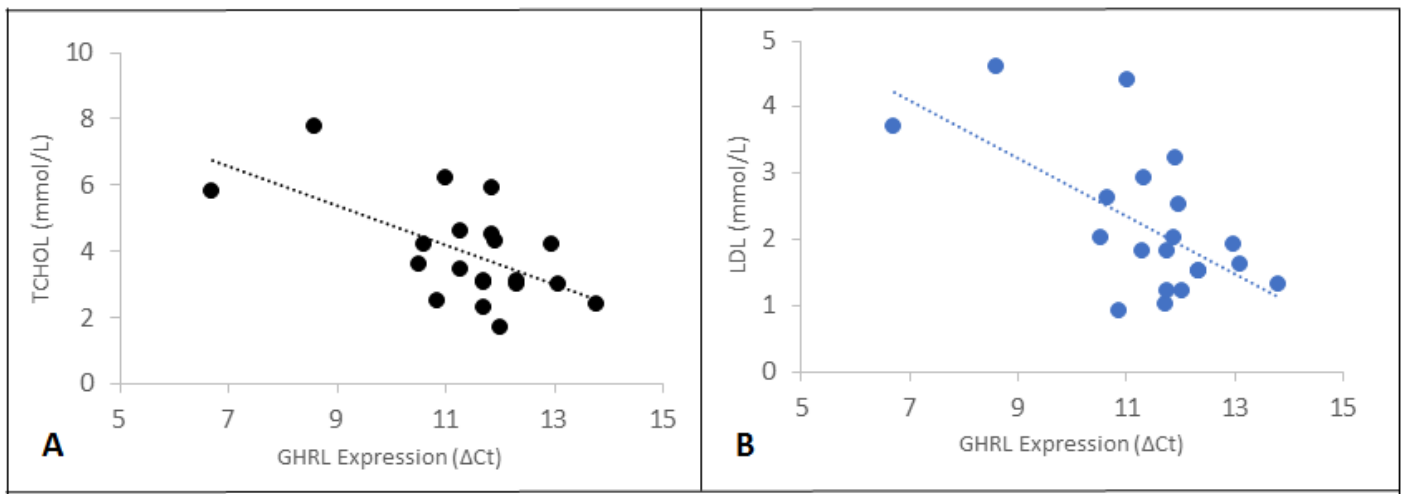

Figure 4.9A-B. Scatter graphs between GHRL expression and cholesterol, and GHRL expression and LDL. A- Plasma total cholesterol $\left(r_{s}=-0.462, p<0.05\right)(n=20) . y=-0.591 x+10.688, R^{2}=0.3589$. BPlasma low density lipoprotein $(L D L)\left(r_{s}=-0.448, p<0.05\right) y=-0.4337 x+7.1351, R^{2}=0.3863(n=20)$.

\begin{tabular}{|c|c|c|c|c|}
\hline Group & Gene of interest & Fold Change & Direction & P-value \\
\hline \multirow{2}{*}{ NO v } & LXR6 & 0.50 & $\longleftrightarrow$ & 0.10 \\
\cline { 2 - 5 } (O+OT2D) & ABCG1 & 0.63 & $\longleftrightarrow$ & 0.06 \\
\cline { 2 - 5 } & SREBF1 & 0.64 & $\longleftrightarrow$ & 0.14 \\
\cline { 2 - 5 } & PPARY & 0.69 & $\longleftrightarrow$ & 0.18 \\
\hline
\end{tabular}

Table 4.19. Relative fold change values for lipid retention gene expression data for obesity effect cohort. P-value deduced from $\Delta$ Ct T-Test.

$\downarrow=$ down regulation $\leftrightarrow=$ no overall change 


\subsubsection{Inflammation Markers}

\section{Plasma cytokine levels}

The obesity effect showed no significant differences in the level of proinflammatory markers IL-6 or TNF $\alpha$, however the anti-inflammatory marker IL-10 was significantly reduced within the obese group (NO v (O + OT2D); $55.6 \vee 40.8$, $p<0.01$ ) (Table 4.20).

\section{$\underline{\text { Total Antioxidant Status }}$}

Data analysis for obesity effect showed a significant difference in overall TAOS (\%) (NO v (O + OT2D); 53.5\% v 37.6\%; $p<0.001$ ) (Figure 4.10).

\begin{tabular}{|c|c|c|c|}
\hline $\begin{array}{c}\text { Cytokine } \\
{[\mathrm{pg} / \mathrm{mL}]}\end{array}$ & $\begin{array}{c}\text { Obesity } \\
(\mathrm{O}+\text { OT2D }) \\
(\mathrm{n}=20)\end{array}$ & $\begin{array}{c}\text { Non-obese } \\
(\mathrm{NO}) \\
(\mathrm{n}=10)\end{array}$ & P-value \\
\hline IL-6 & 10.7 & 12.6 & 0.73 \\
& {$[7.7-18.6]$} & {$[7.0-20.4]$} & 0.31 \\
\hline TNF $\alpha$ & {$[16.0-39.5]$} & {$[5.1-43.4]$} & \\
\hline IL-10 & 40.8 & 55.6 & $<0.01$ \\
& {$[26.9-59.6]$} & {$[66.3-129.5]$} & \\
\hline
\end{tabular}

Table 4.20. Cytokine levels for obesity effect cohort. Median and interquartile range for cytokine levels for each group. P-value determined via independent T-Test comparing obesity effect. Significant values shown in bold. 


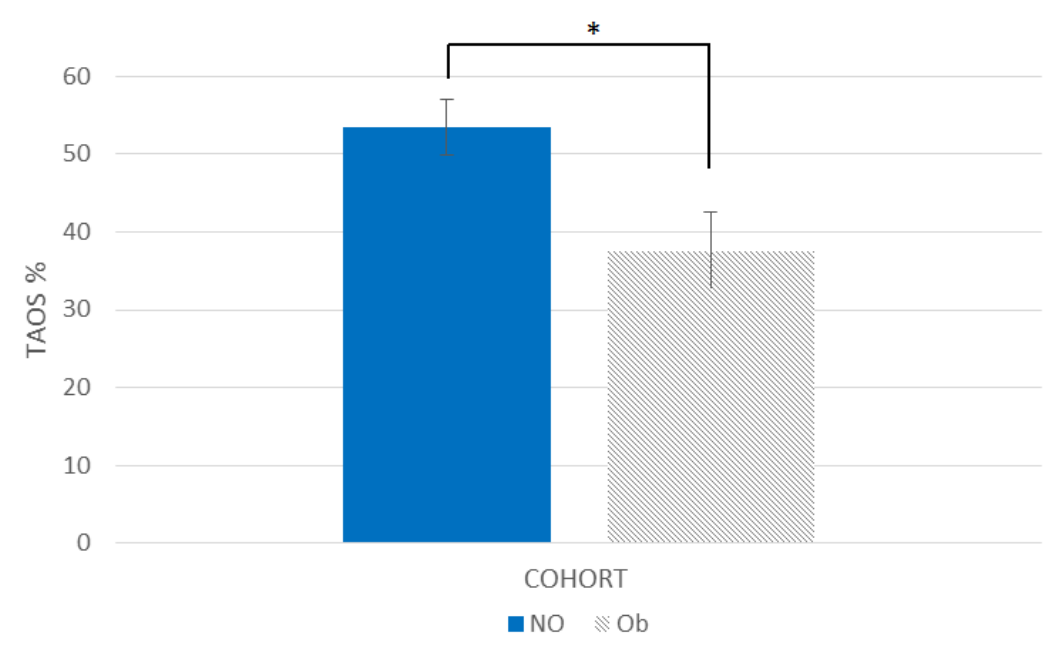

Figure 4.10. Bar graph of mean TAOS (\%) shown for obesity effect. Non-obese (NO)(n=10) and obesity effect $(O b(O+O T 2 D))(n=20)$. Statistical analysis was conducted using an independent TTest. Statistical significance between groups are indicated by asterisk ( $\left.{ }^{*} p<0.001\right)$.

\section{Inflammation vs acyl-ghrelin}

Plasma acyl-ghrelin showed no significant association with inflammatory cytokines within the obese group (IL-6, $\mathrm{p}=0.59 ; \mathrm{TNF} \alpha, \mathrm{p}=0.47 ; \mathrm{IL}-10, \mathrm{p}=0.63$ ). Inflammatory cytokines also indicated no association with GHRL expression with respect to IL-6 and TNF $\alpha$. However, pro-inflammatory marker IL-10 demonstrated a significant association with $\triangle \mathrm{Ct}$ values of GHRL expression. TAOS (\%) indicated oxidative stress was also independent of plasma acyl-ghrelin levels $(p=0.32)$, however when TAOS (\%) is analysed versus ghrelin gene expression it was indicative that an increase in GHRL expression was associated with an increase in TAOS (\%) (GHRL $\triangle \mathrm{Ct}$ vs TAOS \%; p<0.05) (Table 4.21). 


\begin{tabular}{|l|l|l|l|}
\hline \multicolumn{2}{|l|}{} & $\begin{array}{l}\text { Plasma } \\
\text { acyl-ghrelin }\end{array}$ & $\begin{array}{l}G H R L \\
\Delta \mathrm{Ct}\end{array}$ \\
\hline IL-6 & Spearman's Correlation & -0.136 & -0.025 \\
& P-value & 0.590 & 0.918 \\
\hline TNF $\alpha$ & Spearman's Correlation & -0.261 & 0.419 \\
& P-value & 0.467 & 0.199 \\
\hline IL-10 & Spearman's Correlation & 0.176 & $\mathbf{- 0 . 5 7 3}$ \\
& P-value & 0.627 & $\mathbf{0 . 0 4 6}$ \\
\hline TAOS \% & Spearman's Correlation & 0.242 & $\mathbf{- 0 . 5 3 6}$ \\
& P-value & 0.318 & $\mathbf{0 . 0 1 5 *}$ \\
\hline
\end{tabular}

Table 4.21 Correlation data for gene expression, plasma acyl-ghrelin and inflammatory markers for obesity effect cohort. Data analysis of relative $\Delta C$ t value and raw plasma marker levels $(n=20) . \Delta C t$ values are inverse on gene transcript level. Non-parametric data analysis via Spearman's rho correlation used to determine correlation between parameters. Significant values shown in bold. 


\subsection{Discussion}

\subsubsection{Circulating acyl-ghrelin levels}

Gene expression data of the ghrelin axis provided an insight into the regulation of circulating ghrelin within obese individuals with and without T2D. Data suggested that a down regulation of GHRL was present within individuals with T2D, however this effect was not mirrored in obese individuals. Previously published literature confirms this study's findings by indicating that low total ghrelin levels are significantly associated with insulin resistance (Pöykkö et al., 2003, Shiiya et al., 2002). However, this was initially deemed to be due do a decline in desacyl-ghrelin, with acyl-ghrelin being increased within obese individuals with T2D (Rodríguez et al., 2012). However, it is evident across the literature that acyl-ghrelin levels are disputed, with other reports indicating a significant decline in acyl-ghrelin levels within obese individuals with T2D (Dardzińska et al., 2014) and those with gestational diabetes (Supák et al., 2016). Within this study, there is also paradoxical evidence with data suggesting no change in acyl-ghrelin concentrations in an obese state when compared to non-obese, yet a marked decline in acyl-ghrelin levels within obese individuals with T2D. Investigation of the ghrelin axis through gene expression has indicated that the expression of key regulatory enzymes in pre-proghrelin modification are unaltered throughout the total and disease effect cohorts, suggesting that acyl-ghrelin plasma concentrations may be correlated specifically with GHRL expression. Acyl-ghrelin levels within T2D are therefore hypothesised to be under the regulation of GHRL transcription itself, rather than an alteration in the mechanism involved in regulation of the octanoylation of ghrelin. Furthermore, this corresponds with a decline in GHRL expression, producing a lower plasma desacyl- 
ghrelin level as often reported within the metabolic state (Rodríguez et al., 2012). Analysis of desacyl-ghrelin within participants would also have been advantageous within the sample cohorts to establish whether acyl-ghrelin and desacyl-ghrelin share a similar or opposing relationship within the study, however due to timing constraints this was not possible.

A decline in acyl-ghrelin levels and GHRL gene expression were associated with a rise in fasting plasma glucose levels across the total sample cohort indicating that the down regulation of ghrelin could be dependent on glucose homeostasis. This corresponds with previously published data, which suggests an inverse correlation with acyl-ghrelin levels and homeostatic model assessment of insulin resistance (HOMA-IR) (Qarni et al., 2017). Therefore, a reduction of acyl-ghrelin levels in long term hyperglycaemia may not be due to post-translational modifications, but rather a disruption in the transcription of the GHRL gene itself. These findings correlate with a study undertaken in human individuals with T1D, T2D and normoglycaemia which indicated ghrelin secretion was suppressed by long term poor glycaemic control (Hiroaki et al., 2007). However, acyl-ghrelin's association with plasma glucose was lost when data were analysed with respect to diseases effect cohorts. This could be due to data within the disease effect cohorts consisting of half the participants, which resulted in a loss in statistical power and an increase in variation (Browner et al., 2007). Additional exploratory analysis into disease state does however allow for the development of associations within the data that could be further explored in additional studies to provide greater statistical power and to eradicate type 1 statistical errors. 


\subsubsection{Acyl-ghrelin mediated lipid retention}

Within this chapter, the relationship of acyl-ghrelin and lipid retention was further examined in relation to adipose tissue as a complex source of various tissue types, rather than acyl-ghrelin's effect directly on mature adipocytes as discussed in Chapter 3. Analysis of the findings indicate contrasting data that shows that within non-obese individuals, higher levels of circulating acyl-ghrelin coincides with an increase in key lipid transport gene expression. This could be due to acyl-ghrelin's effect on adipose tissue as a whole, including macrophages, which have shown to induce an increase in lipid retention gene mRNA levels in the presence of acylghrelin treatments (Demers et al., 2009). Even though acyl-ghrelin may be involved in mediating lipid retention, this effect however is diminished on a global scale and is only apparent within a local system. Previously published work demonstrates that within mice, the effect of acyl-ghrelin is the same, both locally in adipocytes (3T3L1) (Miegueu et al., 2011) and globally (WAT depot) (Churm et al., 2017, Porteiro et al., 2013)(Table 4.22).

In addition to a local versus global effect of acyl-ghrelin, a further explanation that may account for the difference is the degree and duration of a hyperglycaemic state. Previously reported evidence shows that hyperglycaemia has demonstrated a regulatory role in the expression of key lipid retention genes including ABCG1 (Mauerer et al., 2009) and LXR (Mitro et al., 2007). 


\begin{tabular}{|c|c|c|c|c|}
\hline Model & Dose & Treatment & Effect & Reference \\
\hline $\begin{array}{l}\text { 3T3-L1 } \\
\text { (Murine } \\
\text { adipocytes) }\end{array}$ & $\begin{array}{l}\text { Acyl-ghrelin } \\
\text { 1-100 pmol, } \\
\text { Desacyl- } \\
\text { ghrelin 1- } \\
100 \text { pmol }\end{array}$ & $\begin{array}{l}\text { Preadipocytes } \\
\text { incubated with } \\
\text { desacyl-ghrelin and } \\
\text { acyl-ghrelin for } \\
24 \text { hours }\end{array}$ & $\begin{array}{l}\text { Desacyl and acyl- } \\
\text { ghrelin- } \\
\text { stimulated } \\
\text { adipogenesis, } \\
\text { fatty acid uptake } \\
\text { and inhibited } \\
\text { lipolysis }\end{array}$ & $\begin{array}{l}\text { (Miegueu } \\
\text { et al., } \\
\text { 2011) }\end{array}$ \\
\hline $\begin{array}{l}\text { Perigonadal } \\
\text { adipose tissue } \\
\text { (Murine) }\end{array}$ & $\begin{array}{l}\text { Acyl-ghrelin } \\
30 \mu g \text { day }\end{array}$ & Daily ip injection & $\begin{array}{l}\text { mRNA levels of } \\
\text { several } \\
\text { adipogenic and } \\
\text { fat-storage- } \\
\text { promoting } \\
\text { enzymes were } \\
\text { up-regulated }\end{array}$ & $\begin{array}{l}\text { (Porteiro } \\
\text { et al., } \\
\text { 2013) }\end{array}$ \\
\hline $\begin{array}{l}\text { THP-1 } \\
\text { (Human } \\
\text { macrophages) }\end{array}$ & $\begin{array}{l}\text { 1, } 10 \& 100 \\
\text { nM Acyl- } \\
\text { ghrelin }\end{array}$ & $\begin{array}{l}\text { Treated PMA- } \\
\text { differentiated THP- } \\
1 \text { macrophages } \\
\text { with increasing } \\
\text { doses of ghrelin }\end{array}$ & $\begin{array}{l}\text { Increases in } \\
\text { mRNA levels of } \\
\text { LXR and ABC } \\
\text { isoforms }\end{array}$ & $\begin{array}{l}\text { (Demers } \\
\text { et al., } \\
\text { 2009) }\end{array}$ \\
\hline
\end{tabular}

Table 4.22. Effects of acyl-ghrelin treatment on adipogenesis in both local and global adipose regions, table taken in part from (Churm et al., 2017). 


\subsubsection{Acyl-ghrelin and the inflammatory state}

Relevant findings within this chapter indicate no overall change in plasma levels of pro-inflammatory markers across groups, indicating no correlation to acyl-ghrelin levels or GHRL expression. This is counter to the published IL-6 literature which indicates that the enhancement of the metabolic state causes an increase in proinflammatory markers such as IL-6 (Maachi et al., 2004, Bastard et al., 2006, Bastard et al., 2002, Makki et al., 2013). In addition, TNFa was initially reported 20 years ago to be increased within obesity and T2D (Saghizadeh et al., 1996, Löfgren et al., 2000, Bastard et al., 2006, McLaughlin et al., 2017), however findings differ amongst publications with studies often reporting no significant alterations in TNF $\alpha$ levels between both disease states and a control cohort (Frittitta et al., 1997, Koistinen et al., 2000, Borst, 2004). A lack of association seen within the proinflammatory marker levels during the progression of the metabolic state may not be apparent due to variation within samples and the relatively small sample size assessed here. Another plausible explanation is the role of prescribed medication within the sample cohorts. For instance, meta-analysis data suggests a role for metformin in the amelioration of chronic inflammation via a reduction of key inflammatory markers such as C-reactive protein, IL-6 and TNF $\alpha$ in vivo in humans (Wang et al., 2017) and in vitro in rodents (Jing et al., 2017).

Upon exploration of disease effect, it is apparent that both obesity and Type 2 diabetes caused a marked decline in anti-inflammatory and an increase in oxidative stress i.e. plasma IL-10 (van Exel et al., 2002, Esposito et al., 2003) and TAOS levels (Fenkci et al., 2003, Prior et al., 2017). Furthermore, within the total sample cohort and those within the obese effect cohort, there was a significant 
association between GHRL expression and IL-10. However, this association was diminished within the diabetes effect cohort. An improvement in IL-10 and TAOS (\%) data are both associated with the up regulation of GHRL expression, corresponding with published studies that indicate a promotion of inflammatory health in the presence of ghrelin (Suematsu et al., 2005, Harvey et al., 2017). Even though GHRL expression is significantly linked to acyl-ghrelin circulation, whether this inflammatory protection is due to an increase in total ghrelin, desacyl-ghrelin or acyl-ghrelin is not determined. Acyl-ghrelin has been linked to a plausible role in protecting human lens epithelial cells (Bai et al., 2017) and osteoblastic cells (Dieci et al., 2014) against ROS accumulation. Furthermore, as discussed in Chapter 3, upon acyl-ghrelin dosage of human mature adipocytes there is a promotion in a protective inflammatory response and an increase in anti-inflammatory markers. In addition to acyl-ghrelin, desacyl-ghrelin treatment has also established a protective role from oxidative stress in microvascular endothelial cells via regulation of SIRT1 catalytic activity (Shimada et al., 2014) and within osteoblastic cells independent of GHSR1 $\alpha$ (Dieci et al., 2014).

\subsection{Conclusion}

Within the data sets, it is evident that acyl-ghrelin associations are only present within the total sample cohort, indicating values within the diseased state are too extreme or sample size to small too assess and reveal any relationships. mRNA levels of GHRL are indicative of a positive association with an increase in plasma lipid markers across all study arms. Within both a diabetes and obesity effects there is an association of SREBF1 mRNA levels, suggesting the transcription of GHRL gene 
is associated with an increase in lipid biosynthesis and in turn an increase in circulating lipid profiles. Previous literature and results from this chapter conclude that total ghrelin; a combination of both acyl- and desacyl-ghrelin, is associated with a protective response to oxidative burden.

\subsection{Limitations}

Within this section, TAOS was measured as a marker of antioxidant status. However, in addition to this surrogate marker, it would have been beneficial to encompass additional analysis including thiobarbituric acid reactive substances (TBARS) assay (a measure of lipid peroxidation) and telomere length (a measure of DNA damage due to cellular oxidative burden).

The main limitation within this chapter is the analysis of sub-section data. The use of ex vivo samples allowed for the exploration of acyl-ghrelin mediated response, however, the analysis of association must be taken with caution. Due to the statistical approach used to elucidate disease effect, large amounts of comparisons and association analyses within the sub-sections were conducted, therefore there is a potential of Type 1 errors and the analysis of a smaller sample number. Increasing sample size would allow for the representation of this statistical analysis with greater power, however, time restraints did not allow for this to occur. In addition to this, upon analysis it is apparent that within the $\mathrm{O}$ and OT2D groups, there was a significant difference in weight and BMI. Upon further consideration, analysis could have benefited from a stricter inclusion criteria specifying a range within weight and $\mathrm{BMI}$ to ensure continuity within the weight between the two groups. 
Chapter 5

The effect of combining metformin and acylghrelin exposure on lipid retention and inflammation 


\subsection{Introduction}

\subsubsection{Type 2 diabetes medication}

Early stage T2D can often be managed through an improvement in diet and/or exercise. However, as the disease progresses, many individuals also require the use of hyperglycaemia medication or insulin therapy. Evaluating pharmacological agents as treatment for individuals who have $\mathrm{T} 2 \mathrm{D}$ and are obese or overweight (OT2D), requires considerations due to the effects that some hyperglycaemic agents may have on weight management. Hyperglycaemic therapy can be split into three distinct pharmaceutical groupings:- (i) treatments associated with weight gain- insulin secretagogues, thiazolidinediones and insulin, (ii) treatments that are weight neutral- metformin and dipeptidyl peptidase 4 (DPP-4) inhibitors, (iii) treatments linked to weight loss including-amylin mimetics and GLP-1 agonists and Sodium-glucose co-transporter-2 inhibitors (SGLT2i) (American Diabetes Association, 2017). Most pharmaceutical agents work in a manner to either promote insulin secretion or increase insulin sensitivity.

\subsubsection{Treatment guidelines}

The National Institute for Health and Care Excellence (NICE) guidelines for the management of T2D in adults directs the recommended treatment course based on up to date evidence and key developments. The algorithm for blood glucose lowering therapy in adults with T2D states initial drug therapy is dependent on HbA1c rise when following lifestyle intervention, with the introduction of metformin as a first line therapy (National Institute Health and Care Excellence, 2015). Metformin treatment can either be as monotherapy or in addition to other 
oral medications as dual or triple therapy (National Institute Health and Care Excellence, 2015). Alternatively, insulin therapy is recommended if (i) an individual is symptomatically hyperglycaemic, (ii) the dual or triple therapy is ineffective, or (iii) contraindications are present to metformin treatment and $\mathrm{HbA} 1 \mathrm{c}$ raises above $58 \mathrm{mmol} / \mathrm{mol}$ (7.5\%) (National Institute Health and Care Excellence, 2015).

\subsubsection{Metformin}

Metformin (1,1-dimethylbiguanide hydrochloride) is the most commonly prescribed oral medication worldwide that acts to lower blood glucose (Bailey, 2017, Griffin et al., 2017). The therapy is often used as a foundation therapy for individuals with newly diagnosed T2D due to a reputation of effective glucose lowering abilities, low cost, and good safety profile (Sanchez-Rangel and Inzucchi, 2017, Inzucchi et al., 2015).

Reflective of metformin being a first line diabetes therapy that is weight neutral, data analysis of individuals recruited within Chapter 4 showed $90 \%(n=9)$ of the OT2D group were receiving routine metformin treatment with the additional $10 \%(n=1)$ being undisclosed.

First noted for its lipid lowering abilities post-extraction from the plant Galega officinalis or French lilac in the 1920s, it was not until the 1950s that metformin was introduced as a treatment of adult onset diabetes in the USA, after studies by Jean Sterne in 1957 (Sterne, 1957), then in the UK and other European countries in 1958 (Bailey, 2017). Despite metformin's popularity as a first-line therapy, there is still debate as to the exact mechanism of action. Evidence suggests a reduction in hepatic glucose production being predominant, but there is 
fundamental evidence that metformin is also involved in the regulation of incretin hormones and can lower insulin resistance (Sanchez-Rangel and Inzucchi, 2017, Rena et al., 2017).

\subsubsection{Proposed mechanism of metformin action}

Metformin primarily acts to inhibit hepatic gluconeogenesis and increase insulin action (or sensitivity) in other organs i.e. fat (Moreno-Navarrete et al., 2011). Metformin is known to have a regulatory effect on key lipid metabolic pathways. For example metformin administration in rodent cells and in vivo results in the activation of AMPK, which supresses the expression of the $L X R$ ligands and SREBF-1 (Yang et al., 2009, Yap et al., 2011, Hayashi et al., 2014). In addition to regulating key lipogenic genes, metformin also demonstrates a relationship with the ghrelin axis via AMPK. Gagnon and colleagues reported that administration of metformin in rats was associated with lower circulating levels of acyl-ghrelin via diminished protein secretion and reduced mRNA transcription (Gagnon et al., 2013). Furthermore, both effects were blocked when treated with an AMPK inhibitor compound (Gagnon et al., 2013). Independent studies report the intrinsic effect of metformin treatment on acyl-ghrelin or lipogenic factors, however further exploration is required to understand whether acyl-ghrelin could alter metformin's action, or vice versa, on lipid metabolism and resultant inflammation within humans. In addition to the molecular mechanism of metformin, there is also a notably defined physiological route. Physiologically it is evident that metformin acts both directly and indirectly on the gut to increase glucose utilisation, increase GLP-1 levels and alter gut microbiome (Rena et al., 2017, McCreight et al., 2016) all of 
which highlight the vast network of pathways in which metformin plays a key role. Furthermore, there are variations in independent studies as the effect is altered dependent on cell or tissue type, dosage application and animal model used (Rena et al., 2017). Review of published data allows for a summary of the role of metformin on various site specific action, however very little published data indicates an effect of metformin on adipose tissue within humans (Bailey, 2017, Griffin et al., 2017, Rena et al., 2017, McCreight et al., 2016).

Due to metformin having a pre-defined intrinsic effect on the lipogenesis pathway, the interaction between hyperglycaemia, metformin exposure and acylghrelin needs elucidation within human mature adipocytes. 


\subsection{Aim}

As metformin is the first line treatment and most commonly prescribed treatment of T2D, it is vital to understand its interaction with acyl-ghrelin and the resulting effect on lipid retention and inflammation. I predict that the evaluation of metformin role would elucidate a common pathway between itself and acylghrelin's action. Thus ameliorating acyl-ghrelin's effect on lipid retention genes and further promoting inflammatory health. This chapter aimed to:-

1. Explore the effect of metformin on lipid retention genes in mature adipocytes in a hyperglycaemic environment

2. Explore the effect of metformin on inflammatory markers IL-6 and IL-10, in mature adipocytes in a hyperglycaemic environment

3. Investigate the combined effect of acyl-ghrelin and metformin on lipid retention and inflammation 


\subsection{Methods}

\subsubsection{SGBS cell treatment}

Human pre-adipocytes (SGBS cells) were revived, cultured, seeded into six well plates and differentiated as previously described in Section 2.1.2. Mature adipocytes were pre-exposed to a hyperglycaemic environment [ $25 \mathrm{mM}$ glucose] at day 12. The cells were then dosed with metformin [5nM] and/or acyl-ghrelin [100 $\mathrm{nM}$ ] or PBS vehicle to act as a control for all treatments on day 14 . For dual exposure, cells were pre-exposed to acyl-ghrelin, 30 minutes prior to the addition of metformin. Due to the novelty of this work, cell treatment dose and timings were based on alternative human cell lines or primary adipocyte cultures, as previously described in 3T3-L1 (Barbato et al., 2013) and Poietics ${ }^{\text {TM }}$ human visceral adipocytes (Fujita et al., 2016). Metformin (Sigma-Aldrich) was dissolved in PBS and added in serum-free culture medium at a final concentration of $5 \mathrm{nM}$. Cell experiments were conducted over 17 hours following the addition of metformin, as previous work (Chapter 3) indicated this was a sufficient incubation period to report cellular changes resulting from the addition of acyl-ghrelin.

\subsubsection{Oil Red O staining}

After 17 hours of exposure to metformin, and/or acyl-ghrelin or a PBS vehicle, cells underwent Oil red $\mathrm{O}$ analysis as described in Section 2.1.6, based on Mehlem and colleagues published protocol (Mehlem et al., 2013). All images were analysed using ImageJ software (Version 2) using the same procedure as discussed in Section

\subsection{3.}




\subsubsection{Gene expression analysis}

To explore whether metformin altered acyl-ghrelin mediated lipid retention, cell treatments underwent relative gene expression analysis. Gene expression data was analysed within those treated with metformin and/or acyl-ghrelin or vehicle. Analysis was conducted on key lipid retention genes; $L X R B, A B C G 1$ and SREBF1. RNA was extracted from cells (Section 2.1.8) 17 hours post-exposure, then underwent Real Time PCR (Section 2.3), and raw Ct values analysed using the $2^{-\Delta \Delta C T}$ method (Section 2.3.4) (Appendix 7).

\subsubsection{Adipokine analysis}

As demonstrated in earlier chapters, acyl-ghrelin has a pronounced effect on IL-6 and IL-10. These were analysed within cell supernatant extracted 17 hours posttreatment exposure via commercially available ELISA (Quantikine ${ }^{\circledR}$, R\&D systems) as described within the manufacturers' guidelines and Section 2.4. 


\section{$5.4 \quad$ Results}

\subsubsection{Effect of metformin on human mature adipocytes}

\subsubsection{Lipid area}

Intracellular lipid area was recorded and analysed to elucidate metformin's effect on mature adipocytes in terms of lipid area versus a control. Overall lipid area was unaltered with metformin treatment compared to control (Met v Veh; 3870.3 [3177 - 4463] $\mu \mathrm{m}^{2} v 3592.5[2491-4696] \mu \mathrm{m}^{2} ; p=0.35$ ) (Figure 5.1).

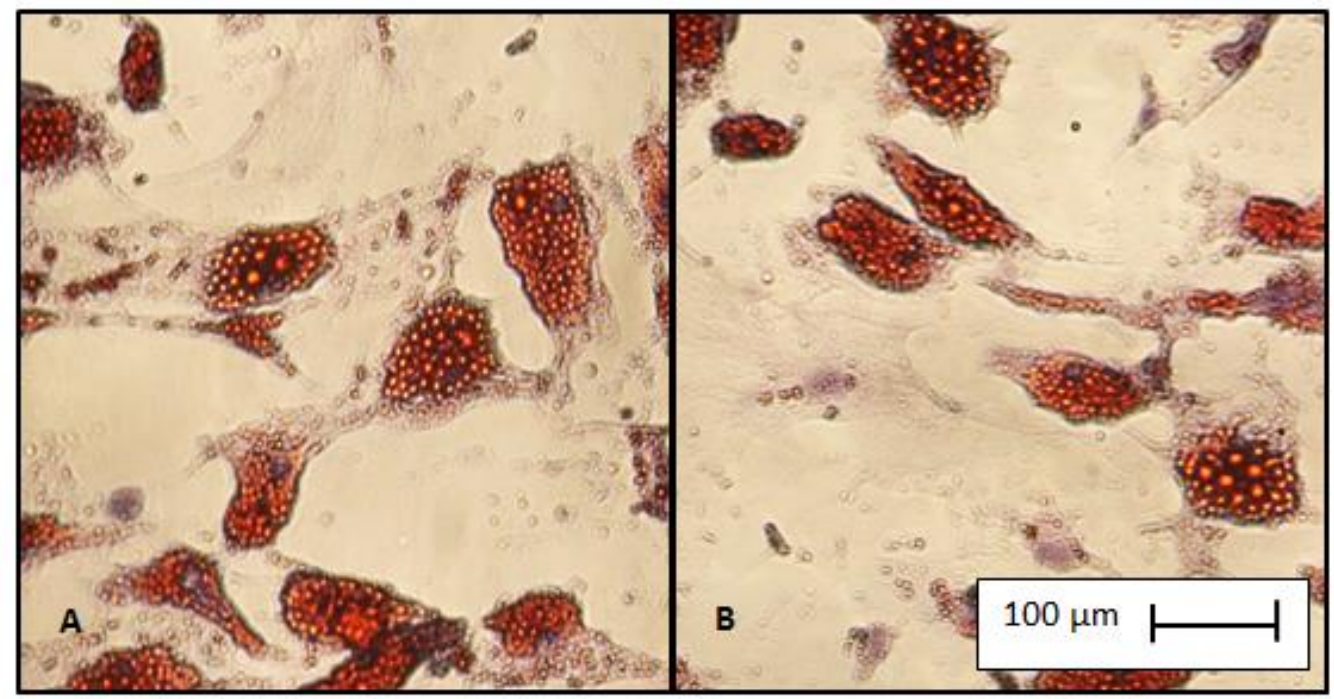

Figure 5.1. Oil red $O$ imaging of mature SGBS cells in $25 \mathrm{mM}$ (hyperglycaemia) glucose and metformin treatment at x50 magnification. Red stain- lipid droplets; purple stain- nuclei. (A) Cells grown in presence of metformin for 17 hours. (B) Cells grown in presence of PBS control for 17 hours.

\subsubsection{Real Time gene expression}

Analysis of lipid retention genes showed that after 17 hours of metformin exposure, mature adipocytes, compared to control, had a significant up regulation of $L X R B$, $A B C G 1$ and SREBF1. In order of fold change magnitude, results showed an up 
regulation of; $3.5( \pm 0.1)$ fold change for $L X R B(p<0.001), 33.1( \pm 1.9)$ fold change for ABCG1 $(p<0.001)$ and $3.4( \pm 0.8)$ fold change for SREBF1 $(p<0.001)$ (Figure 5.2).

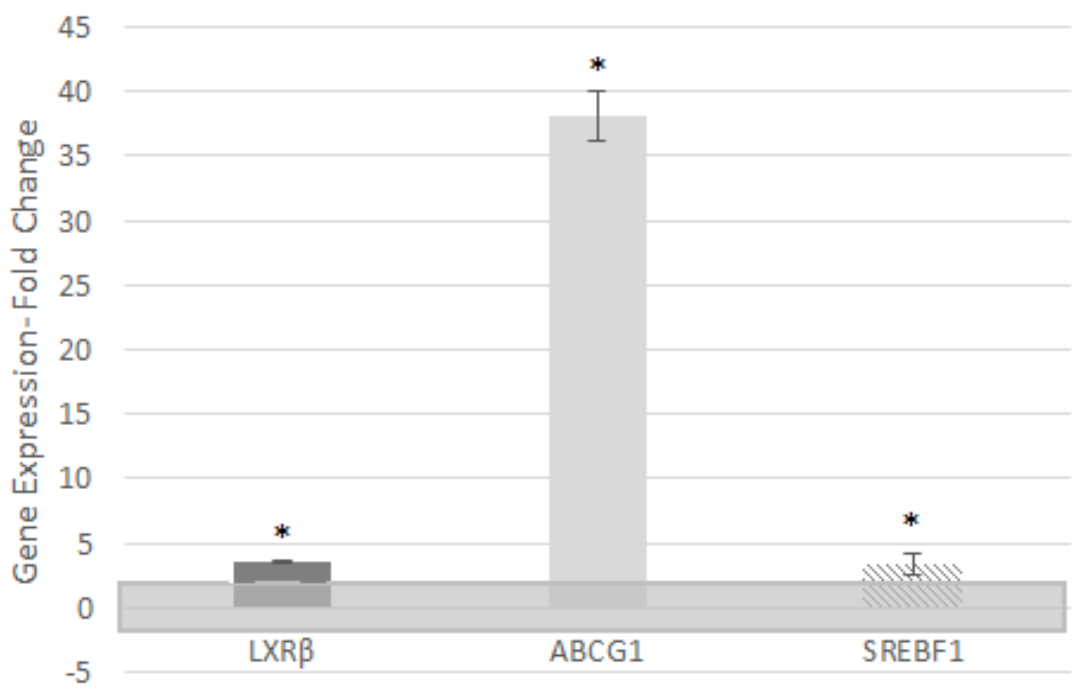

Figure 5.2. Relative fold change values for gene expression data in metformin treated cells in a hyperglycaemic environment [25 mM] in comparison to control at 17 hours. Fold change between 1.5 and 1.5 is classed as no relative gene expression change (grey region). Error bars indicate standard deviation between $\Delta \Delta C$ t of experimental repeats $(n=6) .{ }^{*}$ indicates a significant $(p<0.001)$ difference of $\Delta \mathrm{Ct}$ in metformin treated cells versus control treated cells, determined using an independent t-test.

\subsubsection{Adipokine levels}

Metformin exposure resulted in no significant alteration in IL-10 levels compared to a control (Met v Veh; 282.5 [252.0 - 299.5] pg/mL v 258.2 [244.6 - 282.9] pg/mL; $p=0.44$ ) (Figure 5.3A). However, the amount of IL-6 present was significantly lower following metformin exposure (Met v Veh; $23.2[19.3-25.6] \mathrm{pg} / \mathrm{mL}$ v 89.5 [87.7 95.5] pg/mL; $p<0.01$ ) (Figure 5.3B). 


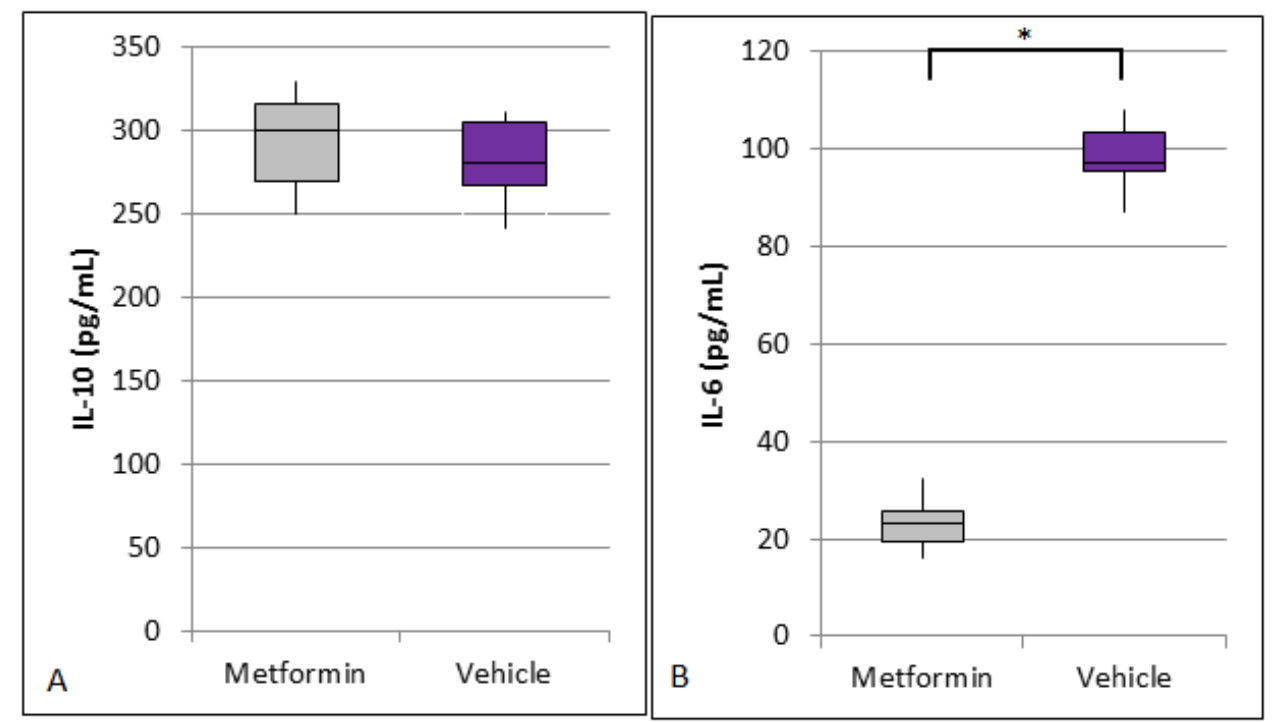

Figure 5.3. Box plot of IL-10 and IL-6 levels within metformin and control cells in a hyperglycaemic environment. (A) $\mathrm{IL}-10[\mathrm{pg} / \mathrm{mL}]$. (B) $\mathrm{IL}-6[\mathrm{pg} / \mathrm{mL}]$. Statistical analysis was conducted using non parametric measures ( $n=6$ per treatment). The Kruskal Wallis test determined statistical significance $(p<0.01)$ between groups which are indicated by *

\subsubsection{Effect of metformin in combination with acyl-ghrelin exposure on human} mature adipocytes

Within this results section, acyl-ghrelin only treatment data has been utilised from Chapter 3 to allow for the comparison of the effect of metformin in combination or as a monotherapy on SGBS cells in relation to acyl-ghrelin mediated lipid retention.

\subsubsection{Lipid area}

\section{Comparison to vehicle}

The combination of acyl-ghrelin with metformin significantly increased intracellular lipid area when compared to a vehicle control (Figure 5.4) (Veh v Met + AG; 3592.5 $\left.[2491-4696] \mu m^{2} v 4539.5[3773-5501] \mu m^{2} ; p<0.001\right)$. 


\section{Comparison to metformin}

The presence of acyl-ghrelin in combination with metformin caused significant increase in cellular lipid area when compared to metformin-only treated cells (Figure 5.4) (Met + AG v Met; $4539.5[3773-5501] \mu \mathrm{m}^{2} v 3870.3[3177-4463] \mu \mathrm{m}^{2}$; $\mathrm{p}<0.001)$.

\section{Comparison to acyl-ghrelin}

There was a significant decrease in the cellular lipid area of those cells exposed to both metformin and acyl-ghrelin in comparison to those treated with only acylghrelin (Met + AG v AG; $4539.5[3773-5501] \mu \mathrm{m}^{2} \vee 4788.7[4040-5976] \mu \mathrm{m}^{2}$; $p<0.05$ ) (Figure 5.4).

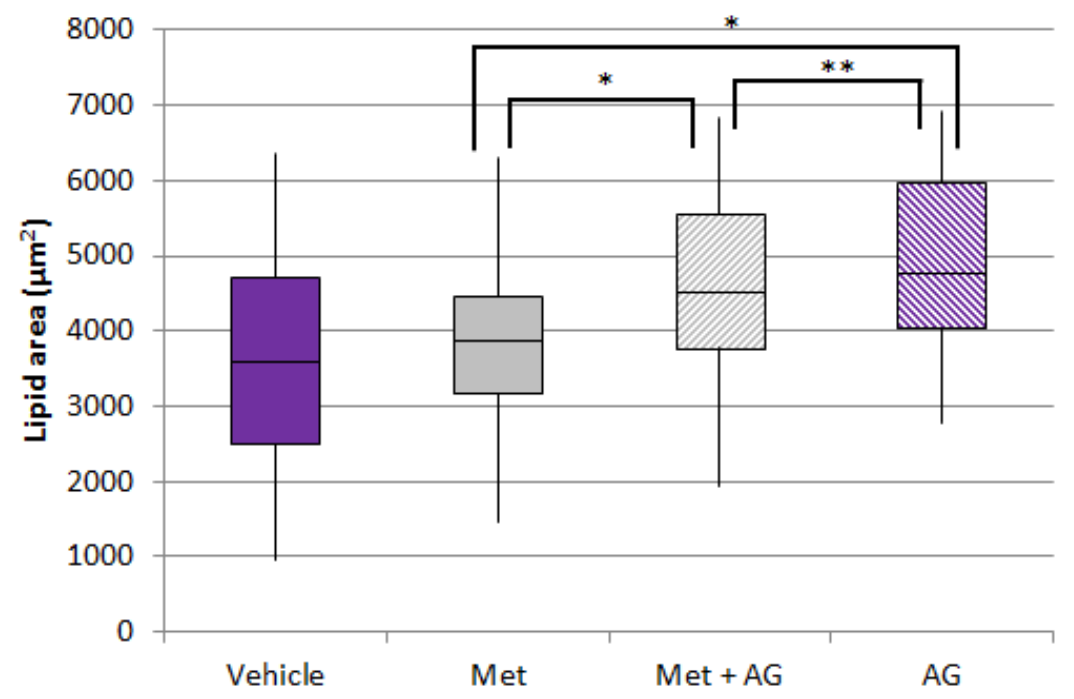

Figure 5.4. Box plot of interquartile range and median intracellular lipid area within vehicle, acylghrelin, metformin and metformin plus acyl-ghrelin treated cells in a hyperglycaemic environment. Statistical analysis was conducted using non-parametric measures ( $n=600$ per treatment). The Kruskal Wallis test determined statistical significance between groups which are indicated by * for $\mathrm{p}<0.001$ and $^{* *}$ for $\mathrm{p}<0.05$. Metformin (Met), acyl-ghrelin (AG) and metformin/acyl-ghrelin (Met + $A G)$. 


\subsubsection{Real Time gene expression}

\section{Fold change relative to vehicle}

When gene expression data was analysed with respect to combined metformin and acyl-ghrelin exposure against a vehicle control there was a significant down regulation of $L X R B$ with a $2.9( \pm 0.7)$ fold change $(p<0.01)$, a significant up regulation of SREBF1 with a $4.9( \pm 1.4)$ fold change $(p<0.01)$ and a non-significant change in expression of $A B C G 1$ of $1.1( \pm 0.02)$ fold change $(p=0.36)$ (Table 5.1$)$.

\section{Fold change relative to metformin}

Exposure to a combination of metformin and acyl-ghrelin caused a significant down regulation of two genes; $A B C G 1$ with $34.2( \pm 4.0)$ fold change and $L X R B$ with 35.1 $( \pm 0.9)$ fold change, both $p<0.01$. However, there was no fold change difference for SREBF1 between the treatment two treatment groups $(p=0.08)$ (Table 5.1).

\section{Fold change relative to acyl-ghrelin}

Furthermore, when combined metformin and acyl-ghrelin exposure was compared to acyl-ghrelin only exposure, data indicated that there was a significant effect of metformin with down regulation of $3.5( \pm 0.4)$ fold change for $L X R B$, a significant up regulation of $2.5(0.2)$ fold change for $A B C G 1$, and a significant up regulation of 3.5 $( \pm 0.5)$ fold change for SREBF1 (Table 5.1). 


\begin{tabular}{|c|c|c|c|c|}
\hline Group & Gene of interest & Fold Change & Direction & P-value \\
\hline \multirow{2}{*}{ V vs } & LXRB & $10.1(1.2)$ & & $<0.01$ \\
\hline & $A B C G 1$ & $1.1(0.02)$ & $\longleftrightarrow$ & 0.36 \\
\hline Met+AG & SREBF1 & $4.9(1.4)$ & 4 & $<0.01$ \\
\hline & LXRB & $34.2(4.0)$ & | & $<0.01$ \\
\hline & ABCG1 & $35.1(0.9)$ & & $<0.01$ \\
\hline Met+AG & SREBF1 & $0.7(0.2)$ & $\longleftrightarrow$ & 0.08 \\
\hline \multirow{2}{*}{$A G$ vs } & LXRB & $3.5(0.4)$ & 1 & $<0.01$ \\
\hline & ABCG1 & $2.5(0.2)$ & 4 & $<0.01$ \\
\hline Met+AG & SREBF1 & $3.5(0.5)$ & 4 & $<0.01$ \\
\hline
\end{tabular}

Table 5.1. Relative fold change values for gene expression data in vehicle, acyl-ghrelin and dual metformin/acyl-ghrelin treated cells in hyperglycaemic environment at 17 hours. Fold change between -1.5 and 1.5 is classed as no relative gene expression change. Standard deviation between $\Delta \Delta C$ t of experimental repeats are shown in brackets $(n=6)$. Statistical difference of $\Delta C t$ in comparison group were determined using an independent t-test, statistically significant values shown in bold. Vehicle (V), Acyl-ghrelin (AG), Metformin (Met) and Metformin/Acyl-ghrelin (Met + AG).

$\uparrow$ - up regulation. $\downarrow$-down regulation. $\longleftrightarrow$-no fold change, 


\subsubsection{Adipokine levels}

The quantity of IL-10 present within the cellular supernatant did not significantly change across all four treatment groups $(p=0.70)$ (Table 5.2).

\begin{tabular}{|c|c|c|c|}
\cline { 2 - 4 } \multicolumn{1}{c|}{} & \multicolumn{3}{c|}{ IL-10 $[\mathrm{pg} / \mathrm{mL}]$} \\
\hline Treatment & No acyl-ghrelin & Acyl-ghrelin & P-Value \\
$(\mathrm{n}=6)$ & $(\mathrm{n}=6)$ & \\
\hline Vehicle & 258.2 & 282.8 & 0.39 \\
$(\mathrm{n}=6)$ & {$[245-283]$} & {$[224-319]$} & \\
\hline Metformin & 282.5 & 257.4 & 1.00 \\
(n=6) & {$[252-300]$} & {$[172-316]$} & \\
\hline P-Value & 0.44 & 0.90 & \\
\hline
\end{tabular}

Table 5.2. IL-10 levels for all treated cells in hyperglycaemic environment. Statistical analysis conducted using non-parametric parameters using a Kruskal Wallis test.

\section{Compared to vehicle- IL-6}

When combined metformin and acyl-ghrelin treatment was compared to a PBS vehicle control there was a significant decline in IL-6 levels (Met+AG v Veh; 12.6 $[9.0-16.6] \mathrm{pg} / \mathrm{mL}$ v $89.5[87.7-95.5] \mathrm{pg} / \mathrm{mL} ; \mathrm{p}<0.05)$ (Figure 5.5).

\section{Compared to metformin- IL-6}

When compared to only metformin treated cells combined metformin and acylghrelin treatment caused a significant decline in IL-6 levels. (Met + AG v Met; 12.6 $[9.0-16.6] \mathrm{pg} / \mathrm{mL} v 23.2[19.3-25.6] \mathrm{pg} / \mathrm{mL} ; \mathrm{p}<0.05)$ (Figure 5.5). 


\section{Compared to acyl-ghrelin-IL-6}

Dual treatment also caused a significant decline in the level of IL-6 within the cell supernatants in comparison to acyl-ghrelin only treatment (Met + AG v AG; 12.6 $[9.0-16.6] \mathrm{pg} / \mathrm{mL}$ v $75.5[68.9-89.5] \mathrm{pg} / \mathrm{mL} ; \mathrm{p}<0.05)$ (Figure 5.5).

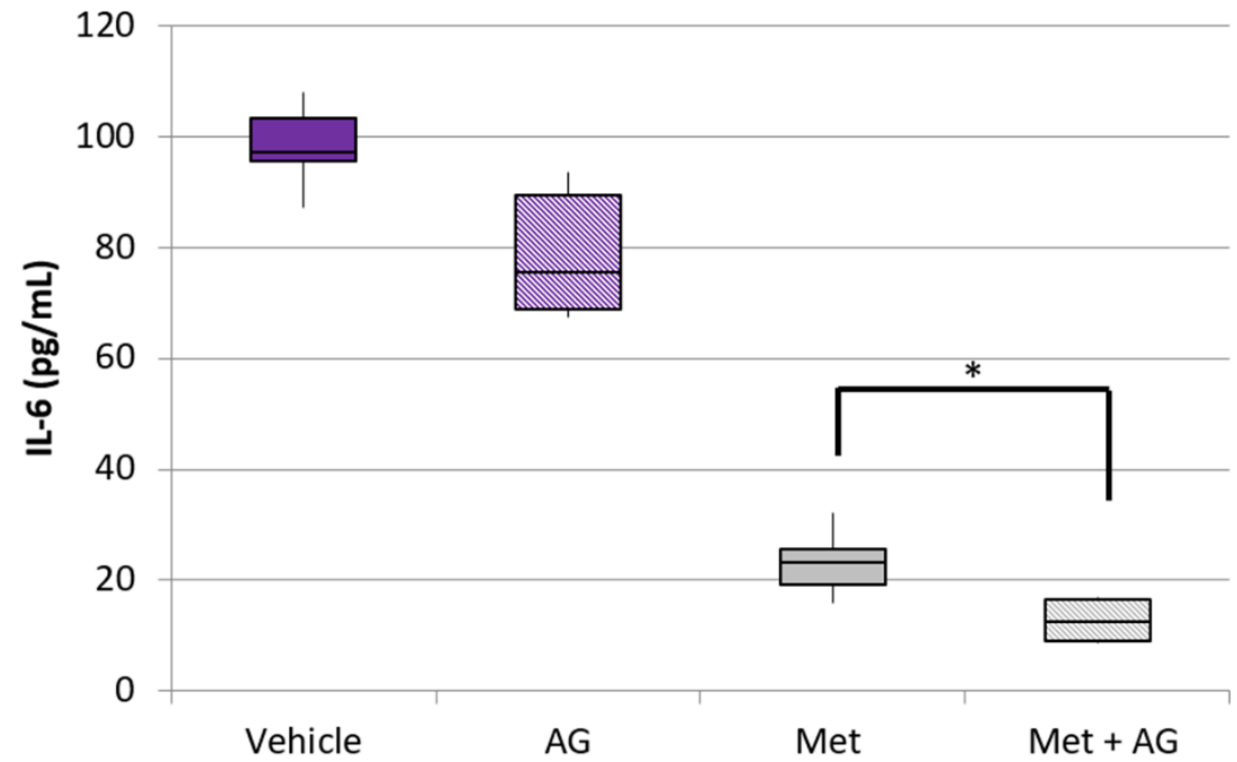

Figure 5.5. Box plot of IL-6 levels within vehicle, acyl-ghrelin, metformin and metformin/acyl-ghrelin treated cells in hyperglycaemic environment. Statistical analysis was conducted using non parametric measures $(n=6)$. The Kruskal Wallis test determined statistical significance $(p<0.05)$ between groups which are indicated by *

Acyl-ghrelin (AG), Metformin (Met) and Metformin/Acyl-ghrelin (Met + AG) 


\subsection{Discussion}

\subsubsection{Metformin and lipid retention}

Metformin mono therapy exhibited minimal effect on mature adipocyte intracellular lipid area, however there was an increase in the expression of the key lipid retention genes $A B C G 1, \angle X R B$ and SREBF1. This data suggests that metformin can act to alleviate lipid retention within cells through promotion of cellular lipid export. However 17 hours may not be a long enough duration to exhibit this effect on a cell morphological level. In addition to this, metformin is shown to have an effect on acyl-ghrelin mediated lipid retention, resulting in the curtailment in acylghrelin's promotion of lipid storage within mature adipocytes.

Previous literature supports this hypothesis, demonstrating that metformin treatment of THP-1 macrophages results in the promotion of cholesterol efflux and expression of both $A B C A 1$ and $A B C G 1$, this promotion however was deemed to be independent of $L X R$ expression (Luo et al., 2017). Metformin stimulation of ABCG1 is thought to be the result of an increase in the expression of fibroblast growth factor (FGF) 21 within adipose tissue and the liver (Luo et al., 2016). Furthermore, my data indicate that the action of metformin on $A B C G 1$ is present within cells pretreated with acyl-ghrelin or with a vehicle control, indicating that metformin is counteracting acyl-ghrelin's down regulation of $A B C G 1$, resulting in no overall change in expression upon dual treatment. In addition to these findings, I determined that the effect on ABCG1 expression is independent of $L X R B$ within metformin treated human adipocytes, which is in line with published data that indicates that activation of AMPK within mouse macrophages stabilizes the mRNA expression of $A B C G 1$ in a LXR-independent manner (Li et al., 2010a). 
Metformin administration is shown to decrease hepatic lipogenesis via its partial mitochondrial uncoupling effect, which activates AMPK and inhibits SREBP1c transcriptional activities (Ferre and Foufelle, 2007, Zhou et al., 2001). However, in my data, metformin exposure caused an increase in SREBF1 expression. This increase in SREBF1 expression was seen in experimental conditions, whether cells were pre-exposed or not to acyl-ghrelin. I hypothesise this paradoxical result is due to a depot specific action of SREBF1 within adipocytes, resulting in AMPK regulatory action also being depot specific and not true of a hyperglycaemic adipocyte cell depot.

The action of AMPK, described above, on SREBP-1c is due to AMPK directly inhibiting ligand-induced LXR activity in addition to blocking the production of endogenous LXR ligands within hepatic loci (Yap et al., 2011). This chapter indicates a marked increase in $L X R B$ and SREBF1 expression within an adipose depot, signifying alternative actions dependant on experimental depot. However, previous studies have shown that metformin administration coincides with an increase in GLUT4 expression within human skeletal muscle cells (Al-Khalili et al., 2005), and within subcutaneous adipose tissue of women with polycystic ovary syndrome (Jensterle et al., 2008). GLUT4 and LXR share an intrinsic pathway with GLUT4 being up regulated by LXR agonists (Calkin and Tontonoz, 2012). One possible explanation may be that within a hyperglycaemic state, metformin action on mature adipocytes increases GLUT4 expression via LXR up regulation. 


\subsubsection{Metformin and inflammation}

Metformin exposure had no effect on the anti-inflammatory marker IL-10, alone or in combination with acyl-ghrelin. Previously published data in murine macrophages reports that metformin induces an anti-inflammatory response under LPS immunechallenge (Hyun et al., 2013, Kelly et al., 2015, Zhou et al., 2016). However, previous work was conducted within murine models and could account for variation in findings that may be the result of a species-specific effect.

In contrast to IL-10, our IL-6 data are supported by previously published work showing that metformin supresses the expression and release of IL-6 (Zhou et al., 2016, Kelly et al., 2015). This protective effect in the inflammatory state, via a decline in IL-6, is also present when mature human adipocytes are pre-exposed to acyl-ghrelin, indicating metformin further enhances the suppression of proinflammatory cytokines that are present following exposure to acyl-ghrelin.

Metformin exposure was shown to supress the expression of proinflammatory cytokines through cellular regulation via AMPK signalling in rodents, resulting in induced NFKB inhibition and enhanced expression of anti-inflammatory cytokines via AMPK activation. This response was shown to also occur in the presence of AGEs, indicating metformin also inhibits AGE-induced inflammatory response (Zhou et al., 2016). In addition to cellular mechanisms, circulatory markers of inflammation are also shown to be reduced when humans are treated with metformin, including the suppression of neutrophil to lymphocyte ratio (Cameron et al., 2016), indicating that metformin acts to reduce the inflammatory state in those with or without T2D, an action that is accentuated in the presence of acylghrelin. 


\subsection{Conclusion}

Published studies show an increased level of acyl-ghrelin present within obesity and T2D which may have a detrimental effect on cellular lipid retention, this effect could be diminished via metformin administration. With respects to an inflammatory response, it is clear that co-administration of metformin and acylghrelin could have therapeutic advantages in reducing the presence of proinflammatory adipokine levels in hyperglycaemia. Expansion and investigation into this interlinked modulation of the inflammatory state could reveal key therapeutic pathways.

\subsection{Limitations}

The experimental design within this chapter was based on that of previous chapter findings, however, it was limited to only one time point at 17 hours. Within future work, an expansion on this chapter could include the analysis of treatment exposure at a greater frequency i.e. baseline to 17 hours with hour intervals to establish a response curve over time. In addition to this, dosage concentrations were based on previous literature rather than the physiological range present within circulation, therefore, a dose response curve would also have been beneficial. However, due to time constraints this was not conducted within this chapter, as the aim was to establish whether cell dosage could contribute to a change and if this change could be sufficient to allow for further exploration. 
Chapter 6

General Discussion 
The aim of this project was to translate previous findings present within murine models for an intrinsic role played by acyl-ghrelin on lipid retention within adipose tissue, and whether this acyl-ghrelin associated lipid retention could alter the inflammatory state within those that are obese and/or have T2D (Davies et al., 2009, Miegueu et al., 2011). This thesis on the based analysis of expression levels of specific lipid retention genes and inflammatory markers within an adipocyte cell line and ex vivo hVAT samples from individuals that are obese and/or have T2D, to identify whether acyl-ghrelin has a mediatory role that can be explored as a possible therapeutic target for $\mathrm{T} 2 \mathrm{D}$ and related comorbidities. Furthermore, to expand on in vitro and ex vivo data collected in Chapter 3 and 4, application of acylghrelin in response to the first line drug therapy of T2D, metformin was also investigated.

Within Chapter 3, exposure to acyl-ghrelin in a normoglycaemic state resulted in an increase in lipid retention via the initial down regulation of $A B C G 1$. Over time, this effect was eliminated via a compensatory increase in LXRB expression, leading to an up regulation of $A B C G 1$, returning it to a level seen within vehicle treated cells (Figure 6.1). In Chapter 4, circulating acyl-ghrelin concentrations were at their highest in non-obese individuals, however acyl-ghrelin mediated lipid retention shown in vitro was not mirrored in lipid retention markers of the ex vivo samples. This may indicate that lipid retention within visceral adipose tissue of non-obese individuals is independent of circulatory acyl-ghrelin levels. In vitro experiments required the exposure of mature adipocytes directly to acylghrelin, whereas circulatory levels present within individuals may not be representative of the physiological level of acyl-ghrelin present within adipose 
tissue. Thus a comparison between the two must be under taken with caution as acyl-ghrelin's action may be depot/cell specific (Miegueu et al., 2011, Porteiro et al., 2013, Churm et al., 2017, Demers et al., 2009).

Over the time span of the initial experiments SREBF1 also showed an increase in expression levels within acyl-ghrelin treated mature adipocytes in a normoglycaemic state. This is in line with previous literature that shows SREBF1 is a target for LXR $\alpha / \beta$ ligands (Repa et al., 2000). Therefore, these data suggest that SREBF1 regulation is dependent on $L X R$ up regulation rather than a direct effect of acyl-ghrelin. My results suggest that the intracellular lipid area increase was due to the depot specific action of acyl-ghrelin on the down regulated ABCG1 expression, and that over time, within a normoglycaemic state the cell aims to counteract this effect via up regulation of $L X R$ and the resultant up regulation of $A B C G 1$. However, this counteraction also results in the up regulation of SREBF1 independent of acylghrelin exposure. This hypothesis corresponds with results from the ex vivo samples, which showed no association within the total sample cohort between LXRB or SREBF1 expression and GHRL expression or circulating acyl-ghrelin levels.

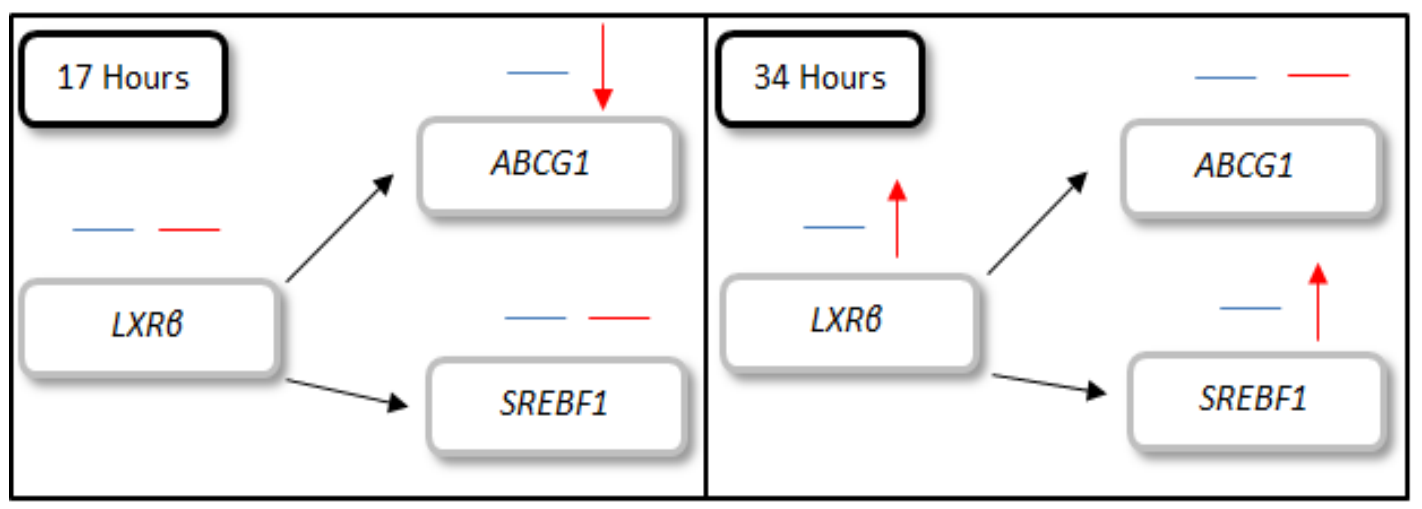

Figure 6.1. Gene expression profile over time (17 and 34 hours) of SGBS cells within normoglycaemic environment treated with either acyl-ghrelin (effect shown in red) or vehicle (effect shown in blue). 
Upon evaluation of acyl-ghrelin mediation of the lipid retention pathway within mature adipocytes in a hyperglycaemic environment there was deviation in the established pathway compared to that shown for normoglycaemia (Figure 6.2). Within human adipocytes an immediate effect was evident when exposed to acylghrelin, producing a significant increase in lipid area, via the down regulation of both $L X R B$ and $A B C G 1$. Therefore, one hypothesis is that in addition to acyl-ghrelin's effect on the down regulation of $A B C G 1$, the cumulative effect of a hyperglycaemic environment resulted in the further down regulation of $A B C G 1$ and an impingement of the cells counter measure via $L X R B$ by causing its down regulation instead (Xue et al., 2009), producing a dual mediation of lipid retention via an inhibition of the cholesterol export mechanism.

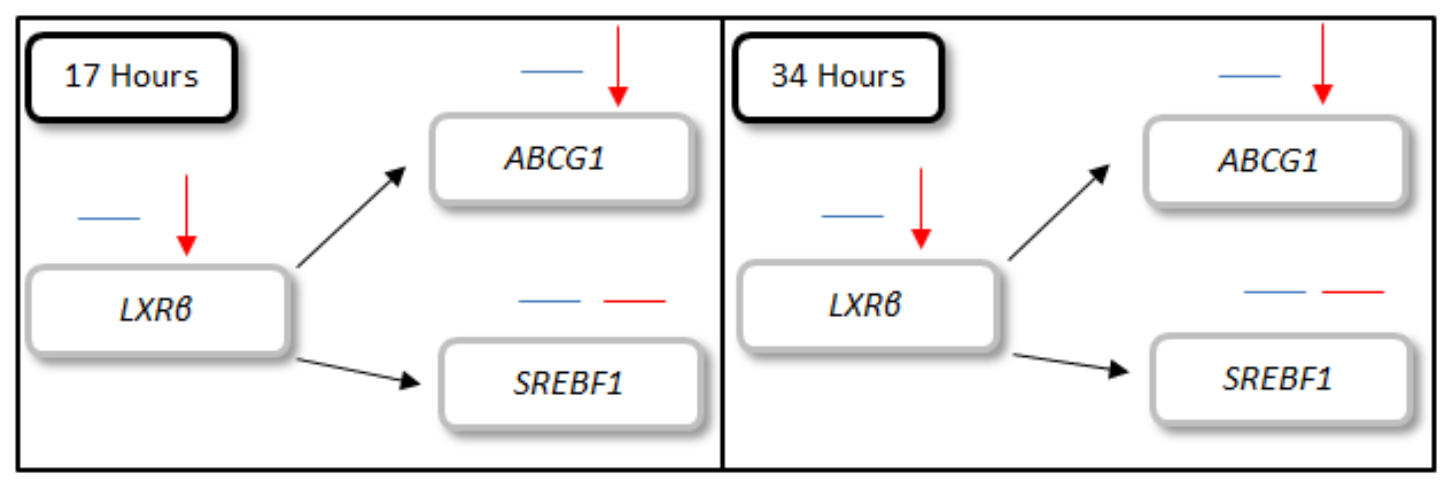

Figure 6.2. Gene expression profile over time (17 and 34 hours) of SGBS cells within hyperglycaemic environment treated with either acyl-ghrelin (effect shown in red) or vehicle (effect shown in blue).

Furthermore, ex vivo experiments in Chapter 4 also demonstrated an association with hyperglycaemic environment with LXRB within the hVAT of those with T2D. Due to circulating acyl-ghrelin levels being significantly reduced in individuals with $\mathrm{T} 2 \mathrm{D}, \mathrm{I}$ hypothesise that acyl-ghrelin action on $\mathrm{T} 2 \mathrm{D}$ adipose tissue is 
minimal, suggesting that the down regulation of $A B C G 1$ and SREBF1 in the OT2D group was the result of diminished LXR promotion via the presence of an elevated blood glucose rather than acyl-ghrelin itself (Mitro et al., 2007, Mauerer et al., 2009). Further evidence of acyl-ghrelins' diminished action within T2D is the negative correlation between plasma fasting glucose, GHRL mRNA expression and acyl-ghrelin levels, indicating that ghrelin levels within T2D are under glucose regulation. As previously discussed in Chapter 4, the ratio of acyl-ghrelin, total ghrelin and desacyl-ghrelin within the metabolic state is controversial across published study cohorts (Rodríguez et al., 2012, Dardzińska et al., 2014, Supák et al., 2016). This ex vivo investigation of the ghrelin axis suggest that acyl-ghrelin concentrations may be dependent on GHRL expression only, and not its posttranslational modification, accounting for a lower plasma acyl-ghrelin level seen in this cohort, and reduced desacyl-ghrelin level often reported within the metabolic state (Rodríguez et al., 2012).

VAT samples from OT2D group showed an alternative relationship between hyperglycaemia and lipid biosynthesis gene expression. SREBF1 was down regulated in ex vivo samples, but had no fold change in mature adipocytes. This could be due to two hypothesies: (i) depot-specific action and/or (ii) interaction with antidiabetic therapeutic agents. A depot-specific effect would allow for variability to stem from the vast cellular make up of adipose tissue in contrast to the pure mature adipocytes culture. Adipose tissue has been reported to be affected by the presence of a hyperglycaemic environment and acyl-ghrelin by impacting macrophages (Liu et al., 2012), monocytes (Dixit et al., 2004) and T cells (Dixit et al., 2004). As these cell types were not investigated in co-culture with adipocytes we 
are unable to determine whether there is an alternative effect occurring within ex vivo samples resulting in a variation in data. The second theory has been discussed in detail within Chapter 5, where metformin was prescribed and taken by $90 \%$ of the individuals recruited for this study with T2D, therefore impact on adipocyte lipid retention was examined. In contrary to published data within murine models (Zhou et al., 2001, Lin et al., 2000), metformin caused an increase in SREBF1 transcription within mature adipocytes, therefore this variation within a hyperglycaemic state could be due to the use of a homogeneous cell source (mature adipocytes) rather than a heterogeneous animal model.

Furthermore, combined treatment with both acyl-ghrelin and metformin suggested that metformin altered the expression of both LXRB and SREBF1; increasing expression of SREBF1, whilst down regulating $L X R B$. This demonstrates that metformin's action is independent of LXR stimulation, diminishing its action on $L X R$ expression in the presence of both hyperglycaemia and high acyl-ghrelin. Additionally, metformin treatment produced a significant up regulation of $A B C G 1$, however upon administration of combined acyl-ghrelin and metformin there was no change in $A B C G 1$ expression compared to vehicle treated cells, indicating that metformin up regulation of $A B C G 1$ counteracts the effects of acyl-ghrelin exposure.

Obesity is often characterised by an increase in the prevalence of dyslipidaemia, with the main factor often being uncontrolled fatty acid lipolysis from VAT, which is dependent upon adipocyte size and number of ATM (Gutierrez et al., 2009). This study demonstrates an association between acyl-ghrelin with both lipid retention genes and plasma lipid markers, indicating that acyl-ghrelin levels are a risk factor associated with dyslipidaemia. 
Acyl-ghrelin's ability to mediate lipid retention led to the hypothesis that it would also result in the promotion of a pro-inflammatory state. Acyl-ghrelin exposure in vitro caused a significant decline in IL-6 in both normo- and hyperglycaemic environments, which coincides with findings in murine models (Baatar et al., 2011, Chorny et al., 2008, Wu et al., 2008) and an increase in the antiinflammatory marker IL-10 in a normoglycaemic environment. However, within a hyperglycaemic environment this anti-inflammatory IL-10 promotion via acylghrelin was diminished. Complementary to in vitro work, analysis of ex vivo samples showed that mRNA levels of GHRL were significantly positively associated with IL-10 within the total sample cohort and TAOS (\%) within an obese cohort. This association was lost within the T2D cohort indicating that, as the in vitro arm of the experiments, glucose may be diminishing acyl-ghrelin's effect on oxidative burden and anti-inflammatory state. IL-10 levels across the chapters provided contradictory findings with respect to ghrelin association, however it is important to note that an association of GHRL expression within ex vivo sample may indicate two alternative theories. Firstly, acyl-ghrelin does promote anti-inflammatory state markers (Waseem et al., 2008), however this effect does not take place within adipocytes, instead being from an alternative cell source. Within the immune system, acylghrelin has been shown to induce an anti-inflammatory state through $M 2$ macrophage polarization and inhibiting Th1 cells and increasing the polarization of both Th2 and T cells (Pereira et al., 2017). Secondly, as previously discussed, increases in GHRL mRNA levels could indicate an increase in either or both acyl-and desacyl-ghrelin. Desacyl-ghrelin, which was once considered biologically inactive, is 
now known to play a role in various metabolic pathways, including that of macrophage polarization (Pereira et al., 2017, Au et al., 2017)

Obesity and T2D are associated with an increase in IL-6 as well as a decline in both plasma IL-10 (van Exel et al., 2002, Esposito et al., 2003) and TAOS levels (Fenkci et al., 2003, Prior et al., 2017). The combination of both in vitro and ex vivo data presented within the previous chapters indicate that acyl-ghrelin may play a beneficial role abrogating inflammation, and that exploration of this novel pathway within human adipocytes could elucidate key therapeutic targets for the management of low-grade inflammation.

When metformin treatment was analysed in Chapter 5, it was shown to have no effect on the pro-inflammatory marker IL-10, nor when used in combination with acyl-ghrelin. However, the combination of the two did cause a significant decrease in IL-6 compared to either treatment alone. In addition to this, combination of metformin and acyl-ghrelin alleviated the detrimental effect of acylghrelin on lipid retention. Therefore, co-administration within adipocytes resulted in the promotion of inflammatory health, but with no effect on cellular lipid retention.

I hypothesise that the mode in which acyl-ghrelin acts on both lipid retention and inflammatory response is similar to that of metformin, acting via AMPK. Acyl-ghrelin has been shown to directly activate neuropeptide $Y$ (NPY) neurons in the ARC via an increase in AMPK-mediated signalling (Kohno et al., 2008, Kang et al., 2015), with AMPK induction inhibiting the release of IL-6 and other proinflammatory cytokines via the attenuation of NFKB (Lihn et al., 2008). Furthermore, due to metformin's well described action on AMPK (Phoenix et al., 
2009), combination treatment may exhibit a dual activation of AMPK activity which would amplify this effect.

However, contradictory to this theory is acyl-ghrelin's action on lipid retention. In contrast to the AMPK-inflammation relationship, this does not fit the project's findings, as AMPK activation results in the alleviation of lipid retention and promotion of cholesterol efflux due to the up regulation of $A B C G 1$ (Li et al., 2010b) and the inhibition of SREBF1 (Pedram et al., 2013). Therefore, further work is required to elucidate the cellular mechanisms in which acyl-ghrelin acts to mediate lipid retention within adipocytes.

This work translated previous murine findings that acyl-ghrelin and glycaemic levels are detrimental to lipid retention causing an increase in intracellular lipid area via alterations within the LXR-ABC pathway and the independent action acyl-ghrelin has on the inflammatory state. However, acylghrelin was also shown to be beneficial to inflammatory health via a reduction in pro-inflammatory marker IL-6. Therefore, the combination of acyl-ghrelin and metformin treatment could allow for a reduction in low-grade inflammation present within the adipocytes of individuals with T2D, thus ameliorating insulin resistance and improving insulin sensitivity. 


\section{Limitations \& future work}

The main methodological limitations are set out and discussed in each chapter (sections 3.7, 4.7 and 5.7). However, in expansion to these the project design as a whole was also restricted by general methodology limitations that could have enhanced analysis. For example, relative mRNA changes provided crucial understanding of acyl-ghrelin's effect at a gene level, deducing significant fold changes present when comparing groups or treatments. However, this does not allow for the quantification of mRNA transcript, nor whether this is translated at a protein level. Further analysis via Western blot could allow for greater insight into cellular changes and the degree in which acyl-ghrelin exhibits a mediatory role.

As noted within Chapter 4, exploratory analysis associated the expression of GHRL with key inflammatory markers, yet this encompasses acyl-ghrelin in addition to desacyl-ghrelin. This project would have benefitted if time allowed, for the further analysis of desacyl-ghrelin dosing within in vitro experiments to act as a comparator to the cellular responses to acyl-ghrelin.

Within the project proposal, an adequate power calculation deemed that 30 ex vivo samples would suffice to elucidate any statistical significance within the data. However, in reality the analysis of small numbers of samples proved difficult, especially to elucidate significance within the study data. Due to sample collections being from routine non-emergency abdominal surgeries, often the collection of samples would be delayed due to surgery cancellations. Another minor limitation within this study was the ability to only look at the interaction of acyl-ghrelin with one type of T2D therapy. Further information on acyl-ghrelin's potential to 
ameliorate the inflammatory response may have been yielded from exposure to further combinations, such as insulin analogues or GLP-1 agonist therapies.

Despite the limitation of sample size, the data provide a novel insight into an area of expertise that still has several knowledge gaps. Even though this thesis pinpoints specific pathways in which acyl-ghrelin interacts with cellular energy balance, an expansion should aim to yield the exact pathway in which acyl-ghrelin results in alterations in lipid retention and inflammation. This project demonstrates that under the correct circumstances acyl-ghrelin can be used as a therapeutic target for T2D, however the way this is optimised for maximum impact and minimum consequence requires investigating. 
Appendix 1

Ethical approval

\& patient information sheet 


\section{EoSRES}

East of Scotland Research Ethics Service (EoSRES) REC 1

Tayside Medical Sciences Centre (TASC)

Residency Block C, Level 3

Ninewells Hospital \& Medical School

George Pirie Way

Dundee DD1 9SY
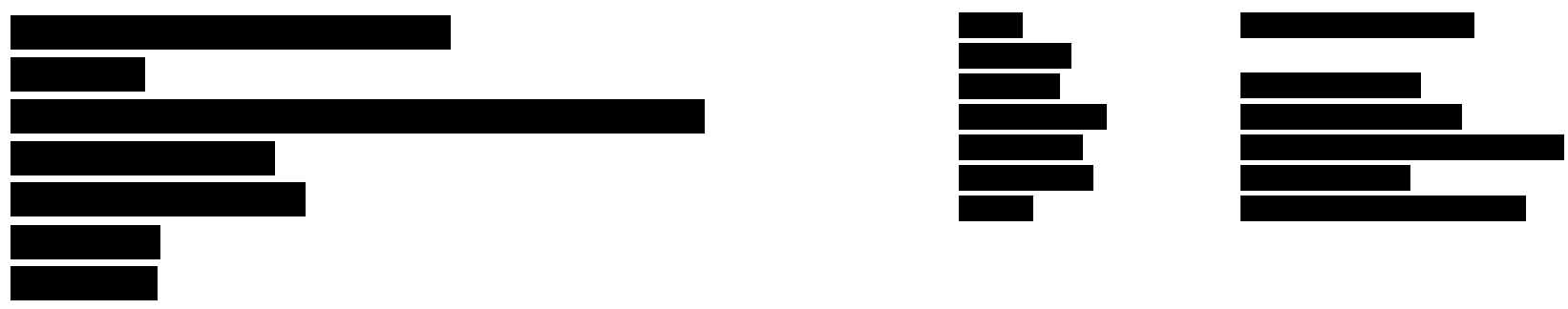

Dear

Study title:

Ghrelin mediated lipid retention and insulin resistance in human visceral adipose tissue

REC reference: IRAS project ID:

14/ES/1073

163151

Thank you for your letter of $16^{\text {th }}$ September 2014, responding to the Proportionate Review Sub-Committee's request for changes to the documentation for the above study.

The revised documentation has been reviewed and approved by the sub-committee.

We plan to publish your research summary wording for the above study on the NRES website, together with your contact details, unless you expressly withhold permission to do so.

Publication will be no earlier than three months from the date of this favourable opinion letter. Should you wish to provide a substitute contact point, require further information, or wish to withhold permission to publish, please contact the REC Manager

eosres.tayside@nhs.net.

\section{Confirmation of ethical opinion}

On behalf of the Committee, I am pleased to confirm a favourable ethical opinion for the above research on the basis described in the application form, protocol and supporting documentation as revised.

- $\quad$ The Committee asked the researcher to consider putting in a substantial amendment if they would like to consider retaining blood \& tissue samples for future research.

\section{Conditions of the favourable opinion}

The favourable opinion is subject to the following conditions being met prior to the start of the study.

Management permission or approval must be obtained from each host organisation prior to the start of the study at the site concerned. 
Management permission ("R\&D approval") should be sought from all NHS organisations involved in the study in accordance with NHS research governance arrangements.

Guidance on applying for NHS permission for research is available in the Integrated Research Application System or at http://www.rdforum.nhs.uk.

Where a NHS organisation's role in the study is limited to identifying and referring potential participants to research sites ("participant identification centre"), guidance should be sought from the $R \& D$ office on the information it requires to give permission for this activity.

For non-NHS sites, site management permission should be obtained in accordance with the procedures of the relevant host organisation.

Sponsors are not required to notify the Committee of approvals from host organisations.

\section{$\underline{\text { Registration of Clinical Trials }}$}

All clinical trials (defined as the first four categories on the IRAS filter page) must be registered on a publically accessible database within 6 weeks of recruitment of the first participant (for medical device studies, within the timeline determined by the current registration and publication trees).

There is no requirement to separately notify the REC but you should do so at the earliest opportunity e.g. when submitting an amendment. We will audit the registration details as part of the annual progress reporting process.

To ensure transparency in research, we strongly recommend that all research is registered but for non-clinical trials this is not currently mandatory.

If a sponsor wishes to contest the need for registration they should contact

, the HRA does not, however, expect exceptions to be made. Guidance on where to register is provided within IRAS.

It is the responsibility of the sponsor to ensure that all the conditions are complied with before the start of the study or its initiation at a particular site (as applicable).

\section{Ethical review of research sites}

The favourable opinion applies to all NHS sites taking part in the study, subject to management permission being obtained from the NHS/HSC R\&D office prior to the start of the study (see "Conditions of the favourable opinion" above).

\section{Approved documents}

The documents reviewed and approved by the Committee are:

\begin{tabular}{|l|l|l|}
\hline Document & Version & Date \\
\hline $\begin{array}{l}\text { GP/consultant information sheets or letters [GP letter, V1, } \\
\text { 20th Aug 2014 (if needed)] }\end{array}$ & Version 1 & 20 August 2014 \\
\hline IRAS Checklist XML [Checklist_17092014] & & 17 September 2014 \\
\hline Other [Email with contact number ] & & 04 September 2014 \\
\hline Other [Reply to panel comments] & Version 1 & 16 September 2014 \\
\hline $\begin{array}{l}\text { Participant consent form [Consent form, V2, 16th Sept 2014 } \\
\text { highlighted] }\end{array}$ & Version 2 & 16 September 2014 \\
\hline
\end{tabular}




\begin{tabular}{|c|c|c|}
\hline Participant consent form [Consent form, V2, 16th Sept 2014] & Version 2 & 16 September 2014 \\
\hline Participant information sheet (PIS) [PIS, V2, 16th Sept 2014] & Version 2 & 16 September 2014 \\
\hline $\begin{array}{l}\text { Participant information sheet (PIS) [PIS, V2, 16th Sept } 2014 \\
\text { highlighted] }\end{array}$ & Version 2 & 16 September 2014 \\
\hline REC Application Form & & 01 September 2014 \\
\hline $\begin{array}{l}\text { Research protocol or project proposal [NISCHR Successful } \\
\text { application including proposal] }\end{array}$ & Version 1 & 20 August 2014 \\
\hline $\begin{array}{l}\text { Summary CV for Chief Investigator (CI) [CV Jeffrey Stephens, } \\
\text { August 2014] }\end{array}$ & Version 1 & 20 August 2014 \\
\hline Summary CV for student [CV Rachel Churm, PhD Student] & Version 1 & 20 August 2014 \\
\hline $\begin{array}{l}\text { Summary CV for supervisor (student research) [CV Sarah } \\
\text { Prior, Academic supervisor] }\end{array}$ & Version 1 & 20 August 2014 \\
\hline
\end{tabular}

\section{Statement of compliance}

The Committee is constituted in accordance with the Governance Arrangements for Research Ethics Committees and complies fully with the Standard Operating Procedures for Research Ethics Committees in the UK.

\section{After ethical review}

\section{$\underline{\text { Reporting requirements }}$}

The attached document "After ethical review - guidance for researchers" gives detailed guidance on reporting requirements for studies with a favourable opinion, including:
Notifying substantial amendments
Adding new sites and investigators
Notification of serious breaches of the protocol
Progress and safety reports
Notifying the end of the study

The HRA website also provides guidance on these topics, which is updated in the light of changes in reporting requirements or procedures.

\section{Feedback}

You are invited to give your view of the service that you have received from the National Research Ethics Service and the application procedure. If you wish to make your views known please use the feedback form available on the HRA website: http://www.hra.nhs.uk/about-the$\underline{\text { hra/governance/quality-assurance }}$

We are pleased to welcome researchers and R \& D staff at our NRES committee members' training days - see details at http://www.hra.nhs.uk/hra-training/ 


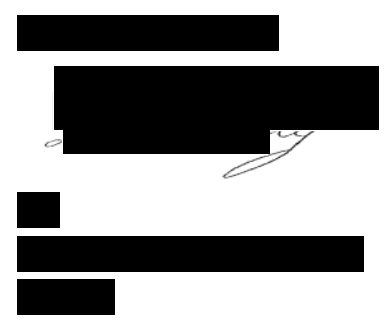

Enclosures:

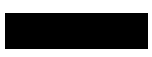

"After ethical review - guidance for researchers"

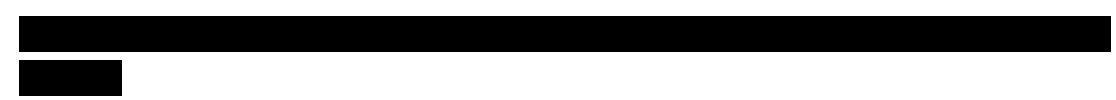


Department of Diabetes and Endocrinology Morriston Hospital, Morriston, Swansea, SA6 6NL

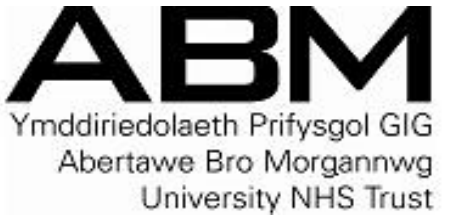

Tel: 01792704078

\title{
Fat and its role in obesity and diabetes Patient Information Sheet
}

\begin{abstract}
You are being invited to take part in a research study that is being undertaken as part of an educational qualification, with the goal of improving our knowledge of why human fat is important in obesity and diabetes. The purpose is to improve our knowledge of hormones and chemicals made in the fat within the abdomen and how these might be important in ill-health related to diabetes.
\end{abstract}

Before you decide, it is important to understand why the research is being done and what it will involve. Please take time to read the information carefully. Talk to others about the study if you wish. Ask us if there is anything that is not clear or if you would like to know more. Part $\mathbf{1}$ tells you the purpose of this study and what will happen to you if you take part. Part 2 gives you more information about the conduct of the study. Take time to decide whether or not you wish to take part.

\section{Part 1}

\section{What is the purpose of this study?}

Obesity (carrying too much weight) and type 2 diabetes are major health concerns and contribute to heart disease and general ill health. Fat from inside the abdomen (visceral fat) appears to be important in causing these conditions. This research looks at the differences between abdominal fat from people who are lean, obese or have type 2 diabetes. We will look at differences in the size and development of fat cells and also on proteins and hormones that are produced by fat from people who are lean, obese or have type 2 diabetes. We will also examine how genes within fat might influence various chemicals produced in fat. The blood sample will be used to measure levels of molecules with the body that help reduce oxidative stress. Eventually the work may lead to a better understanding of why too much fat causes diabetes and heart disease. This might lead to newer treatments to prevent and treat these common conditions.

\section{Why have I been invited to take part?}

You have been asked to take part in this study because you are due to have an operation on your abdomen. As part of the operation the surgeon will routinely remove or move around abdominal fat. We are asking if you would be happy to donate a small piece of this fat, along with a blood sample, for the research study. We are asking patients who are lean, obese or have type 2 diabetes to be involved.

\section{Do I have to take part?}

Your participation in this study is entirely voluntary. It is up to you to decide whether or not to take part. We will describe the study and go through this information sheet which you will keep. We will then ask you to sign a consent form to show you have agreed to take part. The original signed and dated copy will remain at the hospital and you will be given a copy to take home. You are free to 
stop the research at any time without giving a reason. A decision to withdraw at any time, or a decision not to take part, will not affect the standard of care you receive.

\section{What will happen to me if I take part?}

At the start, the study will be explained to you by the study doctor. The surgeon looking after your routine care may telephone or during clinic ask your permission to receive a telephone call from Professor Stephens or his team to provide more information on the research. If you agree to take part we ask that you sign the consent form. Any treatments which you usually take will not change. Before your scheduled operation we will collect a blood sample from you. During the scheduled operation, the surgeon will remove a small piece of fat from inside the abdomen (about the size of a sugar cube) and underneath the skin. This should not cause any problems and will not have an effect on the planned operation. After the scheduled operation there will be no need for any further samples or action.

\section{Expenses and payments?}

There are no payments for taking part in this study.

\section{What do I have to do?}

If you agree to take part you will then be asked to complete the consent form, give a blood sample and then the surgeon will remove the samples of fat during the operation. Before the operation you should just follow the routine advice given. There is nothing else you need to do.

\section{What are the benefits of taking part?}

There is no direct benefit to you of taking part in the research but the information we get from this study might help improve the treatment of people with obesity and diabetes.

\section{What are the risks of taking part?}

There are no extra risks in taking part in this study as your routine medical care is not changed. There will be risks related to the main operation which your doctor will discuss with you beforehand. For the research, taking the small piece of fat does not affect the procedure being performed. The fat biopsy might cause a small amount of bleeding but this can be stopped immediately and the risk of internal scarring if extremely minimal.

\section{What happens when the study stops?}

No further samples will be necessary. We will dispose of all samples (fat and blood) after the study has completed.

\section{What if there is a problem?}

Participating in the study should not put you at any increased risk of anything going wrong. Part 2 of this sheet provides information on how to deal with any problems which arise.

\section{Will my taking part be kept confidential?}

Yes. We will follow ethical and legal practice and all information about you will be handled in confidence. The details are included in Part 2.

\section{If the information in Part 1 has interested you and you are considering participation, please read the additional information in Part 2 before making any decision.}




\section{Part 2}

What will happen if I don't want to carry on with the study?

Your participation in this study is completely voluntary and you may refuse to participate and are free to withdraw from the study at any time. Your decision to withdraw from the study will not affect the relationship between you and your study doctor, nor affect any future medical treatment provided for you. If you withdraw from the study, we will dispose of the samples and withdraw them from the research.

\section{What if there is a problem?}

Participating in the study should not put you at any increased risk of anything going wrong. If you have a concern about any aspect of this study, you should ask to speak to the researchers who will do their best to answer your questions (Telephone: 01792 704078). If you remain unhappy and wish to complain formally, you can do this through the ABMU Complaints Procedure (Website: http://www.wales.nhs.uk/sitesplus/863/page/39350; email: ABM.Complaints@wales.nhs.uk). If taking part in this research project harms you there are no special compensation arrangements. If you are harmed due to someone's negligence then you may have grounds for legal action but you may have to pay for it.

\section{Will my taking part in this study be kept confidential?}

Yes. Any information and samples which are collected during this research will be kept strictly confidential. You will be given a unique identifying number and any information about you which leaves the hospital will have your name and address removed so that you cannot be recognised. Some parts of your medical records and the data collected for the study may be looked at by authorised hospital staff to check that the study is being carried out correctly. All will have a duty of confidentiality to you as a research participant and we will do our best to meet this duty.

\section{Involvement of the General Practitioner/Family doctor (GP)}

If you agree to take part in this study, your study doctor will contact your GP to let him/her know if you so wish. You will be asked to consent for your GP to be informed of your participation on the consent form.

\section{What will happen to any samples I give and will genetic tests be done?}

We consider the fat sample you donate for the research to be a gift. This sample will be used to look how changes regulation within fat influences proteins and hormones. No genetic tests for disease risk will take place. Samples will be stored by the investigators in a locked freezer within a locked laboratory in Swansea University. The samples may be kept for the duration of the research. All samples will be anonymised.

\section{What will happen to the results of the research study?}

The results of the study may be published in a medical journal and might be presented to other doctors and scientists with an interest in diabetes. If reports or publications are generated from the study you will not be identified in these. The results will also be used to look at the possibility of a larger more focused study involving fat.

\section{Who is organising and funding the research?}

This work is being organised between Abertawe Bro Morgannwg University Health Board and Swansea University College of Medicine. The work is being supported by funds from the Welsh Government.

\section{Who has reviewed the study?}

The East of Scotland Research Ethics Committee REC 1 has examined the proposal and has raised no objections from the point of view of research ethics. It is a requirement that your records in this research, together with any relevant medical records, be made available for scrutiny by monitors 
from the Abertawe Bro Morgannwg University Health Board, whose role is to check that research is properly conducted and the interests of those taking part are adequately protected.

\section{Who can I contact for further information?}

This Patient Information Sheet and the Informed Consent Form contain important facts which you should consider when deciding whether you are willing to take part in this study. If at any time you have any questions about the study, your rights as a research participant, a study related injury or side effects you should contact either of the two research doctors below or the Director of Research and Development within the local Health Board:
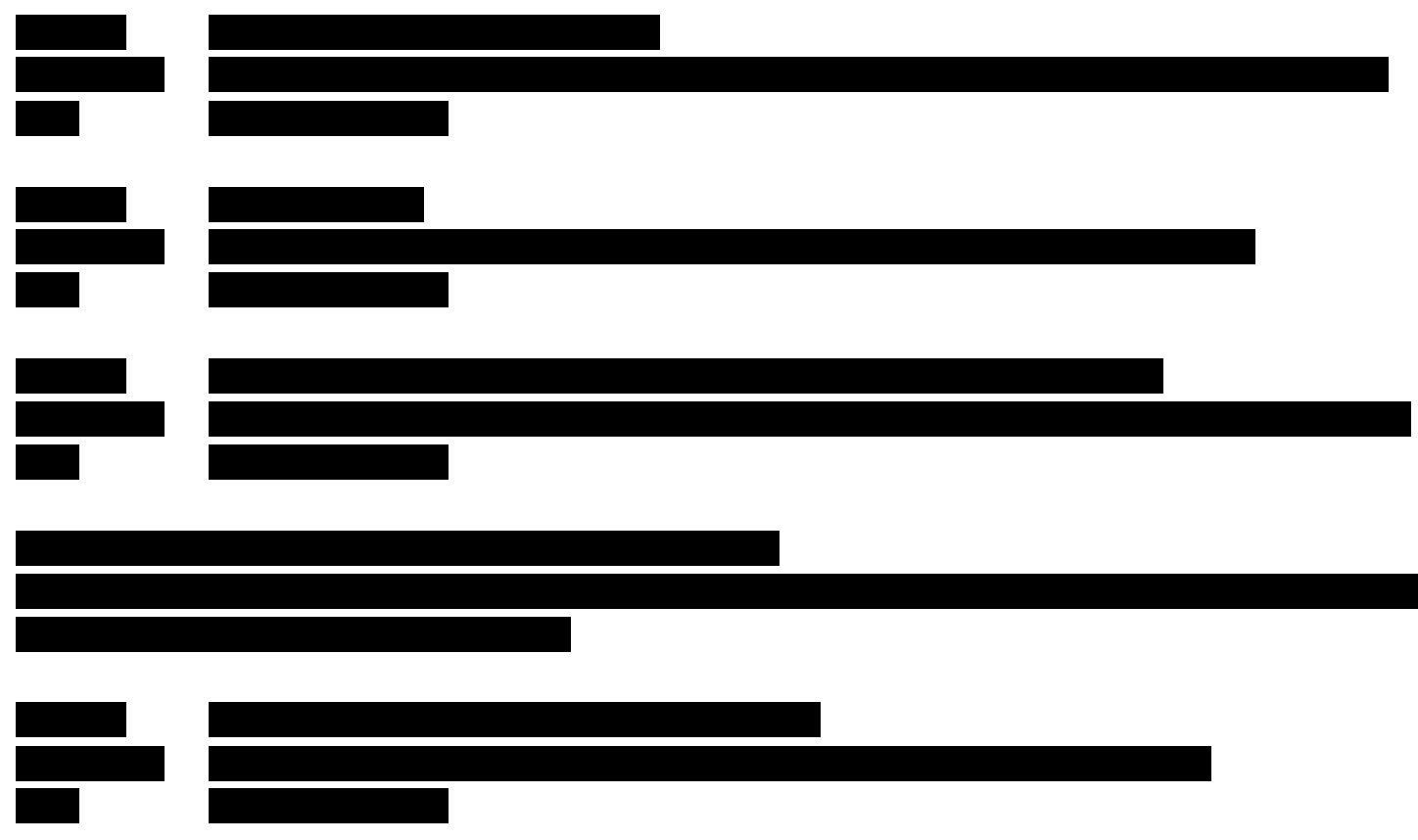

Thank you for reading this information sheet. 
Appendix 2

Primer sequences \& programmes 
Primer Sequences

\begin{tabular}{|c|c|c|c|}
\hline Primer & Forward Primer & Reverse Primer & Source \\
\hline B-actin & $\begin{array}{c}\text { GATGGCCACGGCTG } \\
\text { CTTC }\end{array}$ & $\begin{array}{c}\text { TGCCTCAGGGCAGC } \\
\text { GGAA }\end{array}$ & $\begin{array}{c}\text { Eurofins MWG } \\
\text { operon }\end{array}$ \\
\hline GHRL & $\begin{array}{c}\text { TGAGCCCTGAACAC } \\
\text { CAGAGAG }\end{array}$ & $\begin{array}{c}\text { AAAGCCAGATGAG } \\
\text { CGCTTCTA }\end{array}$ & $\begin{array}{c}\text { Eurofins MWG } \\
\text { operon }\end{array}$ \\
\hline PPARY & $\begin{array}{l}\text { ACAGCGACTTGGCA } \\
\text { ATATTTATTG }\end{array}$ & $\begin{array}{c}\text { AGCTCCAGGGCTTG } \\
\text { TAGCA }\end{array}$ & $\begin{array}{c}\text { Eurofins MWG } \\
\text { operon }\end{array}$ \\
\hline$A B C G 1$ & $\begin{array}{l}\text { TCCTATGTCAGGTA } \\
\text { TGGGTTCG }\end{array}$ & $\begin{array}{c}\text { GTCCAGGTACAGCT } \\
\text { TGGCAT }\end{array}$ & $\begin{array}{c}\text { Eurofins MWG } \\
\text { operon }\end{array}$ \\
\hline LXRB & $\begin{array}{c}\text { CCTGCAGGTGGAGT } \\
\text { TCATCA }\end{array}$ & $\begin{array}{c}\text { CAGCTGGTCCTGCG } \\
\text { GC }\end{array}$ & $\begin{array}{c}\text { Eurofins MWG } \\
\text { operon }\end{array}$ \\
\hline LYPLA1 & $\begin{array}{c}\text { GGTCCTATCGGTGG } \\
\text { TGCTAA }\end{array}$ & $\begin{array}{c}\text { ACATCCATCATTTCC } \\
\text { TGTTGACAC }\end{array}$ & $\begin{array}{c}\text { Eurofins MWG } \\
\text { operon }\end{array}$ \\
\hline SREBF1 & $\begin{array}{c}\text { CTTAGAGCGAGCAC } \\
\text { TGAACTG }\end{array}$ & $\begin{array}{c}\text { CCGAGGGCATCCGA } \\
\text { GAATT }\end{array}$ & PrimerDesign \\
\hline mBOAT4 & $\begin{array}{c}\text { TCTTTGTCTGAGCA } \\
\text { TGTGTGTAA }\end{array}$ & $\begin{array}{c}\text { AAGCACTGGACCCT } \\
\text { TGAACA }\end{array}$ & PrimerDesign \\
\hline
\end{tabular}

Table 1. Primer sequences generated through NCBI primer tools and PrimerDesign 


\section{Primer Cycling Conditions}

\begin{tabular}{|c|c|}
\hline Programme & Primer Sets \\
\hline $\begin{array}{l}95^{\circ} \mathrm{C} \text { for } 3 \mathrm{~min} \\
35 \text { cycles of } \\
95^{\circ} \mathrm{C} \text { for } 30 \mathrm{sec} \\
60^{\circ} \mathrm{C} \text { for } 30 \mathrm{sec} \\
72^{\circ} \mathrm{C} \text { for } 30 \mathrm{sec}\end{array}$ & $\begin{array}{l}\text { B-actin } \\
\text { LYPLA1 } \\
\text { PPARY }\end{array}$ \\
\hline $\begin{array}{l}95^{\circ} \mathrm{C} \text { for } 3 \mathrm{~min} \\
35 \mathrm{cycles} \text { of } \\
95^{\circ} \mathrm{C} \text { for } 30 \mathrm{sec} \\
59.5^{\circ} \mathrm{C} \text { for } 30 \mathrm{sec} \\
72^{\circ} \mathrm{C} \text { for } 30 \mathrm{sec}\end{array}$ & $\begin{array}{c}A B C G 1 \\
\text { LXRB }\end{array}$ \\
\hline $\begin{array}{l}95^{\circ} \mathrm{C} \text { for } 3 \mathrm{~min} \\
35 \mathrm{cycles} \text { of } \\
95^{\circ} \mathrm{C} \text { for } 30 \mathrm{sec} \\
58.5^{\circ} \mathrm{C} \text { for } 30 \mathrm{sec} \\
72^{\circ} \mathrm{C} \text { for } 30 \mathrm{sec}\end{array}$ & GHRL \\
\hline $\begin{array}{l}95^{\circ} \mathrm{C} \text { for } 2 \mathrm{~min} \\
40 \text { cycles of } \\
95^{\circ} \mathrm{C} \text { for } 15 \mathrm{sec} \\
60^{\circ} \mathrm{C} \text { for } 60 \mathrm{sec}\end{array}$ & $\begin{array}{l}\text { SREBF1 } \\
\text { mBOAT4 }\end{array}$ \\
\hline
\end{tabular}

Table 2. Cycling conditions with optimal annealing temperature in bold for primer sequences. 
Appendix 3

Primer optimisation \& validation 


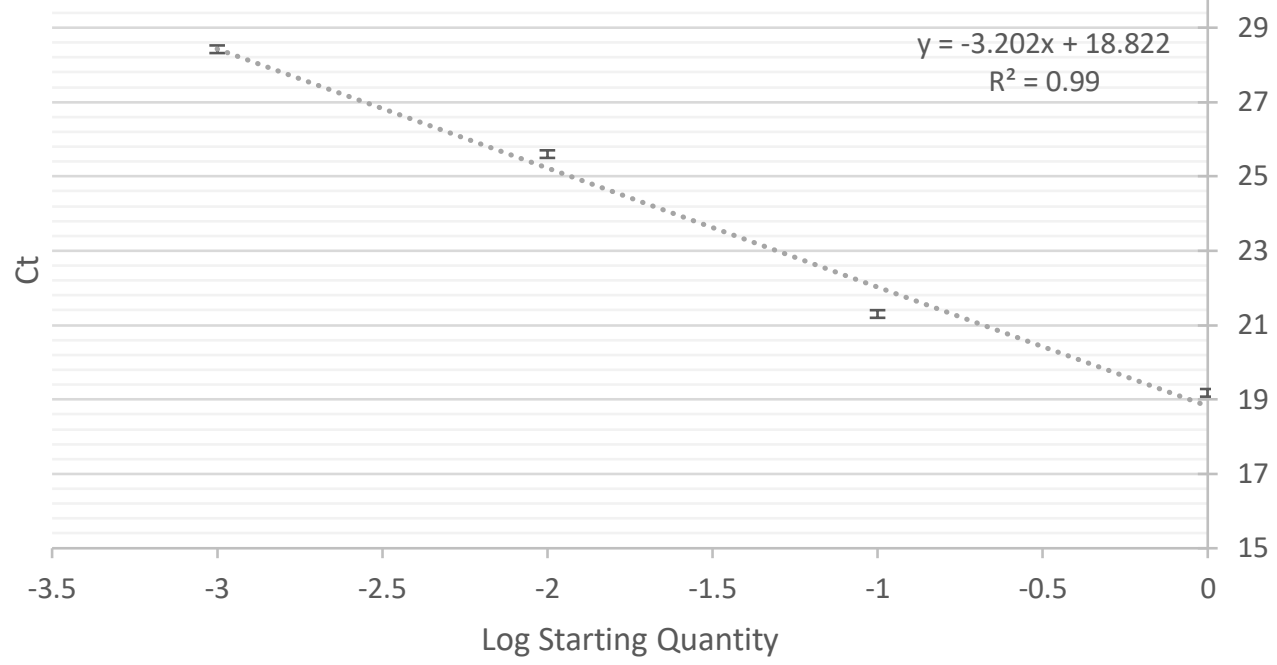

Figure 1. $\beta$ actin standard curve 10 -fold dilution over 4 points. Log starting quantity vs cycle threshold $(C t) \cdot y=-3.202 x+18.822, R^{2}=0.99$. Efficiency $=105 \%$. Error bars indicate standard deviation. 


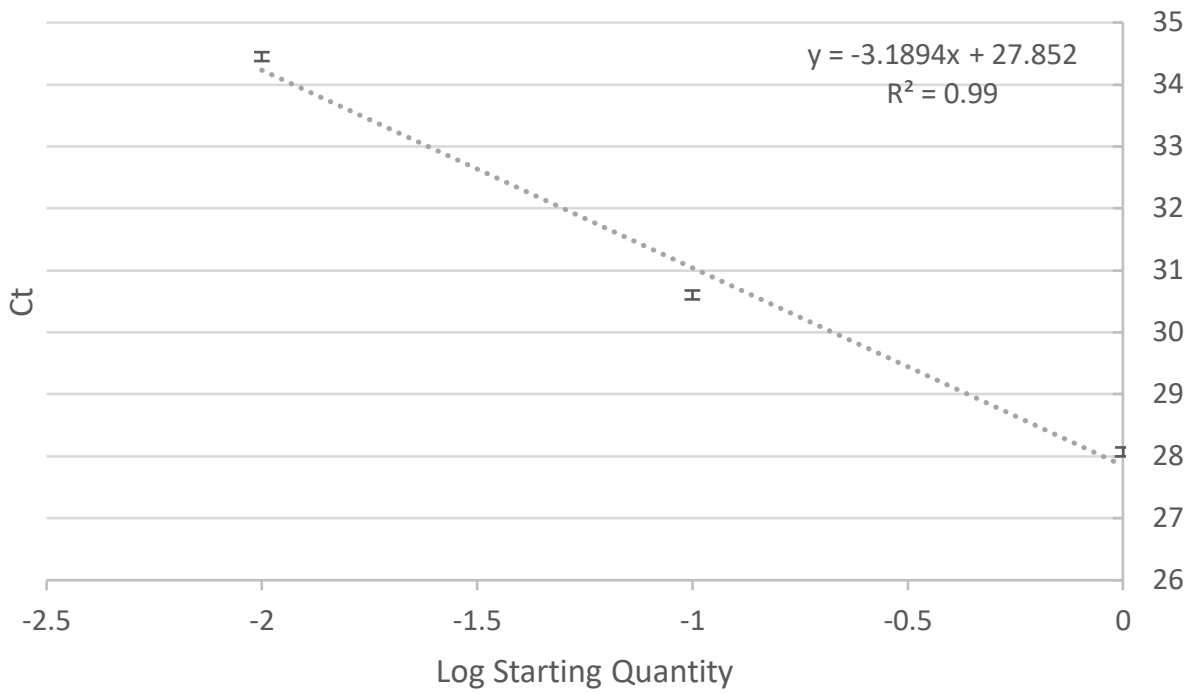

Figure 2. Ghrelin standard curve 10 -fold dilution over 3 points. Log starting quantity vs cycle threshold (Ct). $y=-3.1894 x+27.852, R^{2}=0.99$. Efficiency $=106 \%$. Error bars indicate standard deviation.

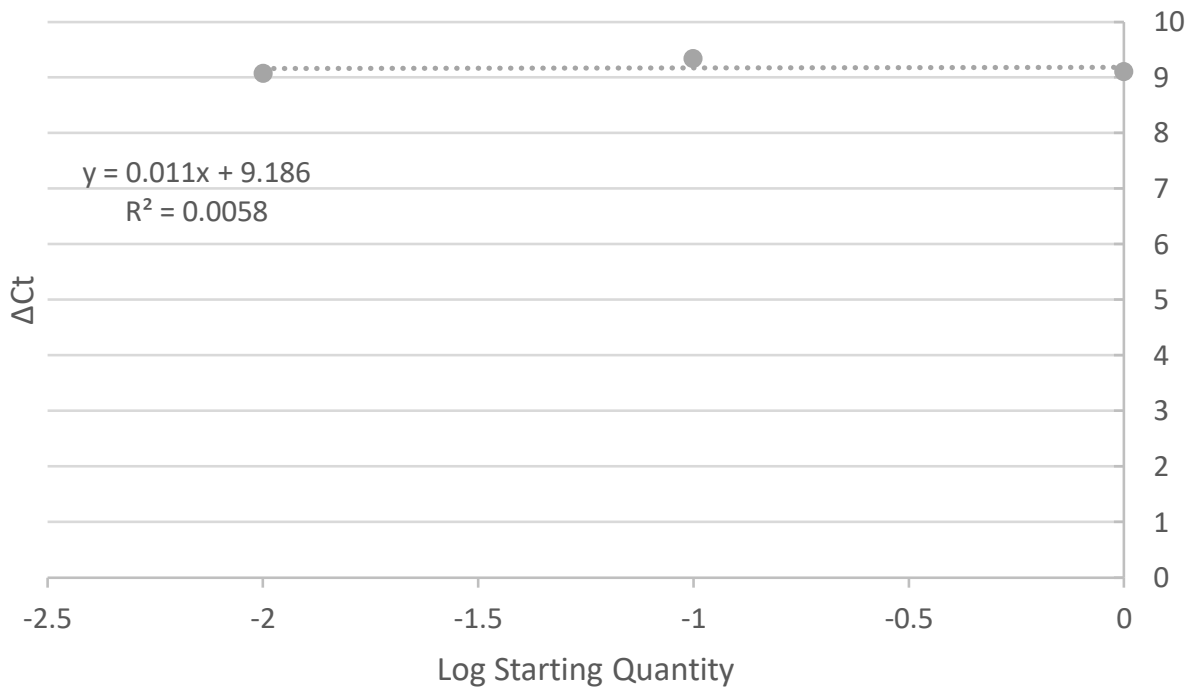

Figure 3. Ghrelin primer set validation semi-regression plot over 3 points. Log starting quantity vs delta cycle threshold $(\Delta \mathrm{Ct}) \cdot \mathrm{y}=0.011 \mathrm{x}+9.186, \mathrm{R}^{2}=0.0058$ 


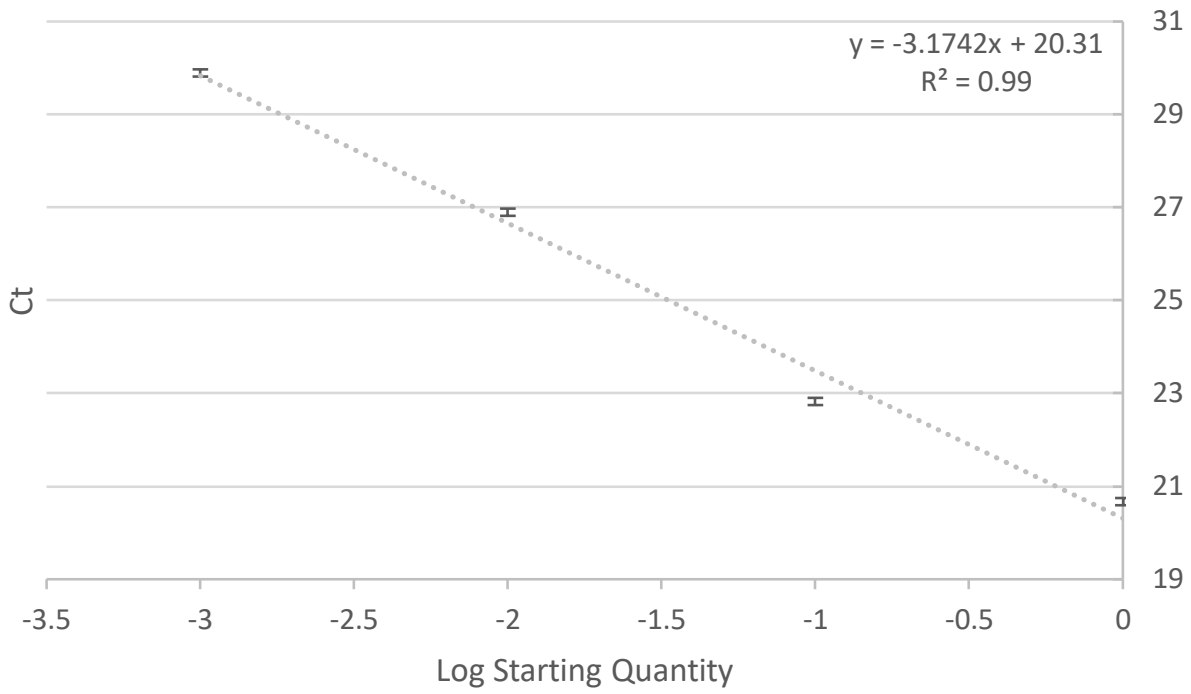

Figure 4. PPARy standard curve 10 -fold dilution over 4 points. Log starting quantity vs cycle threshold $(C t) \cdot y=-3.1742 x+20.31, R^{2}=0.99$. Efficiency $=107 \%$. Error bars indicate standard deviation.

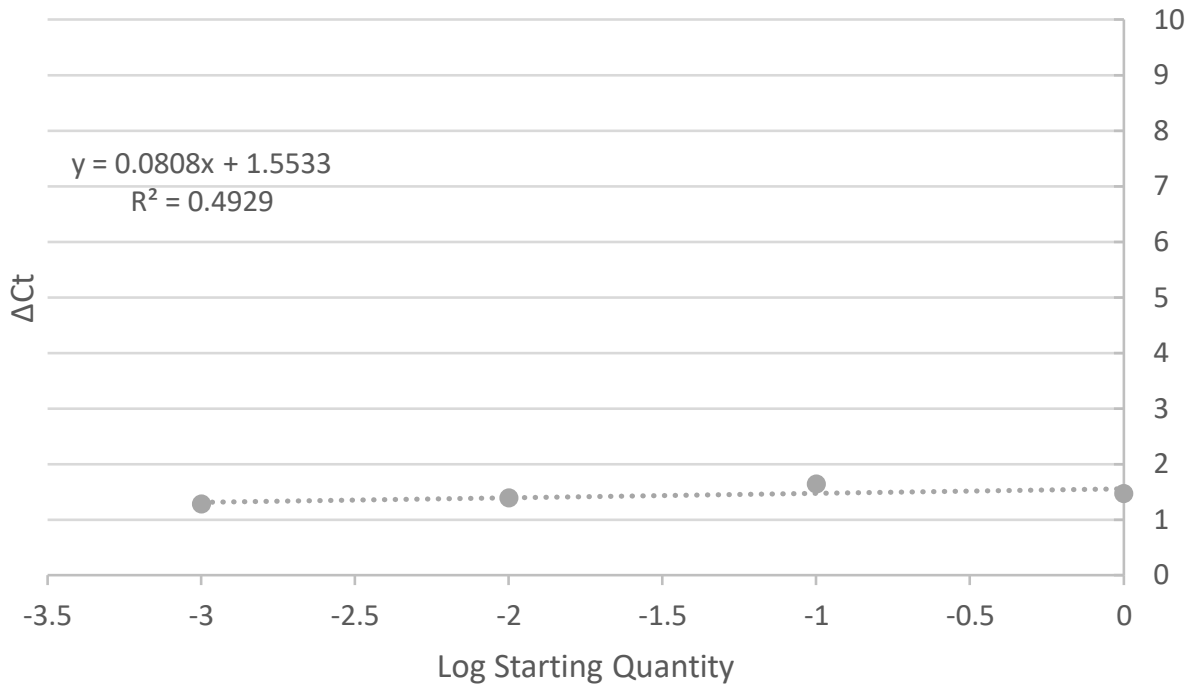

Figure 5. PPARy primer set validation semi-regression plot over 4 points. Log starting quantity vs delta cycle threshold $(\Delta \mathrm{Ct}) \cdot \mathrm{y}=0.0808 \mathrm{x}+1.5533, \mathrm{R}^{2}=0.4929$ 


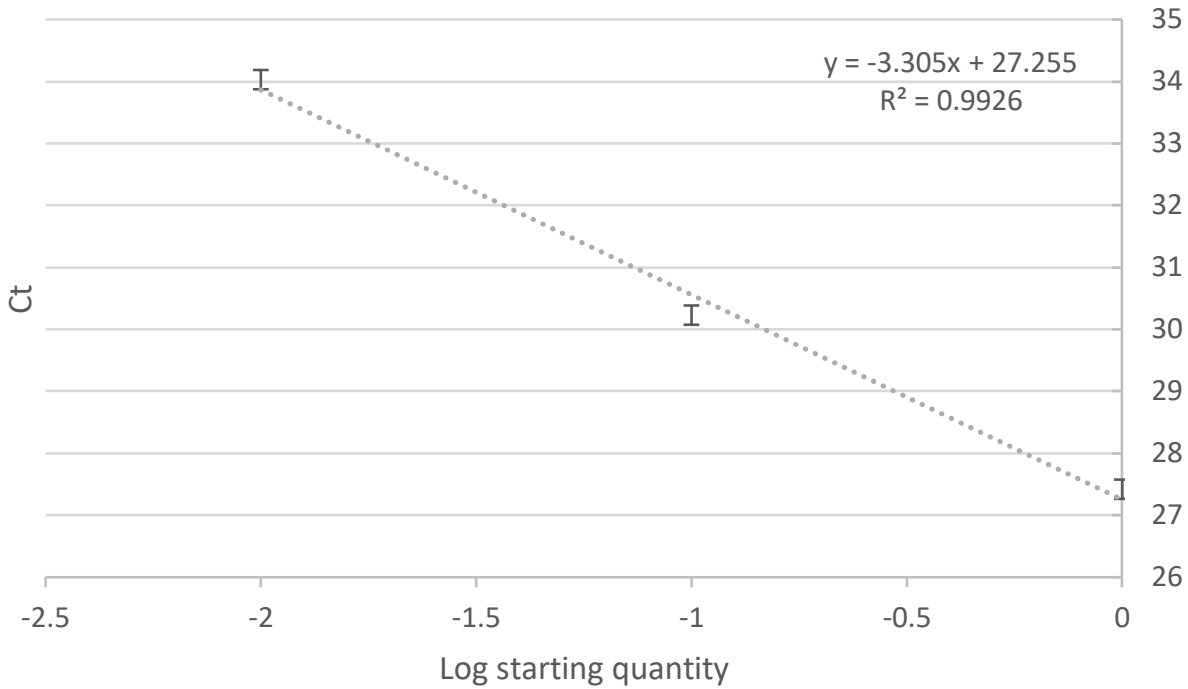

Figure 6. ABCG1 standard curve 10-fold dilution over 3 points. Log starting quantity vs cycle threshold (Ct). $y=-3.305 x+27.255, R^{2}=0.9926$. Efficiency $=101 \%$. Error bars indicate standard deviation.

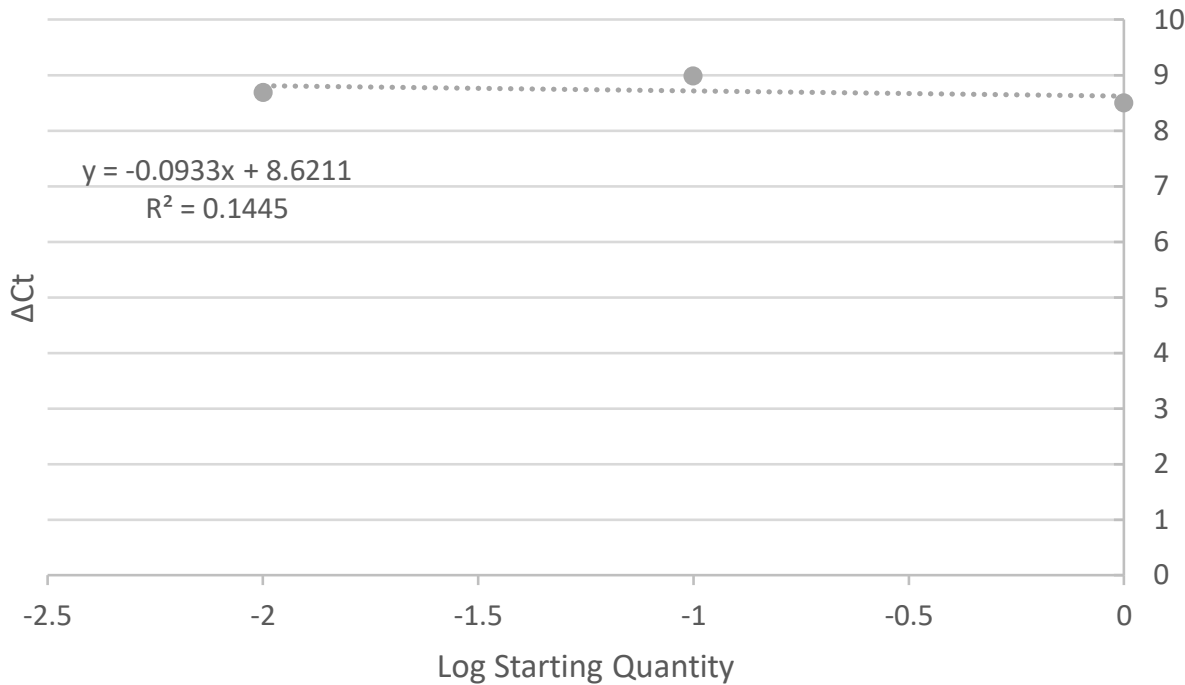

Figure 7. ABCG1 primer set validation semi-regression plot over 3 points. Log starting quantity vs delta cycle threshold $(\Delta \mathrm{Ct}) \cdot \mathrm{y}=-0.0933 x+8.6211, \mathrm{R}^{2}=0.1445$ 


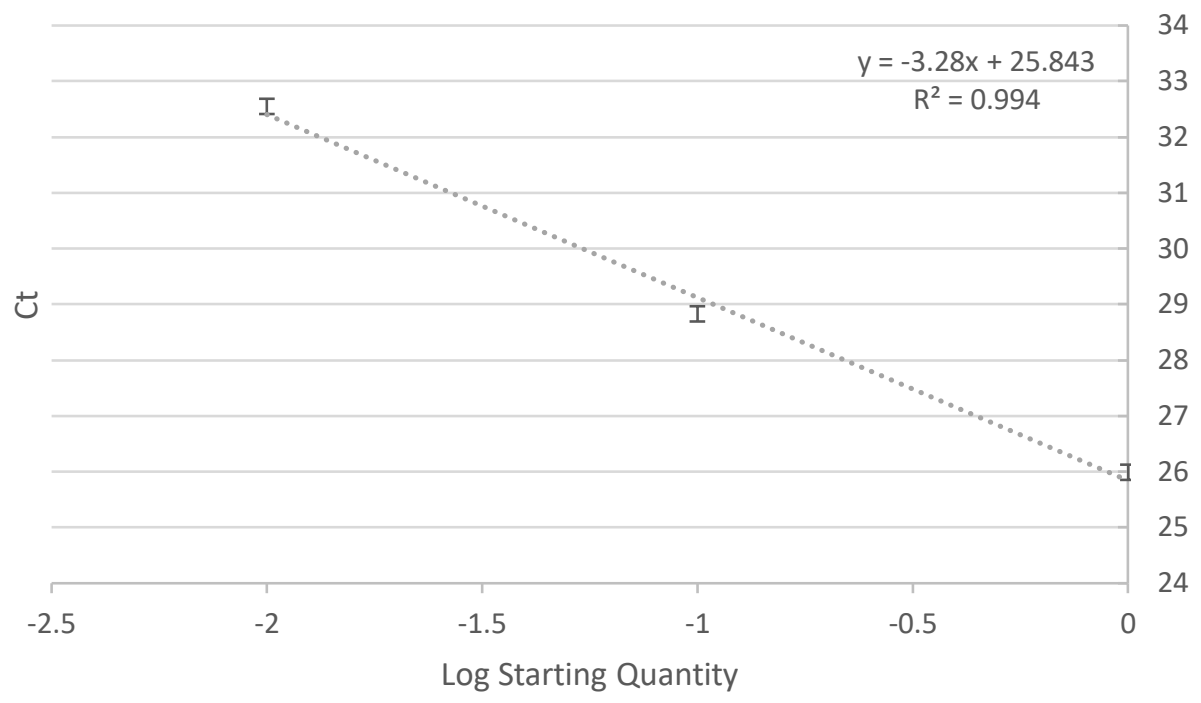

Figure 8. LXR $\beta$ standard curve 10 -fold dilution over 3 points. Log starting quantity vs cycle threshold $(C t) . y=-3.28 x+25.843, R^{2}=0.994$. Efficiency $=102 \%$. Error bars indicate standard deviation.

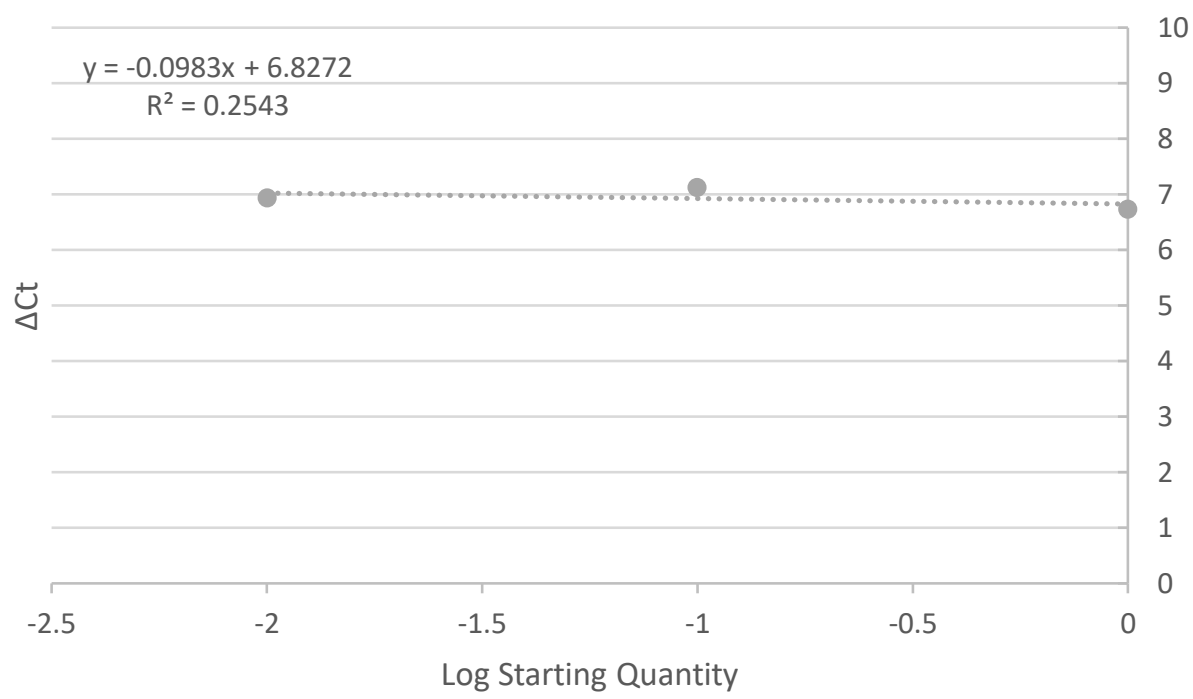

Figure 9. LXR $\beta$ primer set validation semi-regression plot over 3 points. Log starting quantity vs delta cycle threshold $(\Delta C t) \cdot y=-0.0983 x+6.8272, R^{2}=0.2543$ 


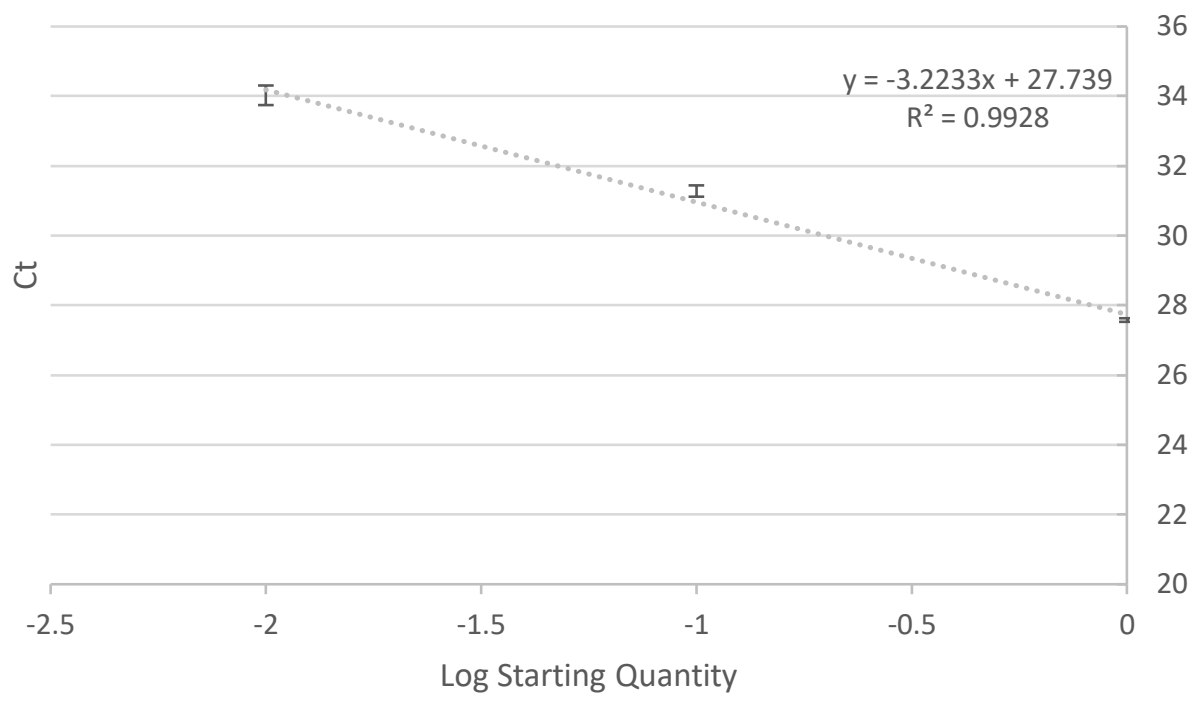

Figure 10. LXR $\beta$ standard curve 10-fold dilution over 3 points. Log starting quantity vs cycle threshold $(C t) \cdot y=-3.2233 x+27.739, R^{2}=0.9928$. Efficiency $=104 \%$. Error bars indicate standard deviation

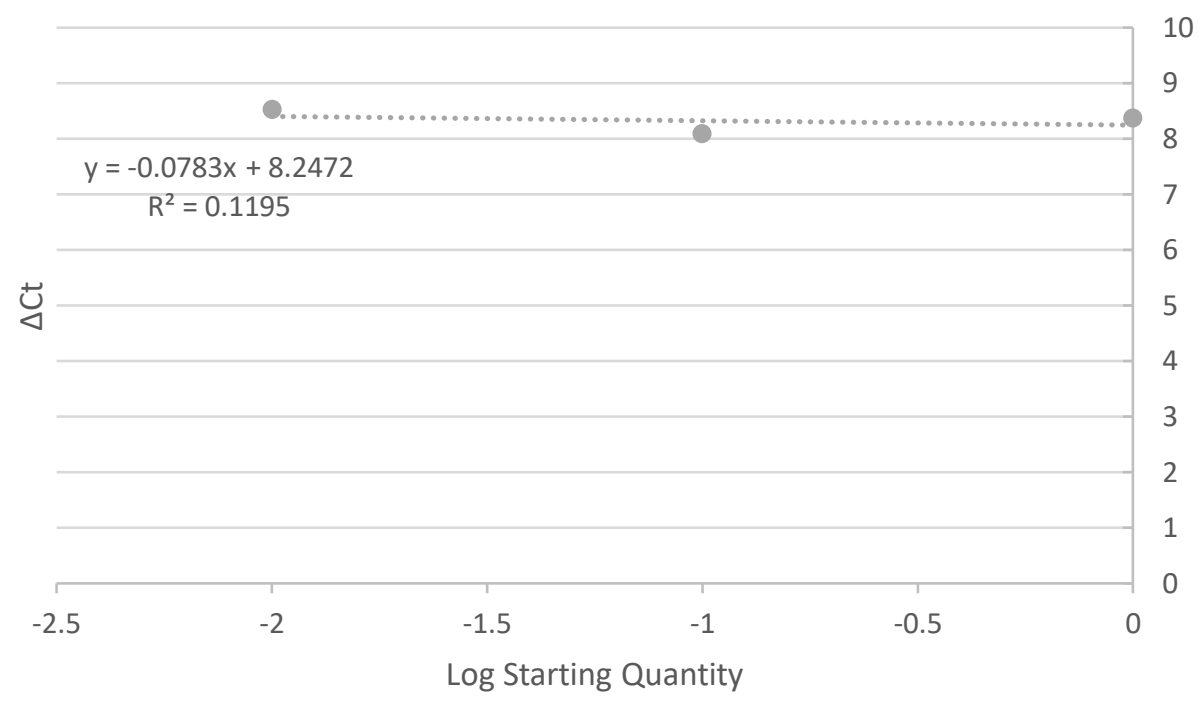

Figure 11. LYPLA1 primer set validation semi-regression plot over 3 points. Log starting quantity vs delta cycle threshold $(\Delta \mathrm{Ct}) \cdot \mathrm{y}=-0.0783 \mathrm{x}+8.2472, \mathrm{R}^{2}=0.1195$ 


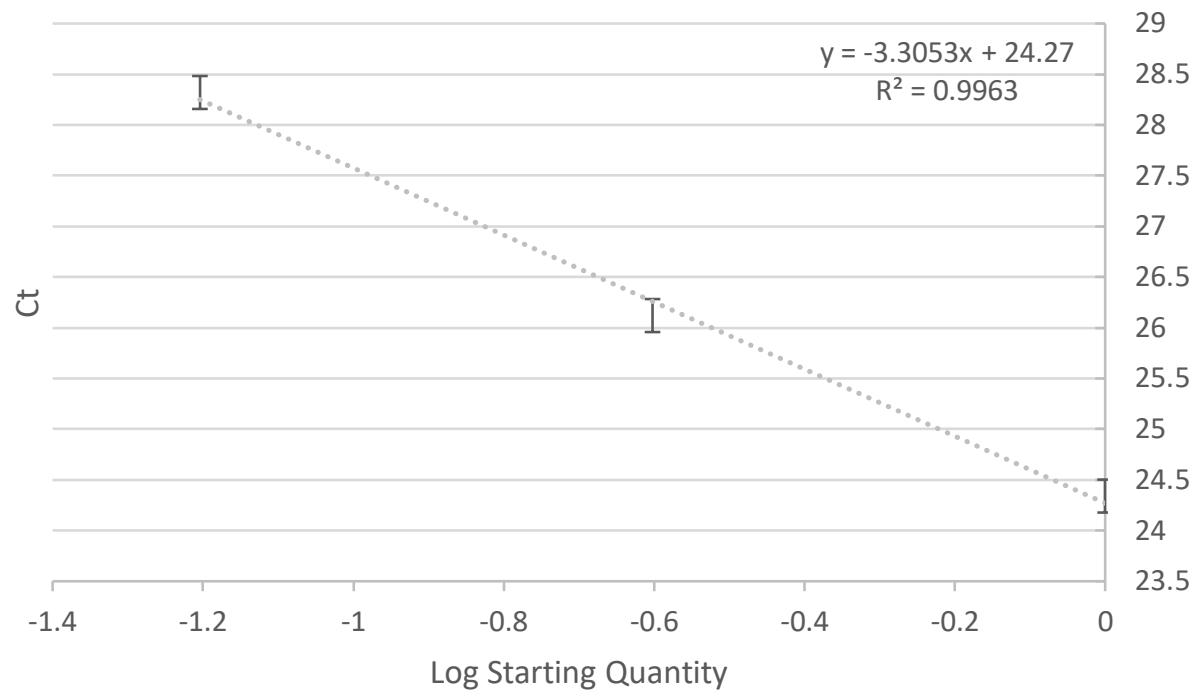

Figure 12. SREBF1 standard curve 1 in 4 dilution series over 3 points. Log starting quantity vs cycle threshold (Ct). $y=-3.3053 x+24.27, R^{2}=0.9963$. Efficiency $=101 \%$. Error bars indicate standard deviation.

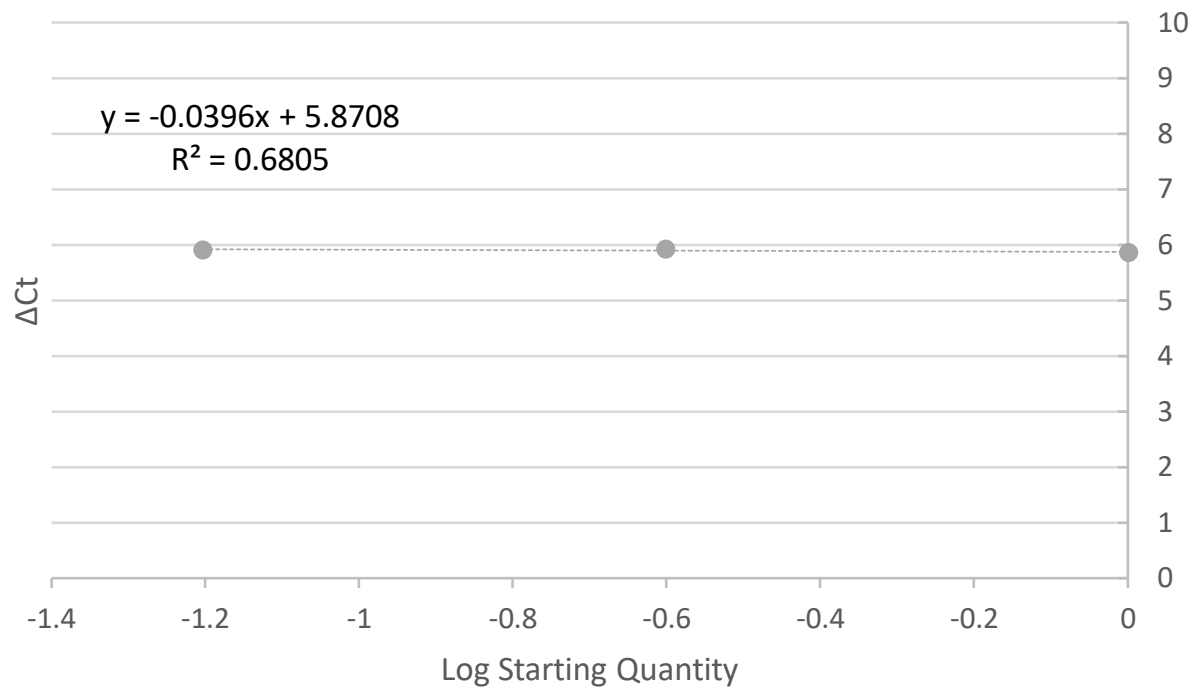

Figure 13. SREBF1 primer set validation semi-regression plot over 3 points. Log starting quantity vs delta cycle threshold $(\Delta \mathrm{Ct}) \cdot \mathrm{y}=-0.0396 \mathrm{x}+5.8708, \mathrm{R}^{2}=0.6805$ 


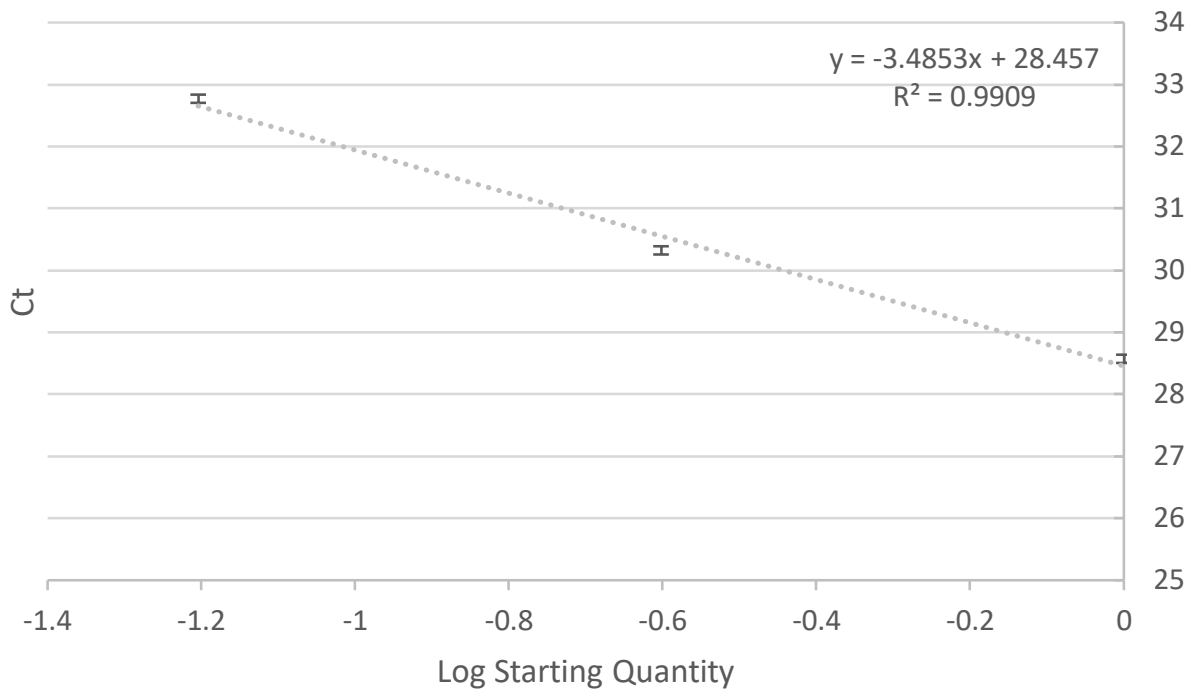

Figure 14. mBOAT4 standard curve 1 in 4 dilution series over 3 points. Log starting quantity vs cycle threshold $(C t) . y=-3.4853 x+28.457, R^{2}=0.99$. Efficiency $=94 \%$. Error bars indicate standard deviation.

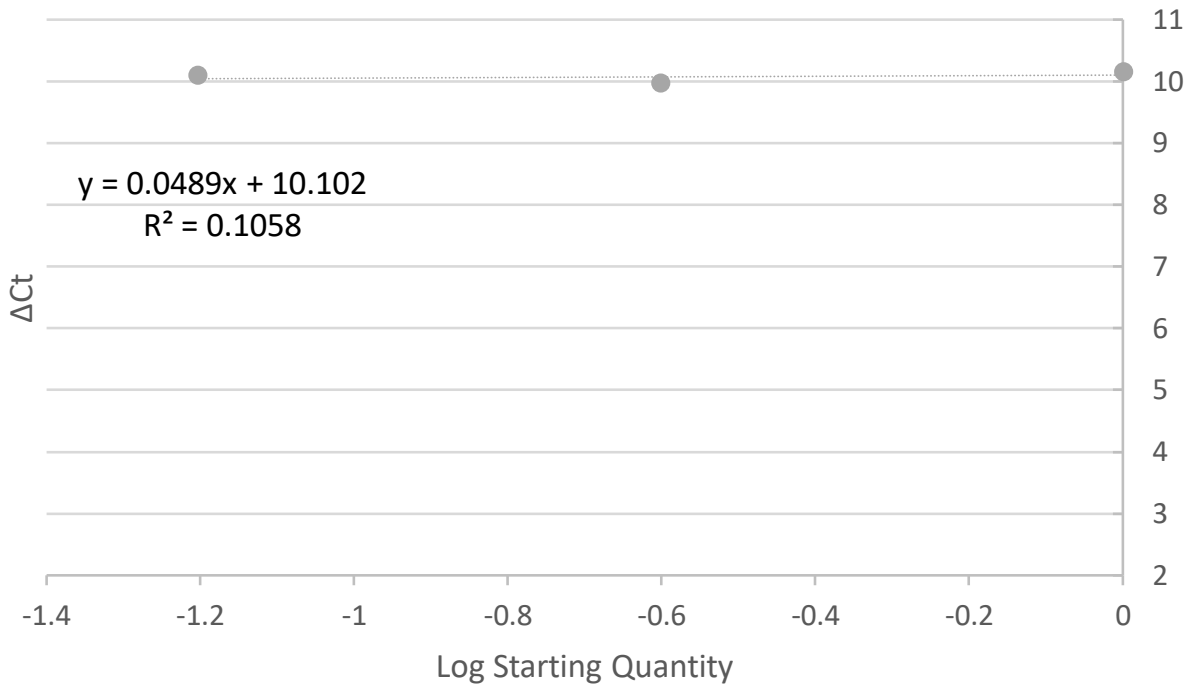

Figure 15. mBOAT4 primer set validation semi-regression plot over 3 points. Log starting quantity vs delta cycle threshold $(\Delta \mathrm{Ct}) \cdot \mathrm{y}=0.0489 \mathrm{x}+10.102, \mathrm{R}^{2}=0.1058$ 


\section{Appendix 4 \\ Real Time PCR validation \\ (Raw data)}




\begin{tabular}{|c|c|c|c|c|c|c|c|}
\hline Primer Set & Replicate & \multicolumn{3}{|c|}{$\begin{array}{l}\text { Intra assay } \\
\text { Variation }\end{array}$} & \multicolumn{3}{|c|}{$\begin{array}{l}\text { Inter assay } \\
\text { variation }\end{array}$} \\
\hline \multirow{15}{*}{ PPARY } & \multirow[t]{3}{*}{1} & 22.0 & 25.5 & 19.2 & 22.0 & 25.5 & 19.2 \\
\hline & & 22.0 & 24.4 & 19.2 & 22.0 & 24.4 & 19.2 \\
\hline & & 22.2 & 24.8 & 19.1 & 22.2 & 24.8 & 19.1 \\
\hline & \multirow[t]{3}{*}{2} & 22.1 & 24.9 & NA & 23.0 & 24.0 & 18.9 \\
\hline & & 22.1 & 24.3 & 19.7 & 23.1 & 24.4 & 18.8 \\
\hline & & 22.0 & 24.3 & 19.9 & 22.9 & 24.2 & 18.9 \\
\hline & \multirow[t]{3}{*}{3} & 22.2 & 25.2 & 19.9 & 22.5 & 25.1 & 19.5 \\
\hline & & 22.2 & 25.2 & 19.9 & 22.7 & 25.4 & 19.7 \\
\hline & & 21.8 & 25.6 & 19.5 & 22.9 & 25.0 & 19.2 \\
\hline & \multirow[t]{3}{*}{4} & 21.8 & 24.9 & 20.1 & 23.1 & 24.8 & 19.6 \\
\hline & & 21.8 & 24.9 & 20.1 & 22.8 & 24.6 & 19.9 \\
\hline & & 21.6 & 24.3 & 19.8 & 22.4 & 24.6 & 20.2 \\
\hline & \multirow[t]{3}{*}{5} & 22.4 & 25.3 & 19.7 & 22.6 & 24.1 & 19.3 \\
\hline & & 22.2 & 25.3 & 19.7 & 22.6 & 24.2 & 19.3 \\
\hline & & 22.5 & 25.5 & 19.9 & 22.7 & 23.9 & 19.4 \\
\hline \multirow{15}{*}{ GHRL } & \multirow[t]{3}{*}{1} & 27.6 & 29.8 & 31.5 & 27.6 & 29.8 & 31.5 \\
\hline & & 27.7 & 30.2 & 32.0 & 27.7 & 30.2 & 32.0 \\
\hline & & 27.6 & 30.4 & 32.0 & 27.6 & 30.4 & 32.0 \\
\hline & \multirow[t]{3}{*}{2} & 27.6 & 29.9 & 32.2 & 27.6 & 29.6 & 32.1 \\
\hline & & 27.6 & 30.0 & 32.4 & 28.0 & 30.1 & 32.9 \\
\hline & & 27.7 & 30.0 & 32.2 & 27.2 & 30.0 & 32.1 \\
\hline & \multirow[t]{3}{*}{3} & 28.0 & 29.7 & 31.8 & 27.7 & 29.7 & 31.4 \\
\hline & & 28.1 & 29.8 & 31.1 & 27.7 & 29.7 & 31.5 \\
\hline & & 27.2 & 29.8 & 30.9 & 28.1 & 30.1 & 31.3 \\
\hline & \multirow[t]{3}{*}{4} & 28.2 & 29.3 & 31.5 & 27.5 & 28.9 & 31.9 \\
\hline & & 28.5 & 29.9 & 32.0 & 27.3 & 29.3 & 32.3 \\
\hline & & 27.9 & 30.0 & 32.0 & 27.5 & 29.4 & 32.4 \\
\hline & \multirow[t]{3}{*}{5} & 27.2 & 30.3 & 32.4 & 28.1 & 29.9 & 32.9 \\
\hline & & 27.4 & 29.9 & 32.1 & 28.5 & 30.1 & 32.6 \\
\hline & & 27.6 & 29.8 & 32.0 & 27.9 & 30.2 & 32.6 \\
\hline \multirow{15}{*}{ mBOAT4 } & \multirow[t]{3}{*}{1} & 21.7 & 30.2 & 27.8 & 21.7 & 30.2 & 27.8 \\
\hline & & 22.0 & 29.8 & 27.5 & 22.0 & 29.8 & 27.5 \\
\hline & & 21.2 & 30.2 & 27.8 & 21.2 & 30.2 & 27.8 \\
\hline & \multirow[t]{3}{*}{2} & 21.6 & 29.6 & 27.5 & 22.1 & 30.5 & 27.8 \\
\hline & & 21.7 & 29.7 & 27.8 & 22.3 & 30.6 & 27.9 \\
\hline & & 21.4 & 29.4 & 27.8 & 22.3 & 30.5 & 28.0 \\
\hline & \multirow[t]{3}{*}{3} & 21.7 & 29.4 & 27.1 & 21.5 & 29.6 & 28.1 \\
\hline & & 21.7 & 29.5 & 27.0 & 21.5 & 29.9 & 27.9 \\
\hline & & 21.8 & 29.4 & 28.0 & 21.6 & 30.3 & 27.9 \\
\hline & \multirow[t]{3}{*}{4} & 21.7 & 30.0 & 27.4 & 22.4 & 30.9 & 27.1 \\
\hline & & 21.7 & 30.1 & 27.6 & 22.3 & 31.0 & 28.0 \\
\hline & & 22.0 & 29.8 & 27.8 & 22.6 & 31.3 & 27.4 \\
\hline & \multirow[t]{3}{*}{5} & 21.8 & 29.7 & 27.1 & 21.2 & 29.7 & 27.6 \\
\hline & & 21.8 & 29.7 & 27.7 & 21.8 & 29.8 & 28.6 \\
\hline & & 21.8 & 30.2 & 27.6 & 21.9 & 29.8 & 28.4 \\
\hline
\end{tabular}




\begin{tabular}{|c|c|c|c|c|c|c|c|}
\hline Primer Set & Replicate & \multicolumn{3}{|c|}{$\begin{array}{l}\text { Intra assay } \\
\text { Variation }\end{array}$} & \multicolumn{3}{|c|}{$\begin{array}{c}\text { Inter assay } \\
\text { variation }\end{array}$} \\
\hline \multirow{15}{*}{$A B C G 1$} & \multirow[t]{3}{*}{1} & 27.2 & 26.7 & 23.7 & 27.2 & 26.7 & 23.7 \\
\hline & & 27.3 & 26.7 & 23.7 & 27.3 & 26.7 & 23.7 \\
\hline & & 27.2 & 26.6 & 23.9 & 27.2 & 26.6 & 23.9 \\
\hline & \multirow[t]{3}{*}{2} & 27.2 & 26.3 & 23.9 & 27.7 & 26.1 & 24.1 \\
\hline & & 27.2 & 26.4 & 23.9 & 27.8 & 26.1 & 24.3 \\
\hline & & 27.7 & 26.8 & 23.8 & 27.7 & 26.2 & 24.4 \\
\hline & \multirow[t]{3}{*}{3} & 27.2 & 26.6 & 24.4 & 27.4 & 26.7 & 23.6 \\
\hline & & 27.5 & 26.7 & 23.7 & 27.5 & 26.7 & 24.5 \\
\hline & & 27.5 & 26.7 & 24.5 & 27.3 & 26.9 & 24.1 \\
\hline & \multirow[t]{3}{*}{4} & 27.8 & 26.4 & 23.9 & 26.9 & 26.2 & 23.8 \\
\hline & & 27.7 & 26.7 & 24 & 27 & 26.3 & 24.3 \\
\hline & & 27.4 & 26.3 & 23.7 & 26.9 & 26.6 & 24.2 \\
\hline & \multirow[t]{3}{*}{5} & 27.4 & 27.1 & 23.8 & 27 & 26.9 & 24.3 \\
\hline & & 27.3 & 26.8 & 23.6 & 27.2 & 26.9 & 24.4 \\
\hline & & 27.4 & 26.8 & 24.2 & 27.3 & 26.7 & 23.8 \\
\hline \multirow{15}{*}{ LXRB } & \multirow[t]{3}{*}{1} & 23.2 & 24.9 & 28.7 & 23.2 & 24.9 & 28.7 \\
\hline & & 23.2 & 24.6 & 29.1 & 23.2 & 24.6 & 29.1 \\
\hline & & 23 & 24.6 & 28.7 & 23 & 24.6 & 28.7 \\
\hline & \multirow[t]{3}{*}{2} & 23.3 & 24.6 & 28.9 & 23.8 & 25 & 29.7 \\
\hline & & 23.1 & 24.9 & 29.1 & 23.9 & 24.9 & 30.1 \\
\hline & & 22.9 & 25 & 28.8 & 23.9 & 24.6 & 29.5 \\
\hline & \multirow[t]{3}{*}{3} & 23.1 & 24.3 & 28.9 & 23.7 & 24.9 & 29.8 \\
\hline & & 23.3 & 25.1 & 28.9 & 23.4 & 24.6 & 29.5 \\
\hline & & 23.5 & 24.5 & 28.7 & 23.3 & 24.6 & 29.4 \\
\hline & \multirow[t]{3}{*}{4} & 23.7 & 25.1 & 28.5 & 23 & 23.2 & 28.7 \\
\hline & & 23.6 & 24.8 & 28.5 & 22.7 & 23.2 & 29.1 \\
\hline & & 23.9 & 24.6 & 29.1 & 22.4 & 23.9 & 29.2 \\
\hline & \multirow[t]{3}{*}{5} & 22.8 & 24.7 & 29 & NA & 24.3 & 30.4 \\
\hline & & 22.9 & 24.7 & 28.4 & 22.9 & 24.3 & 29.7 \\
\hline & & 23 & 24.5 & 28.5 & 22.9 & 24.9 & 29.9 \\
\hline \multirow{15}{*}{ SREBF1 } & \multirow[t]{3}{*}{1} & 23.6 & 21.3 & 23.5 & 23.6 & 21.3 & 23.5 \\
\hline & & 23.3 & 21.4 & 23.8 & 23.3 & 21.4 & 23.8 \\
\hline & & 23.2 & 21.3 & 23.6 & 23.2 & 21.3 & 23.6 \\
\hline & \multirow[t]{3}{*}{2} & 23.5 & 21.9 & 23.8 & 24.2 & 20.9 & 24.0 \\
\hline & & 23.4 & 21.2 & 23.8 & 24.1 & 20.9 & 24.0 \\
\hline & & 23.4 & 21.4 & 23.8 & NA & 21.0 & 23.9 \\
\hline & \multirow[t]{3}{*}{3} & 23.6 & 21.8 & 23.9 & 23.2 & 21.0 & 23.8 \\
\hline & & 24.0 & 20.8 & 23.7 & 23.4 & 21.2 & 23.8 \\
\hline & & 23.8 & 21.3 & 24.0 & 23.4 & 21.2 & 23.7 \\
\hline & \multirow[t]{3}{*}{4} & 23.5 & 21.5 & 23.1 & 23.8 & 21.6 & 23.9 \\
\hline & & 23.2 & 21.2 & 23.7 & 23.8 & 21.6 & 23.8 \\
\hline & & 23.7 & 21.3 & 23.7 & 23.9 & 21.4 & 23.9 \\
\hline & \multirow[t]{3}{*}{5} & 23.3 & 21.1 & 23.7 & 24.0 & NA & 23.7 \\
\hline & & 23.8 & 21.1 & 23.6 & 23.4 & 20.9 & 23.4 \\
\hline & & 23.3 & 21.3 & 24.2 & 23.5 & 20.9 & 23.4 \\
\hline
\end{tabular}




\begin{tabular}{|c|c|c|c|c|c|c|c|}
\hline Primer Set & Replicate & \multicolumn{3}{|c|}{$\begin{array}{l}\text { Intra assay } \\
\text { Variation }\end{array}$} & \multicolumn{3}{|c|}{$\begin{array}{c}\text { Inter assay } \\
\text { variation }\end{array}$} \\
\hline \multirow{15}{*}{ LYPLA1 } & \multirow[t]{3}{*}{1} & 22.5 & 25.5 & 21.4 & 22.5 & 25.5 & 21.4 \\
\hline & & 22.5 & 25.4 & 21.2 & 22.5 & 25.4 & 21.2 \\
\hline & & 22.5 & 25.3 & 21.2 & 22.5 & 25.3 & 21.2 \\
\hline & \multirow[t]{3}{*}{2} & 22.6 & 25.3 & 21.1 & 22.1 & 24.9 & 21.0 \\
\hline & & 22.6 & 25.5 & 21.1 & 22.1 & 25.0 & 21.0 \\
\hline & & 22.5 & 24.9 & 21.2 & 22.2 & 25.0 & 21.1 \\
\hline & \multirow[t]{3}{*}{3} & 22.4 & 25.3 & 21.5 & 22.3 & 25.4 & 21.2 \\
\hline & & 22.5 & 25.6 & 21.4 & 22.3 & 25.4 & 21.2 \\
\hline & & 22.4 & 25.6 & 21.3 & 22.5 & 25.3 & 21.3 \\
\hline & \multirow[t]{3}{*}{4} & 22.7 & 25.3 & 21.4 & 21.9 & 25.6 & 21.5 \\
\hline & & 22.5 & 25.1 & 21.3 & 21.9 & 25.5 & 21.4 \\
\hline & & 22.7 & 25.1 & 21.2 & 22.0 & 25.5 & 21.4 \\
\hline & \multirow[t]{3}{*}{5} & 22.6 & 25.6 & 21.5 & 22.5 & 25.5 & 21.0 \\
\hline & & 22.5 & 25.4 & 21.4 & 22.4 & 25.4 & 21.2 \\
\hline & & 22.4 & 25.6 & 21.3 & 22.4 & 25.4 & 21.2 \\
\hline \multirow{15}{*}{ B-actin } & \multirow[t]{3}{*}{1} & 20.3 & 19.1 & 15.4 & 20.3 & 19.1 & 15.4 \\
\hline & & 20.2 & 18.7 & 15.5 & 20.2 & 18.7 & 15.5 \\
\hline & & 20.3 & 18.7 & 15.4 & 20.3 & 18.7 & 15.4 \\
\hline & \multirow[t]{3}{*}{2} & 20.4 & 18.9 & 15.5 & 20.0 & 19.0 & 15.1 \\
\hline & & 20.6 & 18.9 & 15.5 & 19.9 & 19.1 & 15.0 \\
\hline & & 20.6 & 19.2 & 15.4 & 20.0 & 19.0 & 14.9 \\
\hline & \multirow[t]{3}{*}{3} & 20.3 & 19.2 & 15.3 & 20.4 & 18.8 & 15.4 \\
\hline & & 20.3 & 19.2 & 15.4 & 20.3 & 18.6 & 15.6 \\
\hline & & 20.2 & 19.1 & 15.5 & 20.2 & 18.6 & 15.6 \\
\hline & \multirow[t]{3}{*}{4} & 20.2 & 18.9 & 15.2 & 20.5 & 18.2 & 15.4 \\
\hline & & 20.4 & 18.9 & 15.6 & 20.2 & 18.5 & 15.2 \\
\hline & & 20.4 & 19.0 & 15.5 & 20.0 & 18.7 & 15.6 \\
\hline & \multirow[t]{3}{*}{5} & 20.7 & 19.0 & 15.3 & 19.8 & 19.1 & 15.1 \\
\hline & & 20.5 & 18.9 & 15.4 & 19.8 & 19.1 & 15.5 \\
\hline & & 20.4 & 19.0 & 15.4 & 20.2 & 19.0 & 15.3 \\
\hline
\end{tabular}

Table to represent intra- and inter- variation of all primer sets as discussed in Chapter 2 section 2.3.5. 


\section{Appendix 5}

\section{Raw $\Delta \Delta C T$ data for SGBS cells}

Data used in Chapter 3 


\section{B-actin referred to as Act throughout}

\section{$\Delta \Delta C t$ Values for $L X R \beta$}

\section{Hyperglycaemic environment at 17 hours}

\section{Vehicle v Acyl-ghrelin (AG)}

\section{Round 1}

$\Delta \mathrm{Ct}$ (Vehicle) $=$ Average LXR Ct- Average Act Ct $\quad \Delta \mathrm{Ct}(\mathrm{AG})=$ Average LXR Ct- AverageActCt

$\Delta$ Ct $($ Vehicle $)=25.68-20.39=5.28$

$\Delta \mathrm{Ct}(\mathrm{AG})=26.45-19.91=6.54$

$\Delta \Delta \mathrm{Ct}=\Delta \mathrm{Ct}(\mathrm{AG})-\Delta \mathrm{Ct}$ (vehicle)

$\Delta \Delta \mathrm{Ct}=1.26$

$X=2^{-\Delta \Delta C}$

$X=0.41$ (Down regulated)

As down regulated $(\mathrm{x}<0.5)$; Fold change $=\frac{1}{X}$

Fold change $=-2.39$

\section{Round 2}

$\Delta \mathrm{Ct}($ Vehicle $)=$ Average LXR Ct- Average Act Ct $\quad \Delta \mathrm{Ct}(\mathrm{AG})=$ Average LXR Ct- AverageActCt

$\Delta \mathrm{Ct}($ Vehicle $)=26.79-21.16=5.63 \quad \Delta \mathrm{Ct}(\mathrm{AG})=29.43-22.03=7.40$

$\Delta \Delta \mathrm{Ct}=\Delta \mathrm{Ct}(\mathrm{AG})-\Delta \mathrm{Ct}$ (vehicle)

$\Delta \Delta \mathrm{Ct}=1.78$

$X=2^{-\Delta \Delta C t}$

$X=0.29$ (Down regulated)

As down regulated $(\mathrm{x}<0.5) ;$ Fold change $=\frac{1}{X}$

Fold change $=-3.42$

\section{Average Fold change and variation}

Fold Change $=-2.39( \pm 0.7)$ 


\section{Hyperglycaemic environment at 34 hours}

\section{Vehicle v Acyl-ghrelin (AG)}

\section{Round 1}

$\Delta \mathrm{Ct}($ Vehicle $)=$ Average LXR Ct- Average Act Ct $\quad \Delta \mathrm{Ct}(\mathrm{AG})=$ Average LXR Ct- AverageActCt

$\Delta \mathrm{Ct}($ Vehicle $)=25.49-19.72=5.76 \quad \Delta \mathrm{Ct}(\mathrm{AG})=27.39-17.90=9.49$

$\Delta \Delta \mathrm{Ct}=\Delta \mathrm{Ct}(\mathrm{AG})-\Delta \mathrm{Ct}$ (vehicle)

$\Delta \Delta \mathrm{Ct}=3.72$

$$
\begin{aligned}
& X=2^{-\Delta \Delta C t} \\
& X=0.08 \text { (Down regulated) }
\end{aligned}
$$

As down regulated $(\mathrm{x}<0.5) ;$ Fold change $=\frac{1}{X}$

Fold change $=-13.2$

\section{Round 2}

$\Delta \mathrm{Ct}$ (Vehicle) $=$ Average LXR Ct- Average Act Ct $\quad \Delta \mathrm{Ct}(\mathrm{AG})=$ Average LXR Ct- AverageActCt

$\Delta \mathrm{Ct}($ Vehicle $)=25.26-17.64=7.61 \quad \Delta \mathrm{Ct}(\mathrm{AG})=29.31-18.61=10.70$

$$
\begin{aligned}
& \Delta \Delta \mathrm{Ct}=\Delta \mathrm{Ct}(\mathrm{AG})-\Delta \mathrm{Ct} \text { (vehicle) } \\
& \Delta \Delta \mathrm{Ct}=3.09 \\
& \mathrm{X}=2^{-\Delta \Delta \mathrm{Ct}} \\
& \mathrm{X}=0.29 \text { (Down regulated) }
\end{aligned}
$$

As down regulated $(\mathrm{x}<0.5)$; Fold change $=\frac{1}{X}$

Fold change $=-8.5$

\section{Average Fold change and variation}

Fold Change $=-10.84( \pm 3.4)$ 


\section{Normoglycaemic environment at 17 hours}

\section{Vehicle v Acyl-ghrelin (AG)}

\section{Round 1}

$\Delta \mathrm{Ct}($ Vehicle $)=$ Average LXR Ct- Average Act Ct $\quad \Delta \mathrm{Ct}(\mathrm{AG})=$ Average LXR Ct- AverageActCt

$\Delta \mathrm{Ct}($ Vehicle $)=25.70-24.50=1.20$

$\Delta \mathrm{Ct}(\mathrm{AG})=22.41-21.08=1.33$

$$
\begin{aligned}
& \Delta \Delta \mathrm{Ct}=\Delta \mathrm{Ct}(\mathrm{AG})-\Delta \mathrm{Ct} \text { (vehicle) } \\
& \Delta \Delta \mathrm{Ct}=-0.13 \\
& \mathrm{X}=2^{-\Delta \Delta \mathrm{Ct}} \\
& \mathrm{X}=1.10 \text { (No change) }
\end{aligned}
$$

\section{Round 2}

$\Delta \mathrm{Ct}($ Vehicle $)=$ Average LXR Ct- Average Act Ct $\quad \Delta \mathrm{Ct}(\mathrm{AG})=$ Average LXR Ct- AverageActCt

$\Delta \mathrm{Ct}($ Vehicle $)=22.82-20.86=1.96$

$\Delta \mathrm{Ct}(\mathrm{AG})=23.93-22.52=1.41$

$$
\begin{aligned}
& \Delta \Delta \mathrm{Ct}=\Delta \mathrm{Ct}(\text { acylG) }-\Delta \mathrm{Ct} \text { (vehicle) } \\
& \Delta \Delta \mathrm{Ct}=0.55 \\
& \mathrm{X}=2^{-\Delta \Delta \mathrm{Ct}} \\
& \mathrm{X}=0.68 \text { (No change) }
\end{aligned}
$$

\section{Average Fold change and variation}

Fold Change $=0.89( \pm 0.29)$ 


\section{Normoglycaemic environment at 34 hours}

\section{Vehicle v Acyl-ghrelin (AG)}

\section{Round 1}

$\Delta \mathrm{Ct}($ Vehicle $)=$ Average LXR Ct - Average Act Ct $\quad \Delta \mathrm{Ct}(\mathrm{AG})=$ Average LXR Ct- AverageActCt

$\Delta \mathrm{Ct}($ Vehicle $)=24.76-21.32=3.43$

$\Delta \mathrm{Ct}(\mathrm{AG})=25.60-24.07=1.53$

$$
\begin{aligned}
& \Delta \Delta \mathrm{Ct}=\Delta \mathrm{Ct}(\text { acylG })-\Delta \mathrm{Ct} \text { (vehicle) } \\
& \Delta \Delta \mathrm{Ct}=-1.90 \\
& X=2^{-\Delta \Delta \mathrm{Ct}} \\
& X=3.74 \text { (Up regulation) }
\end{aligned}
$$

\section{Round 2}

$\Delta \mathrm{Ct}$ (Vehicle)= Average LXR Ct- Average Act Ct $\quad \Delta \mathrm{Ct}(\mathrm{AG})=$ Average LXR Ct- AverageActCt

$\Delta \mathrm{Ct}($ Vehicle $)=25.14-21.32=3.82$

$\Delta \mathrm{Ct}(\mathrm{AG})=27.04-25.29=1.75$

$$
\begin{aligned}
& \Delta \Delta \mathrm{Ct}=\Delta \mathrm{Ct}(\mathrm{AG})-\Delta \mathrm{Ct} \text { (vehicle) } \\
& \Delta \Delta \mathrm{Ct}=-1.75 \\
& \mathrm{X}=2^{-\Delta \Delta \mathrm{Ct}} \\
& \mathrm{X}=3.36 \text { (Up regulation) }
\end{aligned}
$$

\section{Average Fold change and variation}

Fold Change $=+3.55( \pm 0.27)$ 


\section{$\Delta \triangle C t$ Values for ABCG1}

\section{Hyperglycaemic environment at 17 hours}

\section{Vehicle v Acyl-ghrelin (AG)}

\section{Round 1}

$\Delta \mathrm{Ct}$ (Vehicle) $=$ Average $\mathrm{ABC} \mathrm{Ct}-$ Average Act $\mathrm{Ct} \quad \Delta \mathrm{Ct}(\mathrm{AG})=$ Average $\mathrm{ABC} \mathrm{Ct}-$ AverageActCt

$\Delta \mathrm{Ct}$ (Vehicle $)=27.63-20.39=7.24$

$\Delta \mathrm{Ct}(\mathrm{AG})=28.23-19.91=8.32$

$$
\begin{aligned}
& \Delta \Delta \mathrm{Ct}=\Delta \mathrm{Ct}(\mathrm{AG})-\Delta \mathrm{Ct} \text { (vehicle) } \\
& \Delta \Delta \mathrm{Ct}=1.09 \\
& \mathrm{X}=2^{-\Delta \Delta \mathrm{Ct}} \\
& \mathrm{X}=0.47 \text { (Down regulated) }
\end{aligned}
$$

As down regulated $(\mathrm{x}<0.5)$; Fold change $=\frac{1}{X}$

Fold change $=-2.12$

\section{Round 2}

$\Delta \mathrm{Ct}($ Vehicle $)=$ Average $\mathrm{ABC} \mathrm{Ct}-$ Average Act $\mathrm{Ct} \quad \Delta \mathrm{Ct}(\mathrm{AG})=$ Average $\mathrm{ABC} \mathrm{Ct}-$ AverageActCt $\Delta \mathrm{Ct}($ Vehicle $)=28.73-21.16=7.56 \quad \Delta \mathrm{Ct}(\mathrm{AG})=31.06-22.03=9.04$

$$
\begin{aligned}
& \Delta \Delta \mathrm{Ct}=\Delta \mathrm{Ct}(\text { acylG) }-\Delta \mathrm{Ct} \text { (vehicle) } \\
& \Delta \Delta \mathrm{Ct}=1.48 \\
& \mathrm{X}=2^{-\Delta \Delta \mathrm{Ct}} \\
& \mathrm{X}=0.36 \text { (Down regulated) }
\end{aligned}
$$

As down regulated $(\mathrm{x}<0.5)$; Fold change $=\frac{1}{X}$

Fold change $=-2.78$

\section{Average Fold change and variation}

Fold Change $=-2.45( \pm 0.5)$ 


\section{Hyperglycaemic environment at 34 hours}

\section{Vehicle v Acyl-ghrelin (AG)}

\section{Round 1}

$\Delta \mathrm{Ct}$ (Vehicle) $=$ Average $\mathrm{ABC} \mathrm{Ct}-$ Average Act $\mathrm{Ct} \quad \Delta \mathrm{Ct}(\mathrm{AG})=$ Average $\mathrm{ABC} \mathrm{Ct}-$ AverageActCt

$\Delta \mathrm{Ct}($ Vehicle $)=28.84-19.72=9.12$

$\Delta \mathrm{Ct}(\mathrm{AG})=29.98-17.90=12.08$

$\Delta \Delta \mathrm{Ct}=\Delta \mathrm{Ct}(\mathrm{AG})-\Delta \mathrm{Ct}$ (vehicle)

$\Delta \Delta \mathrm{Ct}=2.96$

$$
\begin{aligned}
& X=2^{-\Delta \Delta C t} \\
& X=0.13 \text { (Down regulated) }
\end{aligned}
$$

As down regulated $(\mathrm{x}<0.5) ;$ Fold change $=\frac{1}{X}$

Fold change $=-7.80$

\section{Round 2}

$\Delta \mathrm{Ct}($ Vehicle $)=$ Average $\mathrm{ABC} \mathrm{Ct}-$ Average Act $\mathrm{Ct} \quad \Delta \mathrm{Ct}(\mathrm{AG})=$ Average $\mathrm{ABC} \mathrm{Ct}-$ AverageActCt

$\Delta \mathrm{Ct}($ Vehicle $)=25.80-17.64=8.16 \quad \Delta \mathrm{Ct}(\mathrm{AG})=28.98-18.61=10.37$

$\Delta \Delta \mathrm{Ct}=\Delta \mathrm{Ct}(\mathrm{AG})-\Delta \mathrm{Ct}$ (vehicle)

$\Delta \Delta \mathrm{Ct}=2.21$

$X=2^{-\Delta \Delta c t}$

$X=0.22$ (Down regulated)

As down regulated $(\mathrm{x}<0.5)$; Fold change $=\frac{1}{X}$

Fold change $=-4.64$

\section{Average Fold change and variation}

Fold Change $=-6.22( \pm 2.2)$ 


\section{Normoglycaemic environment at 17 hours}

\section{Vehicle v Acyl-ghrelin (AG)}

\section{Round 1}

$\Delta \mathrm{Ct}($ Vehicle $)=$ Average $\mathrm{ABC} \mathrm{Ct}-$ Average Act Ct $\Delta \mathrm{Ct}(\mathrm{AG})=$ Average $\mathrm{ABC} \mathrm{Ct}-$ Average ActCt

$\Delta \mathrm{Ct}($ Vehicle $)=27.73-24.50=3.23 \quad \Delta \mathrm{Ct}(\mathrm{AG})=27.58-21.08=6.50$

$\Delta \Delta \mathrm{Ct}=\Delta \mathrm{Ct}(\mathrm{AG})-\Delta \mathrm{Ct}$ (vehicle)

$\Delta \Delta \mathrm{Ct}=3.27$

$X=2^{-\Delta \Delta C t}$

$X=0.10$ (Down regulated)

As down regulated $(\mathrm{x}<0.5) ;$ Fold change $=\frac{1}{X}$

Fold change $=-9.64$

\section{Round 2}

$\Delta \mathrm{Ct}($ Vehicle $)=$ Average $\mathrm{ABC} \mathrm{Ct}-$ Average Act Ct $\Delta \mathrm{Ct}(\mathrm{AG})=$ Average $\mathrm{ABC} \mathrm{Ct}-$ Average ActCt $\Delta \mathrm{Ct}($ Vehicle $)=24.82-20.86=3.96 \quad \Delta \mathrm{Ct}(\mathrm{AG})=29.20-22.52=6.68$

$\Delta \Delta \mathrm{Ct}=\Delta \mathrm{Ct}(\mathrm{AG})-\Delta \mathrm{Ct}$ (vehicle)

$\Delta \Delta \mathrm{Ct}=2.72$

$X=2^{-\Delta \Delta C t}$

$X=0.15$ (Down regulated)

As down regulated $(\mathrm{x}<0.5)$; Fold change $=\frac{1}{X}$

Fold change $=-6.60$

\section{Average Fold change and variation}

Fold Change $=-8.12( \pm 2.1)$ 


\section{Normoglycaemic environment at 34 hours}

\section{Vehicle v Acyl-ghrelin (AG)}

\section{Round 1}

$\Delta \mathrm{Ct}$ (Vehicle) $=$ Average $\mathrm{ABC} \mathrm{Ct}-$ Average Act $\mathrm{Ct} \quad \Delta \mathrm{Ct}(\mathrm{AG})=$ Average $\mathrm{ABC} \mathrm{Ct}-$ Average ActCt

$\Delta \mathrm{Ct}($ Vehicle $)=27.31-21.32=5.99$ $\Delta \mathrm{Ct}(\mathrm{AG})=29.49-24.07=5.42$

$$
\begin{aligned}
& \Delta \Delta \mathrm{Ct}=\Delta \mathrm{Ct}(\mathrm{AG})-\Delta \mathrm{Ct} \text { (vehicle) } \\
& \Delta \Delta \mathrm{Ct}=0.57 \\
& \mathrm{X}=2^{-\Delta \Delta \mathrm{Ct}} \\
& \mathrm{X}=0.67 \text { (No change) }
\end{aligned}
$$

\section{Round 2}

$\Delta \mathrm{Ct}($ Vehicle $)=$ Average $\mathrm{ABC} \mathrm{Ct}-$ Average Act $\mathrm{Ct} \quad \Delta \mathrm{Ct}(\mathrm{AG})=$ Average $\mathrm{ABC} \mathrm{Ct}-$ Average ActCt

$\Delta \mathrm{Ct}($ Vehicle $)=27.62-21.32=6.30$ $\Delta \mathrm{Ct}(\mathrm{AG})=31.33-25.29=6.04$

$$
\begin{aligned}
& \Delta \Delta \mathrm{Ct}=\Delta \mathrm{Ct}(\mathrm{AG})-\Delta \mathrm{Ct} \text { (vehicle) } \\
& \Delta \Delta \mathrm{Ct}=-0.3 \\
& \mathrm{X}=2^{-\Delta \Delta \mathrm{Ct}} \\
& \mathrm{X}=1.20 \text { (No change) }
\end{aligned}
$$

\section{Average Fold change and variation}

Fold Change $=0.94( \pm 0.4)$ 


\section{$\Delta \Delta$ Ct Values for SREBF1}

\section{Hyperglycaemic environment at 17 hours}

\section{Vehicle v Acyl-ghrelin (AG)}

\section{Round 1}

$\Delta \mathrm{Ct}($ Vehicle $)=$ Average SRB Ct- Average Act Ct $\quad \Delta \mathrm{Ct}(\mathrm{AG})=$ Average SRB Ct- Average ActCt

$\Delta \mathrm{Ct}($ Vehicle $)=35.12-20.39=14.72 \quad \Delta \mathrm{Ct}(\mathrm{AG})=34.21-19.91=14.30$

$$
\begin{aligned}
& \Delta \Delta \mathrm{Ct}=\Delta \mathrm{Ct}(\mathrm{AG})-\Delta \mathrm{Ct} \text { (vehicle) } \\
& \Delta \Delta \mathrm{Ct}=-0.42 \\
& \mathrm{X}=2^{-\Delta \Delta \mathrm{Ct}} \\
& \mathrm{X}=1.34 \text { (No change) }
\end{aligned}
$$

\section{Round 2}

$\Delta \mathrm{Ct}$ (Vehicle) $=$ Average SRB Ct- Average Act Ct $\quad \Delta \mathrm{Ct}(\mathrm{AG})=$ Average SRB Ct- Average ActCt $\Delta \mathrm{Ct}($ Vehicle $)=35.09-21.16=13.92 \quad \Delta \mathrm{Ct}(\mathrm{AG})=35.35-22.03=13.33$

$$
\begin{aligned}
& \Delta \Delta \mathrm{Ct}=\Delta \mathrm{Ct}(\mathrm{AG})-\Delta \mathrm{Ct} \text { (vehicle) } \\
& \Delta \Delta \mathrm{Ct}=-0.59 \\
& \mathrm{X}=2^{-\Delta \Delta \mathrm{Ct}} \\
& \mathrm{X}=1.50 \text { (No change) }
\end{aligned}
$$

\section{Average Fold change and variation}

Fold Change $=1.42( \pm 0.1)$ 


\section{Hyperglycaemic environment at 34 hours}

\section{Vehicle v Acyl-ghrelin (AG)}

\section{Round 1}

$\Delta \mathrm{Ct}$ (Vehicle) $=$ Average SRB Ct- Average Act Ct $\quad \Delta \mathrm{Ct}(\mathrm{AG})=$ Average SRB Ct- Average ActCt

$\Delta \mathrm{Ct}($ Vehicle $)=33.93-19.72=14.20$ $\Delta \mathrm{Ct}(\mathrm{AG})=32.47-17.90=14.57$

$$
\begin{aligned}
& \Delta \Delta \mathrm{Ct}=\Delta \mathrm{Ct}(\mathrm{AG})-\Delta \mathrm{Ct} \text { (vehicle) } \\
& \Delta \Delta \mathrm{Ct}=0.37 \\
& \mathrm{X}=2^{-\Delta \Delta \mathrm{Ct}} \\
& \mathrm{X}=0.77 \text { (No change) }
\end{aligned}
$$

\section{Round 2}

$\Delta \mathrm{Ct}($ Vehicle $)=$ Average SRB Ct- Average Act Ct $\quad \Delta \mathrm{Ct}(\mathrm{AG})=$ Average SRB Ct- Average ActCt $\Delta \mathrm{Ct}($ Vehicle $)=32.38-17.64=14.74 \quad \Delta \mathrm{Ct}(\mathrm{AG})=33.62-18.61=15.01$

$$
\begin{aligned}
& \Delta \Delta \mathrm{Ct}=\Delta \mathrm{Ct}(\mathrm{AG})-\Delta \mathrm{Ct} \text { (vehicle) } \\
& \Delta \Delta \mathrm{Ct}=-0.27 \\
& \mathrm{X}=2^{-\Delta \Delta \mathrm{Ct}} \\
& \mathrm{X}=1.20 \text { (No change) }
\end{aligned}
$$

\section{Average Fold change and variation}

Fold Change $=1.0( \pm 0.3)$ 


\section{Normoglycaemic environment at 17 hours}

\section{Vehicle v Acyl-ghrelin (AG)}

\section{Round 1}

$\Delta \mathrm{Ct}$ (Vehicle) $=$ Average SRB Ct- Average Act Ct $\quad \Delta \mathrm{Ct}(\mathrm{AG})=$ Average SRB Ct- Average ActCt

$\Delta \mathrm{Ct}($ Vehicle $)=34.99-24.50=10.49 \quad \Delta \mathrm{Ct}(\mathrm{AG})=32.51-21.08=11.44$

$\Delta \Delta \mathrm{Ct}=\Delta \mathrm{Ct}(\mathrm{AG})-\Delta \mathrm{Ct}$ (vehicle)

$$
\begin{aligned}
& \Delta \Delta \mathrm{Ct}=-0.95 \\
& \mathrm{X}=2^{-\Delta \Delta \mathrm{Ct}} \\
& \mathrm{X}=1.93 \text { (Up regulated) }
\end{aligned}
$$

\section{Round 2}

$\Delta \mathrm{Ct}($ Vehicle $)=$ Average SRB Ct- Average Act Ct $\quad \Delta \mathrm{Ct}(\mathrm{AG})=$ Average SRB Ct- Average ActCt

$\Delta \mathrm{Ct}($ Vehicle $)=31.55-20.86=10.69 \quad \Delta \mathrm{Ct}(\mathrm{AG})=33.98-22.52=11.46$

$$
\begin{aligned}
& \Delta \Delta \mathrm{Ct}=\Delta \mathrm{Ct}(\mathrm{AG})-\Delta \mathrm{Ct} \text { (vehicle) } \\
& \Delta \Delta \mathrm{Ct}=-0.77 \\
& \mathrm{X}=2^{-\Delta \Delta \mathrm{Ct}} \\
& \mathrm{X}=1.71 \text { (Up regulated) }
\end{aligned}
$$

\section{Average Fold change and variation}

Fold Change $=+1.82( \pm 0.2)$ 


\section{Normoglycaemic environment at 34 hours}

\section{Vehicle v Acyl-ghrelin (AG)}

\section{Round 1}

$\Delta \mathrm{Ct}$ (Vehicle) $=$ Average SRB Ct- Average Act Ct $\quad \Delta \mathrm{Ct}(\mathrm{AG})=$ Average SRB Ct- Average ActCt

$\Delta \mathrm{Ct}($ Vehicle $)=33.68-21.32=12.35$

$\Delta \mathrm{Ct}(\mathrm{AG})=32.51-24.07=11.44$

$$
\begin{aligned}
& \Delta \Delta \mathrm{Ct}=\Delta \mathrm{Ct}(\mathrm{AG})-\Delta \mathrm{Ct} \text { (vehicle) } \\
& \Delta \Delta \mathrm{Ct}=-2.22 \\
& \mathrm{X}=2^{-\Delta \Delta \mathrm{Ct}} \\
& \mathrm{X}=4.65 \text { (Up regulated) }
\end{aligned}
$$

\section{Round 2}

$\Delta \mathrm{Ct}($ Vehicle $)=$ Average SRB Ct- Average Act Ct $\quad \Delta \mathrm{Ct}(\mathrm{AG})=$ Average SRB Ct- Average ActCt

$\Delta \mathrm{Ct}($ Vehicle $)=33.78-21.32=12.46$

$\Delta \mathrm{Ct}(\mathrm{AG})=34.91-25.29=9.62$

$$
\begin{aligned}
& \Delta \Delta \mathrm{Ct}=\Delta \mathrm{Ct}(\mathrm{AG})-\Delta \mathrm{Ct} \text { (vehicle) } \\
& \Delta \Delta \mathrm{Ct}=-2.84 \\
& \mathrm{X}=2^{-\Delta \Delta \mathrm{Ct}} \\
& \mathrm{X}=7.14 \text { (Up regulated) }
\end{aligned}
$$

\section{Average Fold change and variation}

Fold Change $=+5.90( \pm 1.8)$ 
Appendix 6

Raw $\Delta \Delta C T$ data for hVAT

Data used in Chapter 4 


\section{B-actin referred to as Act throughout}

\section{$\underline{A B C G 1}$}

\section{Non-obese v Obese}

$\Delta \mathrm{Ct}(\mathrm{NO})=$ AverageABCG1Ct- AverageActCt $\Delta \mathrm{Ct}(\mathrm{O})=$ AverageABCG1Ct - AverageActCt

$\Delta \mathrm{Ct}(\mathrm{NO})=26.49-18.79=7.70 \quad \Delta \mathrm{Ct}(\mathrm{O})=26.70-19.04=7.95$

$$
\begin{aligned}
& \Delta \Delta \mathrm{Ct}=\Delta \mathrm{Ct}(\mathrm{O})-\Delta \mathrm{Ct}(\mathrm{NO}) \\
& \Delta \Delta \mathrm{Ct}=0.25 \\
& \mathrm{X}=2^{-\Delta \Delta \mathrm{Ct}} \\
& \mathrm{X}=0.84 \text { (no change) }
\end{aligned}
$$

\section{Non-obese v OT2D}

$\Delta \mathrm{Ct}(\mathrm{NO})=$ AverageABCG1Ct-AverageActCt $\Delta \mathrm{Ct}(\mathrm{OT} 2 \mathrm{D})=$ AverageABCG1Ct-AverageActCt

$\Delta \mathrm{Ct}(\mathrm{NO})=26.49-18.79=7.70$

$\Delta \mathrm{Ct}(\mathrm{OT} 2 \mathrm{D})=27.80-18.83=8.97$

$\Delta \Delta \mathrm{Ct}=\Delta \mathrm{Ct}(\mathrm{OT} 2 \mathrm{DM})-\Delta \mathrm{Ct}(\mathrm{NO})$

$\Delta \Delta \mathrm{Ct}=1.27$

$$
\begin{aligned}
& X=2^{-\Delta \Delta C t} \\
& X=0.41 \text { (down regulated) }
\end{aligned}
$$

As down regulated $(\mathrm{x}<0.5)$; Fold change $=\frac{1}{X}$

Fold change $=-2.44$

\section{Obese (0) v OT2DM}

$\Delta \mathrm{Ct}(\mathrm{O})=$ AverageABCG1Ct-AverageActCt $\quad \Delta \mathrm{Ct}(\mathrm{OT} 2 \mathrm{D})=$ AverageABCG1Ct- AverageActCt

$\Delta \mathrm{Ct}(\mathrm{O})=26.70-19.04=7.95 \quad \Delta \mathrm{Ct}(\mathrm{OT} 2 \mathrm{D})=27.80-18.83=8.97$

$$
\begin{aligned}
& \Delta \Delta \mathrm{Ct}=\Delta \mathrm{Ct}(\mathrm{OT} 2 \mathrm{D})-\Delta \mathrm{Ct}(\mathrm{O}) \\
& \Delta \Delta \mathrm{Ct}=1.02 \\
& \mathrm{X}=2^{-\Delta \Delta \mathrm{Ct}} \\
& \mathrm{X}=0.49 \text { (down regulated) }
\end{aligned}
$$

As down regulated $(\mathrm{x}<0.5)$; Fold change $=\frac{1}{X}$

Fold change $=-2.04$ 


\section{Obesity effect}

\section{NO v Obese (O+OT2D)}

$\Delta \mathrm{Ct}(\mathrm{NO})=$ AverageABCG1Ct- AverageActCt $\quad \Delta \mathrm{Ct}(\mathrm{O}+\mathrm{OT} 2 \mathrm{D})=$ AverageABCG1Ct-AverageActCt

$\Delta \mathrm{Ct}(\mathrm{NO})=26.49-18.79=7.70$

$\Delta \mathrm{Ct}=8.36$

$$
\begin{aligned}
& \Delta \Delta \mathrm{Ct}=\Delta \mathrm{Ct}(\mathrm{O}+\mathrm{OT} 2 \mathrm{D})-\Delta \mathrm{Ct}(\mathrm{NO}) \\
& \Delta \Delta \mathrm{Ct}=0.66 \\
& \mathrm{X}=2^{-\Delta \Delta C \mathrm{t}} \\
& \mathrm{X}=0.63 \text { (no change) }
\end{aligned}
$$

\section{Diabetes effect}

\section{No Diabetes (NO+O) v OT2D}

$\Delta \mathrm{Ct}(\mathrm{NO}=\mathrm{O})=$ AverageABCG1Ct- AverageActCt $\quad \Delta \mathrm{Ct}(\mathrm{OT} 2 \mathrm{D})=$ AverageABCG1Ct-AverageActCt

$\mathrm{Ct}=7.82$ $\Delta \mathrm{Ct}=8.96$

$\Delta \Delta \mathrm{Ct}=\Delta \mathrm{Ct}(\mathrm{OT} 2 \mathrm{D})-\Delta \mathrm{Ct}(\mathrm{NO}+\mathrm{O})$

$\Delta \Delta \mathrm{Ct}=1.14$

$$
\begin{aligned}
& X=2^{-\Delta \Delta C t} \\
& X=0.45 \text { (down regulated) }
\end{aligned}
$$

As down regulated $(x<0.5)$; Fold change $=\frac{1}{X}$

Fold change $=-2.22$ 


\section{$\underline{\operatorname{LXR} \beta}$}

\section{Non-obese v Obese}

$\Delta \mathrm{Ct}(\mathrm{NO})=$ Average LXRßCt- AverageActCt

$\Delta \mathrm{Ct}(\mathrm{O})=$ AverageLXRßCt- AverageActCt

$\Delta \mathrm{Ct}(\mathrm{NO})=25.89-18.79=7.10$

$\Delta \mathrm{Ct}(\mathrm{O})=26.59-19.04=7.55$

$\Delta \Delta \mathrm{Ct}=\Delta \mathrm{Ct}(\mathrm{O})-\Delta \mathrm{Ct}(\mathrm{NO})$

$\Delta \Delta \mathrm{Ct}=0.45$

$X=2^{-\Delta \Delta C t}$

$X=0.73$ (no change)

\section{Non-obese v OT2D}

$\Delta \mathrm{Ct}(\mathrm{NO})=$ Average LXR $\beta C \mathrm{Ct}-$ AverageActCt $\quad \Delta \mathrm{Ct}(\mathrm{OT} 2 \mathrm{D})=$ AverageLXRBCt- AverageActCt

$\Delta \mathrm{Ct}(\mathrm{NO})=25.89-18.79=7.10 \quad \Delta \mathrm{Ct}(\mathrm{OT} 2 \mathrm{D})=27.72-19.15=8.57$

$\Delta \Delta \mathrm{Ct}=\Delta \mathrm{Ct}(\mathrm{OT} 2 \mathrm{D})-\Delta \mathrm{Ct}(\mathrm{NO})$

$\Delta \Delta \mathrm{Ct}=1.47$

$X=2^{-\Delta \Delta c t}$

$X=0.36$ (down regulated)

As down regulated $(\mathrm{x}<0.5) ;$ Fold change $=\frac{1}{X}$

Fold change $=-2.78$

\section{Obese v OT2D}

$\Delta \mathrm{Ct}(\mathrm{O})=$ Average LXR $\beta \mathrm{Ct}-$ AverageActCt $\quad \Delta \mathrm{Ct}(\mathrm{OT} 2 \mathrm{D})=$ Average LXR $\beta \mathrm{Ct}-$ AverageActCt

$\Delta \mathrm{Ct}(\mathrm{O})=26.59-19.04=7.55 \quad \Delta \mathrm{Ct}(\mathrm{OT} 2 \mathrm{D})=27.72-19.15=8.57$

$\Delta \Delta \mathrm{Ct}=\Delta \mathrm{Ct}(\mathrm{OT} 2 \mathrm{D})-\Delta \mathrm{Ct}(\mathrm{O})$

$\Delta \Delta \mathrm{Ct}=1.02$

$X=2^{-\Delta \Delta C t}$

$X=0.49$ (down regulated)

As down regulated $(x<0.5)$; Fold change $=\frac{1}{X}$

Fold change $=-2.04$ 


\section{Obesity effect}

\section{Non-obese v Obese (O+OT2D)}

$\Delta \mathrm{Ct}(\mathrm{NO})=$ AverageLXRBCt- AverageActCt $\quad \Delta \mathrm{Ct}(\mathrm{O}+\mathrm{OT} 2 \mathrm{D})=$ AverageLXRßCt- AverageActCt

$\Delta \mathrm{Ct}$ (leans) $=7.14$

$\Delta \mathrm{Ct}(\mathrm{O}+\mathrm{OT} 2 \mathrm{D})=26.59-19.04=8.13$

$\Delta \Delta \mathrm{Ct}=\Delta \mathrm{Ct}(\mathrm{O}+\mathrm{OT} 2 \mathrm{D})-\Delta \mathrm{Ct}(\mathrm{NO})$

$\Delta \Delta \mathrm{Ct}=0.99$

$X=2^{-\Delta \Delta C t}$

$X=0.50$ (no change)

\section{Diabetes effect}

(NO+O) v OT2D

$\Delta \mathrm{Ct}(\mathrm{NO}+\mathrm{O})=$ AverageLXRBCt- AverageActCt

$\Delta \mathrm{Ct}(\mathrm{OT} 2 \mathrm{D})=$ AverageLXRBCt- AverageActCt

$\Delta \mathrm{Ct}(\mathrm{NO}+\mathrm{O})=7.42$

$\Delta \mathrm{Ct}(\mathrm{OT} 2 \mathrm{D})=27.72-19.15=8.57$

$\Delta \Delta \mathrm{Ct}=\Delta \mathrm{Ct}(\mathrm{OT} 2 \mathrm{D})-\Delta \mathrm{Ct}(\mathrm{NO}+\mathrm{O})$

$\Delta \Delta \mathrm{Ct}=1.15$

$X=2^{-\Delta \Delta C t}$

$X=0.45$ (down regulated)

As down regulated $(\mathrm{x}<0.5) ;$ Fold change $=\frac{1}{X}$

Fold change $=-2.22$ 


\section{PPARY}

\section{Non-obese v Obese}

$\Delta \mathrm{Ct}(\mathrm{NO})=$ AveragePPAR $\gamma \mathrm{Ct}-$ AverageActCt $\quad \Delta \mathrm{Ct}(\mathrm{O})=$ AveragePPAR $\gamma \mathrm{Ct}-$ AverageActCt

$\Delta \mathrm{Ct}(\mathrm{NO})=2.84$

$$
\Delta \mathrm{Ct}(\mathrm{O})=3.30
$$

$$
\begin{aligned}
& \Delta \Delta \mathrm{Ct} \Delta \mathrm{Ct}(\mathrm{O})-\Delta \mathrm{Ct}(\mathrm{NO}) \\
& \Delta \Delta \mathrm{Ct}=0.46 \\
& \mathrm{X}=2^{-\Delta \Delta \mathrm{Ct}} \\
& \mathrm{X}=0.73 \text { (no change) }
\end{aligned}
$$

\section{Non-obese v OT2DM}

$\Delta \mathrm{Ct}(\mathrm{NO})=$ AveragePPAR $\gamma \mathrm{Ct}-$ AverageActCt $\quad \Delta \mathrm{Ct}(\mathrm{OT} 2 \mathrm{D})=$ AveragePPAR $\gamma \mathrm{Ct}-\mathrm{AverageActCt}$

$\Delta \mathrm{Ct}(\mathrm{NO})=2.84$ $\Delta \mathrm{Ct}(\mathrm{OT} 2 \mathrm{D})=3.44$

$$
\begin{aligned}
& \Delta \Delta \mathrm{Ct}=\Delta \mathrm{Ct}(\mathrm{OT} 2 \mathrm{D})-\Delta \mathrm{Ct}(\mathrm{NO}) \\
& \Delta \Delta \mathrm{Ct}=0.6 \\
& \mathrm{X}=2^{-\Delta \Delta \mathrm{Ct}} \\
& \mathrm{X}=0.66 \text { (no change) }
\end{aligned}
$$

\section{Obese v OT2D}

$\Delta \mathrm{Ct}(\mathrm{O})=$ AveragePPAR $\gamma \mathrm{Ct}-\mathrm{AverageActCt} \quad \Delta \mathrm{Ct}(\mathrm{OT} 2 \mathrm{D})=$ AveragePPAR $\gamma \mathrm{Ct}-\mathrm{AverageActCt}$

$\Delta \mathrm{Ct}(\mathrm{O})=3.30$ $\Delta \mathrm{Ct}(\mathrm{OT} 2 \mathrm{D})=3.44$

$\Delta \Delta \mathrm{Ct}=\Delta \mathrm{Ct}(\mathrm{OT} 2 \mathrm{DM})-\Delta \mathrm{Ct}$ (obese)

$\Delta \Delta \mathrm{Ct}=0.14$

$X=2^{-\Delta \Delta C t}$

$X=0.91$ (no change) 


\section{Obesity effect}

\section{Non-obese v Obese (O+OT2D)}

$\Delta \mathrm{Ct}(\mathrm{NO})=$ AveragePPAR $\gamma \mathrm{Ct}-$ AverageActCt $\quad \Delta \mathrm{Ct}(\mathrm{O}+\mathrm{OT} 2 \mathrm{D})=$ AveragePPAR $\gamma \mathrm{Ct}-$ AverageActCt

$\Delta \mathrm{Ct}(\mathrm{NO})=2.84$ $\Delta \mathrm{Ct}(\mathrm{O}+\mathrm{OT} 2 \mathrm{D})=3.37$

$\Delta \Delta \mathrm{Ct} \Delta \mathrm{Ct}(\mathrm{O}+\mathrm{OT} 2 \mathrm{D})-\Delta \mathrm{Ct}(\mathrm{NO})$

$\Delta \Delta \mathrm{Ct}=0.53$

$X=2^{-\Delta \Delta C t}$

$X=0.69$ (no change)

\section{Diabetes effect}

\section{(NO+O) v OT2DM}

$\Delta \mathrm{Ct}(\mathrm{NO}+\mathrm{O})=$ AveragePPAR $\gamma \mathrm{Ct}-$ AverageActCt $\quad \Delta \mathrm{Ct}(\mathrm{OT} 2 \mathrm{D})=$ AveragePPAR $\gamma \mathrm{Ct}-$ AverageActCt

$\Delta \mathrm{Ct}(\mathrm{NO}+\mathrm{O})=3.07$

$\Delta \mathrm{Ct}(\mathrm{OT} 2 \mathrm{D})=3.44$

$\Delta \Delta \mathrm{Ct}=\Delta \mathrm{Ct}(\mathrm{OT} 2 \mathrm{D})-\Delta \mathrm{Ct}(\mathrm{NO}+\mathrm{O})$

$\Delta \Delta \mathrm{Ct}=0.37$

$X=2^{-\Delta \Delta C t}$

$X=0.77$ (no change) 


\section{SREBF1}

\section{Non-obese v Obese}

$\Delta \mathrm{Ct}(\mathrm{NO})=$ AverageSREBF1Ct - AverageActCt $\quad \Delta \mathrm{Ct}(\mathrm{O})=$ AverageSREBF1Ct - AerageActCt

$\Delta \mathrm{Ct}(\mathrm{NO})=27.61-18.79=8.82$

$\Delta \mathrm{Ct}(\mathrm{O})=27.70-19.04=8.66$

$\Delta \Delta \mathrm{Ct}=\Delta \mathrm{Ct}(\mathrm{O})-\Delta \mathrm{Ct}(\mathrm{NO})$

$\Delta \Delta \mathrm{Ct}=-0.16$

$X=2^{-\Delta \Delta C t}$

$X=1.12$ (no change)

\section{Non-obese v OT2D}

$\Delta \mathrm{Ct}(\mathrm{NO})=$ AverageSREBF1Ct-AverageActCt $\Delta \mathrm{Ct}(\mathrm{OT} 2 \mathrm{D})=$ AverageSREBF1Ct-averageActCt

$\Delta \mathrm{Ct}(\mathrm{INO})=27.61-18.79=8.82 \quad \Delta \mathrm{Ct}(\mathrm{OT} 2 \mathrm{D})=29.40-19.15=10.25$

$\Delta \Delta \mathrm{Ct}=\Delta \mathrm{Ct}(\mathrm{OT} 2 \mathrm{D})-\Delta \mathrm{Ct}(\mathrm{NO})$

$\Delta \Delta \mathrm{Ct}=1.43$

$X=2^{-\Delta \Delta C t}$

$X=0.37$ (down regulated)

As down regulated $(\mathrm{x}<0.5) ;$ Fold change $=\frac{1}{X}$

Fold change $=-2.70$

\section{Obese v OT2DM}

$\Delta \mathrm{Ct}(\mathrm{O})=$ AverageSREBF1Ct-AverageActCt $\quad \Delta \mathrm{Ct}(\mathrm{OT} 2 \mathrm{D})=$ AverageSREBF1Ct - AverageActCt

$\Delta \mathrm{Ct}(\mathrm{O})=27.70-19.04=8.66 \quad \Delta \mathrm{Ct}(\mathrm{OT} 2 \mathrm{D})=29.40-19.15=10.25$

$\Delta \Delta \mathrm{Ct}=\Delta \mathrm{Ct}(\mathrm{OT} 2 \mathrm{D})-\Delta \mathrm{Ct}(\mathrm{O})$

$\Delta \Delta \mathrm{Ct}=1.59$

$X=2^{-\Delta \Delta c t}$

$X=0.33$ (down regulated)

As down regulated $(\mathrm{x}<0.5)$; Fold change $=\frac{1}{X}$

Fold change $=-3.03$ 


\section{Obesity effect}

\section{Non-obese v Obese (O+OT2DM)}

$\Delta \mathrm{Ct}($ leans) $=$ AverageSREBF1Ct-AverageActCt $\Delta \mathrm{Ct}$ (Obese) $=$ AverageSREBF1Ct-AverageActCt

$\Delta \mathrm{Ct}$ (leans) $=27.61-18.79=8.82$ $\Delta \mathrm{Ct}($ Obese $)=27.70-19.04=9.46$

$\Delta \Delta \mathrm{Ct}=\Delta \mathrm{Ct}$ (Obese) $-\Delta \mathrm{Ct}$ (lean)

$\Delta \Delta \mathrm{Ct}=0.64$

$X=2^{-\Delta \Delta C t}$

$X=0.64$ (no change)

\section{(NO+O) v OT2DM}

$\Delta \mathrm{Ct}(\mathrm{NO}+\mathrm{O})=$ AverageSREBF1Ct-AverageActCt $\Delta \mathrm{Ct}(\mathrm{OT} 2 \mathrm{D})=$ AverageSREBF1Ct-AverageActCt $\Delta \mathrm{Ct}(\mathrm{NO}+\mathrm{O})=8.74$ $\Delta \mathrm{Ct}(\mathrm{OT} 2 \mathrm{D})=29.40-19.15=10.26$

$\Delta \Delta \mathrm{Ct}=\Delta \mathrm{Ct}(\mathrm{OT} 2 \mathrm{D})-\Delta \mathrm{Ct}(\mathrm{NO}+\mathrm{O})$

$\Delta \Delta \mathrm{Ct}=1.52$

$X=2^{-\Delta \Delta C t}$

$X=0.35$ (down regulated)

As down regulated $(\mathrm{x}<0.5)$; Fold change $=\frac{1}{X}$

Fold change $=-2.86$ 


\section{Ghrelin}

\section{Non-obese v Obese}

$\Delta \mathrm{Ct}(\mathrm{NO})=$ Average Ghrel Ct- Average Act $\mathrm{Ct}$

$\Delta \mathrm{Ct}(\mathrm{NO})=30.29-18.50=11.38$

$\Delta \Delta \mathrm{Ct}=\Delta \mathrm{Ct}(\mathrm{O})-\Delta \mathrm{Ct}(\mathrm{NO})$

$\Delta \Delta \mathrm{Ct}=-0.66$

$X=2^{-\Delta \Delta c t}$

$X=1.58$ (up regulation)

Fold change $=1.58$

\section{Non-obese v OT2D}

$\Delta \mathrm{Ct}(\mathrm{NO})=$ AverageGhrelCt-AverageActCt

$\Delta \mathrm{Ct}(\mathrm{NO})=30.29-18.50=11.38$

$\Delta \Delta \mathrm{Ct}=\Delta \mathrm{Ct}(\mathrm{OT} 2 \mathrm{D})-\Delta \mathrm{Ct}(\mathrm{NO})$

$\Delta \Delta \mathrm{Ct}=0.78$

$X=2^{-\Delta \Delta C t}$

$X=0.58$ (no change)
$\Delta \mathrm{Ct}(\mathrm{OT} 2 \mathrm{D})=$ AverageGhrelCt-AverageActCt

$\Delta \mathrm{Ct}(\mathrm{OT} 2 \mathrm{D})=31.14-19.10=12.13$

\section{Obese v OT2D}

$\Delta \mathrm{Ct}(\mathrm{O})=$ AverageGhrelCt-AverageActCt

$\Delta \mathrm{Ct}(\mathrm{OT} 2 \mathrm{D})=$ AverageGhrelCt-AverageActCt

$\Delta \mathrm{Ct}(\mathrm{O})=29.77-19.04=10.72$

$\Delta \mathrm{Ct}(\mathrm{OT} 2 \mathrm{D})=31.14-19.10=12.13$

$\Delta \Delta \mathrm{Ct}=\Delta \mathrm{Ct}(\mathrm{OT} 2 \mathrm{D})-\Delta \mathrm{Ct}(\mathrm{O})$

$\Delta \Delta \mathrm{Ct}=1.41$

$$
\begin{aligned}
& X=2^{-\Delta \Delta C t} \\
& X=0.38 \text { (down regulated) }
\end{aligned}
$$

As down regulated $(\mathrm{x}<0.5)$; Fold change $=\frac{1}{X}$

Fold change $=-2.6$ 


\section{Obesity effect}

\section{Non-obese v Obese (O+OT2D)}

$\Delta \mathrm{Ct}(\mathrm{NO})=$ AverageGhrelCt- AverageActCt

$\Delta \mathrm{Ct}(\mathrm{NO})=30.29-18.50=11.38$

$\Delta \Delta \mathrm{Ct}=\Delta \mathrm{Ct}(\mathrm{O})-\Delta \mathrm{Ct}$ (lean)

$\Delta \Delta \mathrm{Ct}=0.05$

$X=2^{-\Delta \Delta c t}$

$X=0.97$ (no change)
$\Delta \mathrm{Ct}(\mathrm{O}+\mathrm{OT} 2 \mathrm{D})=$ AverageGhrelCt-AverageActCt

$\Delta \mathrm{Ct}(\mathrm{O}+\mathrm{OT} 2 \mathrm{D})=11.43$

\section{Diabetes effect}

(NO+O) v OT2D

$\Delta \mathrm{Ct}(\mathrm{NO}+\mathrm{O})=$ AverageGhrelCt-AverageActCt $\quad \Delta \mathrm{Ct}(\mathrm{OT} 2 \mathrm{D})=$ AverageGhrelCt-AverageActCt

$\Delta \mathrm{Ct}(\mathrm{NO}+\mathrm{O})=11.05$

$\Delta \mathrm{Ct}(\mathrm{OT} 2 \mathrm{D})=31.14-19.10=12.13$

$\Delta \Delta \mathrm{Ct}=\Delta \mathrm{Ct}(\mathrm{OT} 2 \mathrm{D})-\Delta \mathrm{Ct}(\mathrm{NO}+\mathrm{O})$

$\Delta \Delta \mathrm{Ct}=1.08$

$X=2^{-\Delta \Delta C t}$

$X=0.47$ (down regulated)

As down regulated $(\mathrm{x}<0.5) ;$ Fold change $=\frac{1}{X}$

Fold change $=-2.1$ 
mBOAT4 (GOAT)

\section{Non-obese v Obese}

$\Delta \mathrm{Ct}(\mathrm{NO})=$ AverageGOATCt-AverageActCt

$\Delta \mathrm{Ct}(\mathrm{NO})=10.05$

$\Delta \Delta \mathrm{Ct}=\Delta \mathrm{Ct}(\mathrm{O})-\Delta \mathrm{Ct}(\mathrm{NO})$

$\Delta \Delta \mathrm{Ct}=0.18$

$X=2^{-\Delta \Delta c t}$

$X=0.88$ (no change)
$\Delta \mathrm{Ct}(\mathrm{O})=$ AverageGOATCt-AverageActCt

$\Delta \mathrm{Ct}(\mathrm{O})=10.23$

\section{Non-obese v OT2D}

$\Delta \mathrm{Ct}(\mathrm{NO})=$ AverageGOATCt-AverageActCt

$\Delta \mathrm{Ct}(\mathrm{NO})=10.05$

$\Delta \mathrm{Ct}(\mathrm{OT} 2 \mathrm{D})=$ AverageGOATCt- Average $\mathrm{ActCt}$

$\Delta \mathrm{Ct}(\mathrm{OT} 2 \mathrm{D})=10.64$

$\Delta \Delta \mathrm{Ct}=\Delta \mathrm{Ct}(\mathrm{OT} 2 \mathrm{D})-\Delta \mathrm{Ct}(\mathrm{NO})$

$\Delta \Delta \mathrm{Ct}=0.59$

$X=2^{-\Delta \Delta C t}$

$X=0.66$ (no change)

\section{Obese v OT2D}

$\Delta \mathrm{Ct}(\mathrm{O})=$ AverageGOATCt-AverageActCt $\quad \Delta \mathrm{Ct}(\mathrm{OT} 2 \mathrm{D})=$ AverageGOATCt-AverageActCt

$\Delta \mathrm{Ct}(\mathrm{O})=10.23$

$\Delta \mathrm{Ct}(\mathrm{OT} 2 \mathrm{D})=10.64$

$\Delta \Delta \mathrm{Ct}=\Delta \mathrm{Ct}(\mathrm{OT} 2 \mathrm{D})-\Delta \mathrm{Ct}(\mathrm{O})$

$\Delta \Delta \mathrm{Ct}=0.41$

$X=2^{-\Delta \Delta C t}$

$X=0.75$ (no change) 


\section{Obesity effect}

\section{Non-obese v Obese (O+OT2D)}

$\Delta \mathrm{Ct}(\mathrm{NO})=$ AverageGOATCt-AverageActCt $\quad \Delta \mathrm{Ct}(\mathrm{O}+\mathrm{OT} 2 \mathrm{D})=$ AverageGOATCt-AverageActCt

$\Delta \mathrm{Ct}(\mathrm{NO})=10.05$ $\Delta \mathrm{Ct}(\mathrm{O}+\mathrm{OT} 2 \mathrm{D})=10.44$

$\Delta \Delta \mathrm{Ct}=\Delta \mathrm{Ct}(\mathrm{O}+\mathrm{OT} 2 \mathrm{D})-\Delta \mathrm{Ct}(\mathrm{NO})$

$\Delta \Delta \mathrm{Ct}=0.39$

$X=2^{-\Delta \Delta C t}$

$X=0.76$ (no change)

\section{Diabetes effect}

(NO+O) v OT2D

$\Delta \mathrm{Ct}(\mathrm{NO}+\mathrm{O})=$ AverageGOATCt-AverageActCt

$\Delta \mathrm{Ct}(\mathrm{OT} 2 \mathrm{D})=$ AverageGOATCt$-\mathrm{AverageActCt}$

$\Delta \mathrm{Ct}(\mathrm{NO}+\mathrm{O})=10.15$

$\Delta \mathrm{Ct}(\mathrm{OT} 2 \mathrm{D})=10.64$

$\Delta \Delta \mathrm{Ct}=\Delta \mathrm{Ct}(\mathrm{OT} 2 \mathrm{D})-\Delta \mathrm{Ct}(\mathrm{NO}+\mathrm{O})$

$\Delta \Delta \mathrm{Ct}=0.49$

$X=2^{-\Delta \Delta C t}$

$X=0.71$ (no change) 
Lypla

\section{Non-obese v Obese}

$\Delta \mathrm{Ct}(\mathrm{NO})=$ AverageLyplaCt - AverageActCt $\Delta \mathrm{Ct}(\mathrm{O})=$ Average LyplaCt- AverageActCt

$\Delta \mathrm{Ct}(\mathrm{NO})=24.40-18.79=5.61$ $\Delta \mathrm{Ct}(\mathrm{O})=18.79-19.04=5.07$

$\Delta \Delta \mathrm{Ct}=\Delta \mathrm{Ct}(\mathrm{O})-\Delta \mathrm{Ct}(\mathrm{NO})$

$\Delta \Delta \mathrm{Ct}=-0.54$

$X=2^{-\Delta \Delta c t}$

$X=1.45$ (no change)

\section{Non-obese v OT2D}

$\Delta \mathrm{Ct}(\mathrm{NO})=$ AverageLyplaCt - AverageActCt

$\Delta \mathrm{Ct}(\mathrm{OT} 2 \mathrm{D})=$ AverageLyplaCt-AverageActCt

$\Delta \mathrm{Ct}(\mathrm{NO})=24.40-18.79=5.61$

$\Delta \mathrm{Ct}(\mathrm{OT} 2 \mathrm{D})=24.58-19.15=5.43$

$\Delta \Delta \mathrm{Ct}=\Delta \mathrm{Ct}(\mathrm{OT} 2 \mathrm{D})-\Delta \mathrm{Ct}(\mathrm{NO})$

$\Delta \Delta \mathrm{Ct}=-0.18$

$X=2^{-\Delta \Delta C t}$

$X=1.13$ (no change)

\section{Obese v OT2D}

$\Delta \mathrm{Ct}(\mathrm{O})=$ AverageLyplaCt-AverageActCt $\quad \Delta \mathrm{Ct}(\mathrm{OT} 2 \mathrm{D})=$ AverageLyplaCt-AverageActCt

$\Delta \mathrm{Ct}(\mathrm{O})=18.79-19.04=5.07 \quad \Delta \mathrm{Ct}(\mathrm{OT} 2 \mathrm{D})=24.58-19.15=5.43$

$\Delta \Delta \mathrm{Ct}=\Delta \mathrm{Ct}(\mathrm{OT} 2 \mathrm{D})-\Delta \mathrm{Ct}(\mathrm{O})$

$\Delta \Delta \mathrm{Ct}=0.36$

$X=2^{-\Delta \Delta C t}$

$X=0.78$ (no change) 


\section{Obesity effect}

\section{Non-obese v Obese (O+OT2D)}

$\Delta \mathrm{Ct}(\mathrm{NO})=$ AverageLyplaCt - AverageActCt $\Delta \mathrm{Ct}(\mathrm{O}+\mathrm{OT} 2 \mathrm{D})=$ Average LyplaCt-AverageActCt

$\Delta \mathrm{Ct}(\mathrm{NO})=24.40-18.79=5.61$ $\Delta \mathrm{Ct}(\mathrm{O}+\mathrm{OT} 2 \mathrm{D})=5.25$

$$
\begin{aligned}
& \Delta \Delta \mathrm{Ct}=\Delta \mathrm{Ct}(\mathrm{O}+\mathrm{OT} 2 \mathrm{D})-\Delta \mathrm{Ct}(\mathrm{NO}) \\
& \Delta \Delta \mathrm{Ct}=-0.36 \\
& \mathrm{X}=2^{-\Delta \Delta \mathrm{Ct}} \\
& \mathrm{X}=1.28 \text { (no change) }
\end{aligned}
$$

\section{Diabetes effect}

\section{$(\mathrm{NO}+\mathrm{O})$ v OT2D}

$\Delta \mathrm{Ct}(\mathrm{NO}+\mathrm{O})=$ AverageLyplaCt - AverageActCt $\Delta \mathrm{Ct}(\mathrm{OT} 2 \mathrm{D})=$ AverageLyplaCt - AverageActCt $\Delta \mathrm{Ct}(\mathrm{NO}+\mathrm{O})=5.34$ $\Delta \mathrm{Ct}(\mathrm{OT} 2 \mathrm{D})=24.58-19.15=5.43$

$$
\begin{aligned}
& \Delta \Delta \mathrm{Ct}=\Delta \mathrm{Ct}(\mathrm{OT} 2 \mathrm{D})-\Delta \mathrm{Ct}(\mathrm{NO}+\mathrm{O}) \\
& \Delta \Delta \mathrm{Ct}=0.09 \\
& \mathrm{X}=2^{-\Delta \Delta \mathrm{Ct}} \\
& \mathrm{X}=0.94 \text { (no change) }
\end{aligned}
$$


Appendix 7

Raw $\Delta \Delta C T$ data for SGBS cells with metformin treatment

Data used in Chapter 5 


\section{$\underline{\Delta C t \text { Values for Metformin }}$}

\section{Vehicle (V) V Metformin (Met) treatment}

\section{$\Delta \Delta C$ t Values for LXR $\beta$}

\section{Round 1}

$\Delta \mathrm{Ct}(\mathrm{V})=$ Average LXR Ct- Average Act Ct

$\Delta \mathrm{Ct}(\mathrm{V})=25.68-20.39=5.28$

$$
\begin{aligned}
& \Delta \Delta \mathrm{Ct}=\Delta \mathrm{Ct}(\text { Met })-\Delta \mathrm{Ct}(\mathrm{V}) \\
& \Delta \Delta \mathrm{Ct}=-1.76 \\
& \mathrm{X}=2^{-\Delta \Delta \mathrm{Ct}} \\
& \mathrm{X}=3.39 \text { (Up regulated) }
\end{aligned}
$$

\section{Round 2}

$\Delta \mathrm{Ct}(\mathrm{V})=$ Average LXR Ct- Average Act Ct

$\Delta \mathrm{Ct}(\mathrm{V})=26.79-21.16=5.63$

$$
\begin{aligned}
& \Delta \Delta \mathrm{Ct}=\Delta \mathrm{Ct}(\text { Met })-\Delta \mathrm{Ct}(\mathrm{V}) \\
& \Delta \Delta \mathrm{Ct}=-1.83 \\
& \mathrm{X}=2^{-\Delta \Delta \mathrm{Ct}} \\
& \mathrm{X}=3.56 \text { (Up regulated) }
\end{aligned}
$$

\section{Average Fold change and variation}

Fold Change $=3.47( \pm 0.1)$
$\Delta \mathrm{Ct}($ Met $)=$ Average LXR Ct- AverageActCt

$\Delta \mathrm{Ct}($ Met $)=26.11-22.59=3.52$

$\Delta \mathrm{Ct}(\mathrm{Met})=$ Average LXR Ct- AverageActCt

$\Delta \mathrm{Ct}($ Met $)=25.61-21.81=3.80$ 


\section{$\triangle \triangle C t$ Values for ABCG1}

\section{Round 1}

$\Delta \mathrm{Ct}(\mathrm{V})=$ Average $\mathrm{ABC} \mathrm{Ct}$ - Average Act $\mathrm{Ct}$

$\Delta \mathrm{Ct}($ Met $)=$ Average $\mathrm{ABC} \mathrm{Ct}-$ Average $\mathrm{ActCt}$

$\Delta \mathrm{Ct}(\mathrm{V})=27.63-20.39=7.24$

$\Delta \mathrm{Ct}($ Met $)=24.53-22.59=1.94$

$$
\begin{aligned}
& \Delta \Delta C \mathrm{Ct}=\Delta \mathrm{Ct}(\text { Met })-\Delta \mathrm{Ct}(\mathrm{V}) \\
& \Delta \Delta \mathrm{Ct}=-5.3 \\
& \mathrm{X}=2^{-\Delta \Delta \mathrm{Ct}} \\
& \mathrm{X}=39.40 \text { (Up regulated) }
\end{aligned}
$$

\section{Round 2}

$\Delta \mathrm{Ct}(\mathrm{V})=$ Average $\mathrm{ABC} \mathrm{Ct}-$ Average $\mathrm{Act} \mathrm{Ct}$

$\Delta \mathrm{Ct}(\mathrm{Met})=$ Average $\mathrm{ABC} \mathrm{Ct}-$ Average $\mathrm{ActCt}$

$\Delta \mathrm{Ct}(\mathrm{V})=28.73-21.16=7.57$

$\Delta \mathrm{Ct}($ Met $)=24.18-21.81=2.37$

$$
\begin{aligned}
& \Delta \Delta \mathrm{Ct}=\Delta \mathrm{Ct} \text { (Met) }-\Delta \mathrm{Ct}(\mathrm{V}) \\
& \Delta \Delta \mathrm{Ct}=-5.2 \\
& \mathrm{X}=2^{-\Delta \Delta \mathrm{Ct}} \\
& \mathrm{X}=36.76 \text { (Up regulated) }
\end{aligned}
$$

\section{Average Fold change and variation}

Fold Change $=38.08( \pm 1.9)$ 


\section{$\Delta \Delta C$ t Values for SREBF1}

\section{Round 1}

$\Delta \mathrm{Ct}(\mathrm{V})=$ Average SRB Ct- Average Act Ct

$\Delta \mathrm{Ct}($ Met $)=$ Average SRB Ct- AverageActCt

$\Delta \mathrm{Ct}(\mathrm{V})=35.21-20.39=14.82$

$\Delta \mathrm{Ct}($ Met $)=35.45-22.59=12.86$

$$
\begin{aligned}
& \Delta \Delta C \mathrm{t}=\Delta \mathrm{Ct}(\text { Met })-\Delta \mathrm{Ct}(\mathrm{V}) \\
& \Delta \Delta \mathrm{Ct}=-1.96 \\
& X=2^{-\Delta \Delta \mathrm{Ct}} \\
& X=3.89 \text { (Up regulated) }
\end{aligned}
$$

\section{Round 2}

$$
\begin{aligned}
& \Delta \mathrm{Ct}(\mathrm{V})=\text { Average SRB Ct- Average Act Ct } \\
& \Delta \mathrm{Ct}(\mathrm{V})=35.09-21.16=13.92 \\
& \Delta \Delta \mathrm{Ct}=\Delta \mathrm{Ct} \text { (Met) }-\Delta \mathrm{Ct}(\mathrm{V}) \\
& \Delta \Delta \mathrm{Ct}=-1.49 \\
& \mathrm{X}=2^{-\Delta \Delta \mathrm{Ct}} \\
& \mathrm{X}=2.81 \text { (Up regulated) }
\end{aligned}
$$

$\Delta \mathrm{Ct}(\mathrm{Met})=$ Average SRB Ct- AverageActCt $\Delta \mathrm{Ct}($ Met $)=34.24-21.82=12.43$

\section{Average Fold change and variation}

Fold Change $=3.35( \pm 0.8)$ 


\section{Metfromin (Met) V Met and AG treatment}

\section{$\underline{\Delta C \mathrm{Ct} \text { Values for LXR } \beta}$}

\section{Round 1}

$\Delta \mathrm{Ct}($ Met $)=$ Average LXR Ct- Average Act Ct $\quad \Delta \mathrm{Ct}($ Met $+\mathrm{AG})=$ Average LXR Ct- AverageActCt

$\Delta C t($ Met $)=26.11-22.59=3.52 \quad \Delta C t($ Met $+A G)=28.20-19.71=8.49$

$$
\begin{aligned}
& \Delta \Delta \mathrm{Ct}=\Delta \mathrm{Ct}(\text { Met }+\& \mathrm{AG})-\Delta \mathrm{Ct} \text { (Met) } \\
& \Delta \Delta \mathrm{Ct}=4.97 \\
& \mathrm{X}=2^{-\Delta \Delta \mathrm{Ct}} \\
& \mathrm{X}=0.03 \text { (Down regulated) }
\end{aligned}
$$

As down regulated $(\mathrm{x}<0.5)$; Fold change $=\frac{1}{X}$

Fold change $=-31.3$

\section{Round 2}

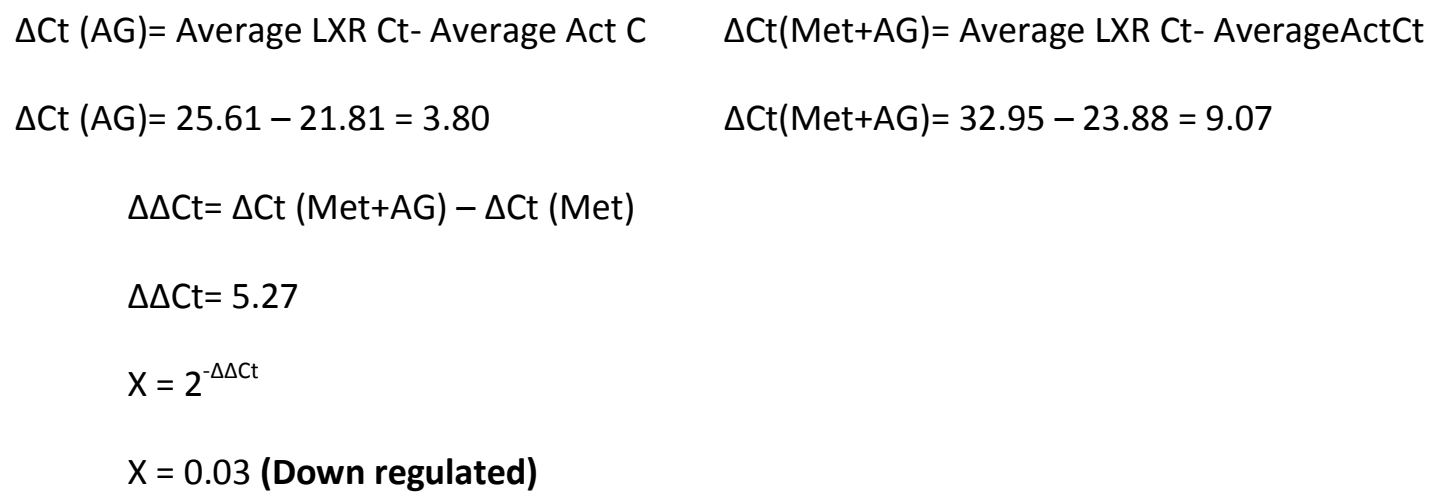

As down regulated $(\mathrm{x}<0.5) ;$ Fold change $=\frac{1}{X}$

Fold change $=-38.6$

\section{Average Fold change and variation}

Fold Change $=-34.2( \pm 4.0)$ 


\section{$\Delta \Delta C t$ Values for ABCG1}

\section{Round 1}

$\Delta \mathrm{Ct}(\mathrm{Met})=$ Average $\mathrm{ABC} \mathrm{Ct}-$ Average Act $\mathrm{Ct} \quad \Delta \mathrm{Ct}(\mathrm{Met}+\mathrm{AG})=$ Average $\mathrm{ABC} \mathrm{Ct}-$ AverageActCt

$\Delta \mathrm{Ct}($ Met $)=24.53-22.59=1.94 \quad \Delta \mathrm{Ct}($ Met $+\mathrm{AG})=26.82-19.71=7.11$

$\Delta \Delta \mathrm{Ct}=\Delta \mathrm{Ct}(\mathrm{Met}+\mathrm{AG})-\Delta \mathrm{Ct}(\mathrm{Met})$

$\Delta \Delta \mathrm{Ct}=5.17$

$X=2^{-\Delta \Delta C t}$

$X=0.03$ (Downregulated)

As down regulated $(\mathrm{x}<0.5) ;$ Fold change $=\frac{1}{X}$

Fold change $=-36.0$

\section{Round 2}

$\Delta \mathrm{Ct}(\mathrm{Met})=$ Average $\mathrm{ABC} \mathrm{Ct}-$ Average Act Ct $\quad \Delta \mathrm{Ct}($ Met+AG)= Average ABC Ct- AverageActCt

$\Delta C t($ Met $)=24.18-21.81=2.37 \quad \Delta C t($ Met $+A G)=31.36-23.88=7.48$

$\Delta \Delta \mathrm{Ct}=\Delta \mathrm{Ct}(\mathrm{Met}+\mathrm{AG})-\Delta \mathrm{Ct}(\mathrm{Met})$

$\Delta \Delta \mathrm{Ct}=5.11$

$X=2^{-\Delta \Delta C t}$

$X=0.03$ (Downregulated)

As down regulated $(x<0.5)$; Fold change $=\frac{1}{X}$

Fold change $=-34.5$

\section{Average Fold change and variation}

Fold Change $=-35.1( \pm 0.9)$ 


\section{$\Delta \Delta C$ t Values for SREBF1}

\section{Round 1}

$\Delta \mathrm{Ct}($ Met $)=$ Average SRB Ct- Average Act C $\quad \Delta \mathrm{Ct}($ Met $+\mathrm{AG})=$ Average SRB Ct- AverageActCt

$\Delta \mathrm{Ct}($ Met $)=35.45-22.59=12.86$

$\Delta \mathrm{Ct}($ Met $+\mathrm{AG})=32.10-19.71=12.39$

$$
\begin{aligned}
& \Delta \Delta C \mathrm{t}=\Delta \mathrm{Ct} \text { (Met+AG) }-\Delta \mathrm{Ct} \text { (Met) } \\
& \Delta \Delta \mathrm{Ct}=-0.47 \\
& \mathrm{X}=2^{-\Delta \Delta \mathrm{Ct}} \\
& \mathrm{X}=0.72 \text { (No change) }
\end{aligned}
$$

\section{Round 2}

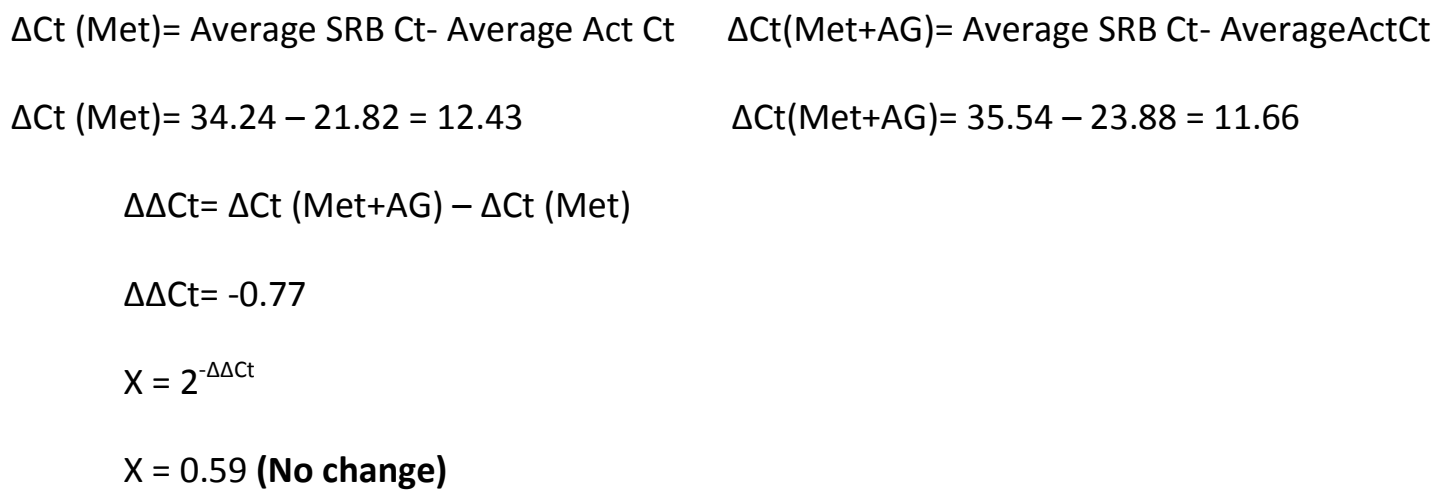

\section{Average Fold change and variation}

Fold Change $=0.65( \pm 0.2)$ 


\section{Acyl Ghrelin (AG) V Met and AG treatment}

\section{$\Delta \Delta C t$ Values for LXRß}

\section{Round 1}

$\Delta \mathrm{Ct}(\mathrm{AG})=$ Average LXR Ct- Average Act Ct $\quad \Delta \mathrm{Ct}($ Met+AG) $=$ Average LXR Ct- AverageActCt

$\Delta \mathrm{Ct}(\mathrm{AG})=26.45-19.91=6.54 \quad \Delta \mathrm{Ct}($ Met $+\mathrm{AG})=28.20-19.71=8.49$

$$
\begin{aligned}
& \Delta \Delta C t=\Delta C t(\text { Met }+\& A G)-\Delta C t(A G) \\
& \Delta \Delta C t=1.95 \\
& X=2^{-\Delta \Delta C t} \\
& X=0.26 \text { (Down regulated) }
\end{aligned}
$$

As down regulated $(\mathrm{x}<0.5) ;$ Fold change $=\frac{1}{X}$

Fold change $=-3.85$

\section{Round 2}

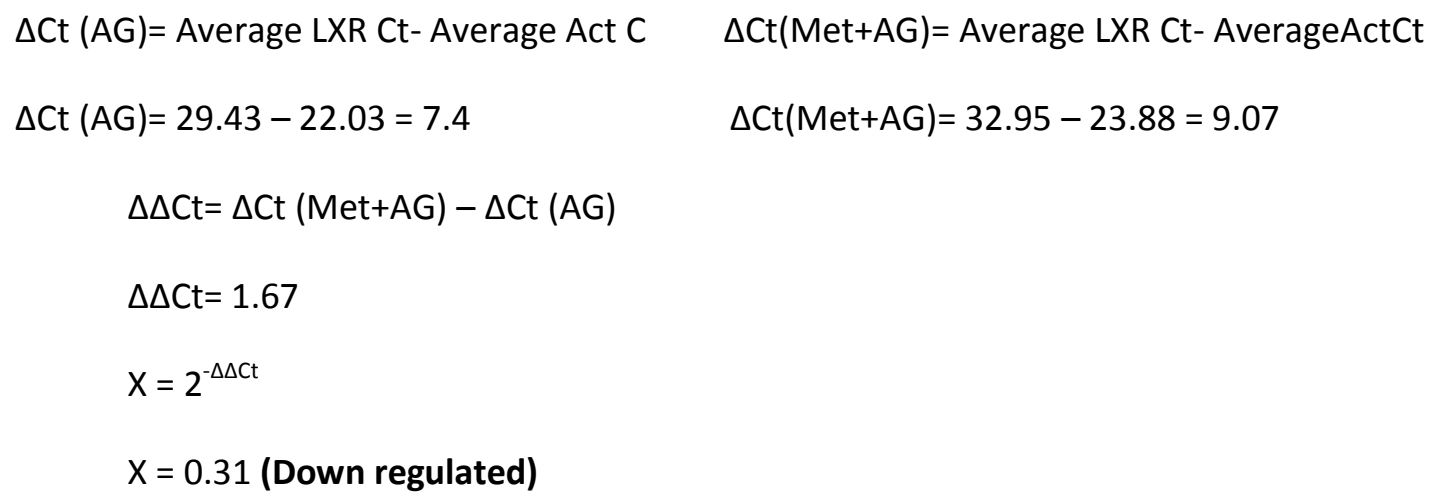

As down regulated $(\mathrm{x}<0.5) ;$ Fold change $=\frac{1}{X}$

Fold change $=-3.23$

\section{Average Fold change and variation}

Fold Change $=-3.54( \pm 0.4)$ 


\section{$\triangle \triangle C t$ Values for ABCG1}

\section{Round 1}

$\Delta \mathrm{Ct}(\mathrm{AG})=$ Average $\mathrm{ABC} \mathrm{Ct}-$ Average Act $\mathrm{Ct} \quad \Delta \mathrm{Ct}(\mathrm{Met}+\mathrm{AG})=$ Average ABC Ct- AverageActCt

$\Delta \mathrm{Ct}(\mathrm{AG})=28.24-19.91=8.33$ $\Delta C t($ Met $+A G)=26.82-19.71=7.11$

$$
\begin{aligned}
& \Delta \Delta \mathrm{Ct}=\Delta \mathrm{Ct}(\mathrm{Met}+\mathrm{AG})-\Delta \mathrm{Ct}(\mathrm{AG}) \\
& \Delta \Delta \mathrm{Ct}=-1.22 \\
& \mathrm{X}=2^{-\Delta \Delta \mathrm{Ct}} \\
& \mathrm{X}=2.33 \text { (Up regulated) }
\end{aligned}
$$

\section{Round 2}

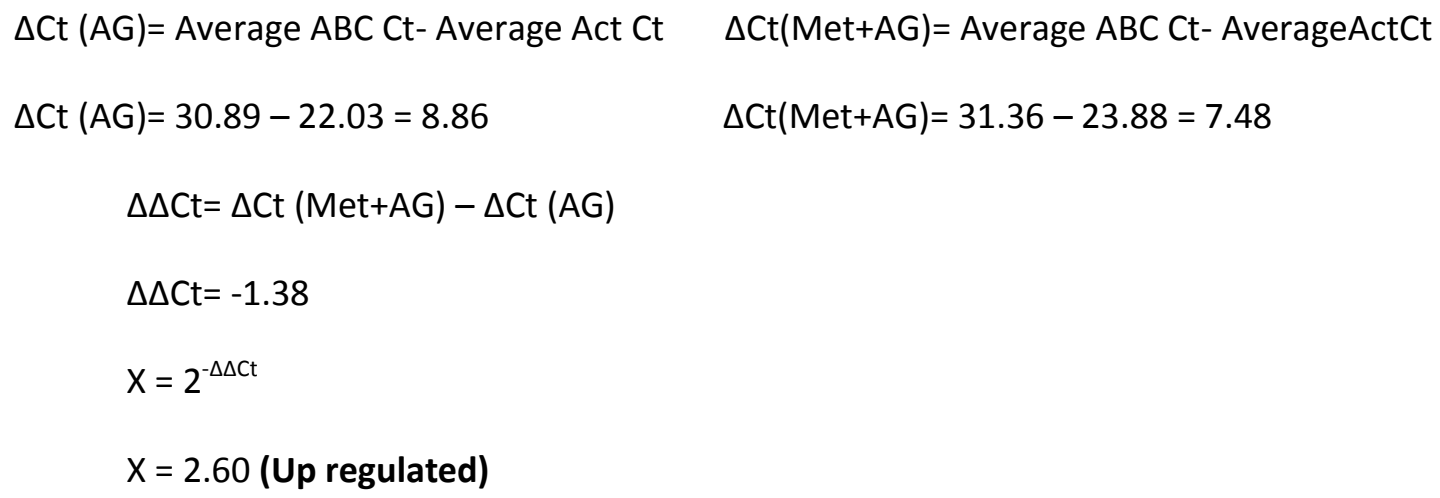

\section{Average Fold change and variation}

Fold Change $=2.47( \pm 0.2)$ 


\section{$\Delta \Delta C$ t Values for SREBF1}

\section{Round 1}

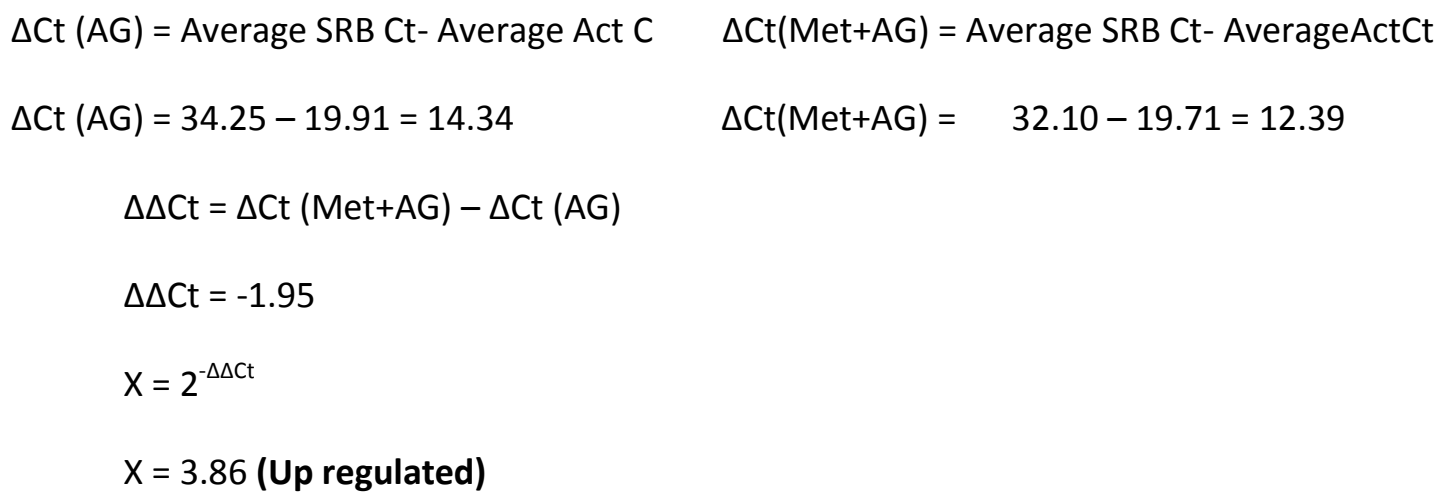

\section{Round 2}

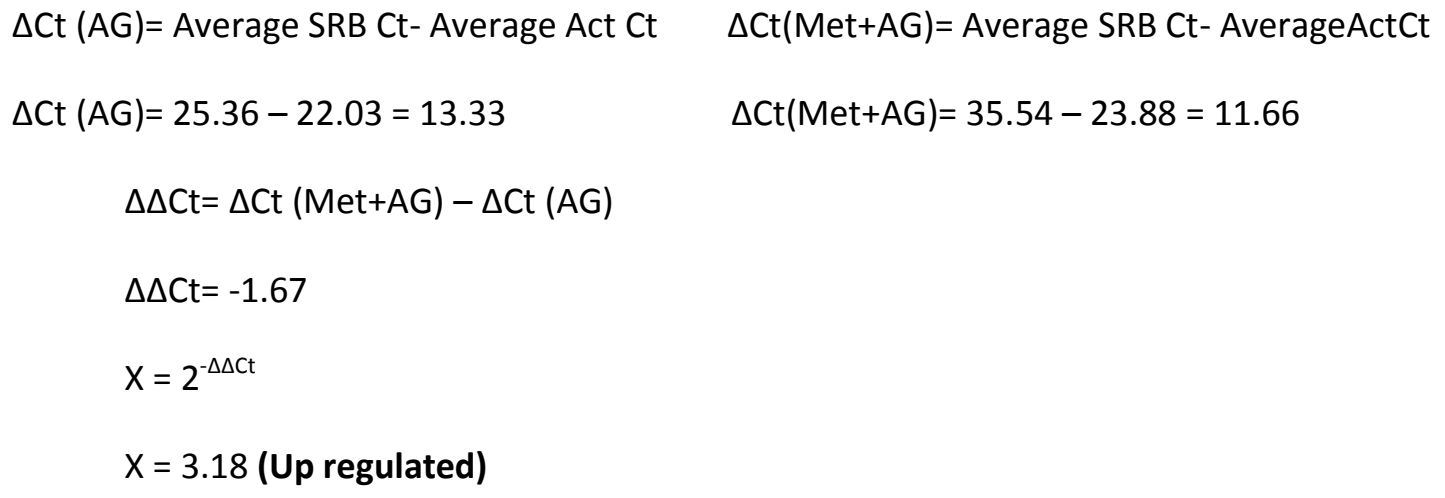

\section{Average Fold change and variation}

Fold Change $=3.52( \pm 0.5)$ 
Publication redacted due to copyright restrictions

\section{Appendix 8 \\ Publications}


Appendix 9

Conference lists 
British Obesity \& Metabolism Surgery Society January 2016- oral presentation

Churm R, Barry JD, Caplin S, Eyre N, Davies JS, Stephens JW \& Prior SL.

Cholesterol efflux in obesity related type 2 diabetes: Effects of glucose and ghrelin.

Diabetes UK March 2016- poster presentation

Churm R, Barry JD, Davies JS, Stephens JW \& Prior SL.

The effect of glucose on cholesterol efflux in obesity related Type 2 diabetes.

In Diabetic Medicine (Vol. 33, pp. 82-82)

WEDS May 2016- poster presentation

Churm R, Barry JD, Davies JS, Stephens JW \& Prior SL.

The effect of glucose on cholesterol efflux in obesity related Type 2 diabetes.

Diabetes UK March 2017- poster presentation

Churm R, Barry JD, Davies JS, Stephens JW \& Prior SL.

Effect of increased endogenous glucose levels within Type 2 diabetes on cellular lipid profiles

In Endocrine Abstracts (2017) 48 P2.

European Association of the Study of Diabetes (EASD) September 2017-poster presentation

Churm R, Caplin S, Barry J, Davies JS, Stephens JW, Prior SL

The role of acyl ghrelin in human visceral adipose tissue in relation to the LXR-ABC pathway and the metabolic state.

In DIABETOLOGIA (Vol. 60, pp. S270-S271). 
Bibliography 
AILHAUD, G. 1982. Adipose cell differentiation in culture. Molecular and cellular biochemistry, 49, 17-31.

AL-KHALILI, L., FORSGREN, M., KANNISTO, K., ZIERATH, J., LÖNNQVIST, F. \& KROOK, A. 2005. Enhanced insulin-stimulated glycogen synthesis in response to insulin, metformin or rosiglitazone is associated with increased mRNA expression of GLUT4 and peroxisomal proliferator activator receptor gamma co-activator 1. Diabetologia, 48, 1173-1179.

AMERICAN DIABETES ASSOCIATION 2016. 2. Classification and diagnosis of diabetes. Diabetes Care, 39, S13-S22.

AMERICAN DIABETES ASSOCIATION 2017. 7. Obesity management for the treatment of type 2 diabetes. Diabetes care, 40, S57-S63.

ANGELIDIS, G., VALOTASSIOU, V. \& GEORGOULIAS, P. 2010. Current and potential roles of ghrelin in clinical practice. Journal of endocrinological investigation, 33, 823-838.

ARONOFF, S. L., BERKOWITZ, K., SHREINER, B. \& WANT, L. 2004. Glucose metabolism and regulation: beyond insulin and glucagon. Diabetes Spectrum, 17, 183-190.

ASAKAWA, A., INUI, A., KAGA, T., KATSUURA, G., FUJIMIYA, M., FUJINO, M. \& KASUGA, M. 2003. Antagonism of ghrelin receptor reduces food intake and body weight gain in mice. Gut, 52, 947-952.

AU, C. C., DOCANTO, M. M., ZAHID, H., RAFFAELLI, F.-M., FERRERO, R. L., FURNESS, J. B. \& BROWN, K. A. 2017. Des-acyl ghrelin inhibits the capacity of macrophages to stimulate the expression of aromatase in breast adipose stromal cells. The Journal of steroid biochemistry and molecular biology, 170, 49-53. 
AYALA, A., MUÑOZ, M. F. \& ARGÜELLES, S. 2014. Lipid peroxidation: production, metabolism, and signaling mechanisms of malondialdehyde and 4-hydroxy-2nonenal. Oxidative medicine and cellular longevity, 2014.

AYDIN, S. 2015. A short history, principles, and types of ELISA, and our laboratory experience with peptide/protein analyses using ELISA. Peptides, 72, 4-15.

BAATAR, D., PATEL, K. \& TAUB, D. D. 2011. The effects of ghrelin on inflammation and the immune system. Molecular and cellular endocrinology, 340, 44-58.

BAI, J., YANG, F., DONG, L. \& ZHENG, Y. 2017. Ghrelin Protects Human Lens Epithelial Cells against Oxidative Stress-Induced Damage. Oxidative Medicine and Cellular Longevity, 2017.

BAILEY, C. J. 2017. Metformin: historical overview. Diabetologia, 60, 1566-1576.

BANKS, W. A., BURNEY, B. O. \& ROBINSON, S. M. 2008. Effects of triglycerides, obesity, and starvation on ghrelin transport across the blood-brain barrier. Peptides, 29, 20612065.

BANKS, W. A., TSCHÖP, M., ROBINSON, S. M. \& HEIMAN, M. L. 2002. Extent and direction of ghrelin transport across the blood-brain barrier is determined by its unique primary structure. Journal of Pharmacology and Experimental Therapeutics, 302, 822-827.

BARBATO, D. L., TATULLI, G., AQUILANO, K. \& CIRIOLO, M. 2013. FoxO1 controls lysosomal acid lipase in adipocytes: implication of lipophagy during nutrient restriction and metformin treatment. Cell death \& disease, 4, e861.

BASTARD, J.-P., MAACHI, M., LAGATHU, C., KIM, M. J., CARON, M., VIDAL, H., CAPEAU, J. \& FEVE, B. 2006. Recent advances in the relationship between obesity, inflammation, and insulin resistance. European cytokine network, 17, 4-12. 
BASTARD, J.-P., MAACHI, M., VAN NHIEU, J. T., JARDEL, C., BRUCKERT, E., GRIMALDI, A., ROBERT, J.-J., CAPEAU, J. \& HAINQUE, B. 2002. Adipose tissue IL-6 content correlates with resistance to insulin activation of glucose uptake both in vivo and in vitro. The Journal of Clinical Endocrinology \& Metabolism, 87, 2084-2089.

BAYS, H. E., GONZÁLEZ-CAMPOY, J. M., BRAY, G. A., KITABCHI, A. E., BERGMAN, D. A., SCHORR, A. B., RODBARD, H. W. \& HENRY, R. R. 2008. Pathogenic potential of adipose tissue and metabolic consequences of adipocyte hypertrophy and increased visceral adiposity. Expert review of cardiovascular therapy, 6, 343-368.

BEYNON, A. L., BROWN, M. R., WRIGHT, R., REES, M. I., SHELDON, I. M. \& DAVIES, J. S. 2013. Ghrelin inhibits LPS-induced release of IL-6 from mouse dopaminergic neurones. Journal of neuroinflammation, 10, 811.

BODEN, G. 1999. Free fatty acids, insulin resistance, and type 2 diabetes mellitus. Proceedings of the Association of American Physicians, 111, 241-248.

BORST, S. E. 2004. The role of TNF- $\alpha$ in insulin resistance. Endocrine, 23, 177-182.

BROWNER, W. S., NEWMAN, T. B. \& HULLEY, S. B. 2007. Estimating sample size and power: applications and examples. Designing clinical research, 3, 367.

CAI, H., CONG, W.-N., DAIMON, C. M., WANG, R., TSCHÖP, M. H., SÉVIGNY, J., MARTIN, B. \& MAUDSLEY, S. 2013. Altered lipid and salt taste responsivity in ghrelin and GOAT null mice. PLoS One, 8, e76553.

CALKIN, A. C. \& TONTONOZ, P. 2012. Transcriptional integration of metabolism by the nuclear sterol-activated receptors LXR and FXR. Nature reviews Molecular cell biology, 13, 213.

CAMERON, A. R., MORRISON, V., LEVIN, D., MOHAN, M., FORTEATH, C., BEALL, C., MCNEILlY, A. D., BALFOUR, D. J., SAVINKO, T. \& WONG, A. K. 2016. Anti- 
inflammatory effects of metformin irrespective of diabetes status. Circulation research, CIRCRESAHA. 116.308445.

CHABOT, F., CARON, A., LAPLANTE, M. \& ST-PIERRE, D. H. 2014. Interrelationships between ghrelin, insulin and glucose homeostasis: Physiological relevance. World journal of diabetes, 5, 328.

CHORNY, A., ANDERSON, P., GONZALEZ-REY, E. \& DELGADO, M. 2008. Ghrelin protects against experimental sepsis by inhibiting high-mobility group box 1 release and by killing bacteria. The Journal of Immunology, 180, 8369-8377.

CHURM, R., DAVIES, J., STEPHENS, J. \& PRIOR, S. 2017. Ghrelin function in human obesity and type 2 diabetes: a concise review. Obesity Reviews, 18, 140-148.

COOKE, A. A., CONNAUGHTON, R. M., LYONS, C. L., MCMORROW, A. M. \& ROCHE, H. M. 2016. Fatty acids and chronic low grade inflammation associated with obesity and the metabolic syndrome. European journal of pharmacology.

COOKE, M. S., EVANS, M. D., DIZDAROGLU, M. \& LUNEC, J. 2003. Oxidative DNA damage: mechanisms, mutation, and disease. The FASEB Journal, 17, 1195-1214.

COUSIN, B., CINTI, S., MORRONI, M., RAIMBAULT, S., RICQUIER, D., PENICAUD, L. \& CASTEILLA, L. 1992. Occurrence of brown adipocytes in rat white adipose tissue: molecular and morphological characterization. Journal of cell science, 103, 931-942.

CYPESS, A. M., LEHMAN, S., WILLIAMS, G., TAL, I., RODMAN, D., GOLDFINE, A. B., KUO, F. C., PALMER, E. L., TSENG, Y.-H. \& DORIA, A. 2009. Identification and importance of brown adipose tissue in adult humans. New England Journal of Medicine, 360, 15091517.

CYPESS, A. M., WeineR, L. S., ROBERTS-TOLER, C., ElíA, E. F., KESSLER, S. H., KAHN, P. A., ENGLiSh, J., CHATMAN, K., TRAUGER, S. A. \& DORIA, A. 2015. Activation of human 
brown adipose tissue by a $\beta 3$-adrenergic receptor agonist. Cell metabolism, 21, 3338.

DALEN, K. T., ULVEN, S. M., BAMBERG, K., GUSTAFSSON, J.-Å. \& NEBB, H. I. 2003. Expression of the insulin-responsive glucose transporter GLUT4 in adipocytes is dependent on liver X receptor $\alpha$. Journal of Biological Chemistry, 278, 48283-48291.

DARDZIŃSKA, J. A., MAŁGORZEWICZ, S., KASKA, Ł., PROCZKO, M., STEFANIAK, T., STANKIEWICZ, M. \& ŚLEDZIŃSKI, Z. 2014. Fasting and postprandial acyl and desacyl ghrelin levels in obese and non-obese subjects. Endokrynologia Polska, 65, 377-381.

DATE, Y., MURAKAMI, N., TOSHINAI, K., MATSUKURA, S., NIIJIMA, A., MATSUO, H., KANGAWA, K. \& NAKAZATO, M. 2002. The role of the gastric afferent vagal nerve in ghrelin-induced feeding and growth hormone secretion in rats. Gastroenterology, $123,1120-1128$.

DAVIES, J. S., KOTOKORPI, P., ECCLES, S. R., BARNES, S. K., TOKARCZUK, P. F., ALLEN, S. K., WHITWORTH, H. S., GUSCHINA, I. A., EVANS, B. A. \& MODE, A. 2009. Ghrelin induces abdominal obesity via GHS-R-dependent lipid retention. Molecular endocrinology, 23, 914-924.

DEBAUN, M. R., ESS, J. \& SAUNDERS, S. 2001. Simpson Golabi Behmel syndrome: progress toward understanding the molecular basis for overgrowth, malformation, and cancer predisposition. Molecular genetics and metabolism, 72, 279-286.

DELPARIGI, A., TSCHÖP, M., HEIMAN, M. L., SALBE, A. D., VOZAROVA, B., SELL, S. M., BUNT, J. C. \& TATARANNI, P. A. 2002. High circulating ghrelin: a potential cause for hyperphagia and obesity in Prader-Willi syndrome. The Journal of Clinical Endocrinology \& Metabolism, 87, 5461-5464. 
DEMERS, A., CARON, V., RODRIGUE-WAY, A., WAHLI, W., ONG, H. \& TREMBLAY, A. 2009. A concerted kinase interplay identifies PPARy as a molecular target of ghrelin signaling in macrophages.

DESJARDINS, P. \& CONKLIN, D. 2010. NanoDrop microvolume quantitation of nucleic acids. Journal of visualized experiments: JoVE.

DIECI, E., CASATI, L., PAGANI, F., CELOTTI, F. \& SIBILIA, V. 2014. Acylated and unacylated ghrelin protect MC3T3-E1 cells against tert-butyl hydroperoxide-induced oxidative injury: pharmacological characterization of ghrelin receptor and possible epigenetic involvement. Amino acids, 46, 1715-1725.

DIXIT, V. D., SCHAFFER, E. M., PYLE, R. S., COLLINS, G. D., SAKTHIVEL, S. K., PALANIAPPAN, R., LILLARD, J. W. \& TAUB, D. D. 2004. Ghrelin inhibits leptin-and activation-induced proinflammatory cytokine expression by human monocytes and T cells. The Journal of clinical investigation, 114, 57-66.

DUBOIS, S., HILL, D. E. \& BEATON, G. H. 1979. An examination of factors believed to be associated with infantile obesity. The American journal of clinical nutrition, 32, 19972004.

DUBOIS, S. G., HEILBRONN, L. K., SMITH, S. R., ALBU, J. B., KELLEY, D. E. \& RAVUSSIN, E. 2006. Decreased expression of adipogenic genes in obese subjects with type 2 diabetes. Obesity, 14, 1543-1552.

ESPOSITO, K., PONTILLO, A., GIUGLIANO, F., GIUGLIANO, G., MARFELLA, R., NICOLETTI, G. \& GIUGLIANO, D. 2003. Association of low interleukin-10 levels with the metabolic syndrome in obese women. The Journal of Clinical Endocrinology \& Metabolism, 88, 1055-1058. 
EVANS, J. L., GOLDFINE, I. D., MADDUX, B. A. \& GRODSKY, G. M. 2003. Are oxidative stressactivated signaling pathways mediators of insulin resistance and $\beta$-cell dysfunction? Diabetes, 52, 1-8.

EVANS, M., GRIFFITHS, H. \& LUNEC, J. 1997. Reactive oxygen species and their cytotoxic mechanisms. Advances in Molecular and Cell Biology, 20, 25-73.

FEDEle, D., COMI, G., COSCElli, C., CuCinotTA, D., FELdMAN, E. L., GHIRLANDA, G., GREENE, D. A., NEgRIN, P., SANTEUSANIO, F. \& COMMITTEE, I. D. N. 1997. A multicenter study on the prevalence of diabetic neuropathy in Italy. Diabetes Care, 20, 836-843.

FENKCl, V., FENKCI, S., YILMAZER, M. \& SERTESER, M. 2003. Decreased total antioxidant status and increased oxidative stress in women with polycystic ovary syndrome may contribute to the risk of cardiovascular disease. Fertility and sterility, 80, 123-127.

FERRE, P. \& FOUFELLE, F. 2007. SREBP-1c transcription factor and lipid homeostasis: clinical perspective. Hormone Research in Paediatrics, 68, 72-82.

FIELDING, C. J. \& FIELDING, P. E. 2001. Cellular cholesterol efflux. Biochimica et Biophysica Acta (BBA)-Molecular and Cell Biology of Lipids, 1533, 175-189.

FISCHER-POSOVSZKY, P., NEWELL, F. S., WABITSCH, M. \& TORNQVIST, H. E. 2008. Human SGBS cells-a unique tool for studies of human fat cell biology. Obesity facts, 1, 184189.

FOWLER, M. J. 2008. Microvascular and macrovascular complications of diabetes. Clinical diabetes, 26, 77-82.

FRITTITTA, L., YOUNGREN, J., SBRACCIA, P., D'ADAMO, M., BUONGIORNO, A., VIGNERI, R., GOLDFINE, I. \& TRISCHITTA, V. 1997. Increased adipose tissue PC-1 protein content, but not tumour necrosis factor-a gene expression, is associated with a reduction of 
both whole body insulin sensitivity and insulin receptor tyrosine-kinase activity. Diabetologia, 40, 282-289.

FUJITA, K., IWAMA, H., OURA, K., TADOKORO, T., HIROSE, K., WATANABE, M., SAKAMOTO, T., KATSURA, A., MIMURA, S. \& NOMURA, T. 2016. Metformin-suppressed differentiation of human visceral preadipocytes: Involvement of microRNAs. International journal of molecular medicine, 38, 1135-1140.

FUJITA, K., NISHIZAWA, H., FUNAHASHI, T., SHIMOMURA, I. \& SHIMABUKURO, M. 2006. Systemic oxidative stress is associated with visceral fat accumulation and the metabolic syndrome. Circulation Journal, 70, 1437-1442.

FURUKAWA, S., FUJITA, T., SHIMABUKURO, M., IWAKI, M., YAMADA, Y., NAKAJIMA, Y., NAKAYAMA, O., MAKISHIMA, M., MATSUDA, M. \& SHIMOMURA, I. 2017. Increased oxidative stress in obesity and its impact on metabolic syndrome. The Journal of clinical investigation, 114, 1752-1761.

GAGNON, J., SHEPPARD, E. \& ANINI, Y. 2013. Metformin directly inhibits ghrelin secretion through AMP-activated protein kinase in rat primary gastric cells. Diabetes, Obesity and Metabolism, 15, 276-279.

GARIN, M. C., BURNS, C. M., KAUL, S. \& CAPPOLA, A. R. 2013. The human experience with ghrelin administration. The Journal of Clinical Endocrinology \& Metabolism, 98, 18261837.

GHOSHAL, S., WITTA, J., ZHONG, J., DE VILLIERS, W. \& ECKHARDT, E. 2009. Chylomicrons promote intestinal absorption of lipopolysaccharides. Journal of lipid research, 50, $90-97$.

GNANAPAVAN, S., KOLA, B., BUSTIN, S. A., MORRIS, D. G., MCGEE, P., FAIRCLOUGH, P., BHATTACHARYA, S., CARPENTER, R., GROSSMAN, A. B. \& KORBONITS, M. R. 2002. 
The tissue distribution of the mRNA of ghrelin and subtypes of its receptor, GHS-R, in humans. The Journal of Clinical Endocrinology \& Metabolism, 87, 2988-2988.

GONZALEZ-REY, E., CHORNY, A. \& DELGADO, M. 2006. Therapeutic action of ghrelin in a mouse model of colitis. Gastroenterology, 130, 1707-1720.

GRATTAgliano, I., PAlMieRI, V. O., PORTINCASA, P., MOSChETTA, A. \& PALASCIANO, G. 2008. Oxidative stress-induced risk factors associated with the metabolic syndrome: a unifying hypothesis. The Journal of nutritional biochemistry, 19, 491-504.

GRIFFIN, S. J., LEAVER, J. K. \& IRVING, G. J. 2017. Impact of metformin on cardiovascular disease: a meta-analysis of randomised trials among people with type 2 diabetes. Diabetologia, 60, 1620-1629.

GROSS, J. L., DE AZEVEDO, M. J., SILVEIRO, S. P., CANANI, L. H., CARAMORI, M. L. \& ZELMANOVITZ, T. 2005. Diabetic nephropathy: diagnosis, prevention, and treatment. Diabetes care, 28, 164-176.

GURRIARÁN-RODRÍGUEZ, U., AL-MASSADI, O., ROCA-RIVADA, A., CRUJEIRAS, A. B., GALlEGO, R., PARDO, M., SEOANE, L. M., PAZOS, Y., CASANUEVA, F. F. \& CAMIÑA, J. P. 2011. Obestatin as a regulator of adipocyte metabolism and adipogenesis. Journal of cellular and molecular medicine, 15, 1927-1940.

GUSTAFSON, B. 2010. Adipose tissue, inflammation and atherosclerosis. Journal of atherosclerosis and thrombosis, 17, 332-341.

GUTIERREZ, D. A., PUGLISI, M. J. \& HASTY, A. H. 2009. Impact of increased adipose tissue mass on inflammation, insulin resistance, and dyslipidemia. Current diabetes reports, 9, 26-32.

HARMS, M. \& SEALE, P. 2013. Brown and beige fat: development, function and therapeutic potential. Nature medicine, 19, 1252-1263. 
HARVEY, R. E., HOWARD, V. G., LEMUS, M. B., JOIS, T., ANDREWS, Z. B. \& SLEEMAN, M. W. 2017. The ghrelin/GOAT system regulates obesity-induced inflammation in male mice. Endocrinology.

HATTORI, N. 2009. Expression, regulation and biological actions of growth hormone (GH) and ghrelin in the immune system. Growth Hormone \& IGF Research, 19, 187-197.

HAYASHI, T., KOTANI, H., YAMAGUCHI, T., TAGUCHI, K., IIDA, M., INA, K., MAEDA, M., KUZUYA, M., HATTORI, Y. \& IGNARRO, L. J. 2014. Endothelial cellular senescence is inhibited by liver $\mathrm{X}$ receptor activation with an additional mechanism for its atheroprotection in diabetes. Proceedings of the National Academy of Sciences, 111, 1168-1173.

HIROAKI, U., SHIIYA, T., MIZUTA, M., MONDAL, M. \& NAKAZATO, M. 2007. Plasma ghrelin concentrations in different clinical stages of diabetic complications and glycemic control in Japanese diabetics. Endocrine journal, 54, 895-902.

HOSODA, H., KOJIMA, M., MATSUO, H. \& KANGAWA, K. 2000. Purification and characterization of rat des-GIn14-Ghrelin, a second endogenous ligand for the growth hormone secretagogue receptor. Journal of Biological Chemistry, 275, 2199522000.

HOTAMISLIGIL, G. S., ARNER, P., CARO, J. F., ATKINSON, R. L. \& SPIEGELMAN, B. M. 1995. Increased adipose tissue expression of tumor necrosis factor-alpha in human obesity and insulin resistance. Journal of Clinical Investigation, 95, 2409.

HOTAMISLIGIL, G. S., SHARGILL, N. S. \& SPIEGELMAN, B. M. 1993. Adipose expression of tumor necrosis factor-alpha: direct role in obesity-linked insulin resistance. Science, 259, 87-91. 
HOWELL, G., DENG, X., YelLATURU, C., PARK, E. A., WILCOX, H. G., RAGHOW, R. \& ELAM, M. B. 2009. N-3 polyunsaturated fatty acids suppress insulin-induced SREBP-1C transcription via reduced trans-activating capacity of LXR $\alpha$. Biochimica et Biophysica Acta (BBA)-Molecular and Cell Biology of Lipids, 1791, 1190-1196.

HUDA, M., DOVEY, T., WONG, S., ENGLISH, P., HALFORD, J., MCCULLOCH, P., CLEATOR, J., MARTIN, B., CASHEN, J. \& HAYDEN, K. 2009. Ghrelin restores 'lean-type'hunger and energy expenditure profiles in morbidly obese subjects but has no effect on postgastrectomy subjects. International Journal of Obesity, 33, 317-325.

HYUN, B., SHIN, S., LEE, A., LEE, S., SONG, Y., HA, N.-J., CHO, K.-H. \& KIM, K. 2013. Metformin down-regulates TNF- $\alpha$ secretion via suppression of scavenger receptors in macrophages. Immune network, 13, 123-132.

INTERNATIONAL DIABETES FEDERATION. 2016. IDF Diabetes Atlas, 7th edn. 2016 [Online]. Available: http://www.diabetesatlas.org/ [Accessed 28/11/2016 2016].

INZUCCHI, S. E., BERGENSTAL, R. M., BUSE, J. B., DIAMANT, M., FERRANNINI, E., NAUCK, M., PETERS, A. L., TSAPAS, A., WENDER, R. \& MATTHEWS, D. R. 2015. Management of hyperglycaemia in type 2 diabetes, 2015: a patient-centred approach. Update to a position statement of the American Diabetes Association and the European Association for the Study of Diabetes. Diabetologia, 58, 429-442.

JENSTERLE, M., JANEZ, A., MLINAR, B., MARC, J., PREZEL, J. \& PFEIFER, M. 2008. Impact of metformin and rosiglitazone treatment on glucose transporter 4 mRNA expression in women with polycystic ovary syndrome. European journal of endocrinology, 158, 793-801. 
JING, Y., WU, F., LI, D., YANG, L., LI, Q. \& LI, R. 2017. Metformin improves obesity-associated inflammation by altering macrophages polarization. Molecular and Cellular Endocrinology.

JO, J., GAVRILOVA, O., PACK, S., JOU, W., MULLEN, S., SUMNER, A. E., CUSHMAN, S. W. \& PERIWAL, V. 2009. Hypertrophy and/or hyperplasia: dynamics of adipose tissue growth. PLoS Comput Biol, 5, e1000324.

JOHANSEN, J. S., HARRIS, A. K., RYCHLY, D. J. \& ERGUL, A. 2005. Oxidative stress and the use of antioxidants in diabetes: linking basic science to clinical practice. Cardiovascular diabetology, 4, 1.

KAHN, S. E., ANDRIKOPOULOS, S. \& VERCHERE, C. B. 1999. Islet amyloid: a long-recognized but underappreciated pathological feature of type 2 diabetes. Diabetes, 48, 241-253.

KANG, S., MOON, N. R., KIM, D. S., KIM, S. H. \& PARK, S. 2015. Central acylated ghrelin improves memory function and hippocampal AMPK activation and partly reverses the impairment of energy and glucose metabolism in rats infused with $\beta$-amyloid. Peptides, 71, 84-93.

KATSUKI, A., URAKAWA, H., GABAZZA, E. C., MURASHIMA, S., NAKATANI, K., TOGASHI, K., YANO, Y., ADACHI, Y. \& SUMIDA, Y. 2004. Circulating levels of active ghrelin is associated with abdominal adiposity, hyperinsulinemia and insulin resistance in patients with type 2 diabetes mellitus. European Journal of Endocrinology, 151, 573577.

KEANEY, J. F., LARSON, M. G., VASAN, R. S., WILSON, P. W., LIPINSKA, I., COREY, D., MASSARO, J. M., SUTHERLAND, P., VITA, J. A. \& BENJAMIN, E. J. 2003. Obesity and systemic oxidative stress. Arteriosclerosis, thrombosis, and vascular biology, 23, 434439. 
KELLY, B., TANNAHILL, G. M., MURPHY, M. P. \& O'NEILL, L. A. 2015. Metformin inhibits the production of reactive oxygen species from NADH: ubiquinone oxidoreductase to limit induction of interleukin-1 $\beta$ (IL-1 $\beta)$ and boosts interleukin-10 (IL-10) in lipopolysaccharide (LPS)-activated macrophages. Journal of Biological Chemistry, 290, 20348-20359.

KENNEDY, A., MARTINEZ, K., CHUANG, C.-C., LAPOINT, K. \& MCINTOSH, M. 2009. Saturated fatty acid-mediated inflammation and insulin resistance in adipose tissue: mechanisms of action and implications. The Journal of nutrition, 139, 1-4.

KENNEDY, M. A., BARRERA, G. C., NAKAMURA, K., BALDÁN, Á., TARR, P., FISHBEIN, M. C., FRANK, J., FRANCONE, O. L. \& EDWARDS, P. A. 2005. ABCG1 has a critical role in mediating cholesterol efflux to $\mathrm{HDL}$ and preventing cellular lipid accumulation. Cell metabolism, 1, 121-131.

KERSTEN, S. 2001. Mechanisms of nutritional and hormonal regulation of lipogenesis. EMBO reports, 2, 282-286.

KIM, J. B. \& SPIEGELMAN, B. M. 1996. ADD1/SREBP1 promotes adipocyte differentiation and gene expression linked to fatty acid metabolism. Genes \& development, 10, 10961107.

KIM, S.-P., HA, J. M., YUN, S. J., KIM, E. K., CHUNG, S. W., HONG, K. W., KIM, C. D. \& BAE, S. S. 2010. Transcriptional activation of peroxisome proliferator-activated receptor- $\psi$ requires activation of both protein kinase $A$ and Akt during adipocyte differentiation. Biochemical and biophysical research communications, 399, 55-59.

KIRCHNER, H., HEPPNER, K. M. \& TSCHÖP, M. H. 2012. The role of ghrelin in the control of energy balance. Appetite Control. Springer. 
KISHORE, V., BULLOCK, W., SUN, X., VAN DYKE, W. S. \& AKKUS, O. 2012. Tenogenic differentiation of human MSCs induced by the topography of electrochemically aligned collagen threads. Biomaterials, 33, 2137-2144.

KNEBEL, B., HAAS, J., HARTWIG, S., JACOB, S., KÖLLMER, C., NITZGEN, U., MULLER-WIELAND, D. \& KOTZKA, J. 2012. Liver-specific expression of transcriptionally active SREBP-1c is associated with fatty liver and increased visceral fat mass. PloS one, 7, e31812.

KOHNO, D., SONE, H., MINOKOSHI, Y. \& YADA, T. 2008. Ghrelin raises [Ca2+] i via AMPK in hypothalamic arcuate nucleus NPY neurons. Biochemical and biophysical research communications, 366, 388-392.

KOISTINEN, H., BASTARD, J., DUSSERRE, E., EBELING, P., ZEGARI, N., ANDREELLI, F., JARDEL, C., DONNER, M., MEYER, L. \& MOULIN, P. 2000. Subcutaneous adipose tissue expression of tumour necrosis factor-a is not associated with whole body insulin resistance in obese nondiabetic or in type-2 diabetic subjects. European journal of clinical investigation, 30, 302-310.

KOJIMA, M. 2008. The discovery of ghrelin-a personal memory. Regulatory peptides, 145, 2-6.

KOJIMA, M., HOSODA, H., DATE, Y., NAKAZATO, M., MATSUO, H. \& KANGAWA, K. 1999. Ghrelin is a growth-hormone-releasing acylated peptide from stomach. Nature, 402, 656-660.

KOJIMA, M. \& KANGAWA, K. 2005. Ghrelin: structure and function. Physiological reviews, $85,495-522$.

KOJIMA, M. \& KANGAWA, K. 2008. Structure and function of ghrelin. Orphan G ProteinCoupled Receptors and Novel Neuropeptides. Springer. 
KOLA, B., hUbINA, E., TUCCI, S. A., KIRKHAM, T. C., GARCIA, E. A., MITCHELL, S. E., WILliAMS, L. M., HAWLEY, S. A., HARDIE, D. G. \& GROSSMAN, A. B. 2005. Cannabinoids and ghrelin have both central and peripheral metabolic and cardiac effects via AMP-activated protein kinase. Journal of Biological Chemistry, 280, 25196-25201.

KORBONITS, M., GOLDSTONE, A. P., GUEORGUIEV, M. \& GROSSMAN, A. B. 2004. Ghrelin-a hormone with multiple functions. Frontiers in neuroendocrinology, 25, 27-68.

KWON, H. \& PESSIN, J. E. 2013. Adipokines mediate inflammation and insulin resistance. Frontiers in endocrinology, 4.

LAM, T. K., VAN DE WERVE, G. \& GIACCA, A. 2003. Free fatty acids increase basal hepatic glucose production and induce hepatic insulin resistance at different sites. American Journal of Physiology-Endocrinology And Metabolism, 284, E281-E290.

LAM, Y. Y., MITCHELL, A. J., HOLMES, A. J., DENYER, G. S., GUMMESSON, A., CATERSON, I. D., HUNT, N. H. \& STORLIEN, L. H. 2011. Role of the gut in visceral fat inflammation and metabolic disorders. Obesity, 19, 2113-2120.

LAPPAS, M. 2014. Effect of pre-existing maternal obesity, gestational diabetes and adipokines on the expression of genes involved in lipid metabolism in adipose tissue. Metabolism, 63, 250-262.

LE LAY, S., KRIEF, S., FARNIER, C., LEFRÈRE, I., LE LIEPVRE, X., BAZIN, R., FERRÉ, P. \& DUGAIL, I. 2001. Cholesterol, a cell size-dependent signal that regulates glucose metabolism and gene expression in adipocytes. Journal of Biological Chemistry, 276, 1690416910.

LI, D., WANG, D., WANG, Y., LING, W., FENG, X. \& XIA, M. 2010a. Adenosine monophosphate-activated protein kinase induces cholesterol efflux from 
macrophage-derived foam cells and alleviates atherosclerosis in apolipoprotein Edeficient mice. Journal of Biological Chemistry, 285, 33499-33509.

LI, D., ZHANG, Y., MA, J., LING, W. \& XIA, M. 2010b. Adenosine monophosphate activated protein kinase regulates $A B C G 1$-mediated oxysterol efflux from endothelial cells and protects against hypercholesterolemia-induced endothelial dysfunction. Arteriosclerosis, thrombosis, and vascular biology, 30, 1354-1362.

LIHN, A. S., PEDERSEN, S. B., LUND, S. \& RICHELSEN, B. 2008. The anti-diabetic AMPK activator AICAR reduces IL-6 and IL-8 in human adipose tissue and skeletal muscle cells. Molecular and cellular endocrinology, 292, 36-41.

LIM, C. T., KOLA, B., GROSSMAN, A. \& KORBONITS, M. 2011. The expression of ghrelin Oacyltransferase (GOAT) in human tissues. Endocrine journal, 58, 707-710.

LIN, H. Z., YANG, S. Q., CHUCKAREE, C., KUHAJDA, F., RONNET, G. \& DIEHL, A. M. 2000. Metformin reverses fatty liver disease in obese, leptin-deficient mice. Nature medicine, 6, 998.

LIN, L., LEE, J. H., BURAS, E. D., YU, K., WANG, R., SMITH, C. W., WU, H., SHEIKH-HAMAD, D. \& SUN, Y. 2016. Ghrelin receptor regulates adipose tissue inflammation in aging. Aging (Albany NY), 8, 178.

LIU, S., LI, X., LI, Z., HE, L., XIAO, Y., YAN, K. \& ZHOU, Z. 2012. Octanoylated Ghrelin Inhibits the Activation of the Palmitic Acid-Induced TLR4/NF-B Signaling Pathway in THP-1 Macrophages. ISRN endocrinology, 2012.

LIVAK, K. J. \& SCHMITTGEN, T. D. 2001. Analysis of relative gene expression data using realtime quantitative PCR and the 2- $\Delta \Delta C T$ method. methods, 25, 402-408.

LÖFGREN, P., VAN HARMELEN, V., REYNISDOTTIR, S., NÄSLUND, E., RYDÉN, M., RÖSSNER, S. \& ARNER, P. 2000. Secretion of tumor necrosis factor-alpha shows a strong 
relationship to insulin-stimulated glucose transport in human adipose tissue. Diabetes, 49, 688-692.

LUO, F., GUO, Y., RUAN, G.-Y., LONG, J.-K., ZHENG, X.-L., XIA, Q., ZHAO, S.-P., PENG, D.-Q., FANG, Z.-F. \& LI, X.-P. 2017. Combined use of metformin and atorvastatin attenuates atherosclerosis in rabbits fed a high-cholesterol diet. Scientific reports, 7, 2169.

LUO, F., GUO, Y., RUAN, G. \& LI, X. 2016. Metformin promotes cholesterol efflux in macrophages by up-regulating FGF21 expression: a novel anti-atherosclerotic mechanism. Lipids in health and disease, 15, 109.

MA, A. Z., SONG, Z. Y. \& ZHANG, Q. 2014. Cholesterol efflux is LXRalpha isoform-dependent in human Macrophages. BMC cardiovascular disorders, 14, 80.

MAACHI, M., PIERONI, L., BRUCKERT, E., JARDEL, C., FELLAHI, S., HAINQUE, B., CAPEAU, J. \& BASTARD, J. 2004. Systemic low-grade inflammation is related to both circulating and adipose tissue TNF $\alpha$, leptin and IL-6 levels in obese women. International journal of obesity, 28, 993-997.

MADSEN-BOUTERSE, S. A. \& KOWLURU, R. A. 2008. Oxidative stress and diabetic retinopathy: pathophysiological mechanisms and treatment perspectives. Reviews in Endocrine and Metabolic Disorders, 9, 315-327.

MAKKI, K., FROGUEL, P. \& WOLOWCZUK, I. 2013. Adipose tissue in obesity-related inflammation and insulin resistance: cells, cytokines, and chemokines. ISRN inflammation, 2013.

MAQDASY, S., TROUSSON, A., TAUVERON, I., VOLLE, D. H., BARON, S. \& LOBACCARO, J.-M. A. 2016. Once and for all, LXR $\alpha$ and LXR $\beta$ are gatekeepers of the endocrine system. Molecular aspects of medicine, 49, 31-46. 
MÅRIN, P., ANDERSSON, B., OTTOSSON, M., OLBE, L., CHOWDHURY, B., KVIST, H., HOLM, G., SJÖSTRÖM, L. \& BJÖRNTORP, P. 1992. The morphology and metabolism of intraabdominal adipose tissue in men. Metabolism, 41, 1242-1248.

MARQUES-NEVES, C. 2015. Diabetic retinopathy-pathophysiology. Acta Ophthalmologica, 93.

MARTÍN-GALLÁN, P., CARRASCOSA, A., GUSSINYÉ, M. \& DOMÍNGUEZ, C. 2003. Biomarkers of diabetes-associated oxidative stress and antioxidant status in young diabetic patients with or without subclinical complications. Free Radical Biology and Medicine, 34, 1563-1574.

MARTÍN-TIMÓN, I., SEVILLANO-COLLANTES, C., SEGURA-GALINDO, A. \& DEL CAÑIZOGÓMEZ, F. J. 2014. Type 2 diabetes and cardiovascular disease: have all risk factors the same strength? World journal of diabetes, 5, 444.

MASMIQUEL, L., LEITER, L., VIDAL, J., BAIN, S., PETRIE, J., FRANEK, E., RAZ, I., COMLEKCI, A., JACOB, S. \& GAAL, L. V. 2016. LEADER 5: prevalence and cardiometabolic impact of obesity in cardiovascular high-risk patients with type 2 diabetes mellitus: baseline global data from the LEADER trial. Cardiovascular diabetology, 15, 29.

MASUDA, Y., TANAKA, T., INOMATA, N., OHNUMA, N., TANAKA, S., ITOH, Z., HOSODA, H., KOJIMA, M. \& KANGAWA, K. 2000. Ghrelin stimulates gastric acid secretion and motility in rats. Biochemical and biophysical research communications, 276, 905-908.

MATSUDA, M. \& SHIMOMURA, I. 2013. Increased oxidative stress in obesity: implications for metabolic syndrome, diabetes, hypertension, dyslipidemia, atherosclerosis, and cancer. Obesity research \& clinical practice, 7, e330-e341.

MATSUZAWA, Y. 2006. Therapy insight: adipocytokines in metabolic syndrome and related cardiovascular disease. Nature clinical practice Cardiovascular medicine, 3, 35-42. 
MATSUZAWA, Y., FUNAHASHI, T. \& NAKAMURA, T. 1999. Molecular Mechanism of Metabolic Syndrome X: Contribution of Adipocytokines- Adipocyte-derived Bioactive Substances. Annals of the New York Academy of Sciences, 892, 146-154.

MAUERER, R., EBERT, S. \& LANGMANN, T. 2009. High glucose, unsaturated and saturated fatty acids differentially regulate expression of ATP-binding cassette transporters $A B C A 1$ and $A B C G 1$ in human macrophages. Experimental \& molecular medicine, 41, 126.

MAUMUS, M., SENGENES, C., DECAUNES, P., ZAKAROFF-GIRARD, A., BOURLIER, V., LAFONTAN, M., GALITZKY, J. \& BOULOUMIÉ, A. 2008. Evidence of in situ proliferation of adult adipose tissue-derived progenitor cells: influence of fat mass microenvironment and growth. The Journal of Clinical Endocrinology \& Metabolism, 93, 4098-4106.

MAURY, E., EHALA-ALEKSEJEV, K., GUIOT, Y., DETRY, R., VANDENHOOFT, A. \& BRICHARD, S. M. 2007. Adipokines oversecreted by omental adipose tissue in human obesity. American Journal of Physiology-Endocrinology and Metabolism, 293, E656-E665.

MCCREIGHT, L. J., BAILEY, C. J. \& PEARSON, E. R. 2016. Metformin and the gastrointestinal tract. Diabetologia, 59, 426-435.

MCLAUGHLIN, T., ACKERMAN, S. E., SHEN, L. \& ENGLEMAN, E. 2017. Role of innate and adaptive immunity in obesity-associated metabolic disease. The Journal of clinical investigation, 127, 5-13.

MEHLEM, A., HAGBERG, C. E., MUHL, L., ERIKSSON, U. \& FALKEVALL, A. 2013. Imaging of neutral lipids by oil red $\mathrm{O}$ for analyzing the metabolic status in health and disease. Nature protocols, 8, 1149-1154. 
MEIER, U. \& GRESSNER, A. M. 2004. Endocrine regulation of energy metabolism: review of pathobiochemical and clinical chemical aspects of leptin, ghrelin, adiponectin, and resistin. Clinical Chemistry, 50, 1511-1525.

MIEGUEU, P., ST PIERRE, D., BROGLIO, F. \& CIANFLONE, K. 2011. Effect of desacyl ghrelin, obestatin and related peptides on triglyceride storage, metabolism and GHSR signaling in 3T3-L1 adipocytes. Journal of cellular biochemistry, 112, 704-714.

MISHRA, A. K., DUBEY, V. \& GHOSH, A. R. 2016. Obesity: An overview of possible role (s) of gut hormones, lipid sensing and gut microbiota. Metabolism, 65, 48-65.

MITRO, N., MAK, P. A., VARGAS, L., GODIO, C., HAMPTON, E., MOLTENI, V., KREUSCH, A. \& SAEZ, E. 2007. The nuclear receptor LXR is a glucose sensor. Nature, 445, 219.

MORENO-NAVARRETE, J. M. \& FERNÁNDEZ-REAL, J. M. 2012. Adipocyte differentiation. Adipose tissue biology. Springer.

MORENO-NAVARRETE, J. M., ORTEGA, F. J., RODRÍGUEZ-HERMOSA, J.-I., SABATER, M., PARDO, G., RICART, W. \& FERNÁNDEZ-REAL, J. M. 2011. OCT1 expression in adipocytes could contribute to increased metformin action in obese subjects. Diabetes, 60, 168-176.

MUCCIOLI, G., PONS, N., GHÈ, C., CATAPANO, F., GRANATA, R. \& GHIGO, E. 2004. Ghrelin and des-acyl ghrelin both inhibit isoproterenol-induced lipolysis in rat adipocytes via a non-type 1a growth hormone secretagogue receptor. European journal of pharmacology, 498, 27-35.

MÜLLER, T., NOGUEIRAS, R., ANDERMANN, M., ANDREWS, Z., ANKER, S., ARGENTE, J., BATTERHAM, R., BENOIT, S., BOWERS, C. \& BROGLIO, F. 2015. Ghrelin. Molecular Metabolism, 4, 437-460. 
NATIONAL INSTITUTE HEALTH AND CARE EXCELLENCE. 2015. Type 2 diabetes in adults: management. (NICE guideline 28.) [Online]. Available:
(N) https://www.nice.org.uk/guidance/ng28 [Accessed 15th January 2018].

NINOMIYA, Y., SUGAHARA-YAMASHITA, Y., NAKACHI, Y., TOKUZAWA, Y., OKAZAKI, Y. \& NISHIYAMA, M. 2010. Development of a rapid culture method to induce adipocyte differentiation of human bone marrow-derived mesenchymal stem cells. Biochemical and biophysical research communications, 394, 303-308.

O'KENNEDY, R., BYRNE, M., O'FAGAIN, C. \& BERNS, G. 1990. Experimental section: a review of enzyme-immunoassay and a description of a competitive enzyme-linked immunosorbent assay for the detection of immunoglobulin concentrations. Biochemical Education, 18, 136-140.

ORAVA, J., NUUTILA, P., LIDELL, M. E., OIKONEN, V., NOPONEN, T., VILJANEN, T., SCHEININ, M., TAITTONEN, M., NIEMI, T. \& ENERBÄCK, S. 2011. Different metabolic responses of human brown adipose tissue to activation by cold and insulin. Cell metabolism, 14, 272-279.

OUCHI, N., PARKER, J. L., LUGUS, J. J. \& WALSH, K. 2011. Adipokines in inflammation and metabolic disease. Nature Reviews Immunology, 11, 85-97.

PEDRAM, A., RAZANDI, M., O'MAHONY, F., HARVEY, H., HARVEY, B. J. \& LEVIN, E. R. 2013. Estrogen reduces lipid content in the liver exclusively from membrane receptor signaling. Sci. Signal., 6, ra36-ra36.

PEINO, R., BALDELLI, R., RODRIGUEZ-GARCIA, J., RODRIGUEZ-SEGADE, S., KOJIMA, M., KANGAWA, K., ARVAT, E., GHIGO, E., DIEGUEZ, C. \& CASANUEVA, F. F. 2000. Ghrelininduced growth hormone secretion in humans. European Journal of Endocrinology, 143, R11-R14. 
PEREIRA, J. A. D. S., SILVA, F. C. D. \& DE MORAES-VIEIRA, P. M. M. 2017. The Impact of Ghrelin in Metabolic Diseases: An Immune Perspective. Journal of diabetes research, 2017.

PHOENIX, K. N., VUMBACA, F. \& CLAFFEY, K. P. 2009. Therapeutic metformin/AMPK activation promotes the angiogenic phenotype in the ER $\alpha$ negative MDA-MB-435 breast cancer model. Breast cancer research and treatment, 113, 101-111.

PLUM, L., BELGARDT, B. F. \& BRÜNING, J. C. 2006. Central insulin action in energy and glucose homeostasis. The Journal of clinical investigation, 116, 1761-1766.

POPA, S. \& MOTA, M. 2013. Beta-Cell Function and Failure in Type 2 Diabetes. Type, 2, 2950.

PORTEIRO, B., DÍAZ-RUÍZ, A., MARTíNEZ, G., SENRA, A., VIDAL, A., SERRANO, M., GUALILlO, O., LÓPEZ, M., MALAGÓN, M. M. \& DIÉGUEZ, C. 2013. Ghrelin requires p53 to stimulate lipid storage in fat and liver. Endocrinology, 154, 3671-3679.

PÖYKKÖ, S. M., KELLOKOSKI, E., HÖRKKÖ, S., KAUMA, H., KESÄNIEMI, Y. A. \& UKKOLA, O. 2003. Low plasma ghrelin is associated with insulin resistance, hypertension, and the prevalence of type 2 diabetes. Diabetes, 52, 2546-2553.

PRIOR, S. L., BARRY, J. D., CAPLIN, S., MIN, T., GRANT, D. A. \& STEPHENS, J. W. 2017. Temporal changes in plasma markers of oxidative stress following laparoscopic sleeve gastrectomy in subjects with impaired glucose regulation. Surgery for Obesity and Related Diseases, 13, 162-168.

QARNI, A., AHMED, A., JOATAR, F. E., DAS, N., AWAD, M., ElTAYEB, M., AL-ZUBAIR, A. G., ALI, M. E., AL MASAUD, A. \& SHIRE, A. M. 2017. Association of plasma ghrelin levels with insulin resistance in type 2 diabetes mellitus among Saudi subjects. Endocrinology and Metabolism, 32, 230-240. 
QIU, Z., WEI, Y., CHEN, N., JIANG, M., WU, J. \& LIAO, K. 2001. DNA synthesis and mitotic clonal expansion is not a required step for 3T3-L1 preadipocyte differentiation into adipocytes. Journal of Biological Chemistry, 276, 11988-11995.

RENA, G., HARDIE, D. G. \& PEARSON, E. R. 2017. The mechanisms of action of metformin. Diabetologia, 60, 1577-1585.

REPA, J. J., LIANG, G., OU, J., BASHMAKOV, Y., LOBACCARO, J.-M. A., SHIMOMURA, I., SHAN, B., BROWN, M. S., GOLDSTEIN, J. L. \& MANGELSDORF, D. J. 2000. Regulation of mouse sterol regulatory element-binding protein-1c gene (SREBP-1c) by oxysterol receptors, LXR $\alpha$ and LXRß. Genes \& development, 14, 2819-2830.

REPETTO, M., BOVERIS, A. \& SEMPRINE, J. 2012. Lipid peroxidation: chemical mechanism, biological implications and analytical determination, INTECH Open Access Publisher.

ROBERTSON, R. P., HARMON, J., TRAN, P. O., TANAKA, Y. \& TAKAHASHI, H. 2003. Glucose toxicity in $\beta$-cells: type 2 diabetes, good radicals gone bad, and the glutathione connection. Diabetes, 52, 581-587.

RODRIGUEZ, A., GOMEZ-AMBROSI, J., CATALAN, V., GIL, M., BECERRIL, S., SAINZ, N., SILVA, C., SALVADOR, J., COLINA, I. \& FRÜHBECK, G. 2009. Acylated and desacyl ghrelin stimulate lipid accumulation in human visceral adipocytes. International journal of obesity, 33, 541-552.

RODRÍGUEZ, A., GÓMEZ-AMBROSI, J., CATALÁN, V., ROTELLAR, F., VALENTI, V., SILVA, C., MuguetA, C., PULIDO, M., VÁZqUEZ, R. \& SALVADOR, J. 2012. The ghrelin Oacyltransferase-ghrelin system reduces TNF- $\alpha$-induced apoptosis and autophagy in human visceral adipocytes. Diabetologia, 55, 3038-3050.

RONTI, T., LUPATTELLI, G. \& MANNARINO, E. 2006. The endocrine function of adipose tissue: an update. Clinical endocrinology, 64, 355-365. 
ROSEN, E. D. \& SPIEGELMAN, B. M. 2014. What we talk about when we talk about fat. Cell, $156,20-44$.

ROTTER, V., NAGAEV, I. \& SMITH, U. 2003. Interleukin-6 (IL-6) induces insulin resistance in 3T3-L1 adipocytes and is, like IL-8 and tumor necrosis factor- $\alpha$, overexpressed in human fat cells from insulin-resistant subjects. Journal of Biological Chemistry, 278, 45777-45784.

SAGHIZADEH, M., ONG, J. M., GARVEY, W. T., HENRY, R. R. \& KERN, P. A. 1996. The expression of TNF alpha by human muscle. Relationship to insulin resistance. Journal of Clinical Investigation, 97, 1111.

SALASZNYK, R. M., KLEES, R. F., WESTCOTT, A. M., VANDENBERG, S., BENNETT, K. \& PLOPPER, G. E. 2005. Focusing of gene expression as the basis of stem cell differentiation. Stem cells and development, 14, 608-620.

SANCHEZ-RANGEL, E. \& INZUCCHI, S. E. 2017. Metformin: clinical use in type 2 diabetes. Diabetologia, 60, 1586-1593.

SATO, T., NAKAMURA, Y., SHIIMURA, Y., OHGUSU, H., KANGAWA, K. \& KOJIMA, M. 2012. Structure, regulation and function of ghrelin. Journal of biochemistry, 151, 119-128.

SATOU, M., NISHI, Y., YOH, J., HATTORI, Y. \& SUGIMOTO, H. 2010. Identification and characterization of acyl-protein thioesterase 1/lysophospholipase I as a ghrelin deacylation/lysophospholipid hydrolyzing enzyme in fetal bovine serum and conditioned medium. Endocrinology, 151, 4765-4775.

SCERIF, M., GOLDSTONE, A. P. \& KORBONITS, M. 2011. Ghrelin in obesity and endocrine diseases. Molecular and cellular endocrinology, 340, 15-25.

SCHMITTGEN, T. D. \& LIVAK, K. J. 2008. Analyzing real-time PCR data by the comparative CT method. Nature protocols, 3, 1101-1108. 
SCHULTZ, J. R., TU, H., LUK, A., REPA, J. J., MEDINA, J. C., LI, L., SCHWENDNER, S., WANG, S., THOOLEN, M. \& MANGELSDORF, D. J. 2000. Role of LXRs in control of lipogenesis. Genes \& development, 14, 2831-2838.

SCOTT, M. A., NGUYEN, V. T., LEVI, B. \& JAMES, A. W. 2011. Current methods of adipogenic differentiation of mesenchymal stem cells. Stem cells and development, 20, 17931804.

SENN, J. J., KLOVER, P. J., NOWAK, I. A., ZIMMERS, T. A., KONIARIS, L. G., FURLANETTO, R. W. \& MOONEY, R. A. 2003. Suppressor of cytokine signaling-3 (SOCS-3), a potential mediator of interleukin-6-dependent insulin resistance in hepatocytes. Journal of Biological Chemistry, 278, 13740-13746.

SERBY, M. D., ZHAO, H., SZCZEPANKIEWICZ, B. G., KOSOGOF, C., XIN, Z., LIU, B., LIU, M., NELSON, L. T., KASZUBSKA, W. \& FALLS, H. D. 2006. 2, 4-diaminopyrimidine derivatives as potent growth hormone secretagogue receptor antagonists. Journal of medicinal chemistry, 49, 2568-2578.

SHANADO, Y., KOMETANI, M., UCHIYAMA, H., KOIZUMI, S. \& TENO, N. 2004. Lysophospholipase I identified as a ghrelin deacylation enzyme in rat stomach. Biochemical and biophysical research communications, 325, 1487-1494.

SHEN, W., WANG, Z., PUNYANITA, M., LEI, J., SINAV, A., KRAL, J. G., IMIELINSKA, C., ROSS, R. \& HEYMSFIELD, S. B. 2003. Adipose tissue quantification by imaging methods: a proposed classification. Obesity, 11, 5-16.

SHI, H., KOKOEVA, M. V., INOUYE, K., TZAMELI, I., YIN, H. \& FLIER, J. S. 2006. TLR4 links innate immunity and fatty acid-induced insulin resistance. Journal of Clinical Investigation, 116, 3015. 
SHIIYA, T., NAKAZATO, M., MIZUTA, M., DATE, Y., MONDAL, M. S., TANAKA, M., NOZOE, S.-I., HOSODA, H., KANGAWA, K. \& MATSUKURA, S. 2002. Plasma ghrelin levels in lean and obese humans and the effect of glucose on ghrelin secretion. The Journal of Clinical Endocrinology \& Metabolism, 87, 240-244.

SHIMADA, T., FURUTA, H., DOI, A., ARIYASU, H., KAWASHIMA, H., WAKASAKI, H., NISHI, M., SASAKI, H. \& AKAMIZU, T. 2014. Des-acyl ghrelin protects microvascular endothelial cells from oxidative stress-induced apoptosis through sirtuin 1 signaling pathway. Metabolism, 63, 469-474.

SHOELSON, S. E., HERRERO, L. \& NAAZ, A. 2007. Obesity, inflammation, and insulin resistance. Gastroenterology, 132, 2169-2180.

SKURK, T., ALBERTI-HUBER, C., HERDER, C. \& HAUNER, H. 2007. Relationship between adipocyte size and adipokine expression and secretion. The Journal of Clinical Endocrinology \& Metabolism, 92, 1023-1033.

SPIEGELMAN, B. M. \& FLIER, J. S. 1996. Adipogenesis and obesity: rounding out the big picture. Cell, 87, 377-389.

SPIEGELMAN, B. M. \& FLIER, J. S. 2001. Obesity and the regulation of energy balance. Cell, 104, 531-543.

STANFORD, K. I., MIDDELBEEK, R. J., TOWNSEND, K. L., AN, D., NYGAARD, E. B., HITCHCOX, K. M., MARKAN, K. R., NAKANO, K., HIRSHMAN, M. F. \& TSENG, Y.-H. 2013. Brown adipose tissue regulates glucose homeostasis and insulin sensitivity. The Journal of clinical investigation, 123, 215-223.

STANLEY, T. L., ZANNI, M. V., JOHNSEN, S., RASHEED, S., MAKIMURA, H., LEE, H., KHOR, V. K., AHIMA, R. S. \& GRINSPOON, S. K. 2011. TNF- $\alpha$ antagonism with etanercept decreases glucose and increases the proportion of high molecular weight 
adiponectin in obese subjects with features of the metabolic syndrome. The Journal of Clinical Endocrinology \& Metabolism, 96, E146-E150.

STENVINKEL, P., KETTELER, M., JOHNSON, R. J., LINDHOLM, B., PECOITS-FILHO, R., RIELLA, M., HEIMBÜRGER, O., CEDERHOLM, T. \& GIRNDT, M. 2005. IL-10, IL-6, and TNF- $\alpha$ : central factors in the altered cytokine network of uremia - the good, the bad, and the ugly. Kidney international, 67, 1216-1233.

STEPHENS, J. W., KHANOLKAR, M. P. \& BAIN, S. C. 2009. The biological relevance and measurement of plasma markers of oxidative stress in diabetes and cardiovascular disease. Atherosclerosis, 202, 321-329.

STERNE, J. 1957. Blood sugar-lowering effect of 1, 1-dimethylbiguanide. Therapie, 13, 650659.

STOCKER, D. J., TAYLOR, A. J., LANGLEY, R. W., JEZIOR, M. R. \& VIGERSKY, R. A. 2007. A randomized trial of the effects of rosiglitazone and metformin on inflammation and subclinical atherosclerosis in patients with type 2 diabetes. American heart journal, 153, 445. e1-445. e6.

STRATTON, I. M., ADLER, A. I., NEIL, H. A. W., MATTHEWS, D. R., MANLEY, S. E., CULL, C. A., HADDEN, D., TURNER, R. C. \& HOLMAN, R. R. 2000. Association of glycaemia with macrovascular and microvascular complications of type 2 diabetes (UKPDS 35): prospective observational study. Bmj, 321, 405-412.

SUEMATSU, M., KATSUKI, A., SUMIDA, Y., GABAZZA, E. C., MURASHIMA, S., MATSUMOTO, K., KITAGAWA, N., AKATSUKA, H., HORI, Y. \& NAKATANI, K. 2005. Decreased circulating levels of active ghrelin are associated with increased oxidative stress in obese subjects. European journal of endocrinology, 153, 403-407. 
SUPÁK, D., MELCZER, Z. \& CSEH, K. 2016. Elevated serum acylated (biologically active) ghrelin and resistin levels associate with pregnancy-induced weight gain, insulin resistance and antropometric data in the fetus. European Journal of Obstetrics and Gynecology and Reproductive Biology, 206, e111.

TANGVARASITTICHAI, S. 2015. Oxidative stress, insulin resistance, dyslipidemia and type 2 diabetes mellitus. World J Diabetes, 6, 456-480.

TIEDGE, M., LORTZ, S., DRINKGERN, J. \& LENZEN, S. 1997. Relation between antioxidant enzyme gene expression and antioxidative defense status of insulin-producing cells. Diabetes, 46, 1733-1742.

TOKUNAGA, T., HUME, W. E., NAGAMINE, J., KAWAMURA, T., TAIJI, M. \& NAGATA, R. 2005. Structure-activity relationships of the oxindole growth hormone secretagogues. Bioorganic \& medicinal chemistry letters, 15, 1789-1792.

TOZZO, E., GNUDI, L. \& KAHN, B. B. 1997. Amelioration of insulin resistance in streptozotocin diabetic mice by transgenic overexpression of GLUT4 driven by an adipose-specific promoter. Endocrinology, 138, 1604-1611.

TRAYHURN, P. \& WOOD, I. S. 2004. Adipokines: inflammation and the pleiotropic role of white adipose tissue. British Journal of Nutrition, 92, 347-355.

TRUJILLO, M. E. \& SCHERER, P. E. 2006. Adipose tissue-derived factors: impact on health and disease. Endocrine reviews, 27, 762-778.

TSUBONE, T., MASAKI, T., KATSURAGI, I., TANAKA, K., KAKUMA, T. \& YOSHIMATSU, H. 2005. Ghrelin regulates adiposity in white adipose tissue and UCP1 mRNA expression in brown adipose tissue in mice. Regulatory peptides, 130, 97-103. 
VALKO, M., LEIBFRITZ, D., MONCOL, J., CRONIN, M. T., MAZUR, M. \& TELSER, J. 2007. Free radicals and antioxidants in normal physiological functions and human disease. The international journal of biochemistry \& cell biology, 39, 44-84.

VAN EXEL, E., GUSSEKLOO, J., DE CRAEN, A. J., FRÖLICH, M., BOOTSMA-VAN DER WIEL, A. \& WESTENDORP, R. G. 2002. Low production capacity of interleukin-10 associates with the metabolic syndrome and type 2 diabetes. Diabetes, 51, 1088-1092.

VAN MARKEN LICHTENBELT, W. D., VANHOMMERIG, J. W., SMULDERS, N. M., DROSSAERTS, J. M., KEMERINK, G. J., BOUVY, N. D., SCHRAUWEN, P. \& TEULE, G. J. 2009. Coldactivated brown adipose tissue in healthy men. New England Journal of Medicine, $360,1500-1508$.

VAUGHAN, A. M. \& ORAM, J. F. 2005. ABCG1 redistributes cell cholesterol to domains removable by high density lipoprotein but not by lipid-depleted apolipoproteins. Journal of Biological Chemistry, 280, 30150-30157.

VELÁSQUEZ, D. A., MARTínEZ, G., ROMERO, A., VÁZQUEZ, M. J., BOIT, K. D., DOPESO-REYES, I. G., LÓPEZ, M., VIDAL, A., NOGUEIRAS, R. \& DIÉGUEZ, C. 2011. The central Sirtuin 1/p53 pathway is essential for the orexigenic action of ghrelin. Diabetes, $60,1177-$ 1185.

VestergaARD, E. T., BUHL, M., GJedSTED, J., MADSEN, M., JeSSEN, N., NIELSEN, S., GAYLINN, B. D., LIU, J., THORNER, M. O. \& MOLLER, N. 2010. Acute peripheral metabolic effects of intraarterial ghrelin infusion in healthy young men. The Journal of Clinical Endocrinology \& Metabolism, 96, 468-477.

VestergaARD, E. T., hanSEN, T. K., GORMSEN, L. C., JAKOBSEN, P., MOlleR, N., CHRISTIANSEN, J. S. \& JORGENSEN, J. O. L. 2007. Constant intravenous ghrelin 
infusion in healthy young men: clinical pharmacokinetics and metabolic effects. American Journal of Physiology-Endocrinology and Metabolism, 292, E1829-E1836.

VIRTANEN, K. A., LIDELL, M. E., ORAVA, J., HEGLIND, M., WeSteRgReN, R., NIEMI, T., TAITTONEN, M., LAINE, J., SAVISTO, N.-J. \& ENERBÄCK, S. 2009. Functional brown adipose tissue in healthy adults. New England Journal of Medicine, 360, 1518-1525.

WABITSCH, M., BRENNER, R., MELZNER, I., BRAUN, M., MÖLLER, P., HEINZE, E., DEBATIN, K. \& HAUNER, H. 2001. Characterization of a human preadipocyte cell strain with high capacity for adipose differentiation. International journal of obesity, 25, 8.

WAGNER, B. L., VALLEDOR, A. F., SHAO, G., DAIGE, C. L., BISCHOFF, E. D., PETROWSKI, M., JEPSEN, K., BAEK, S. H., HEYMAN, R. A. \& ROSENFELD, M. G. 2003. Promoter-specific roles for liver $\mathrm{X}$ receptor/corepressor complexes in the regulation of $\mathrm{ABCA} 1$ and SREBP1 gene expression. Molecular and cellular biology, 23, 5780-5789.

WANG, G., LEE, H.-M., ENGLANDER, E. \& GREELEY, G. H. 2002. Ghrelin-not just another stomach hormone. Regulatory peptides, 105, 75-81.

WANG, J., ZHU, L., HU, K., TANG, Y., ZENG, X., LIU, J. \& XU, J. 2017. Effects of metformin treatment on serum levels of C-reactive protein and interleukin-6 in women with polycystic ovary syndrome: a meta-analysis: A PRISMA-compliant article. Medicine, 96.

WASEEM, T., DUXBURY, M., ITO, H., ASHLEY, S. W. \& ROBINSON, M. K. 2008. Exogenous ghrelin modulates release of pro-inflammatory and anti-inflammatory cytokines in LPS-stimulated macrophages through distinct signaling pathways. Surgery, 143, 334342.

WASS, J. \& OWEN, K. 2014. Oxford handbook of endocrinology and diabetes, OUP Oxford. WELLS, T. 2009. Ghrelin-defender of fat. Progress in lipid research, 48, 257-274. 
WINDER, W. \& HARDIE, D. 1999. AMP-activated protein kinase, a metabolic master switch: possible roles in type 2 diabetes. American Journal of Physiology-Endocrinology And Metabolism, 277, E1-E10.

WISSE, B. E. 2004. The inflammatory syndrome: the role of adipose tissue cytokines in metabolic disorders linked to obesity. Journal of the American society of nephrology, $15,2792-2800$.

WOOD, S. I. \& TRAYHURN, P. 2006. Adipokines and the signaling role of adipose tissue in inflammation and obesity. Future Lipidology, 1, 81-89.

WORLD HEALTH ORGANIZATION. 2016. FACTSHEET: Obesity and overweight [Online]. http://www.who.int/mediacentre/factsheets/fs311/en/. Available: http://www.who.int/mediacentre/factsheets/fs311/en/ [Accessed 19 September 2016].

WU, J., COHEN, P. \& SPIEGELMAN, B. M. 2013. Adaptive thermogenesis in adipocytes: Is beige the new brown? Genes \& development, 27, 234-250.

WU, R., DONG, W., CUI, X., ZHOU, M., SIMMS, H. H., RAVIKUMAR, T. S. \& WANG, P. 2007. Ghrelin down-regulates proinflammatory cytokines in sepsis through activation of the vagus nerve. Annals of surgery, 245, 480.

WU, R., DONG, W., JI, Y., ZHOU, M., MARINI, C. P., RAVIKUMAR, T. S. \& WANG, P. 2008. Orexigenic hormone ghrelin attenuates local and remote organ injury after intestinal ischemia-reperfusion. PLoS One, 3, e2026.

WU, Z., XIE, Y., BUCHER, N. \& FARMER, S. R. 1995. Conditional ectopic expression of C/EBP beta in NIH-3T3 cells induces PPAR gamma and stimulates adipogenesis. Genes \& development, 9, 2350-2363. 
XIA, Q., PANG, W., PAN, H., ZHENG, Y., KANG, J.-S. \& ZHU, S.-G. 2004. Effects of ghrelin on the proliferation and secretion of splenic $\mathrm{T}$ lymphocytes in mice. Regulatory peptides, $122,173-178$.

XUE, J.-H., YUAN, Z., WU, Y., LIU, Y., ZHAO, Y., ZHANG, W.-P., TIAN, Y.-L., LIU, W.-M., LIU, Y. \& KISHIMOTO, C. 2009. High glucose promotes intracellular lipid accumulation in vascular smooth muscle cells by impairing cholesterol influx and efflux balance. Cardiovascular research, 86, 141-150.

YANG, J., BROWN, M. S., LIANG, G., GRISHIN, N. V. \& GOLDSTEIN, J. L. 2008. Identification of the acyltransferase that octanoylates ghrelin, an appetite-stimulating peptide hormone. Cell, 132, 387-396.

YANG, J., CRADDOCK, L., HONG, S. \& LIU, Z. M. 2009. AMP-activated protein kinase suppresses LXR-dependent sterol regulatory element-binding protein-1c transcription in rat hepatoma McA-RH7777 cells. Journal of cellular biochemistry, $106,414-426$.

YAP, F., CRADDOCK, L. \& YANG, J. 2011. Mechanism of AMPK suppression of LXR-dependent Srebp-1c transcription. International journal of biological sciences, 7, 645.

YASUDA, T., MASAKI, T., KAKUMA, T. \& YOSHIMATSU, H. 2003. Centrally administered ghrelin suppresses sympathetic nerve activity in brown adipose tissue of rats. Neuroscience letters, 349, 75-78.

YOUNG, P., ARCH, J. \& ASHWELL, M. 1984. Brown adipose tissue in the parametrial fat pad of the mouse. FEBS letters, 167, 10-14.

ZHOU, G., MYERS, R., LI, Y., CHEN, Y., SHEN, X., FENYK-MELODY, J., WU, M., VENTRE, J., DOEBBER, T. \& FUJII, N. 2001. Role of AMP-activated protein kinase in mechanism of metformin action. The Journal of clinical investigation, 108, 1167-1174. 
ZHOU, Z. E., TANG, Y., JIN, X., CHEN, C., LU, Y., LIU, L. \& SHEN, C. 2016. Metformin Inhibits Advanced Glycation End Products-Induced Inflammatory Response in Murine Macrophages Partly through AMPK Activation and RAGE/NFkB Pathway Suppression. Journal of diabetes research, 2016.

ZHU, J., ZHENG, C., CHEN, J., LUO, J., SU, B., HUANG, Y., SU, W., LI, Z. \& CUI, T. 2014. Ghrelin protects human umbilical vein endothelial cells against high glucose-induced apoptosis via mTOR/P70S6K signaling pathway. Peptides, 52, 23-28.

ZOUNGAS, S., CHALMERS, J., NINOMIYA, T., LI, Q., COOPER, M., COLAGIURI, S., FULCHER, G., DE GALAN, B., HARRAP, S. \& HAMET, P. 2012. Association of HbA1c levels with vascular complications and death in patients with type 2 diabetes: evidence of glycaemic thresholds. Diabetologia, 55, 636-643. 